Supporting Information

\title{
Two-State Reactivity Mechanism of Benzene C-C Activation by Trinuclear Titanium Hydride
}

\author{
Bo Zhu, Wei Guan, ${ }^{*}$ Li-Kai Yan, and Zhong-Min Su* \\ Institute of Functional Material Chemistry \& Local United Engineering Lab for Power Battery, Faculty of \\ Chemistry, Northeast Normal University, Changchun 130024, China
}




\section{Reference 14}

Gaussian 09, Revision D.01, Frisch, M. J.; Trucks, G. W.; Schlegel, H. B.; Scuseria, G. E.; Robb, M. A.; Cheeseman, J. R.; Scalmani, G.; Barone, V.; Mennucci, B.; Petersson, G. A.; Nakatsuji, H.; Caricato, M.; Li, X.; Hratchian, H. P.; Izmaylov, A. F.; Bloino, J.; Zheng, G.; Sonnenberg, J. L.; Hada, M.; Ehara, M.; Toyota, K.; Fukuda, R.; Hasegawa, J.; Ishida, M.; Nakajima, T.; Honda, Y.; Kitao, O.; Nakai, H.; Vreven, T.; Montgomery, J. A., Jr.; Peralta, J. E.; Ogliaro, F.; Bearpark, M.; Heyd, J. J.; Brothers, E.; Kudin, K. N.; Staroverov, V. N.; Kobayashi, R.; Normand, J.; Raghavachari, K.; Rendell, A.; Burant, J. C.; Iyengar, S. S.; Tomasi, J.; Cossi, M.; Rega, N.; Millam, J. M.; Klene, M.; Knox, J. E.; Cross, J. B.; Bakken, V.; Adamo, C.; Jaramillo, J.; Gomperts, R.; Stratmann, R. E.; Yazyev, O.; Austin, A. J.; Cammi, R.; Pomelli, C.; Ochterski, J. W.; Martin, R. L.; Morokuma, K.; Zakrzewski, V. G.; Voth, G. A.; Salvador, P.; Dannenberg, J. J.; Dapprich, S.; Daniels, A. D.; Farkas, Ö.; Foresman, J. B.; Ortiz, J. V.; Cioslowski, J.; Fox, D. J. Gaussian, Inc., Wallingford CT, 2009. 


\section{Computational Details}

All geometries were optimized without symmetry constraints at the level of the (U)B3LYP hybrid functional $^{10,11}$ in the gas phase. The "guess = (fragments, N)" keyword was used to located antiferromagnetic singlet. The vibrational frequency was evaluated for each optimized structure to determine whether it is a transition state $\left(\mathrm{N}_{\mathrm{imag}}=1\right)$ or an equilibrium structure $\left(\mathrm{N}_{\mathrm{imag}}=0\right)$. Two kinds of basis sets, BSI and BSII, were employed in the calculations. In BSI, the 6-31++G(d,p) basis sets were used for the bridging hydride atoms, the 6-31G(d) basis sets were used for other main-group elements, and the Stuttgart/Dresden ECP basis sets $(\mathrm{SDD})^{\mathrm{S} 1}$ were applied for the titanium atoms. In BSII, the 6-311++G(d,p) and SDD basis sets were used for main-group and transition-metal elements, respectively. BSI was employed for the geometry optimizations, intrinsic reaction coordinate (IRC) calculations, and frequency calculations. BSII was used to evaluate the potential energy changes, where the (U)M06 method ${ }^{12}$ was employed and the solvent effect of $\mathrm{C}_{6} \mathrm{H}_{6}$ was evaluated with the conductor-like polarizable continuum model $(\mathrm{CPCM})^{13}$. All of these calculations were performed by the Gaussian 09 program package. ${ }^{14}$ Minimum energy crossing point (MECP) between two spin states was located by using the method of Morokuma et al. ${ }^{18}$

We evaluated the electronic energy $\left(E_{\text {sol }}\right)$ with zero-point energy correction in solution. For each species, the $E_{\text {sol }}$ is defined through eqn (S1):

$$
E_{\text {sol }}=E_{\text {sol }}^{\text {pot }}+E_{\text {gas }}^{v_{0}}
$$

where $E_{\text {sol }}^{\text {pot }}$ is the potential energy including non-electrostatic energy in solution and $E_{g a s}^{v_{0}}$ represents the zero-point vibrational energy in the gas phase. In a bimolecular process, such as the coordination of benzene with the trinuclear titanium polyhydride and $\mathrm{H}_{2}$ dissociation, the entropy change must be taken into consideration because the entropy considerably decreases in the coordination of benzene and considerably increases in $\mathrm{H}_{2}$ dissociation. In this case, Gibbs energy $\left(G_{\text {sol }}^{o}\right)$ must be evaluated as follows:

$$
\begin{aligned}
G_{\text {sol }}^{o} & =H_{0}-T\left(S_{r}^{o}+S_{v}^{o}+S_{t}^{o}\right) \\
& =E^{T}+P \Delta V-T\left(S_{r}^{o}+S_{v}^{o}+S_{t}^{o}\right) \\
& =E_{\text {sol }}+E_{\text {therm }}-T\left(S_{r}^{o}+S_{v}^{o}+S_{t}^{o}\right)
\end{aligned}
$$

where $\Delta V$ is 0 in solution, $E_{\text {therm }}$ is the thermal correction by translational, vibrational, and rotational movements, and $S_{r}^{o}, S_{v}^{o}$, and $S_{t}^{o}$ are rotational, vibrational, and translational entropies, respectively. In general, the Sackur-Tetrode equation is used to evaluate translational entropy $S_{t}^{o}$. In solution, however, the usual Sackur-Tetrode equation cannot be directly applied to the evaluation of $S_{t}^{o}$, because the 
translation movement is suppressed very much in solution. ${ }^{\mathrm{S} 2}$ In this context, the translational entropy was corrected with the method developed by Whitesides et al., ${ }^{\mathrm{S} 3}$ where the rotational entropy was evaluated in a normal manner. Thermal correction and entropy contributions of vibration movements to the Gibbs energy were evaluated with the frequencies calculated at $298.15 \mathrm{~K}$ and $1 \mathrm{~atm}$.

\section{References}

(S1) Dolg, M.; Wedig, U.; Stoll, H.; Preuss, H. J. Chem. Phys. 1987, 86, 866.

(S2) (a) Sakaki, S.; Ohnishi, Y.; Sato, H. The Chemical Record 2010, 10, 29. (b) Ishikawa, A.; Nakao, Y.;

Sato, H.; Sakaki, S. Dalton Trans. 2010, 39, 3279. (c) Ishikawa, A.; Nakao, Y.; Sato, H.; Sakaki, S. Inorg.

Chem. 2009, 48, 8154. (d) Ohnishi, Y.; Nakao, Y.; Sato, H.; Hiyama, T.; Sakaki, S. Organometallics 2009, $28,2583$.

(S3) Mammen, M.; Shakhnovich, E. I.; Deutch, J. M.; Whitesides, G. M. J. Org. Chem. 1998, 63, 3821. 


\section{Evaluation of Computational Methods}

Table S1 Selected important geometrical parameters for trinuclear titanium hydride complexes $\mathbf{1}$ and $\mathbf{2}$ optimized at the (U)B3LYP/BSI level (bond lengths and angles in angstroms and degrees, respectively).

\begin{tabular}{|c|c|c|c|c|c|c|c|c|c|c|}
\hline \multirow[t]{2}{*}{ system } & \multicolumn{7}{|c|}{1} & \multicolumn{3}{|c|}{2} \\
\hline & $\exp ^{a}$ & $1^{\mathrm{AF}}$ & $|\Delta|^{b}$ & $1^{\mathrm{CS}}$ & $|\Delta|$ & $1^{T}$ & $|\Delta|$ & $\exp ^{a}$ & $2^{\mathrm{CS}}$ & $|\Delta|$ \\
\hline Ti1-Ti2 & 2.65 & 2.68 & 0.03 & 2.57 & 0.08 & 2.73 & 0.08 & 2.84 & 2.76 & 0.08 \\
\hline Ti1-Ti3 & 2.65 & 2.69 & 0.04 & 2.58 & 0.07 & 2.74 & 0.09 & 2.68 & 2.64 & 0.04 \\
\hline Ti1-Ti3 & 2.65 & 2.69 & 0.04 & 2.57 & 0.1 & 2.74 & 0.09 & 2.68 & 2.63 & 0.05 \\
\hline Ti1-Ti2-Ti3 & 60.0 & 60.1 & 0.1 & 59.9 & 0.1 & 59.8 & 0.2 & 58.1 & 58.4 & 0.3 \\
\hline Ti1-Ti3-Ti2 & 60.1 & 59.8 & 0.3 & 60.0 & 0.1 & 60.0 & 0.1 & 63.8 & 63.2 & 0.6 \\
\hline Ti3-Ti1-Ti2 & 60.0 & 59.8 & 0.2 & 60.1 & 0.1 & 60.2 & 0.2 & 58.2 & 58.4 & 0.2 \\
\hline Ti1-Cp'1 ${ }^{c}$ & 2.36 & 2.37 & 0.01 & 2.38 & 0.02 & 2.37 & 0.01 & 2.40 & 2.41 & 0.01 \\
\hline Ti2-Cp' $2^{c}$ & 2.36 & 2.38 & 0.02 & 2.38 & 0.02 & 2.37 & 0.01 & 2.40 & 2.41 & 0.01 \\
\hline Ti3-Cp'3 ${ }^{c}$ & 2.36 & 2.36 & 0.00 & 2.38 & 0.02 & 2.36 & 0.00 & 2.39 & 2.41 & 0.02 \\
\hline
\end{tabular}

${ }^{a}$ Reference 9. ${ }^{b}$ Absolute difference between the computed data and experimental measurements. ${ }^{c}$ The average distance between $\mathrm{Ti}$ and $\mathrm{C}$ atoms of $\mathrm{Cp}^{\prime}$ ring.
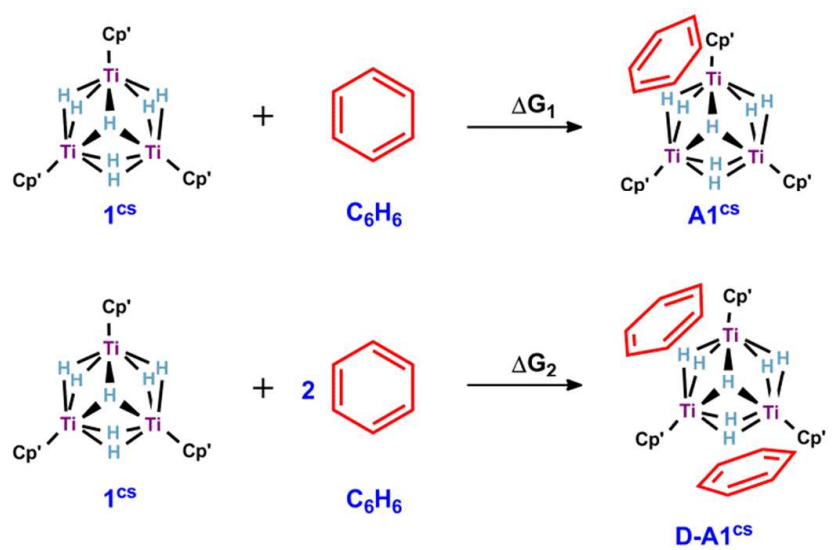

Scheme S1 Reactions of $1^{\mathrm{CS}}$ and benzene molecules to evaluate the effect of the dispersion between them.

Table S2 Reaction energies (in kcal/mol) of $\mathbf{1}^{\mathrm{CS}}$ to $\mathbf{A 1}{ }^{\mathrm{CS}}$ and $\mathbf{D}-\mathbf{A 1}{ }^{\mathrm{CS}}$ shown in Scheme $\mathrm{S} 1$ with various methods.

\begin{tabular}{lcc}
\hline \multicolumn{1}{c}{ method } & $\Delta \mathrm{G}_{1}$ & $\Delta \mathrm{G}_{2}$ \\
\hline B3LYP(CPCM)/BSII/B3LYP/BSI & 6.4 & -2.1 \\
M06(CPCM)/BSII//B3LYP/BSI & -5.3 & -12.4 \\
B3LYP-D3(CPCM)/BSII/B3LYP/BSI & -5.2 & -12.3 \\
B3LYP-D3(CPCM)/BSII/B3LYP-D3/BSI & -4.0 & -8.5 \\
\hline
\end{tabular}


Table S3 Calculated relative Gibbs free energy (in $\mathrm{kcal} / \mathrm{mol}$ ) with different electronic energies evaluated by the (U)M06 and (U)B3LYP-D3 methods for the transition states $\mathbf{T S 5}^{\mathbf{A F}}, \mathbf{T S 5}^{\mathbf{T}}$, and $\mathbf{T S 5}^{\mathbf{C S}}$, respectively.

\begin{tabular}{lccc}
\hline \multicolumn{1}{c}{ method } & TS5 $^{\mathbf{A F}}$ & TS5 $^{\mathbf{T}}$ & TS5 $^{\mathbf{C S}}$ \\
\hline (U)M06(CPCM)/BSII//(U)B3LYP/BSI & 0.0 & 2.7 & 8.9 \\
(U)B3LYP-D3(CPCM)/BSII//(U)B3LYP/BSI & 0.0 & 2.2 & 16.6 \\
\hline
\end{tabular}

Table S4 The relative Gibbs free energy values (in $\mathrm{kcal} / \mathrm{mol}$ ) of some intermediates and transition states calculated at the (U)M06(CPCM)/BSII//(U)B3LYP/BSI level.

\begin{tabular}{lccccc}
\hline & $\mathbf{1}^{\mathbf{A F}}$ & $\mathbf{T S 4}^{\mathbf{T}}$ & $\mathbf{A 5}^{\mathbf{T}}$ & $\mathbf{T S 4}^{\mathbf{A F}}$ & $\mathbf{A 5}^{\mathbf{A F}}$ \\
\hline$\Delta \mathrm{G}^{\circ}$ (with $S_{t}^{o}$ correction) & 0.0 & 7.7 & 11.3 & 11.1 & 14.3 \\
$\Delta \mathrm{G}^{\circ}$ (without $S_{t}^{o}$ correction) & 0.0 & 13.8 & 11.3 & 17.1 & 14.3 \\
\hline
\end{tabular}

Table S5 The relative Gibbs free energy values (in $\mathrm{kcal} / \mathrm{mol}$ ) of some intermediates and transition states calculated at the (U)B3LYP/BSI level and the (U)M06(CPCM)/BSII//(U)B3LYP/BSI level.

\begin{tabular}{lccccc}
\hline & $\mathbf{1}^{\mathbf{A F}}$ & $\mathbf{A 6}^{\mathbf{A F}}$ & $\mathbf{T S 6}^{\mathbf{A F}}$ & $\mathbf{B 7}^{\mathbf{C S}}$ & TS15 $^{\mathbf{C S}}$ \\
\hline$\Delta \mathrm{G}^{\circ}$ (without single point energy correction) & 0.0 & 20.7 & 23.1 & 23.1 & 23.4 \\
$\Delta \mathrm{G}^{\circ}$ (with single point energy correction) & 0.0 & 8.0 & 7.1 & 3.0 & 1.6 \\
\hline
\end{tabular}

\section{Binding Energies of the $\mu-\mathrm{H}$ and $\mu_{3}-\mathrm{H}$}

The binding energies of the $\mu-\mathrm{H}$ and $\mu_{3}-\mathrm{H}$ with the rest of $\mathbf{1}^{\mathrm{CS}}$ were calculated at the B3LYP/BSII level as the following formula.

$$
E_{\mathrm{b}}=E_{1} c s-E_{1} c s_{-\mathrm{H}}-E_{\mathrm{H}}-E_{\mathrm{BSSE}}
$$

where $E_{\mathrm{b}}$ is the binding energies, $E_{\mathbf{1}}{ }^{c s}$ is defined as the energy of complex $\mathbf{1}^{\mathrm{CS}}\left(\left(\mathbf{C p}{ }^{\prime} \mathbf{T i}\right)_{3}\left(\boldsymbol{\mu}_{\mathbf{3}}-\mathbf{H}\right)(\boldsymbol{\mu}-\mathbf{H})_{6}\right)$, $E_{1}{ }^{c s}{ }_{-\mathbf{H}}$ is the energy of the $\left(\mathbf{C p}^{\prime} \mathbf{T i}\right)_{3}\left(\boldsymbol{\mu}_{3}-\mathbf{H}\right)(\boldsymbol{\mu}-\mathbf{H})_{5}$ moiety or $\left(\mathbf{C p}^{\prime} \mathbf{T i}\right)_{3}(\boldsymbol{\mu}-\mathbf{H})_{6}$ moiety in $\mathbf{1}^{\mathrm{Cs}}, E_{\mathbf{H}}$ denotes the energy of the corresponding $\mu-\mathrm{H}$ or $\mu_{3}-\mathrm{H}$ in $\mathbf{1}^{\mathrm{CS}}$, and $E_{\mathrm{BSSE}}$ is the energy of basis set superposition error, respectively. A similar methodology $\left(E_{\mathrm{b}}=E_{\mathbf{1}^{T}}-E_{\left(\mathbf{1}^{\mathbf{T}}\right)-\mathbf{H}}-E_{\mathbf{H}}-E_{\mathrm{BSSE}}\right)$ was employed to $\mathbf{1}^{\mathbf{T}}$ discussed in the text.

Table S6 Binding energies $\left(E_{\mathrm{b}}\right.$, in kcal/mol) of the $\mu-\mathrm{H}$ and $\mu_{3}-\mathrm{H}$ with the rest of $\mathbf{1}^{\mathrm{CS}}$ and $\mathbf{1}^{\mathbf{T}}$, respectively.

\begin{tabular}{lcc}
\hline & $\mathbf{1}^{\mathbf{C S}}$ & $\mathbf{1}^{\mathbf{T}}$ \\
\hline$E_{\mathrm{b}}(\mu-\mathrm{H})$ & -189.1 & -187.9 \\
$E_{\mathrm{b}}\left(\mu_{3}-\mathrm{H}\right)$ & -180.6 & -190.4 \\
\hline
\end{tabular}




\section{Electronic Structure Calculations of $1^{\mathrm{AF}}, \mathrm{A6}{ }^{\mathrm{CS}}, \mathrm{A} 7^{\mathrm{AF}}$ and $\mathrm{A} 7^{\mathrm{T}}$.}

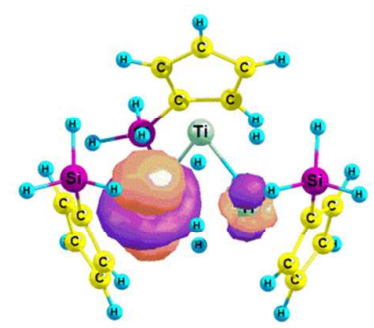

1.18

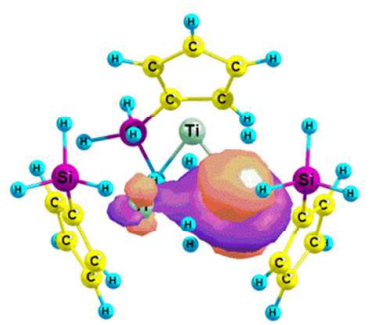

0.82

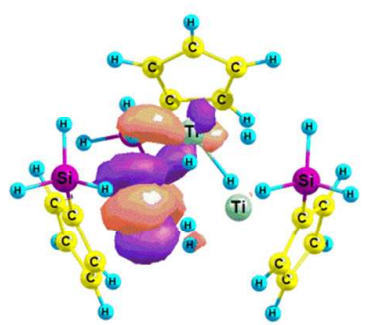

0.01

$\varphi_{1}$

$\varphi_{3}$

Figure S1. The natural orbital and occupation number of the lowest singlet state calculated by CASPT2/CASSCF on the geometry of $\mathbf{1}^{\mathrm{AF}}$.

Because three Ti atoms in complex $\mathbf{1}$ are bridged by hydride, we considered the seven s-orbitals of hydrides and three 3d-orbitals of Ti atoms in CASPT2/CASSCF calculations. Thus, the active space includes 14 electrons and 9 MOs in total, which is referred as CASSCF (16e, 10o). In the converged CASSCF wave-function, three 3p-orbitals of Ti move into the active space. However, all the s-orbital of hydride and 3p-orbital of Ti are all nearly doubly occupied, indicating that they contribute little to the configuration interaction. On the other hand, three MOs composed of 3d-orbitals of $\mathrm{Ti}$ are the most important; see $\varphi_{1}, \varphi_{2}$, and $\varphi_{3}$ and their occupation numbers in Figure $\mathrm{S} 1$. The lowest singlet state has an open-shell electronic structure, in which $\varphi_{1}^{\alpha} \varphi_{2}^{\beta} \varphi_{3}^{0}$ is the dominant configuration with weight of $77 \%$ and $\varphi_{1}^{2} \varphi_{2}^{0} \varphi_{3}^{0}$ is the second configuration with weight of $14 \%$. The CASPT2/CASSCF (2e, 3o) calculation, which only considers these three MOs, gives almost the same results to CASSCF (16e, 10o) calculation, where the dominant configuration $\varphi_{1}^{\alpha} \varphi_{2}^{\beta} \varphi_{3}^{0}$ has weight of $75 \%$ and the second configuration has weight of $12 \%$. The lowest triplet state, whose dominant configuration $\varphi_{1}^{\alpha} \varphi_{2}^{\alpha} \varphi_{3}^{0}$ has weight of $99 \%$, is about 4.2 $\mathrm{kcal} / \mathrm{mol}$ higher than the ground state. CASPT2/CASSCF calculations show that complex $\mathbf{1}$ has an open-shell singlet ground state. Because $\varphi_{1}$ and $\varphi_{2}$ are mainly localized on Ti1 and Ti2, respectively, complex 1 has a configuration of Ti1(III)Ti2(III)Ti3(IV) in formal sense.

To further confirm the reliabilities of the method and the relative stability of intermediates $\mathbf{A 6} \mathbf{6}^{\mathbf{C S}}, \mathbf{A} 7^{\mathbf{A F}}$ and $\mathbf{A} 7^{\mathbf{T}}$, the energies of different spin states of them have been investigated by CASPT2/CASSCF calculations. Because the geometry of benzene distorted significantly, the three $\pi$ MOs and three $\pi^{*}$ MOs are included in the active space. In addition, three MOs with 4 electrons of $\mathrm{Ti}_{3}$ moiety are considered in the active space. In total, the active space includes 10 electrons and 9 MOs (see Figure S2). The CASSCF 
calculations indicate that three Ti atoms of intermediates $\mathbf{A 6}{ }^{\mathbf{C S}}, \mathbf{A} 7^{\mathbf{A F}}$ and $\mathbf{A} 7^{\mathbf{T}}$ have a configuration of Ti1(III)Ti2(III)Ti3(II) in formal sense. The doubly occupied d-orbital of Ti3 interacts with one $\pi^{*}$ MOs forming bonding $\mathrm{MO} \varphi_{8}$ and anti-bonding $\operatorname{MO} \varphi_{5}$. Two d-orbitals of Ti1 and Ti2 form a bonding $\operatorname{MO} \varphi_{7}$ and an anti-bonding $\mathrm{MO} \varphi_{9}$. Because of the long distance between Ti2 and Ti3, the energy gap between $\varphi_{7}$ and $\varphi_{9}$ are expected to be quite small. Thus, the electron configuration with electron excitation from $\varphi_{7}$ to $\varphi_{9}$ contributes significantly to the ground state (see Tables S7-S8). Note from Table S8, it can be found that the dominant configurations of $\mathbf{A} 7^{\mathbf{A F}}$ (singlet) are $\varphi_{1}^{2} \varphi_{2}^{2} \varphi_{3}^{2} \varphi_{4}^{0} \varphi_{5}^{0} \varphi_{6}^{0} \varphi_{7}^{2} \varphi_{8}^{2} \varphi_{9}^{0}$ with weight of $41 \%$ and $\varphi_{1}^{2} \varphi_{2}^{2} \varphi_{3}^{2} \varphi_{4}^{0} \varphi_{5}^{0} \varphi_{6}^{0} \varphi_{7}^{0} \varphi_{8}^{2} \varphi_{9}^{2}$ with weight of $27 \%$, respectively. It indicates that there is strong interaction between the two important configurations. Furthermore, the occupation numbers in $\varphi_{7}$ and $\varphi_{9}$ of $\mathbf{A} 7^{\mathbf{A F}}$ (singlet) are 1.14 and 0.85 , respectively, indicating that they are singly occupied (Table S7) and thus antiferromagnetic coupling.

All the CASPT2/CASSCF calculations are carried out on the geometry optimized by DFT. The LANL2DZ ECP basis set is used for Ti atoms, SDD ECP basis set for Si atoms, cc-pVDZ basis set for C atoms and bridging-H atoms, and cc-pVDZ basis set without $\mathrm{p}$-functions for hydrogen atoms bond with $\mathrm{Si} / \mathrm{C}$ atom.

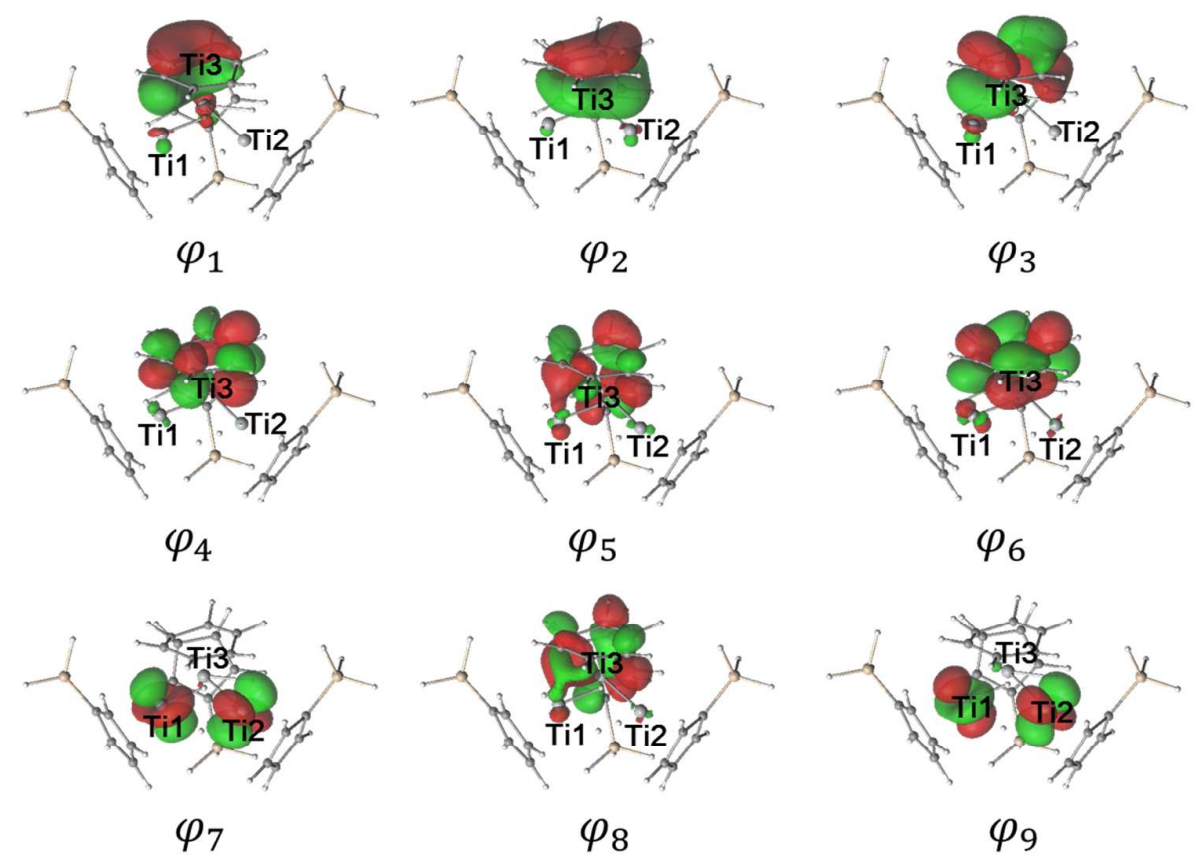

Figure S2. The active space for CASSCF calculations of $\mathbf{A 6}{ }^{\mathbf{C S}}, \mathbf{A} 7^{\mathbf{A F}}$ and $\mathbf{A} 7^{\mathbf{T}}$. 
Table S7 The relative energy (in $\mathrm{kcal} / \mathrm{mol}$ ) and occupation number. ${ }^{a}$

\begin{tabular}{ccccccccc}
\hline & \multicolumn{2}{c}{$\mathbf{A 6}^{\mathbf{C S}}$} & & \multicolumn{2}{c}{$\mathbf{A 7 ^ { \mathbf { A F } }}$} & & \multicolumn{2}{c}{$\mathbf{A 7}^{\mathbf{T}}$} \\
\cline { 2 - 3 } \cline { 7 - 8 } \cline { 7 - 8 } & singlet & triplet & & singlet & triplet & & singlet & triplet \\
\hline$\Delta \mathrm{E}$ & 0.0 & 43.0 & & 29.6 & 32.8 & & 31.8 & 33.8 \\
$\varphi_{1}$ & 1.979 & 1.979 & & 1.981 & 1.981 & & 1.983 & 1.982 \\
$\varphi_{2}$ & 1.975 & 1.973 & & 1.956 & 1.957 & & 1.954 & 1.956 \\
$\varphi_{3}$ & 1.934 & 1.935 & & 1.931 & 1.931 & & 1.928 & 1.929 \\
$\varphi_{4}$ & 0.037 & 0.041 & & 0.049 & 0.048 & & 0.050 & 0.050 \\
$\varphi_{5}$ & 0.252 & 0.326 & & 0.275 & 0.276 & & 0.273 & 0.275 \\
$\varphi_{6}$ & 0.074 & 0.065 & & 0.077 & 0.075 & & 0.078 & 0.076 \\
$\varphi_{7}$ & 1.727 & 1.050 & & 1.149 & 1.001 & & 1.115 & 1.000 \\
$\varphi_{8}$ & 1.639 & 1.678 & & 1.733 & 1.731 & & 1.735 & 1.733 \\
$\varphi_{9}$ & 0.383 & 0.952 & & 0.859 & 0.999 & & 0.883 & 0.999 \\
\hline
\end{tabular}

${ }^{a}$ Single point CASPT2/CASSCF calculation on the DFT-optimized geometries.

Table S8 Main electronic configurations, natural orbitals ( $\psi$ i) and the weight of contributions for the intermediates $\mathbf{A 6}{ }^{\mathbf{C S}}, \mathbf{A} 7^{\mathbf{A F}}$ and $\mathbf{A} 7^{\mathbf{T}}$ at singlet and triplet states.

\begin{tabular}{ll}
\hline \multicolumn{1}{c}{ state } & \multicolumn{1}{c}{ main configuration and weight } \\
\hline $\mathbf{A} 6^{\mathbf{C S}}$ (singlet) & $\varphi_{1}^{2} \varphi_{2}^{2} \varphi_{3}^{2} \varphi_{4}^{0} \varphi_{5}^{0} \varphi_{6}^{0} \varphi_{7}^{2} \varphi_{8}^{2} \varphi_{9}^{0}(60 \%)$ \\
$\mathbf{A 6}^{\mathbf{C S}}$ (triplet) & $\varphi_{1}^{2} \varphi_{2}^{2} \varphi_{3}^{2} \varphi_{4}^{0} \varphi_{5}^{0} \varphi_{6}^{0} \varphi_{7}^{1} \varphi_{8}^{2} \varphi_{9}^{1}(62 \%)$ \\
$\mathbf{A} 7^{\mathbf{A F}}$ (singlet) & $\varphi_{1}^{2} \varphi_{2}^{2} \varphi_{3}^{2} \varphi_{4}^{0} \varphi_{5}^{0} \varphi_{6}^{0} \varphi_{7}^{2} \varphi_{8}^{2} \varphi_{9}^{0}(41 \%)+\varphi_{1}^{2} \varphi_{2}^{2} \varphi_{3}^{2} \varphi_{4}^{0} \varphi_{5}^{0} \varphi_{6}^{0} \varphi_{7}^{0} \varphi_{8}^{2} \varphi_{9}^{2} \quad(27 \%)$ \\
$\mathbf{A} 7^{\mathbf{A F}}$ (triplet) & $\varphi_{1}^{2} \varphi_{2}^{2} \varphi_{3}^{2} \varphi_{4}^{0} \varphi_{5}^{0} \varphi_{6}^{0} \varphi_{7}^{1} \varphi_{8}^{2} \varphi_{9}^{1}(78 \%)$ \\
$\mathbf{A} 7^{\mathbf{T}}$ (singlet) & $\varphi_{1}^{2} \varphi_{2}^{2} \varphi_{3}^{2} \varphi_{4}^{0} \varphi_{5}^{0} \varphi_{6}^{0} \varphi_{7}^{2} \varphi_{8}^{2} \varphi_{9}^{0}(36 \%)+\varphi_{1}^{2} \varphi_{2}^{2} \varphi_{3}^{2} \varphi_{4}^{0} \varphi_{5}^{0} \varphi_{6}^{0} \varphi_{7}^{0} \varphi_{8}^{2} \varphi_{9}^{2} \quad(26 \%)$ \\
$\mathbf{A} 7^{\mathbf{T}}$ (triplet) & $\varphi_{1}^{2} \varphi_{2}^{2} \varphi_{3}^{2} \varphi_{4}^{0} \varphi_{5}^{0} \varphi_{6}^{0} \varphi_{7}^{1} \varphi_{8}^{2} \varphi_{9}^{1}(60 \%)$ \\
\hline
\end{tabular}




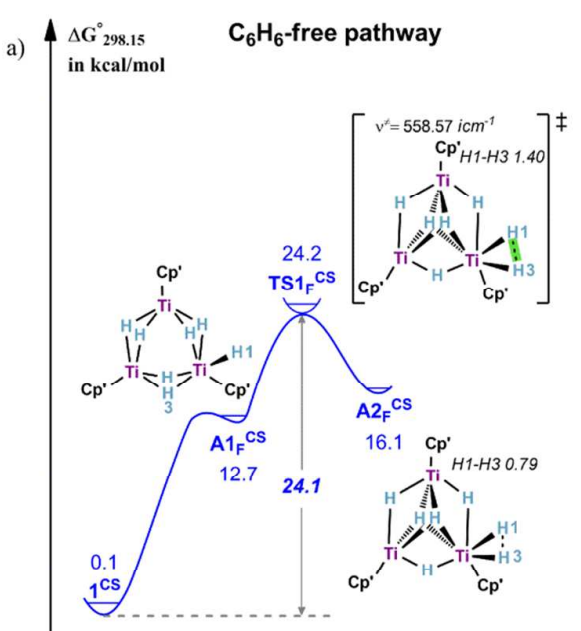

M06(СРCM)/BSII/B3LYP/BSI level

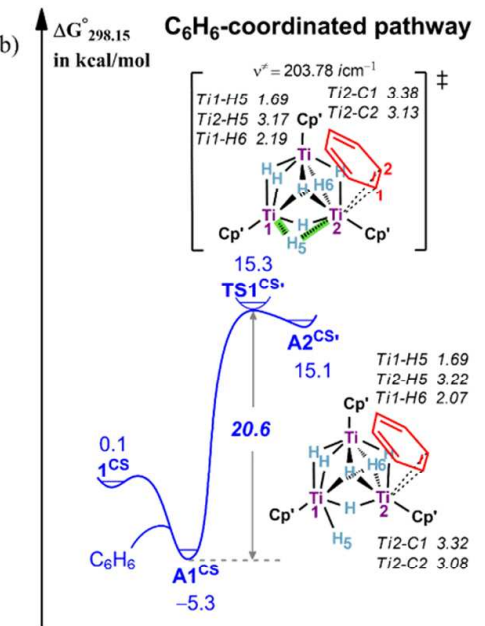

M06(СРСM)/BSII//B3LYP/BSI level

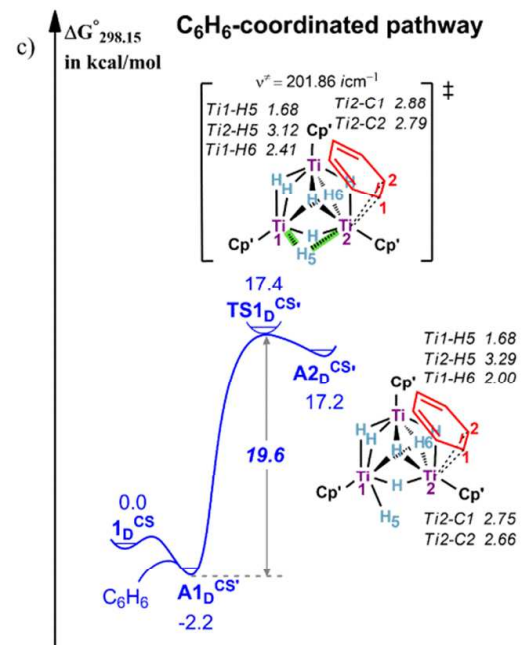

M06(CPCM)/BSII//B3LYP-D3/BSI level
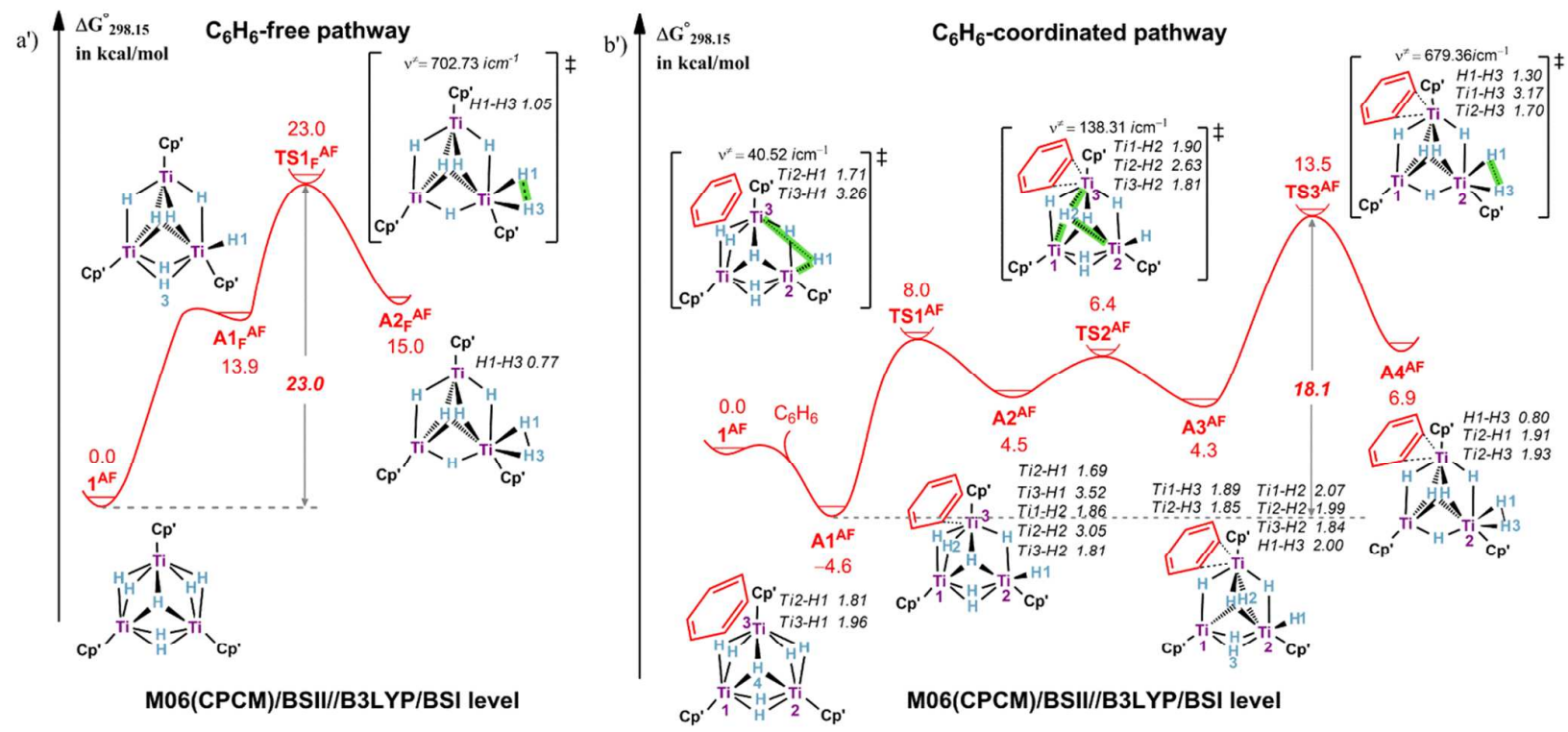

Figure S3. Comparisons of $\mathbf{C}_{6} \mathbf{H}_{6}$-free and $\mathbf{C}_{6} \mathbf{H}_{6}$-coordinated mechanisms with closed-shell singlet (a-c) and antiferromagnetic singlet (a'-b') states, respectively. 


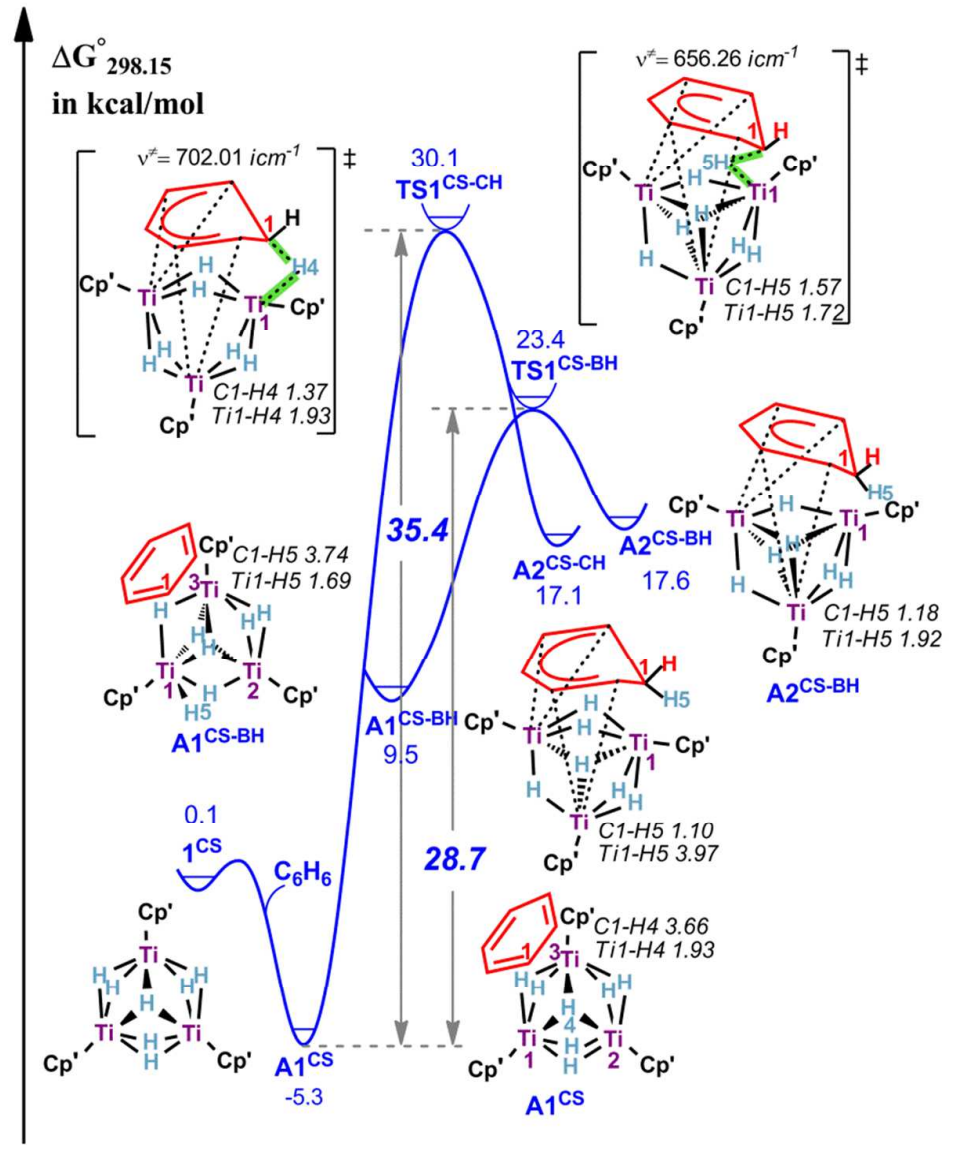

Figure S4. Gibbs energy profiles $\left(\Delta \mathrm{G}_{298.15}^{\mathrm{o}}\right)$ of the inter-molecular hydride transfer from $\mathbf{1}^{\mathrm{CS}}$ to $\mathbf{C}_{\mathbf{6}} \mathbf{H}_{\mathbf{6}}$ prior to the $\mathrm{H}-\mathrm{H}$ bond formation in the $\mathbf{C}_{6} \mathbf{H}_{6}$-coordinated mechanism. 


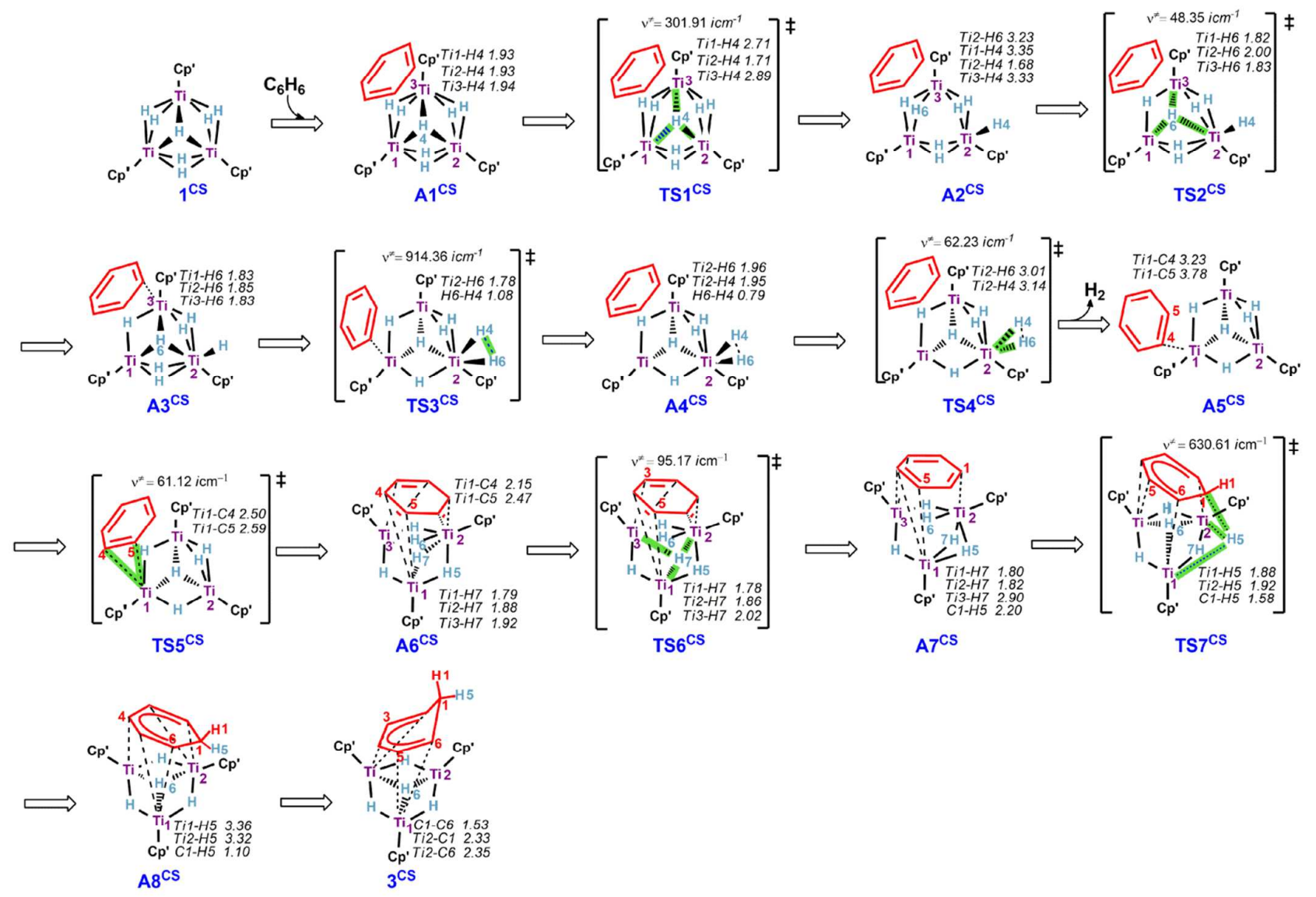

Figure S5. Geometry structures (bond distances in $\AA$ ) of stationary points in the closed-shell singlet surface of Figure 1. 

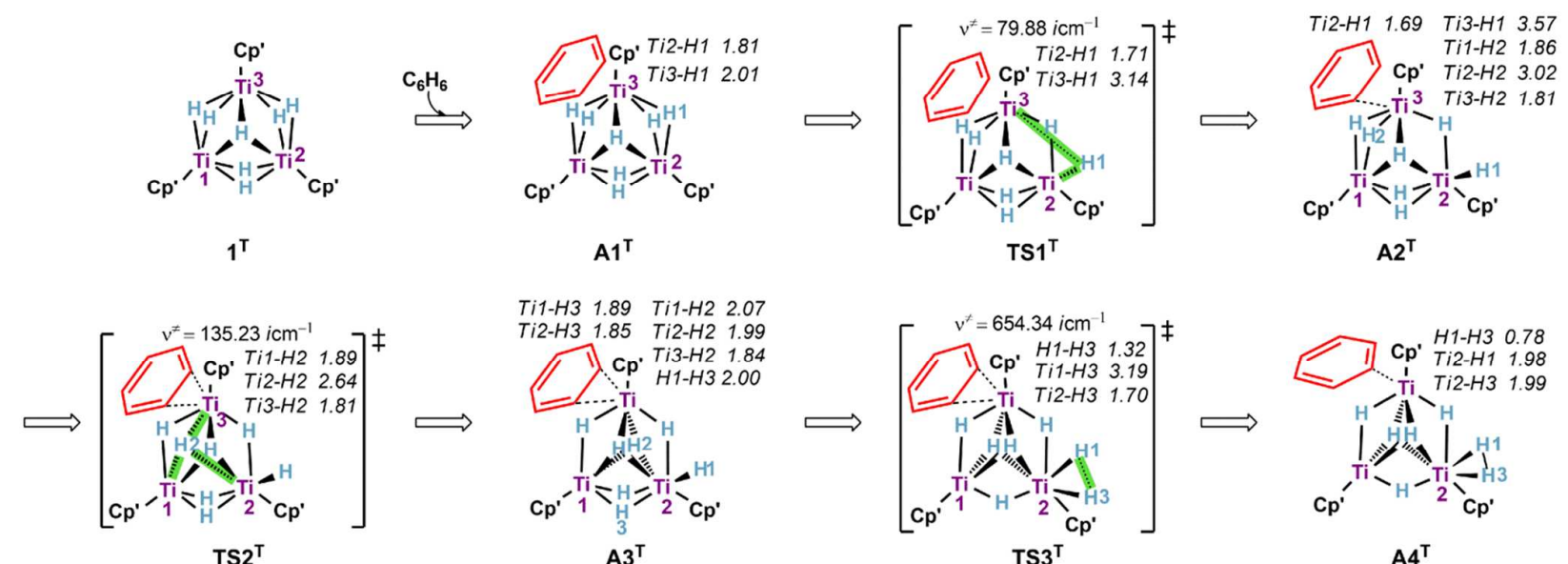

$A 2^{\top}$
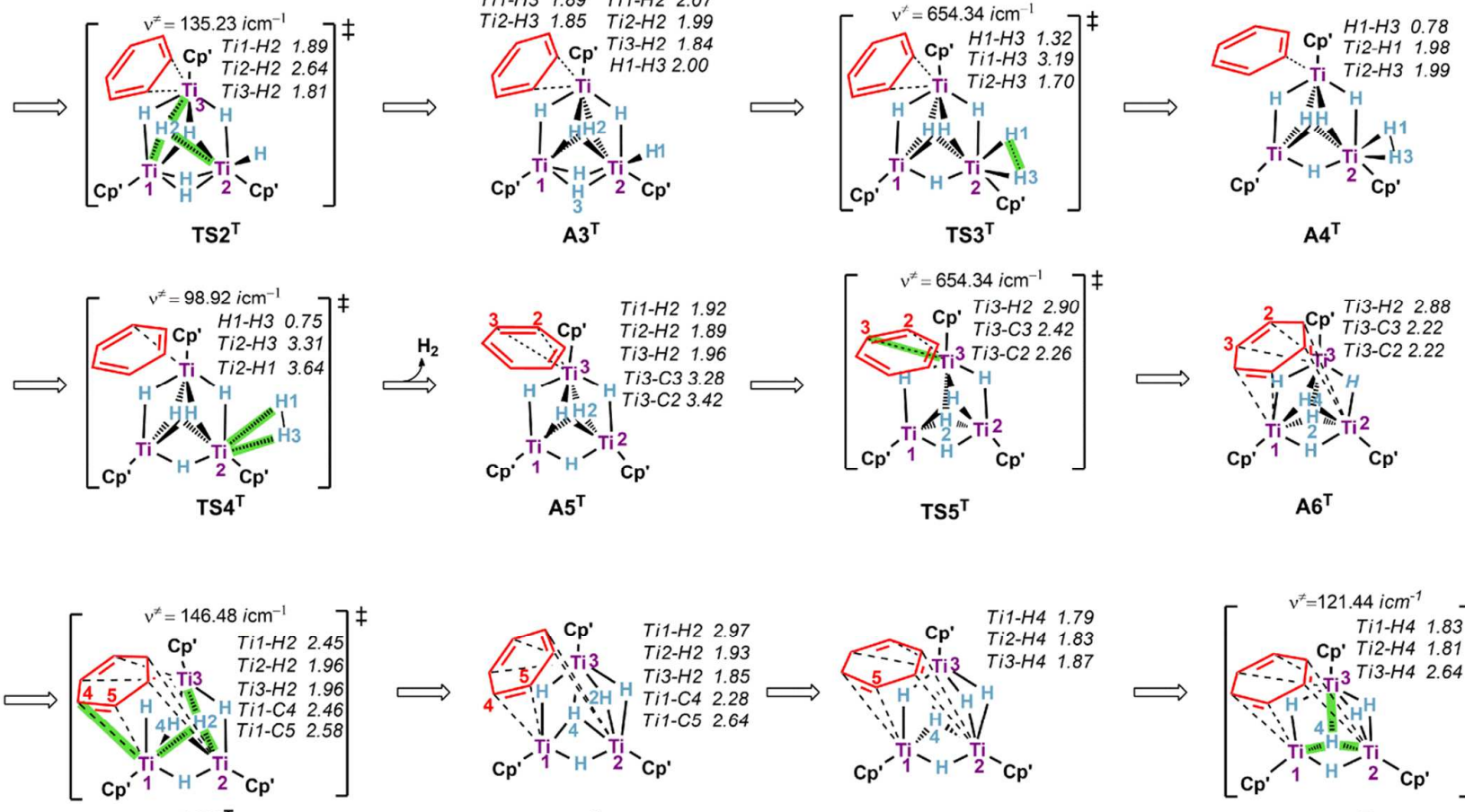

TS6 $^{\top}$
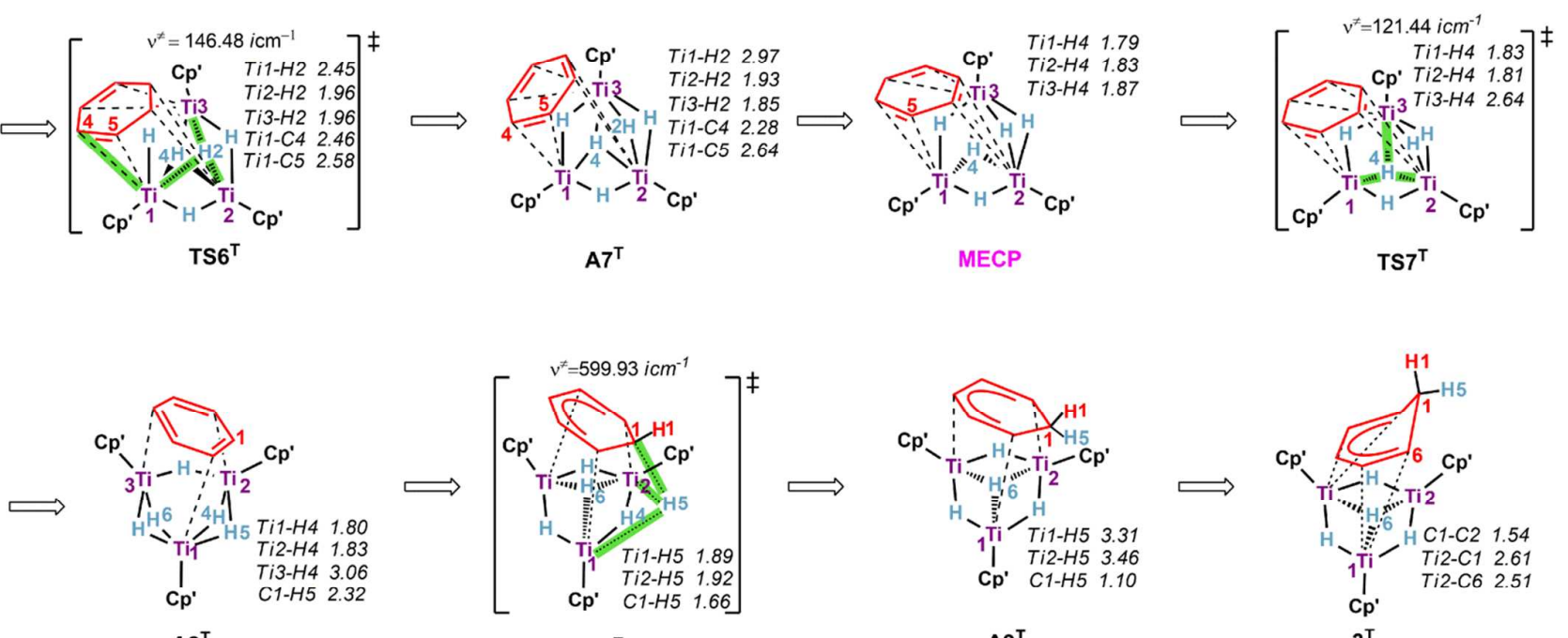

$\mathbf{T S}^{\top}$
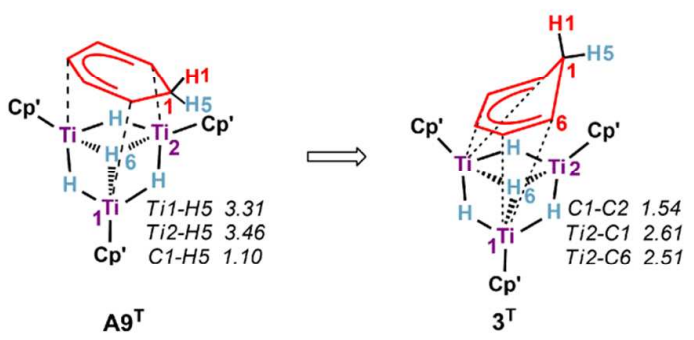

Figure S6. Geometry structures (bond distances in $\AA$ ) of stationary points in the triplet surface of Figure 1 . 


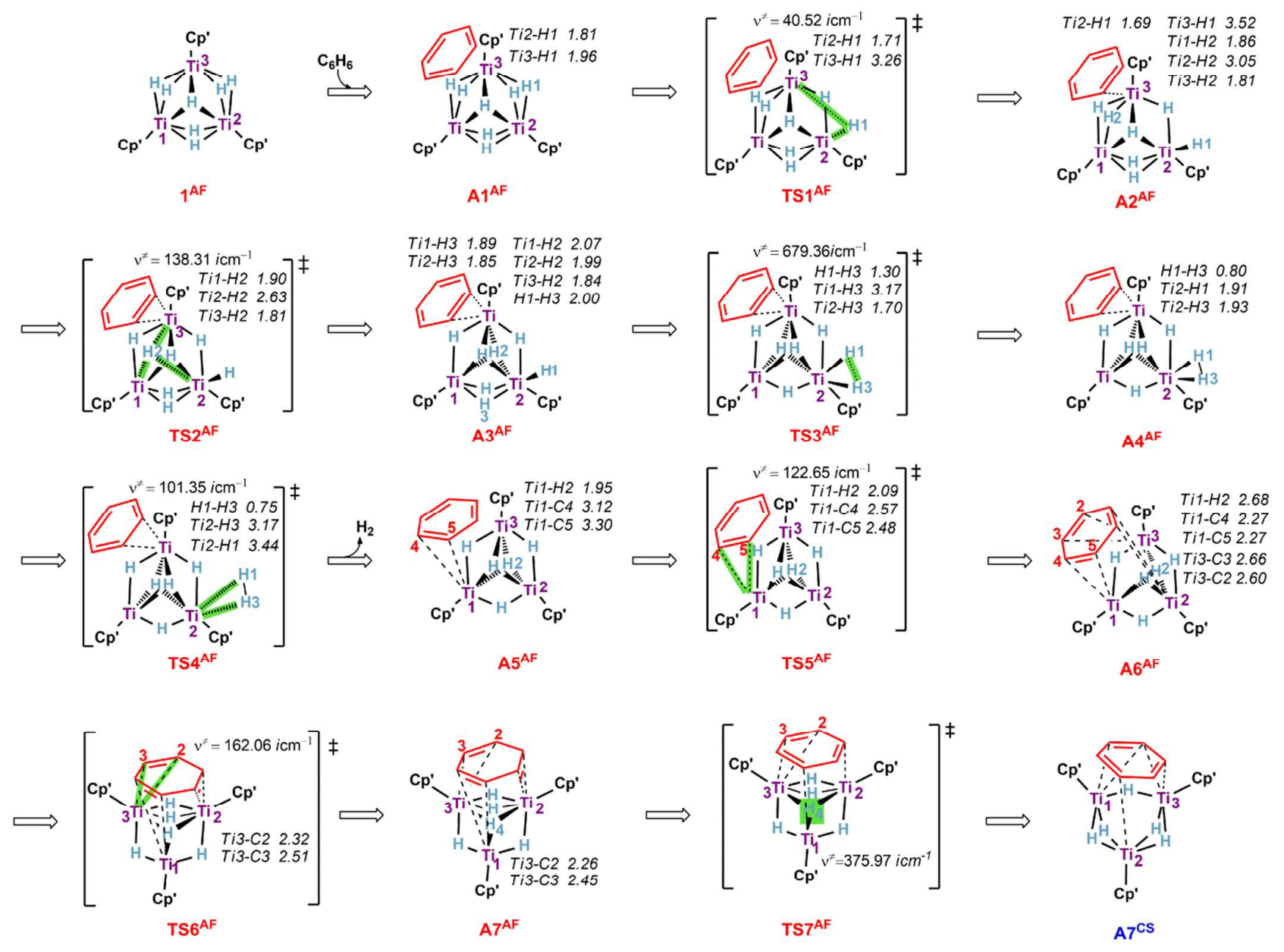

Figure S7. Geometry structures (bond distances in $\AA$ ) of stationary points in the antiferromagnetic singlet surface of Figure 1. 


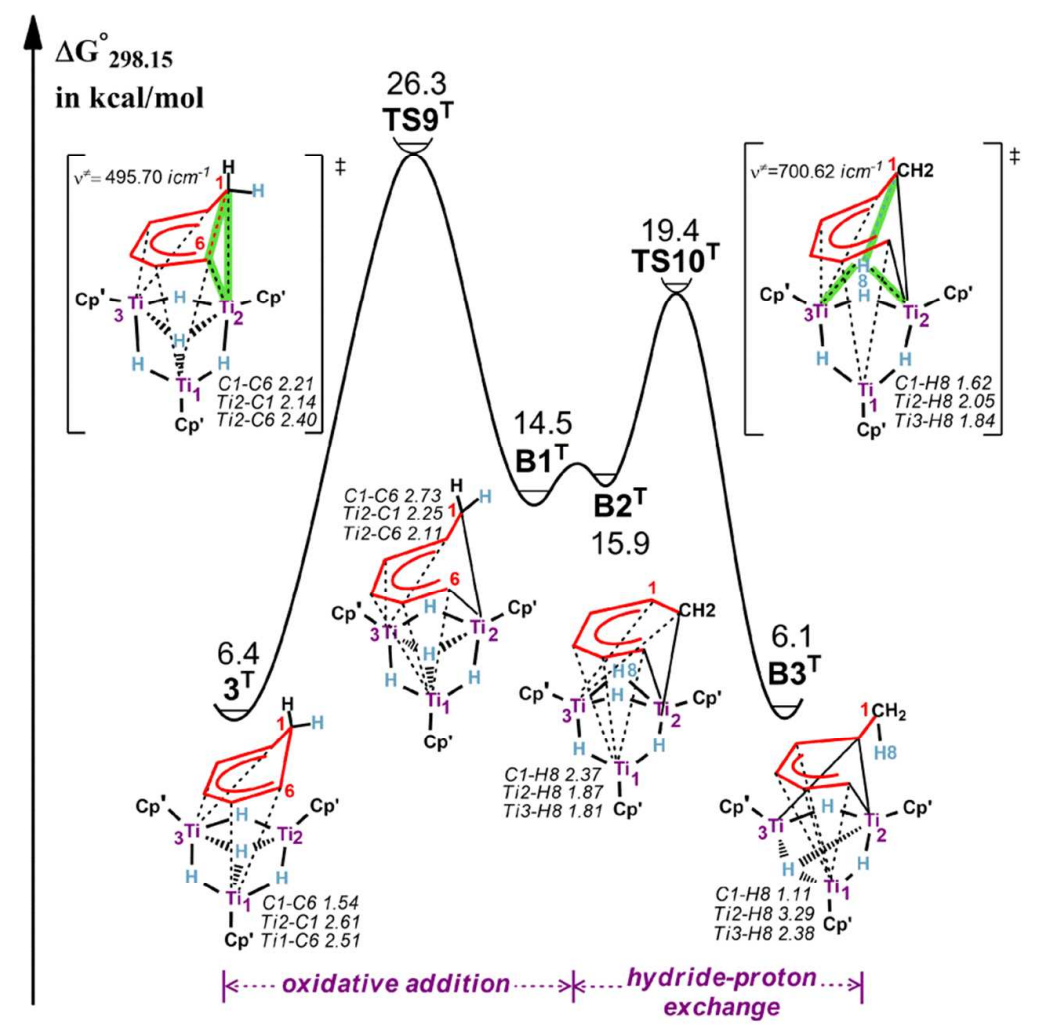

Figure S8. Geometry structures (bond distances in $\AA$ ) and the triplet Gibbs energy profile $\left(\Delta \mathrm{G}_{298.15}^{\circ}\right)$ of $\mathbf{3}^{\mathbf{T}}$ $\rightarrow \mathbf{B 3}^{\mathbf{T}}$ transformation. 


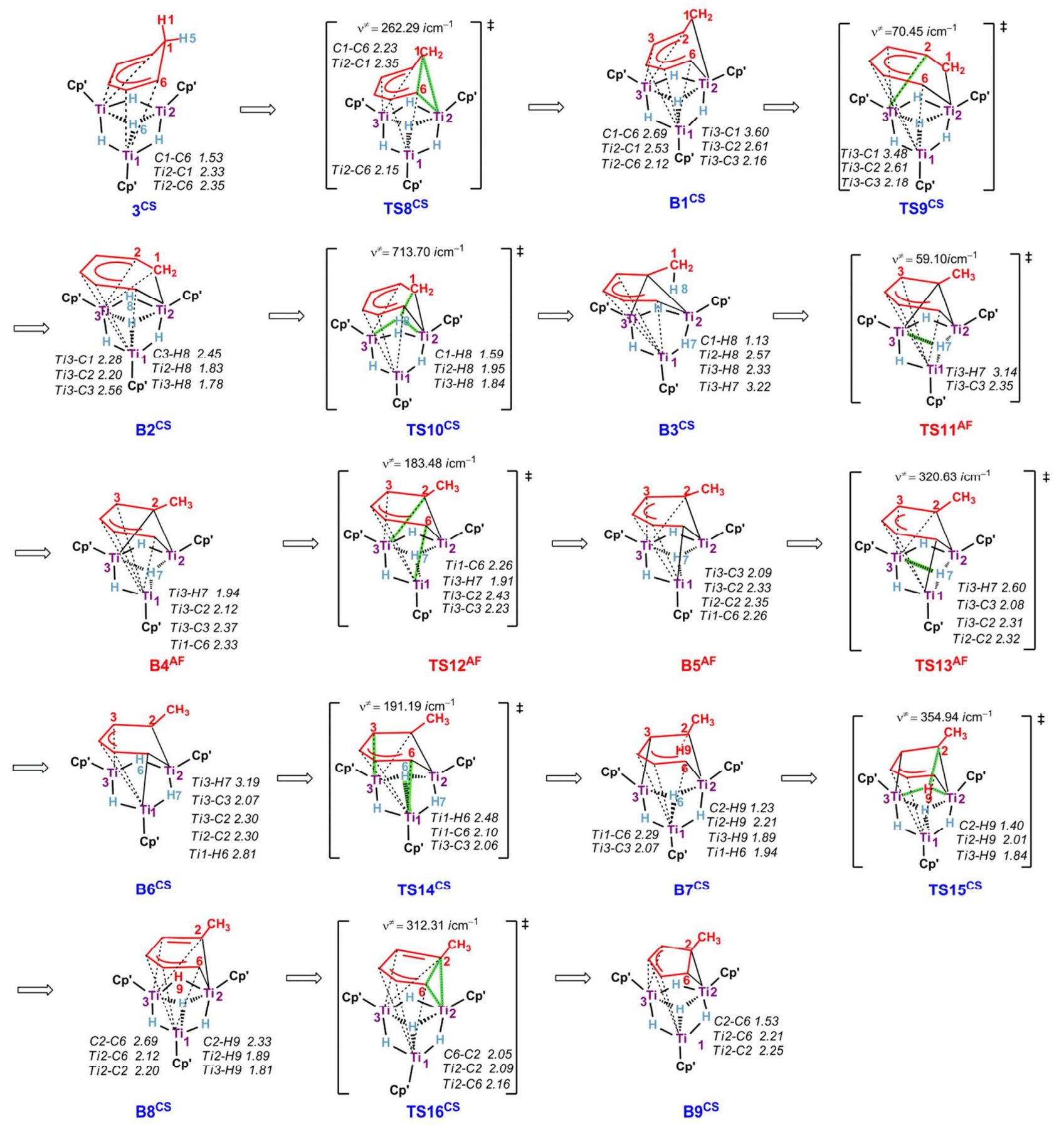

Figure S9. Geometry structures (bond distances in $\AA$ ) of stationary points in Figure 3. 


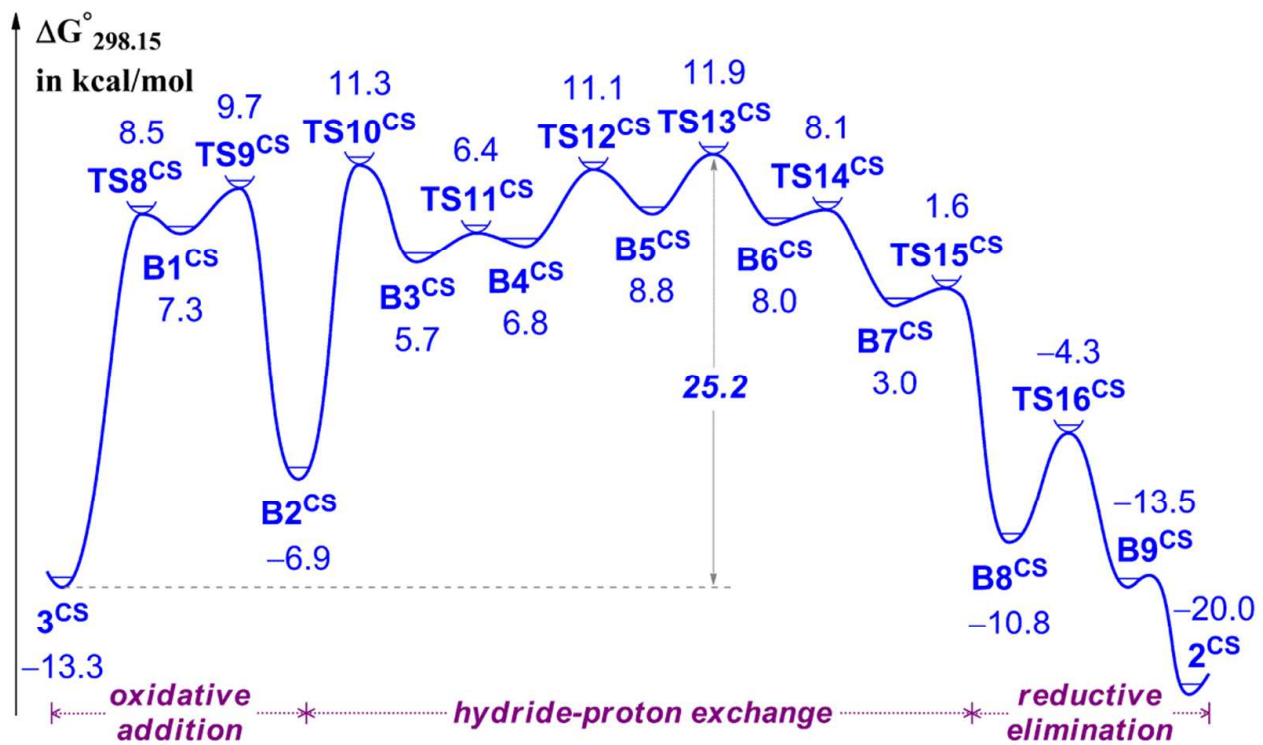

Figure S10. The closed-shell singlet Gibbs energy profile $\left(\Delta \mathrm{G}^{\circ}{ }_{298.15}\right)$ of the reaction $\mathbf{B}$ in Scheme 1 .

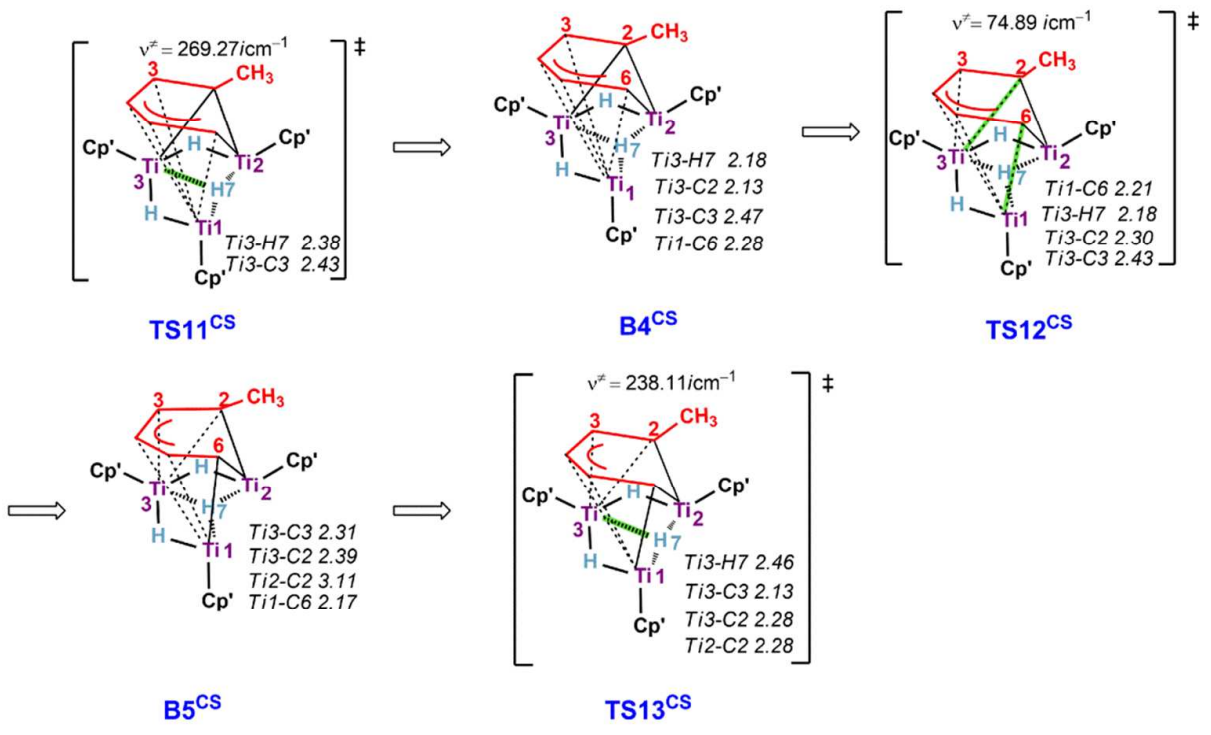

Figure S11. Selected optimized structures for stationary points in Figure S10. 


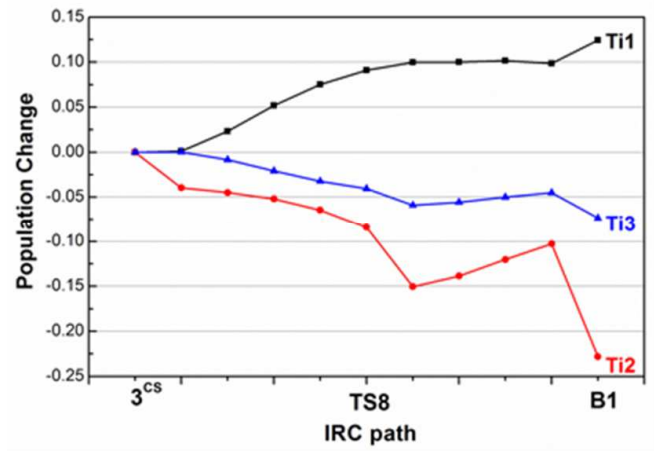

Figure S12. Changes in electron population (in e) of Ti atoms in the C1-C6 bond cleavage.

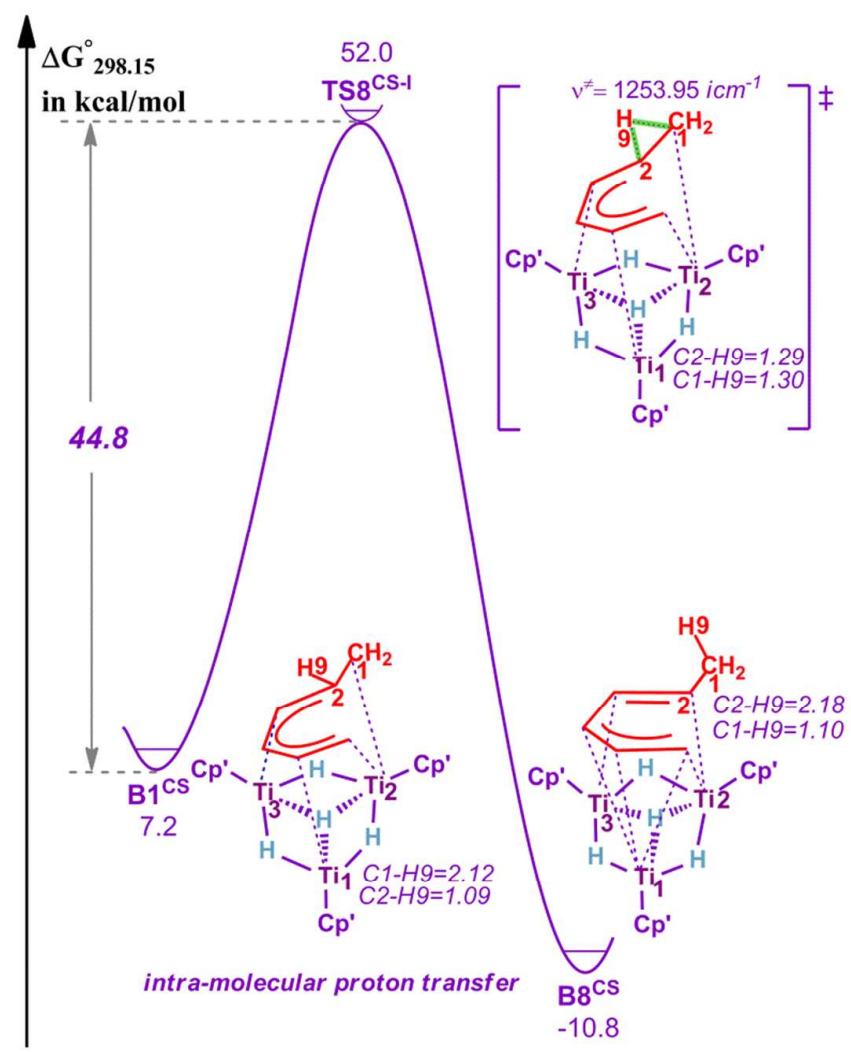

Figure S13. The singlet Gibbs energy profile $\left(\Delta \mathrm{G}_{298.15}^{\circ}\right)$ of intra-molecular proton transfer pathway of $\mathrm{B1}^{\mathrm{CS}} \rightarrow \mathrm{B8}^{\mathrm{CS}}$ transformation. 
Table S9 The Gibbs free energy $(G$, a.u. $)$, Gibbs free energy with the translational entropy correction $\left(G_{S}\right.$, a.u.), electronic energy ( $E_{o p t}$, a.u. $)$, single point energy $\left(E_{s p}\right.$, a.u.), Gibbs free energy with the entropy and single point energy corrections $\left(G_{\text {corr. }}\right.$, a.u. $)$ of all structures except those of Figure S3c.
$G$
$G_{S}$
$E_{\text {opt }}$
$E_{s p}$
$G_{\text {corr: }}$

(U)B3LYP/BSI

(U)B3LYP/BSI

(U)B3LYP/BSI

(U)M06(CPCM)/BSII//

(U)B3LYP/BSI

\begin{tabular}{|c|c|c|c|c|c|}
\hline & & & & & \\
\hline $\mathbf{H}_{2}$ & -1.180336 & -1.17065722 & -1.1789738 & -1.1704715 & -1.16215492 \\
\hline $\mathbf{C}_{6} \mathbf{H}_{6}$ & -232.173014 & -232.1633355 & -232.2486606 & -232.1141045 & -232.0287794 \\
\hline $1^{\mathrm{CS}}$ & -1631.682621 & -1631.67294264 & -1631.9771565 & -1631.5929334 & -1631.28872 \\
\hline $\mathrm{A} 1^{\mathrm{CS}}$ & -1863.844516 & -1863.83483759 & -1864.2275449 & -1863.7187145 & -1863.326007 \\
\hline TS1 $^{\text {CS }}$ & -1863.831593 & -1863.821915 & -1864.212441 & -1863.698311 & -1863.307785 \\
\hline $\mathrm{A} 2^{\mathrm{CS}}$ & -1863.834642 & -1863.824964 & -1864.215361 & -1863.699873 & -1863.309476 \\
\hline $\mathrm{TS2}^{\mathrm{CS}}$ & -1863.817485 & -1863.807807 & -1864.201388 & -1863.688254 & -1863.294672 \\
\hline $\mathrm{A3}^{\mathrm{CS}}$ & -1863.816632 & -1863.806954 & -1864.202864 & -1863.69126 & -1863.295349 \\
\hline TS3 $^{\mathrm{CS}}$ & -1863.798746 & -1863.789068 & -1864.180627 & -1863.673615 & -1863.282056 \\
\hline $\mathrm{A4}^{\mathrm{CS}}$ & -1863.811603 & -1863.801925 & -1864.190167 & -1863.678675 & -1863.290433 \\
\hline TS4 ${ }^{\text {CS }}$ & -1863.809333 & -1863.799655 & -1864.183292 & -1863.665333 & -1863.281696 \\
\hline $\mathrm{A5}^{\mathrm{CS}}$ & -1862.642212 & -1862.632517 & -1863.008871 & -1862.500144 & -1862.12379 \\
\hline TS5 $^{\mathrm{CS}}$ & -1862.624053 & -1862.614358 & -1863.000205 & -1862.503612 & -1862.117765 \\
\hline$A 6^{\mathrm{CS}}$ & -1862.646795 & -1862.637117 & -1863.023505 & -1862.54079 & -1862.154402 \\
\hline TS6 $^{\mathrm{CS}}$ & -1862.645882 & -1862.636204 & -1863.023475 & -1862.540334 & -1862.153063 \\
\hline $\mathbf{A} 7^{\mathrm{CS}}$ & -1862.650004 & -1862.640326 & -1863.030488 & -1862.547246 & -1862.157083 \\
\hline $\mathrm{TS}^{\mathrm{CS}}$ & -1862.643157 & -1862.633479 & -1863.024176 & -1862.542442 & -1862.151745 \\
\hline $\mathrm{As}^{\mathrm{CS}}$ & -1862.665463 & -1862.655785 & -1863.046862 & -1862.555615 & -1862.164538 \\
\hline $3^{\mathrm{CS}}$ & -1862.667742 & -1862.658064 & -1863.049501 & -1862.568051 & -1862.176614 \\
\hline TS8 $^{\mathrm{CS}}$ & -1862.637199 & -1862.627521 & -1863.018909 & -1862.533256 & -1862.141867 \\
\hline $\mathrm{B} 1^{\mathrm{CS}}$ & -1862.642859 & -1862.633181 & -1863.021274 & -1862.531956 & -1862.143862 \\
\hline TS9 ${ }^{C S}$ & -1862.639659 & -1862.629964 & -1863.019494 & -1862.529579 & -1862.140049 \\
\hline $\mathrm{B2}^{\mathrm{CS}}$ & -1862.658553 & -1862.648875 & -1863.040891 & -1862.558374 & -1862.166358 \\
\hline $\operatorname{TS}_{10}{ }^{\mathrm{CS}}$ & -1862.634103 & -1862.624425 & -1863.014318 & -1862.527369 & -1862.137476 \\
\hline $\mathrm{B3}^{\mathrm{CS}}$ & -1862.64556 & -1862.635882 & -1863.026919 & -1862.537358 & -1862.146321 \\
\hline TS11 $^{\mathrm{CS}}$ & -1862.646451 & -1862.636756 & -1863.025774 & -1862.534333 & -1862.145315 \\
\hline $\mathrm{B} 4^{\mathrm{CS}}$ & -1862.645656 & -1862.635961 & -1863.025892 & -1862.534505 & -1862.144575 \\
\hline TS12 $^{\mathrm{CS}}$ & -1862.636445 & -1862.62675 & -1863.019717 & -1862.530774 & -1862.137808 \\
\hline $\mathrm{B5}^{\mathrm{CS}}$ & -1862.638196 & -1862.628501 & -1863.020434 & -1862.533293 & -1862.14136 \\
\hline $\mathrm{TS}_{13}{ }^{\mathrm{CS}}$ & -1862.639714 & -1862.630019 & -1863.019714 & -1862.526106 & -1862.136411 \\
\hline $\mathrm{B6}^{\mathrm{CS}}$ & -1862.644587 & -1862.634892 & -1863.024641 & -1862.532446 & -1862.142697 \\
\hline $\mathrm{TS}_{4}{ }^{\mathrm{CS}}$ & -1862.643807 & -1862.634112 & -1863.024227 & -1862.532616 & -1862.142502 \\
\hline $\mathrm{B} 7^{\mathrm{CS}}$ & -1862.648935 & -1862.639257 & -1863.028881 & -1862.540228 & -1862.150604 \\
\hline TS15 $^{\text {CS }}$ & -1862.648452 & -1862.638774 & -1863.028286 & -1862.542436 & -1862.152924 \\
\hline $\mathrm{B8}^{\mathrm{CS}}$ & -1862.665068 & -1862.65539 & -1863.045773 & -1862.563014 & -1862.17263 \\
\hline TS16 $^{\mathrm{CS}}$ & -1862.65517 & -1862.645492 & -1863.034312 & -1862.55112 & -1862.162301 \\
\hline$B 9^{\mathrm{CS}}$ & -1862.672454 & -1862.662776 & -1863.052994 & -1862.567155 & -1862.176937 \\
\hline $2^{\mathrm{CS}}$ & -1862.683842 & -1862.673979 & -1863.061775 & -1862.575178 & -1862.187381 \\
\hline $\mathbf{1}^{\mathrm{T}}$ & -1631.68964 & -1631.679962 & -1631.981317 & -1631.580337 & -1631.278981 \\
\hline $\mathbf{A} 1^{\mathrm{T}}$ & -1863.852421 & -1863.842743 & -1864.232211 & -1863.706006 & -1863.316538 \\
\hline
\end{tabular}




\begin{tabular}{|c|c|c|c|c|c|}
\hline TS1 $^{\mathrm{T}}$ & -1863.84556 & -1863.835882 & -1864.224497 & -1863.694302 & -1863.305687 \\
\hline $\mathbf{A} 2^{\mathrm{T}}$ & -1863.843438 & -1863.83376 & -1864.225811 & -1863.70129 & -1863.309238 \\
\hline TS2 $^{\mathrm{T}}$ & -1863.842116 & -1863.832438 & -1864.225357 & -1863.701244 & -1863.308325 \\
\hline $\mathbf{A} 3^{T}$ & -1863.844397 & -1863.834719 & -1864.229637 & -1863.707179 & -1863.31226 \\
\hline $\mathbf{T S 3}^{\mathrm{T}}$ & -1863.833531 & -1863.823853 & -1864.214924 & -1863.689284 & -1863.298213 \\
\hline $\mathbf{A} 4^{\mathrm{T}}$ & -1863.834002 & -1863.824307 & -1864.217035 & -1863.700725 & -1863.307997 \\
\hline TS4 $^{\mathrm{T}}$ & -1863.830093 & -1863.820415 & -1864.202878 & -1863.687755 & -1863.305291 \\
\hline $\mathbf{A 5 ^ { T }}$ & -1862.659777 & -1862.650082 & -1863.024227 & -1862.511571 & -1862.137427 \\
\hline TS5 $^{\mathrm{T}}$ & -1862.647503 & -1862.637808 & -1863.019045 & -1862.508918 & -1862.127682 \\
\hline$A 6^{T}$ & -1862.649483 & -1862.639805 & -1863.02307 & -1862.518108 & -1862.134843 \\
\hline TS6 $^{\mathrm{T}}$ & -1862.644625 & -1862.634947 & -1863.018962 & -1862.515881 & -1862.131866 \\
\hline $\mathbf{A} 7^{\mathrm{T}}$ & -1862.647624 & -1862.637946 & -1863.023484 & -1862.521848 & -1862.13631 \\
\hline $\mathbf{T S 7}{ }^{\mathrm{T}}$ & -1862.626543 & -1862.616865 & -1863.00167 & -1862.504015 & -1862.119209 \\
\hline $\mathbf{A} 8^{T}$ & -1862.626174 & -1862.616496 & -1863.002309 & -1862.513095 & -1862.127281 \\
\hline TS8 $^{T}$ & -1862.621272 & -1862.611594 & -1862.996643 & -1862.507187 & -1862.122138 \\
\hline$A 9^{T}$ & -1862.660083 & -1862.650405 & -1863.038218 & -1862.543385 & -1862.155571 \\
\hline $3^{T}$ & -1862.649058 & -1862.63938 & -1863.026728 & -1862.532557 & -1862.145209 \\
\hline TS9 $^{T}$ & -1862.619481 & -1862.609803 & -1862.994896 & -1862.498605 & -1862.113511 \\
\hline $\mathbf{B} 1^{\mathrm{T}}$ & -1862.635373 & -1862.625695 & -1863.011936 & -1862.518556 & -1862.132315 \\
\hline $\mathbf{B} 2^{\mathrm{T}}$ & -1862.634334 & -1862.624656 & -1863.009022 & -1862.514518 & -1862.130152 \\
\hline TS10 $^{T}$ & -1862.626568 & -1862.61689 & -1863.002115 & -1862.509738 & -1862.124513 \\
\hline $\mathbf{B 3}^{\mathrm{T}}$ & -1862.657124 & -1862.647446 & -1863.03506 & -1862.533349 & -1862.145734 \\
\hline $\mathbf{1}^{\mathrm{AF}}$ & -1631.693129 & -1631.683434 & -1631.985479 & -1631.590856 & -1631.288812 \\
\hline $\mathbf{A} 1^{\mathrm{AF}}$ & -1863.855067 & -1863.845372 & -1864.236011 & -1863.715633 & -1863.324995 \\
\hline $\mathrm{TS1}^{\mathrm{AF}}$ & -1863.842977 & -1863.833282 & -1864.225639 & -1863.697124 & -1863.304767 \\
\hline $\mathbf{A} 2^{\mathrm{AF}}$ & -1863.844511 & -1863.834816 & -1864.226104 & -1863.701698 & -1863.31041 \\
\hline $\mathbf{T S 2}^{\mathrm{AF}}$ & -1863.841171 & -1863.831476 & -1864.225489 & -1863.701412 & -1863.307399 \\
\hline $\mathbf{A 3}^{\mathbf{A F}}$ & -1863.843534 & -1863.833839 & -1864.229672 & -1863.706547 & -1863.310714 \\
\hline TS3 $^{\mathrm{AF}}$ & -1863.832166 & -1863.822471 & -1864.214816 & -1863.68846 & -1863.296116 \\
\hline$A 4^{\mathrm{AF}}$ & -1863.840196 & -1863.830501 & -1864.224544 & -1863.70056 & -1863.306517 \\
\hline $\mathrm{TS4}^{\mathrm{AF}}$ & -1863.835078 & -1863.825383 & -1864.211101 & -1863.685671 & -1863.299953 \\
\hline $\mathbf{A 5} \mathbf{5}^{\mathbf{A F}}$ & -1862.667157 & -1862.657462 & -1863.033182 & -1862.508371 & -1862.132651 \\
\hline TS5 $^{\mathrm{AF}}$ & -1862.649885 & -1862.64019 & -1863.022937 & -1862.514788 & -1862.132041 \\
\hline$A 6^{\mathrm{AF}}$ & -1862.652796 & -1862.643101 & -1863.027743 & -1862.527363 & -1862.142722 \\
\hline TS6 $^{\mathrm{AF}}$ & -1862.648934 & -1862.639239 & -1863.026109 & -1862.530915 & -1862.144046 \\
\hline $\mathbf{A} 7^{\mathbf{A F}}$ & -1862.650797 & -1862.641102 & -1863.029011 & -1862.532193 & -1862.144284 \\
\hline $\mathrm{TS}^{\mathrm{AF}}$ & -1862.643239 & -1862.633544 & -1863.018187 & -1862.52128 & -1862.136637 \\
\hline TS11 $^{\mathrm{AF}}$ & -1862.650984 & -1862.64128926 & -1863.028033 & -1862.531651 & -1862.144908 \\
\hline $\mathrm{B4}^{\mathrm{AF}}$ & -1862.658903 & -1862.649208 & -1863.037007 & -1862.5316 & -1862.143802 \\
\hline $\mathrm{TS}_{12}^{\mathrm{AF}}$ & -1862.64207 & -1862.632375 & -1863.023537 & -1862.529849 & -1862.138686 \\
\hline $\mathrm{B5}^{\mathrm{AF}}$ & -1862.65026 & -1862.640565 & -1863.030841 & -1862.537814 & -1862.147538 \\
\hline $\mathrm{TS}_{13}^{\mathrm{AF}}$ & -1862.643215 & -1862.63352 & -1863.021607 & -1862.527038 & -1862.138951 \\
\hline TS8 $^{\text {CS-I }}$ & -1862.559673 & -1862.549995 & -1862.932732 & -1862.455356 & -1862.072619 \\
\hline TS1 $^{\text {CS' }}$ & -1863.807456 & -1863.797761 & -1864.19084 & -1863.686286 & -1863.293207 \\
\hline $\mathrm{A} 2^{\mathrm{CS}}$ & -1863.807355 & -1863.79766 & -1864.190885 & -1863.686721 & -1863.293497 \\
\hline TS1 ${ }^{\text {CS-CH }}$ & -1863.77769 & -1863.768012 & -1864.165772 & -1863.667374 & -1863.269614 \\
\hline
\end{tabular}




\begin{tabular}{|c|c|c|c|c|c|}
\hline $\mathrm{A} 2^{\mathrm{CS}-\mathrm{CH}}$ & -1863.793728 & -1863.78405 & -1864.189008 & -1863.695282 & -1863.290323 \\
\hline$A 1^{\mathrm{CS}-\mathrm{BH}}$ & -1863.826693 & -1863.817015 & -1864.206586 & -1863.692097 & -1863.302526 \\
\hline TS1 $^{\text {BS-CH }}$ & -1863.789569 & -1863.779891 & -1864.17928 & -1863.679683 & -1863.280293 \\
\hline$A 2^{B S-C H}$ & -1863.794174 & -1863.784496 & -1864.18727 & -1863.692278 & -1863.289504 \\
\hline $\mathbf{A} 1_{\mathbf{F}}{ }^{\mathrm{CS}}$ & -1631.668704 & -1631.659009 & -1631.960651 & -1631.570204 & -1631.268562 \\
\hline $\mathrm{TS1}_{\mathrm{F}}{ }^{\mathrm{CS}}$ & -1631.639609 & -1631.629914 & -1631.932978 & -1631.553247 & -1631.250183 \\
\hline$A 2_{F}^{C S}$ & -1631.652448 & -1631.642753 & -1631.948973 & -1631.569455 & -1631.263235 \\
\hline $\mathbf{A 1} 1_{F}^{A F}$ & -1631.677861 & -1631.668166 & -1631.970216 & -1631.568714 & -1631.266664 \\
\hline TS1 $_{F}{ }^{A F}$ & -1631.666473 & -1631.656778 & -1631.955912 & -1631.551276 & -1631.252142 \\
\hline $\mathbf{A} 2_{F}{ }^{A F}$ & -1631.681934 & -1631.672239 & -1631.971481 & -1631.564086 & -1631.264845 \\
\hline
\end{tabular}

Table S10 The Gibbs free energy ( $G$, a.u.), Gibbs free energy with the translational entropy correction $\left(G_{S}\right.$, a.u. $)$, electronic energy $\left(E_{o p t}\right.$, a.u. $)$, single point energy $\left(E_{s p}\right.$, a.u. $)$, Gibbs free energy with the entropy and single point energy corrections $\left(G_{\text {corr: }}\right.$, a.u. $)$ of all structures in Figure S3c.

\begin{tabular}{|c|c|c|c|c|c|}
\hline & $\begin{array}{c}G \\
\text { B3LYP-D3/BSI }\end{array}$ & $\begin{array}{c}G_{S} \\
\text { B3LYP-D3/BSI }\end{array}$ & $\begin{array}{c}E_{o p t} \\
\text { B3LYP-D3/BSI }\end{array}$ & $\begin{array}{c}E_{s p} \\
\text { M06(CPCM)/BSII } \\
\text { // B3LYP-D3/BSI }\end{array}$ & $\begin{array}{c}G_{\text {corr }} \\
\text { M06(CPCM)/BSII// } \\
\text { B3LYP-D3/BSI }\end{array}$ \\
\hline$A 1_{D}^{C S^{\prime}}$ & -1863.931017 & -1863.921322 & -1864.323686 & -1863.723565 & -1863.3212 \\
\hline $\mathrm{TS1}_{\mathrm{D}}{ }^{\mathrm{CS}}$ & -1863.904176 & -1863.894481 & -1864.297104 & -1863.6926 & -1863.289977 \\
\hline$A 2_{D}{ }^{C S^{\prime}}$ & -1863.904256 & -1863.894561 & -1864.298247 & -1863.694013 & -1863.290327 \\
\hline $\mathrm{C}_{6} \mathrm{H}_{6 \mathrm{D}}$ & -232.178099 & -232.1683924 & -232.2537593 & -232.1141045 & -232.0287376 \\
\hline $1_{D}^{C S}$ & -1631.754896 & -1631.74519 & -1632.051309 & -1631.595122 & -1631.289003 \\
\hline
\end{tabular}

Table S11 Translational entropy $\left(S_{t}\right)$, translational entropy correction $\left(S_{t}^{\prime}\right)$, rotational entropy $\left(S_{r}\right)$, vibrational entropy $\left(S_{v}\right)$, enthalpies $(H)$ of all structures.

\begin{tabular}{cccccc}
\hline & $\begin{array}{c}S_{t} \\
(\mathrm{cal} / \mathrm{mol} \cdot \mathrm{K})\end{array}$ & $\begin{array}{c}S_{t}^{\prime} \\
(\mathrm{cal} / \mathrm{mol} \cdot \mathrm{K})\end{array}$ & $\begin{array}{c}S_{r} \\
(\mathrm{cal} / \mathrm{mol} \cdot \mathrm{K})\end{array}$ & $\begin{array}{c}S_{v} \\
(\mathrm{cal} / \mathrm{mol} \cdot \mathrm{K})\end{array}$ & $\begin{array}{c}H \\
(\text { a.u. })\end{array}$ \\
\hline $\mathbf{H}_{\mathbf{2}}$ & 28.08 & 7.70927057 & 3.057 & 0 & -1.165542 \\
$\mathbf{C}_{\mathbf{6}} \mathbf{H}_{\mathbf{6}}$ & 38.979 & 18.6089026 & 20.723 & 4.388 & -232.142563 \\
$\mathbf{1}^{\mathbf{C S}}$ & 44.107 & 23.73716681 & 35.808 & 101.836 & -1631.596265 \\
$\mathbf{A 1}^{\mathbf{C S}}$ & 44.598 & 24.22806146 & 36.942 & 141.382 & -1863.738598 \\
$\mathbf{T S 1}^{\mathbf{C S}}$ & 44.598 & 24.22806146 & 36.903 & 142.03 & -1863.725386 \\
$\mathbf{A 2}^{\mathbf{C S}}$ & 44.598 & 24.22806146 & 36.919 & 145.726 & -1863.726672 \\
$\mathbf{T S 2}^{\mathbf{C S}}$ & 44.598 & 24.22806146 & 36.873 & 137.644 & -1863.713377 \\
$\mathbf{A 3}^{\mathbf{C S}}$ & 44.598 & 24.22806146 & 36.835 & 136.67 & -1863.713005 \\
$\mathbf{T S 3}^{\mathbf{C S}}$ & 44.598 & 24.22806146 & 36.722 & 138.655 & -1863.694228 \\
$\mathbf{A 4}^{\mathbf{C S}}$ & 44.598 & 24.22806146 & 36.844 & 152.758 & -1863.700327 \\
$\mathbf{T S 4}^{\mathbf{C S}}$ & 44.598 & 24.22806146 & 37.038 & 156.133 & -1863.696361 \\
$\mathbf{A 5}^{\mathbf{C S}}$ & 44.586 & 24.21634943 & 36.791 & 141.927 & -1862.536112 \\
$\mathbf{T S 5}^{\mathbf{C S}}$ & 44.586 & 24.21634943 & 36.353 & 122.006 & -1862.527627 \\
$\mathbf{A 6}^{\mathbf{C S}}$ & 44.586 & 24.21634943 & 36.209 & 119.908 & -1862.551434 \\
$\mathbf{T S 6}^{\mathbf{C S}}$ & 44.586 & 24.21634943 & 36.207 & 116.496 & -1862.552143 \\
$\mathbf{A 7}^{\mathbf{C S}}$ & 44.586 & 24.21634943 & 36.171 & 114.483 & -1862.557239 \\
\hline
\end{tabular}




\begin{tabular}{|c|c|c|c|c|c|}
\hline $\mathrm{TS}^{\mathrm{CS}}$ & 44.586 & 24.21634943 & 36.186 & 111.424 & -1862.551838 \\
\hline $\mathrm{As}^{\mathrm{CS}}$ & 44.586 & 24.21634943 & 36.25 & 119.23 & -1862.570405 \\
\hline $3^{\mathrm{CS}}$ & 44.586 & 24.21634943 & 36.192 & 119.292 & -1862.572682 \\
\hline $\mathrm{TS8}^{\mathrm{CS}}$ & 44.586 & 24.21634943 & 36.246 & 115.193 & -1862.544062 \\
\hline $\mathrm{B} 1^{\mathrm{CS}}$ & 44.586 & 24.21634943 & 36.272 & 123.642 & -1862.545694 \\
\hline TS9 $9^{\text {CS }}$ & 44.586 & 24.21634943 & 36.271 & 118.438 & -1862.544967 \\
\hline $\mathrm{B} 2^{\mathrm{CS}}$ & 44.586 & 24.21634943 & 36.272 & 116.123 & -1862.564961 \\
\hline $\mathrm{TS}_{10}{ }^{\mathrm{CS}}$ & 44.586 & 24.21634943 & 36.265 & 118.558 & -1862.539357 \\
\hline $\mathrm{B3}^{\mathrm{CS}}$ & 44.586 & 24.21634943 & 36.267 & 122.913 & -1862.548744 \\
\hline TS11 $^{\text {CS }}$ & 44.586 & 24.21634943 & 36.243 & 124.302 & -1862.548987 \\
\hline $\mathrm{B} 4^{\mathrm{CS}}$ & 44.586 & 24.21634943 & 36.232 & 125.089 & -1862.547822 \\
\hline $\mathrm{TS}_{12}{ }^{\mathrm{CS}}$ & 44.586 & 24.21634943 & 36.229 & 117.447 & -1862.542243 \\
\hline $\mathrm{B5}^{\mathrm{CS}}$ & 44.586 & 24.21634943 & 36.229 & 121.129 & -1862.542246 \\
\hline $\mathrm{TS}_{13}{ }^{\mathrm{CS}}$ & 44.586 & 24.21634943 & 36.25 & 121.139 & -1862.543749 \\
\hline $\mathrm{B6}^{\mathrm{CS}}$ & 44.586 & 24.21634943 & 36.256 & 125.092 & -1862.54674 \\
\hline TS14 ${ }^{\text {CS }}$ & 44.586 & 24.21634943 & 36.253 & 122.332 & -1862.547274 \\
\hline $\mathrm{B} 7^{\mathrm{CS}}$ & 44.586 & 24.21634943 & 36.314 & 120.116 & -1862.553426 \\
\hline $\mathrm{TS}_{15}^{\mathrm{CS}}$ & 44.586 & 24.21634943 & 36.299 & 116.314 & -1862.554756 \\
\hline $\mathrm{B8}^{\mathrm{CS}}$ & 44.586 & 24.21634943 & 36.259 & 118.005 & -1862.570587 \\
\hline TS16 $^{\text {CS }}$ & 44.586 & 24.21634943 & 36.228 & 118.281 & -1862.560574 \\
\hline B9 $9^{\mathrm{CS}}$ & 44.586 & 24.21634943 & 36.219 & 120.114 & 1862.576991 \\
\hline $2^{\mathrm{CS}}$ & 44.586 & 24.21634943 & 36.296 & 124.371 & -1862.586319 \\
\hline $\mathbf{1}^{\mathrm{T}}$ & 44.107 & 23.73716681 & 35.904 & 104.352 & -1631.601005 \\
\hline $\mathbf{A 1}{ }^{\mathrm{T}}$ & 44.598 & 24.22806146 & 36.987 & 144.983 & -1863.743735 \\
\hline TS1 $^{T}$ & 44.598 & 24.22806146 & 36.982 & 143.383 & -1863.737635 \\
\hline$A 2^{T}$ & 44.598 & 24.22806146 & 36.815 & 140.172 & -1863.737119 \\
\hline $\mathbf{T S 2}^{\mathrm{T}}$ & 44.598 & 24.22806146 & 36.767 & 136.367 & -1863.737627 \\
\hline $\mathbf{A} 3^{T}$ & 44.598 & 24.22806146 & 36.798 & 134.818 & -1863.740629 \\
\hline $\mathbf{T S 3}^{\mathrm{T}}$ & 44.598 & 24.22806146 & 36.822 & 138.613 & -1863.727949 \\
\hline$A 4^{T}$ & 44.598 & 24.22806146 & 36.859 & 144.659 & -1863.740774 \\
\hline TS4 ${ }^{T}$ & 44.598 & 24.22806146 & 36.681 & 155.272 & -1863.716663 \\
\hline $\mathbf{A 5 ^ { T }}$ & 44.586 & 24.21634943 & 36.69 & 144.012 & -1862.568598 \\
\hline TS5 $^{\mathrm{T}}$ & 44.586 & 24.21634943 & 36.392 & 126.087 & -1862.548083 \\
\hline$A 6^{T}$ & 44.586 & 24.21634943 & 36.351 & 125.019 & -1862.55059 \\
\hline TS6 $^{\text {T }}$ & 44.586 & 24.21634943 & 36.335 & 122.456 & -1862.546957 \\
\hline $\mathbf{A} 7^{\mathrm{T}}$ & 44.586 & 24.21634943 & 36.359 & 121.614 & -1862.550344 \\
\hline $\mathrm{TS7}^{\mathrm{T}}$ & 44.586 & 24.21634943 & 36.269 & 115.915 & -1862.532014 \\
\hline$A 8^{T}$ & 44.586 & 24.21634943 & 36.235 & 116.934 & -1862.531177 \\
\hline TS8 $^{T}$ & 44.586 & 24.21634943 & 36.256 & 115.17 & -1862.527102 \\
\hline$A 9^{T}$ & 44.586 & 24.21634943 & 36.282 & 122.299 & -1862.562515 \\
\hline $3^{T}$ & 44.586 & 24.21634943 & 36.261 & 122.611 & -1862.551351 \\
\hline TS9 $^{\mathrm{T}}$ & 44.586 & 24.21634943 & 36.269 & 122.054 & -1862.522035 \\
\hline$B 1^{T}$ & 44.586 & 24.21634943 & 36.282 & 121.742 & -1862.538069 \\
\hline $\mathbf{B} 2^{\mathrm{T}}$ & 44.586 & 24.21634943 & 36.317 & 125.259 & -1862.535342 \\
\hline TS10 $^{T}$ & 44.586 & 24.21634943 & 36.305 & 120.818 & -1862.529692 \\
\hline $\mathbf{B 3}^{\mathrm{T}}$ & 44.586 & 24.21634943 & 36.289 & 124.744 & -1862.55839 \\
\hline
\end{tabular}




\begin{tabular}{|c|c|c|c|c|c|}
\hline $\mathbf{1}^{\mathrm{AF}}$ & 44.107 & 23.73716681 & 35.865 & 105.724 & -1631.604898 \\
\hline $\mathbf{A} 1^{\mathbf{A F}}$ & 44.598 & 24.22806146 & 36.983 & 145.097 & -1863.747365 \\
\hline TS1 $^{\mathrm{AF}}$ & 44.598 & 24.22806146 & 36.969 & 138.336 & -1863.738494 \\
\hline $\mathbf{A} 2^{\mathbf{A F}}$ & 44.598 & 24.22806146 & 36.828 & 143.887 & -1863.737458 \\
\hline TS2 $^{\mathrm{AF}}$ & 44.598 & 24.22806146 & 36.769 & 136.337 & -1863.737733 \\
\hline $\mathbf{A 3}^{\mathbf{A F}}$ & 44.598 & 24.22806146 & 36.192 & 119.291 & -1862.572682 \\
\hline $\mathrm{TS3}^{\mathrm{AF}}$ & 44.598 & 24.22806146 & 36.821 & 138.166 & -1863.727835 \\
\hline $\mathrm{A4}^{\mathrm{AF}}$ & 44.598 & 24.22806146 & 36.807 & 141.059 & -1863.734496 \\
\hline $\mathrm{TS}^{\mathrm{AF}}$ & 44.598 & 24.22806146 & 36.675 & 151.025 & -1863.724706 \\
\hline $\mathbf{A} 5^{\mathrm{AF}}$ & 44.586 & 24.21634943 & 36.668 & 139.986 & -1862.562039 \\
\hline TS5 $^{\mathrm{AF}}$ & 44.586 & 24.21634943 & 36.297 & 119.511 & -1862.55372 \\
\hline$A 6^{\mathrm{AF}}$ & 44.586 & 24.21634943 & 36.326 & 125.533 & -1862.554707 \\
\hline TS6 $^{\mathbf{A F}}$ & 44.586 & 24.21634943 & 36.207 & 116.482 & -1862.552142 \\
\hline $\mathbf{A} 7^{\mathbf{A F}}$ & 44.586 & 24.21634943 & 36.333 & 120.31 & -1862.555186 \\
\hline $\mathrm{TS}^{\mathrm{AF}}$ & 44.586 & 24.21634943 & 36.355 & 121.355 & -1862.547121 \\
\hline TS11 $^{\mathrm{AF}}$ & 44.586 & 24.21634943 & 36.33 & 126.65 & -1862.552362 \\
\hline $\mathrm{B} 4^{\mathrm{AF}}$ & 44.586 & 24.21634943 & 36.344 & 128.473 & -1862.559408 \\
\hline $\mathrm{TS}_{12}^{\mathrm{AF}}$ & 44.586 & 24.21634943 & 36.262 & 118.863 & -1862.54718 \\
\hline $\mathbf{B 5}^{\mathrm{AF}}$ & 44.586 & 24.21634943 & 36.245 & 122.675 & -1862.553568 \\
\hline TS13 $^{\mathrm{AF}}$ & 44.586 & 24.21634943 & 36.236 & 124.994 & -1862.545425 \\
\hline TS8 $^{\text {CS-I }}$ & 44.586 & 24.21634943 & 36.293 & 120.259 & -1862.464106 \\
\hline TS1 $^{\text {CS-CH }}$ & 44.598 & 24.22806146 & 36.456 & 126.084 & -1863.679272 \\
\hline $\mathrm{A} 2^{\mathrm{CS}-\mathrm{CH}}$ & 44.598 & 24.22806146 & 36.37 & 124.188 & -1863.696252 \\
\hline$A 1^{\text {CS-BH }}$ & 44.598 & 24.22806146 & 36.983 & 145.154 & -1863.718964 \\
\hline TS1 $^{\text {BS-CH }}$ & 44.598 & 24.22806146 & 36.498 & 125.424 & -1863.691444 \\
\hline $\mathrm{A} 2^{\mathrm{BS}-\mathrm{CH}}$ & 44.598 & 24.22806146 & 36.479 & 126.018 & -1863.695776 \\
\hline TS1 $^{\text {CS' }}$ & 44.598 & 24.19338785 & 36.626 & 137.576 & -1863.703497 \\
\hline $\mathbf{A} 2^{\mathrm{CS}^{\prime}}$ & 44.598 & 24.19338785 & 36.611 & 139.369 & -1863.702551 \\
\hline$A 1_{D}{ }^{C S^{\prime}}$ & 44.598 & 24.19338785 & 36.437 & 125.694 & -1863.832794 \\
\hline $\mathrm{TS1}_{\mathrm{D}}{ }^{\mathrm{CS}}$ & 44.598 & 24.19338785 & 36.382 & 123.569 & -1863.806988 \\
\hline $\mathbf{A} 2_{\mathrm{D}} \mathrm{CS}^{\prime}$ & 44.598 & 24.19338785 & 36.319 & 124.354 & -1863.806725 \\
\hline $\mathrm{C}_{6} \mathrm{H}_{6 \mathrm{D}}$ & 38.979 & 18.54980599 & 20.723 & 4.393 & -232.147645 \\
\hline $1_{D}^{C S}$ & 44.107 & 23.6780702 & 35.711 & 101.498 & -1631.668746 \\
\hline$A 1_{F}^{C S}$ & 44.107 & 23.6780702 & 35.806 & 106.649 & -1631.580063 \\
\hline $\mathrm{TS1}_{\mathrm{F}}{ }^{\mathrm{CS}}$ & 44.107 & 23.6780702 & 35.66 & 100.691 & -1631.553867 \\
\hline $\mathrm{A} 2_{\mathrm{F}}{ }^{\mathrm{CS}}$ & 44.107 & 23.6780702 & 35.605 & 102.044 & -1631.56609 \\
\hline$A 1_{F}^{A F}$ & 44.107 & 23.6780702 & 35.882 & 105.398 & -1631.589777 \\
\hline $\mathbf{T S 1}_{\mathbf{F}}{ }^{\mathrm{AF}}$ & 44.107 & 23.6780702 & 35.897 & 107.43 & -1631.577417 \\
\hline $\mathbf{A} 2_{\mathrm{F}}{ }^{\mathbf{A F}}$ & 44.107 & 23.6780702 & 35.891 & 112.619 & -1631.590415 \\
\hline
\end{tabular}


Table S12 The spin density of titanium atoms in the triplet and antiferromagnetic singlet structures optimized at the UB3LYP/BSI level.

\begin{tabular}{|c|c|c|c|}
\hline & Ti1 & Ti2 & Ti3 \\
\hline $1^{T}$ & 1.024698 & 1.023906 & 0.010073 \\
\hline $\mathbf{A} 1^{\mathrm{T}}$ & 1.063744 & 1.037275 & -0.004584 \\
\hline $\mathbf{T S 1}^{\mathrm{T}}$ & 1.075082 & -0.028767 & 1.09763 \\
\hline $\mathbf{A} 2^{\mathrm{T}}$ & 1.071463 & -0.029254 & 1.104704 \\
\hline $\mathbf{T S 2}^{\mathrm{T}}$ & 1.085306 & 1.09524 & -0.047868 \\
\hline $\mathbf{A} 3^{T}$ & 1.08433 & -0.058145 & 1.083159 \\
\hline $\mathbf{T S 3}^{\mathrm{T}}$ & 1.119284 & -0.070779 & 1.068729 \\
\hline$A 4^{T}$ & 1.236498 & 0.665754 & 0.252414 \\
\hline $\mathrm{TS4}^{\mathrm{T}}$ & 1.24096 & 0.530302 & 0.399122 \\
\hline$A 5^{T}$ & 1.210983 & 0.594764 & 0.367632 \\
\hline $\mathbf{T S 5}^{\mathrm{T}}$ & 1.024541 & 1.036396 & -0.117798 \\
\hline$A 6^{T}$ & 1.051667 & 1.043966 & 0.057077 \\
\hline TS6 $^{\mathrm{T}}$ & 0.96211 & 1.063028 & 0.058639 \\
\hline $\mathbf{A} 7^{\mathrm{T}}$ & 1.011853 & 1.097459 & -0.059498 \\
\hline $\mathrm{TS}^{\mathrm{T}}$ & 1.019494 & 0.992899 & 0.192456 \\
\hline$A 8^{T}$ & 0.958769 & 0.686086 & 0.697356 \\
\hline $\mathbf{T S 8}^{\mathrm{T}}$ & 1.054386 & 0.918579 & 0.318287 \\
\hline$A 9^{T}$ & 0.060108 & 1.603207 & 0.368492 \\
\hline $3^{T}$ & 0.526557 & 1.079941 & 0.5071 \\
\hline TS9 $^{T}$ & 0.195381 & 0.914522 & 1.039274 \\
\hline $\mathbf{B} 1^{\mathrm{T}}$ & 0.394919 & 0.206592 & 1.501366 \\
\hline $\mathbf{B} 2^{\mathrm{T}}$ & 0.257785 & 1.036821 & 0.910619 \\
\hline $\operatorname{TS}_{10}{ }^{T}$ & 0.298535 & 1.095743 & 0.818898 \\
\hline $\mathbf{B 3}^{\mathrm{T}}$ & 0.213676 & 0.990689 & 1.08158 \\
\hline $\mathbf{1}^{\mathrm{AF}}$ & 0.981328 & -0.988011 & 0.000608 \\
\hline $\mathbf{A} 1^{\mathbf{A F}}$ & 1.031911 & -0.01542 & -0.98406 \\
\hline TS1 $^{\mathrm{AF}}$ & 1.118253 & 0.002875 & -1.130367 \\
\hline $\mathbf{A} 2^{\mathbf{A F}}$ & 1.132774 & -0.033876 & -1.125046 \\
\hline $\mathrm{TS2}^{\mathrm{AF}}$ & 1.154236 & -0.058159 & -1.108926 \\
\hline $\mathbf{A 3}^{\mathrm{AF}}$ & 1.091573 & -0.037672 & -1.102912 \\
\hline $\mathbf{T S 3}^{\mathrm{AF}}$ & 1.116147 & -0.046932 & -1.112398 \\
\hline$A 4^{\mathrm{AF}}$ & 0.882058 & 0.230421 & -1.19646 \\
\hline $\mathrm{TS}^{\mathrm{AF}}$ & 0.031873 & -1.143232 & 1.086461 \\
\hline $\mathbf{A 5} \mathbf{5}^{\mathbf{A F}}$ & 1.14111 & -1.178553 & 0.041256 \\
\hline TS5 $^{\mathrm{AF}}$ & -0.340637 & 1.301191 & -1.043787 \\
\hline$A 6^{\mathrm{AF}}$ & 0.020524 & -1.05174 & 1.008947 \\
\hline TS6 $^{\mathrm{AF}}$ & 0.640242 & -1.064856 & 0.423218 \\
\hline $\mathbf{A} 7^{\mathbf{A F}}$ & 0.951848 & -1.050084 & 0.014906 \\
\hline $\mathrm{TS} 7^{\mathrm{AF}}$ & 0.94332 & -1.039626 & -0.016762 \\
\hline TS11 $^{\mathrm{AF}}$ & -0.093506 & 0.80896 & -0.702405 \\
\hline $\mathrm{B} 4^{\mathrm{AF}}$ & -1.036728 & 1.127106 & -0.047565 \\
\hline $\mathrm{TS}_{12}{ }^{\mathrm{AF}}$ & 0.672756 & -1.165195 & 0.329396 \\
\hline
\end{tabular}




\begin{tabular}{cccc}
\hline $\mathbf{B 5}^{\mathbf{A F}}$ & 0.845458 & -0.86637 & -0.116433 \\
$\mathbf{T S 1 3}^{\mathbf{A F}}$ & 0.812258 & -0.820851 & 0.038532 \\
\hline
\end{tabular}

Table $\mathbf{S 1 3}$ The relative Gibbs free energy before $(\Delta G, \mathrm{kcal} / \mathrm{mol})$ and after entropy correction $\left(\Delta G_{\text {corr. }}\right.$ $\mathrm{kcal} / \mathrm{mol})$ at the (U)M06(CPCM)/BSII//(U)B3LYP/BSI level.

\begin{tabular}{|c|c|c|c|c|c|c|c|c|c|}
\hline & $\mathbf{1}^{\mathrm{AF}}$ & $1^{\mathrm{CS}}$ & $1^{T}$ & $\mathbf{A} 1^{\mathrm{AF}}$ & $\mathrm{A} 1^{\mathrm{CS}}$ & $\mathbf{A} 1^{T}$ & TS1 $^{\mathrm{AF}}$ & TS1 $^{\text {CS }}$ & $\operatorname{TS1}^{\mathrm{T}}$ \\
\hline$\Delta G$ & 0.0 & 0.1 & 6.2 & 1.4 & 0.8 & 6.7 & 14.1 & 12.2 & 13.6 \\
\hline \multirow[t]{2}{*}{$\Delta G_{\text {corr }}$} & 0.0 & 0.1 & 6.2 & -4.6 & -5.3 & 0.7 & 8.0 & 6.2 & 7.5 \\
\hline & $A 2^{\mathrm{AF}}$ & $\mathrm{A} 2^{\mathrm{CS}}$ & $\mathbf{A} 2^{T}$ & TS2 $^{\mathrm{AF}}$ & TS2 $^{\mathrm{CS}}$ & $\mathbf{T S 2}^{\mathrm{T}}$ & $\mathbf{A} 3^{\mathrm{AF}}$ & $A 3^{\mathrm{CS}}$ & $\mathbf{A 3}^{\mathrm{T}}$ \\
\hline$\Delta G$ & 10.6 & 11.2 & 11.3 & 12.5 & 20.5 & 11.9 & 10.4 & 20.0 & 9.4 \\
\hline \multirow[t]{2}{*}{$\Delta G_{\text {corr }}$} & 4.5 & 5.1 & 5.2 & 6.4 & 14.4 & 5.8 & 4.3 & 14.0 & 3.3 \\
\hline & TS3 $^{\mathrm{AF}}$ & $\mathrm{TS3}^{\mathrm{CS}}$ & $\mathbf{T S 3}^{\mathrm{T}}$ & $\mathrm{A} 4^{\mathrm{AF}}$ & $A 4^{C S}$ & $A 4^{T}$ & TS4 $^{\mathrm{AF}}$ & $\mathrm{TS}^{\mathrm{CS}}$ & $\mathbf{T S 4}^{\mathrm{T}}$ \\
\hline$\Delta G$ & 19.5 & 28.4 & 18.2 & 13.0 & 23.1 & 12.1 & 17.1 & 28.6 & 13.8 \\
\hline \multirow[t]{2}{*}{$\Delta G_{\text {corr }}$} & 13.5 & 22.3 & 12.2 & 6.9 & 17.0 & 6.0 & 11.1 & 22.5 & 7.7 \\
\hline & $A 5^{A F}$ & $A 5^{\mathrm{CS}}$ & $A 5^{T}$ & TS5 $^{\mathrm{AF}}$ & TS5 $^{\mathrm{CS}}$ & $\mathbf{T S 5}^{\mathrm{T}}$ & $A 6^{\mathrm{AF}}$ & $A 6^{\mathrm{CS}}$ & $A 6^{T}$ \\
\hline$\Delta G$ & 14.3 & 19.9 & 11.3 & 14.7 & 23.6 & 17.4 & 8.0 & 0.6 & 12.9 \\
\hline \multirow[t]{2}{*}{$\Delta G_{\text {corr: }}$} & 14.3 & 19.9 & 11.3 & 14.7 & 23.6 & 17.4 & 8.0 & 0.6 & 12.9 \\
\hline & TS6 $^{\mathbf{A F}}$ & TS6 $^{\mathrm{CS}}$ & TS6 $^{\mathrm{T}}$ & $\mathbf{A} 7^{\mathrm{AF}}$ & $\mathrm{A} 7^{\mathrm{CS}}$ & $A 7^{T}$ & $\mathrm{TS}^{\mathrm{AF}}$ & $\mathrm{TS}^{\mathrm{CS}}$ & $\mathbf{T S} 7^{\mathrm{T}}$ \\
\hline$\Delta G$ & 7.1 & 1.5 & 14.8 & 7.0 & -1.0 & 12.0 & 11.8 & 2.3 & 22.7 \\
\hline \multirow[t]{2}{*}{$\Delta G_{\text {corr }}$} & 7.1 & 1.5 & 14.8 & 7.0 & -1.0 & 12.0 & 11.8 & 2.3 & 22.7 \\
\hline & $\mathrm{As}^{\mathrm{CS}}$ & $\mathbf{A 8}^{\mathrm{T}}$ & TS8 $^{T}$ & $A 9^{T}$ & $3^{\mathrm{CS}}$ & $3^{T}$ & $\mathrm{TS8}^{\mathrm{CS}}$ & TS9 $^{T}$ & $\mathrm{~B} 1^{\mathrm{CS}}$ \\
\hline$\Delta G$ & -5.7 & 17.7 & 20.9 & -0.1 & -13.3 & 6.4 & 8.5 & 26.3 & 7.3 \\
\hline \multirow[t]{2}{*}{$\Delta G_{\text {corr }}$} & -5.7 & 17.7 & 20.9 & -0.1 & -13.3 & 6.4 & 8.5 & 26.3 & 7.3 \\
\hline & $B 1^{T}$ & TS9 $^{\text {CS }}$ & $\mathrm{B} 2^{\mathrm{CS}}$ & $B 2^{T}$ & TS10 $^{\mathrm{CS}}$ & TS10 $^{T}$ & $\mathrm{B3}^{\mathrm{CS}}$ & $\mathrm{B3}^{\mathrm{T}}$ & TS11 $^{\text {AF }}$ \\
\hline$\Delta G$ & 14.5 & 9.7 & -6.9 & 15.9 & 11.3 & 19.4 & 5.7 & 6.1 & 6.6 \\
\hline \multirow[t]{2}{*}{$\Delta G_{\text {corr }}$} & 14.5 & 9.7 & -6.9 & 15.9 & 11.3 & 19.4 & 5.7 & 6.1 & 6.6 \\
\hline & TS11 $^{\mathrm{CS}}$ & $B 4^{\mathrm{AF}}$ & $\mathrm{B} 4^{\mathrm{CS}}$ & $\mathrm{TS12}^{\mathrm{AF}}$ & $\mathrm{TS}_{12}{ }^{\mathrm{CS}}$ & $\mathrm{B5}^{\mathrm{AF}}$ & $\mathrm{B5}^{\mathrm{CS}}$ & $\mathrm{TS}_{13}^{\mathrm{AF}}$ & $\mathrm{TS}{ }^{\mathrm{CS}}$ \\
\hline$\Delta G$ & 6.4 & 7.3 & 6.8 & 10.5 & 11.1 & 5.0 & 8.8 & 10.3 & 11.9 \\
\hline \multirow[t]{2}{*}{$\Delta G_{\text {corr }}$} & 6.4 & 7.3 & 6.8 & 10.5 & 11.1 & 5.0 & 8.8 & 10.3 & 11.9 \\
\hline & $\mathrm{B6}^{\mathrm{CS}}$ & $\mathrm{TS}_{14}{ }^{\mathrm{CS}}$ & $\mathrm{B}^{\mathrm{CS}}$ & $\mathrm{TS}^{\mathrm{CS}}$ & $\mathrm{B8}^{\mathrm{CS}}$ & TS16 $^{\mathrm{CS}}$ & B9 ${ }^{\mathrm{CS}}$ & $2^{\mathrm{CS}}$ & $A 1_{F}{ }^{C S}$ \\
\hline
\end{tabular}




\begin{tabular}{|c|c|c|c|c|c|c|c|c|c|}
\hline$\Delta G$ & 8.0 & 8.1 & 3.0 & 1.6 & -10.8 & -4.3 & -13.5 & -20.0 & 12.7 \\
\hline \multirow[t]{2}{*}{$\Delta G_{\text {corr }}$} & 8.0 & 8.1 & 3.0 & 1.6 & -10.8 & -4.3 & -13.5 & -20.0 & 12.7 \\
\hline & $\mathrm{TS1}_{\mathrm{F}}{ }^{\mathrm{CS}}$ & $\mathrm{A} 2_{\mathrm{F}}{ }^{\mathrm{CS}}$ & $\mathrm{TS1}^{\mathrm{CS}}$ & $A 2^{\mathrm{CS}^{\prime}}$ & $\mathbf{A} 1_{F}^{A F}$ & $\mathrm{TS1}_{\mathrm{F}}^{\mathrm{AF}}$ & $A 2_{F}{ }^{A F}$ & $\mathrm{TS1}^{\mathrm{CS}-\mathrm{CH}}$ & $\mathrm{A} 2^{\mathrm{CS}-\mathrm{CH}}$ \\
\hline$\Delta G$ & 24.2 & 16.0 & 21.4 & 21.2 & 13.9 & 23.0 & 15.0 & 42.3 & 29.3 \\
\hline \multirow[t]{2}{*}{$\Delta G_{\text {corr }}$} & 24.2 & 16.0 & 15.3 & 15.1 & 13.9 & 23.0 & 15.0 & 30.1 & 17.1 \\
\hline & $A 1^{C S-B H}$ & TS1 ${ }^{\text {CS-BH }}$ & $A 2^{\mathrm{CS}-\mathrm{BH}}$ & $A 1_{D}{ }^{C S^{\prime}}$ & $\mathbf{T S 1} 1_{D}{ }^{C S^{\prime}}$ & $A 2_{D}{ }^{C S^{\prime}}$ & TS8 $^{\text {CS-I }}$ & & \\
\hline$\Delta G$ & 21.6 & 35.6 & 29.8 & 3.9 & 23.5 & 23.3 & 52.0 & & \\
\hline$\Delta G_{\text {corr }}$ & 9.5 & 23.4 & 17.6 & -2.2 & 17.4 & 17.2 & 52.0 & & \\
\hline
\end{tabular}




\section{Coordinates of All Geometry Optimized Structures}

$\mathrm{H}_{2}$

$\mathrm{H}$

$\mathrm{H}$

$\mathrm{C}_{6} \mathrm{H}_{6}$

$\mathrm{C}$

C

C

$\mathrm{C}$

C

C

$\mathrm{H}$

$\mathrm{H}$

$\mathrm{H}$

$\mathrm{H}$

$\mathrm{H}$

$\mathrm{H}$

$1^{\mathrm{CS}}$

Ti

$\mathrm{Ti}$

$\mathrm{Ti}$

$\mathrm{Si}$

$\mathrm{Si}$

$\mathrm{Si}$

C

C

C

C

C

C

C

C

C

C

C

C

C

C

C

$\mathrm{H}$

$\mathrm{H}$

$\mathrm{H}$

$\mathrm{H}$ $\begin{array}{rrr}0.00000000 & 0.00000000 & 0.37184200 \\ 0.00000000 & 0.00000000 & -0.37184200\end{array}$

$\begin{array}{rrc}0.00000000 & 1.39649000 & 0.00000000 \\ 1.20939600 & 0.69824500 & 0.00000000 \\ 1.20939600 & -0.69824500 & 0.00000000 \\ 0.00000000 & -1.39649000 & 0.00000000 \\ -1.20939600 & -0.69824500 & 0.00000000 \\ -1.20939600 & 0.69824500 & 0.00000000 \\ 0.00000000 & 2.48319100 & 0.00000000 \\ 2.15050600 & 1.24159500 & 0.00000000 \\ 2.15050600 & -1.24159500 & 0.00000000 \\ 0.00000000 & -2.48319100 & 0.00000000 \\ -2.15050600 & -1.24159500 & 0.00000000 \\ -2.15050600 & 1.24159500 & 0.00000000\end{array}$

$\begin{array}{lll}0.22464100 & 1.29479900 & -0.47816700\end{array}$

$\begin{array}{lll}-1.27386200 & -0.79426800 & -0.59605700\end{array}$

$\begin{array}{lll}1.09754200 & -0.96914900 & 0.39015000\end{array}$

$\begin{array}{lll}-4.23756100 & -1.11912800 & 1.66490300\end{array}$

$\begin{array}{lll}-0.53782000 & 4.05744600 & 1.93153800\end{array}$

$\begin{array}{lll}4.53252500 & -1.22758200 & -1.00949500\end{array}$

$-3.48681300 \quad-1.44551300 \quad-0.01462100$

$-2.77434100 \quad-2.61724500 \quad-0.42683400$

$\begin{array}{lll}-2.42861600 & -2.49677100 & -1.79771000\end{array}$

$\begin{array}{lll}-2.91793500 & -1.24774300 & -2.25971300\end{array}$

$\begin{array}{lll}-3.56383000 & -0.60560000 & -1.17127500\end{array}$

$\begin{array}{lll}0.10262400 & 3.55747400 & 0.24887500\end{array}$

$\begin{array}{lll}1.46072700 & 3.27959500 & -0.10932400\end{array}$

$\begin{array}{lll}1.53094300 & 3.00324100 & -1.49934700\end{array}$

$\begin{array}{lll}0.21793800 & 3.10293400 & -2.02776700\end{array}$

$\begin{array}{lll}-0.65463100 & 3.44030400 & -0.96050200\end{array}$

$\begin{array}{lll}3.33604800 & -1.73787100 & 0.33371600\end{array}$

$\begin{array}{lll}2.48310400 & -2.88498400 & 0.33284100\end{array}$

$\begin{array}{lll}1.77811300 & -2.93640100 & 1.56362200\end{array}$

$2.18164800 \quad-1.82420200 \quad 2.34880100$

$\begin{array}{lll}3.13325600 & -1.09090900 & 1.59649800\end{array}$

$\begin{array}{lll}1.39920200 & 0.77791100 & 0.82375000\end{array}$

$\begin{array}{lll}1.56131600 & 0.13691600 & -0.99571600\end{array}$

$\begin{array}{lll}-1.57418500 & 0.99874900 & -0.41291000\end{array}$

$\begin{array}{lll}-0.48021500 & 0.29827600 & -1.85074500\end{array}$ 


$\begin{array}{lrrr}\mathrm{H} & -0.48101200 & -1.83609800 & 0.67813700 \\ \mathrm{H} & 0.26995000 & -1.66656500 & -1.09772200 \\ \mathrm{H} & -0.43021200 & 0.10210000 & 0.89168800 \\ \mathrm{H} & -5.62361000 & -1.66237700 & 1.74372700 \\ \mathrm{H} & -3.42901300 & -1.78047300 & 2.72232300 \\ \mathrm{H} & -4.30421500 & 0.34361800 & 1.91723000 \\ \mathrm{H} & -2.52213300 & -3.45568900 & 0.21067600 \\ \mathrm{H} & -1.86515300 & -3.21656700 & -2.37803300 \\ \mathrm{H} & -2.79553400 & -0.84302000 & -3.25648500 \\ \mathrm{H} & -4.02563400 & 0.37340700 & -1.20681400 \\ \mathrm{H} & 2.37351700 & -3.58883000 & -0.48334500 \\ \mathrm{H} & 1.04459700 & -3.68132200 & 1.84457800 \\ \mathrm{H} & 1.80935000 & -1.56683000 & 3.33312800 \\ \mathrm{H} & 3.61704800 & -0.17655200 & 1.91718500 \\ \mathrm{H} & 4.76044500 & 0.24000000 & -0.95926500 \\ \mathrm{H} & 5.84988100 & -1.89924200 & -0.81884500 \\ \mathrm{H} & 4.00939400 & -1.61914300 & -2.34335900 \\ \mathrm{H} & 2.42305100 & 2.73374000 & -2.05060500 \\ \mathrm{H} & -0.07219500 & 2.92464800 & -3.05555900 \\ \mathrm{H} & -1.72656900 & 3.56987600 & -1.04522700 \\ \mathrm{H} & 2.30027800 & 3.26337200 & 0.57468500 \\ \mathrm{H} & -1.95594200 & 3.63946100 & 2.08018200 \\ \mathrm{H} & 0.28926900 & 3.43566300 & 2.99857500 \\ \mathrm{H} & -0.47414000 & 5.53637100 & 2.10757800\end{array}$

\begin{tabular}{lrrr}
$\mathbf{A 1}^{\mathbf{C S}}$ & & & \\
$\mathrm{Ti}$ & 0.78473000 & -1.74963600 & -0.64029000 \\
$\mathrm{Ti}$ & 0.05663900 & 0.02787200 & 1.07956200 \\
$\mathrm{Si}$ & -4.60730600 & 1.00719800 & 0.41069700 \\
$\mathrm{Si}$ & 4.32515000 & -0.58152900 & -1.09657600 \\
$\mathrm{Si}$ & 2.12052700 & -0.67171500 & 4.09199800 \\
$\mathrm{C}$ & -4.01211500 & -0.64831400 & -0.22057300 \\
$\mathrm{C}$ & -3.88562600 & -1.86132100 & 0.53316100 \\
$\mathrm{C}$ & -3.47579700 & -2.90040800 & -0.33580900 \\
$\mathrm{C}$ & -3.33969300 & -2.34823100 & -1.63934200 \\
$\mathrm{C}$ & -3.67169700 & -0.97173300 & -1.57101300 \\
$\mathrm{C}$ & 3.04778400 & -1.91722900 & -1.37652800 \\
$\mathrm{C}$ & 2.77022200 & -3.03645400 & -0.52412700 \\
$\mathrm{C}$ & 1.78960700 & -3.85002600 & -1.14038200 \\
$\mathrm{C}$ & 1.44047200 & -3.24616500 & -2.37951500 \\
$\mathrm{C}$ & 2.21324900 & -2.06636500 & -2.52716100 \\
$\mathrm{C}$ & 1.14445800 & 0.58069100 & 3.10544600 \\
$\mathrm{C}$ & -0.24122800 & 0.89615800 & 3.25632200 \\
$\mathrm{C}$ & -0.57635200 & 1.94188200 & 2.35579800 \\
$\mathrm{C}$ & 0.59340000 & 2.29492900 & 1.63315200 \\
$\mathrm{C}$ & 1.64466300 & 1.46040000 & 2.08943900 \\
& & \multicolumn{2}{c}{$\mathrm{S} 28$} \\
& & &
\end{tabular}




\begin{tabular}{|c|c|c|c|}
\hline $\mathrm{H}$ & -0.72235200 & -1.85483600 & -1.66076000 \\
\hline $\mathrm{H}$ & -0.60320300 & -2.72605700 & 0.06701100 \\
\hline $\mathrm{H}$ & -1.24623900 & -1.18811000 & 1.53600300 \\
\hline $\mathrm{H}$ & 0.92972400 & -1.58807300 & 1.18563100 \\
\hline $\mathrm{H}$ & 1.49679600 & -0.18326600 & -0.02621600 \\
\hline $\mathrm{H}$ & -1.55962800 & 2.37603800 & 2.22784500 \\
\hline $\mathrm{H}$ & -0.93098900 & 0.40264200 & 3.93034800 \\
\hline $\mathrm{H}$ & 0.66161000 & 3.03783100 & 0.8477770 \\
\hline $\mathrm{H}$ & 2.66132300 & 1.47079300 & 1.71632700 \\
\hline $\mathrm{H}$ & 3.26886100 & -1.17517500 & 3.29526100 \\
\hline $\mathrm{H}$ & 2.66615700 & -0.04929000 & 5.33246900 \\
\hline $\mathrm{H}$ & 1.24513200 & -1.80149900 & 4.49751800 \\
\hline $\mathrm{H}$ & -3.27156000 & -3.92564900 & -0.05380600 \\
\hline $\mathrm{H}$ & -4.05493800 & -1.96096700 & 1.59845400 \\
\hline $\mathrm{H}$ & -3.02001000 & -2.88245000 & -2.52491500 \\
\hline $\mathrm{H}$ & -3.65138900 & -0.27604400 & -2.40199800 \\
\hline $\mathrm{H}$ & -6.09312600 & 1.09354100 & 0.32751700 \\
\hline $\mathrm{H}$ & -4.22062600 & 1.19564300 & 1.83279700 \\
\hline $\mathrm{H}$ & -4.04159500 & 2.10365300 & -0.41806800 \\
\hline $\mathrm{H}$ & 3.92343600 & 0.66138500 & -1.80633800 \\
\hline $\mathrm{H}$ & 4.49223900 & -0.30596900 & 0.35303200 \\
\hline $\mathrm{H}$ & 5.64829000 & -1.00507700 & -1.63668600 \\
\hline $\mathrm{H}$ & 3.22158200 & -3.21710500 & 0.44393100 \\
\hline $\mathrm{H}$ & 1.35767700 & -4.75296600 & -0.72744600 \\
\hline $\mathrm{H}$ & 0.70065000 & -3.61377100 & -3.07905100 \\
\hline $\mathrm{H}$ & 2.16434400 & -1.38100900 & -3.36553900 \\
\hline $\mathrm{C}$ & 1.86130200 & 4.75788000 & -1.51753500 \\
\hline $\mathrm{C}$ & 0.85447100 & 5.63583200 & -1.10676900 \\
\hline $\mathrm{C}$ & -0.48826300 & 5.28720800 & -1.27578900 \\
\hline $\mathrm{C}$ & -0.82436300 & 4.06030000 & -1.85414600 \\
\hline $\mathrm{C}$ & 0.18233100 & 3.18104700 & -2.26264400 \\
\hline $\mathrm{C}$ & 1.52533500 & 3.53065600 & -2.09540300 \\
\hline $\mathrm{H}$ & 2.90570100 & 5.03094000 & -1.39001400 \\
\hline $\mathrm{H}$ & 1.11567700 & 6.59148200 & -0.65963900 \\
\hline $\mathrm{H}$ & -1.27116200 & 5.97220400 & -0.96045900 \\
\hline $\mathrm{H}$ & -1.86864200 & 3.78816200 & -1.98460200 \\
\hline $\mathrm{H}$ & -0.07846900 & 2.22045100 & -2.69826200 \\
\hline $\mathrm{H}$ & 2.30763200 & 2.84516400 & -2.41100700 \\
\hline $\mathrm{Ti}$ & -1.71768200 & -1.29535900 & -0.23895700 \\
\hline $\mathrm{H}$ & -0.27759900 & -0.15128200 & -0.82581500 \\
\hline $\mathrm{H}$ & -1.62655200 & 0.38558300 & 0.46661600 \\
\hline
\end{tabular}

\section{$\mathrm{TS1}^{\mathrm{CS}}$}

$\mathrm{Ti}$

Ti

$\mathrm{Si}$

$\begin{array}{rrr}1.48050500 & -1.23972700 & -0.59287800 \\ -0.01213700 & 0.25186400 & 1.00816000 \\ -4.62461000 & -0.99110600 & 0.29038200\end{array}$




\begin{tabular}{|c|c|c|c|}
\hline $\mathrm{Si}$ & 4.20983900 & 1.20400100 & -1.36316100 \\
\hline $\mathrm{Si}$ & 2.19983900 & 0.49290700 & 3.99391700 \\
\hline $\mathrm{C}$ & -3.35613400 & -2.27518800 & -0.18292800 \\
\hline $\mathrm{C}$ & -2.71724700 & -3.21388600 & 0.69193400 \\
\hline $\mathrm{C}$ & -1.87987500 & -4.05635300 & -0.07310700 \\
\hline $\mathrm{C}$ & -1.98529300 & -3.65534700 & -1.43405300 \\
\hline $\mathrm{C}$ & -2.89540200 & -2.57098000 & -1.50532400 \\
\hline $\mathrm{C}$ & 3.65627600 & -0.58080400 & -1.30643100 \\
\hline $\mathrm{C}$ & 3.79624000 & -1.49673400 & -0.21236800 \\
\hline $\mathrm{C}$ & 3.31186700 & -2.76923000 & -0.60865100 \\
\hline $\mathrm{C}$ & 2.86066600 & -2.66252200 & -1.95114500 \\
\hline $\mathrm{C}$ & 3.07004600 & -1.32655400 & -2.37488200 \\
\hline $\mathrm{C}$ & 0.75977600 & 1.20188900 & 3.03356200 \\
\hline $\mathrm{C}$ & -0.62514600 & 0.88393000 & 3.20459200 \\
\hline $\mathrm{C}$ & -1.39414000 & 1.69214100 & 2.32815100 \\
\hline $\mathrm{C}$ & -0.49960300 & 2.52702000 & 1.60672300 \\
\hline $\mathrm{C}$ & 0.81521400 & 2.22969400 & 2.03553900 \\
\hline $\mathrm{H}$ & 0.24947800 & -2.12736200 & -1.53318000 \\
\hline $\mathrm{H}$ & 0.65509900 & -2.56651900 & 0.27751700 \\
\hline $\mathrm{H}$ & -0.71713200 & -1.29943000 & 1.55197000 \\
\hline $\mathrm{H}$ & 1.52006800 & -0.72147000 & 1.15701900 \\
\hline $\mathrm{H}$ & 1.13305700 & 0.55646900 & -0.41337100 \\
\hline $\mathrm{H}$ & -2.46994400 & 1.66041200 & 2.21353500 \\
\hline $\mathrm{H}$ & -1.02224700 & 0.13672400 & 3.88041500 \\
\hline $\mathrm{H}$ & -0.77243400 & 3.22960100 & 0.82789500 \\
\hline $\mathrm{H}$ & 1.72072600 & 2.68169100 & 1.64848500 \\
\hline $\mathrm{H}$ & 3.44812000 & 0.60723800 & 3.19570800 \\
\hline $\mathrm{H}$ & 2.39966600 & 1.24328800 & 5.26612300 \\
\hline $\mathrm{H}$ & 1.94466200 & -0.92966200 & 4.33717400 \\
\hline $\mathrm{H}$ & -1.24227700 & -4.84236100 & 0.31250800 \\
\hline $\mathrm{H}$ & -2.83379200 & -3.25074100 & 1.76854700 \\
\hline $\mathrm{H}$ & -1.44928400 & -4.09066400 & -2.26800500 \\
\hline $\mathrm{H}$ & -3.18543200 & -2.04872100 & -2.40754800 \\
\hline $\mathrm{H}$ & -6.00110800 & -1.56343200 & 0.24623000 \\
\hline $\mathrm{H}$ & -4.38910500 & -0.50434300 & 1.67584900 \\
\hline $\mathrm{H}$ & -4.58574000 & 0.15466300 & -0.65600600 \\
\hline $\mathrm{H}$ & 3.38307000 & 1.95641900 & -2.34333300 \\
\hline $\mathrm{H}$ & 4.09891200 & 1.82968600 & -0.02066100 \\
\hline $\mathrm{H}$ & 5.63220400 & 1.29950100 & -1.79697900 \\
\hline $\mathrm{H}$ & 4.19829000 & -1.25304100 & 0.76350300 \\
\hline $\mathrm{H}$ & 3.27175100 & -3.65750600 & 0.00872300 \\
\hline $\mathrm{H}$ & 2.41045000 & -3.45323100 & -2.53723200 \\
\hline $\mathrm{H}$ & 2.79815100 & -0.92739000 & -3.34641700 \\
\hline $\mathrm{C}$ & -0.40089900 & 4.93182200 & -1.78154900 \\
\hline $\mathrm{C}$ & -1.67471000 & 5.27827400 & -1.32194300 \\
\hline $\mathrm{C}$ & -2.70059500 & 4.32871900 & -1.32379700 \\
\hline
\end{tabular}




$\begin{array}{cccc}\mathrm{C} & -2.45173700 & 3.03197700 & -1.78147400 \\ \mathrm{C} & -1.17715200 & 2.68377900 & -2.23894100 \\ \mathrm{C} & -0.15260900 & 3.63532000 & -2.24074200 \\ \mathrm{H} & 0.39504900 & 5.67220300 & -1.78562200 \\ \mathrm{H} & -1.86883600 & 6.28755500 & -0.96805600 \\ \mathrm{H} & -3.69260300 & 4.60030700 & -0.97180200 \\ \mathrm{H} & -3.24529800 & 2.28949400 & -1.77826200 \\ \mathrm{H} & -0.98367300 & 1.66977300 & -2.57733700 \\ \mathrm{H} & 0.83821000 & 3.36448000 & -2.59661800 \\ \mathrm{Ti} & -1.02229800 & -1.82802300 & -0.23951700 \\ \mathrm{H} & -1.00728500 & -0.70278200 & -1.53292600 \\ \mathrm{H} & -1.59252000 & -0.13441800 & 0.26038600\end{array}$

$\mathrm{A} 2^{\mathrm{CS}}$

$\mathrm{Ti}$

1.15797300

$-1.53641200$

$-0.57491900$

0.01857000

0.30626500

0.94398400

$-4.82854900$

0.16503400

0.26498000

4.59618200

$-0.10320600$

$-0.86787500$

1.63239400

$-0.23831400$

4.26051800

$-3.87919000$

$-1.34774300$

$-0.27444800$

$-3.36201500$

$-2.38544200$

0.56888300

$-2.75529700$

$-3.37175700$

$-0.23706500$

$-2.88511700$

$-2.96774100$

$-1.59546200$

$-3.58799300$

$-1.73774900$

$-1.62204400$

3.48828400

$-1.59814500$

$-1.05287900$

3.21097200

$-2.59850500$

$-0.06592600$

2.38005200

$-3.59527300$

$-0.63909600$

2.12679500

$-3.23140100$

$-1.98746200$

2.80015900

$-2.01107700$

$-2.23727500$

0.70195900

0.92452200

3.12824600

$-0.71711500$

1.10060300

3.05298000

$-1.00411000$

2.12196900

2.11301600

0.23209500

2.59985300

1.59873000

1.27463400

1.87360700

2.22012600

$-0.15784700$

$-1.49345700$

$-1.78462700$

$-0.18817600$

$-2.59033900$

$-0.09929500$

$-1.21432100$

$-0.91597100$

1.29419600

1.15645200

$-1.09908400$

1.19645900

1.27264800

0.28337000

$-0.40719200$

$-1.98955900$

2.46231600

1.82298100

$-1.45371000$

0.53403800

3.60900000

0.35047100

3.35223700

0.82744300

2.33231800

1.98866400

2.01458500

2.98887400

$-0.49617800$

3.71162900

$\mathrm{H}$

1.78832900

0.36453700

5.61425800

0.89598500

$-1.51963800$

4.41499700 


$\begin{array}{lrrc}\mathrm{H} & -2.24812800 & -4.25976600 & 0.11796900 \\ \mathrm{H} & -3.40061800 & -2.39448300 & 1.65118400 \\ \mathrm{H} & -2.49660400 & -3.49675800 & -2.45719100 \\ \mathrm{H} & -3.86255900 & -1.18771700 & -2.51157300 \\ \mathrm{H} & -6.29277400 & -0.11185300 & 0.32290600 \\ \mathrm{H} & -4.40032100 & 0.58683800 & 1.62565400 \\ \mathrm{H} & -4.61933100 & 1.28213300 & -0.69302200 \\ \mathrm{H} & 4.17638000 & 0.95311300 & -1.82557800 \\ \mathrm{H} & 4.53950300 & 0.41958500 & 0.52148500 \\ \mathrm{H} & 6.01227800 & -0.45707200 & -1.16726200 \\ \mathrm{H} & 3.56946500 & -2.58960500 & 0.95643600 \\ \mathrm{H} & 1.98787500 & -4.46759200 & -0.13109000 \\ \mathrm{H} & 1.50052900 & -3.76878200 & -2.68782700 \\ \mathrm{H} & 2.76900000 & -1.45958100 & -3.17096900 \\ \mathrm{C} & 1.31278200 & 4.67203000 & -1.80303700 \\ \mathrm{C} & 0.26344200 & 5.49560000 & -1.38527700 \\ \mathrm{C} & -1.05047400 & 5.01840100 & -1.40711600 \\ \mathrm{C} & -1.31387200 & 3.71700600 & -1.84242200 \\ \mathrm{C} & -0.26497900 & 2.89045300 & -2.25722500 \\ \mathrm{C} & 1.04830000 & 3.37075500 & -2.23955100 \\ \mathrm{H} & 2.33373700 & 5.04537600 & -1.79246000 \\ \mathrm{H} & 0.46836800 & 6.50889000 & -1.04924500 \\ \mathrm{H} & -1.86697000 & 5.66171000 & -1.08879700 \\ \mathrm{H} & -2.33337800 & 3.34055900 & -1.85657900 \\ \mathrm{H} & -0.47349800 & 1.87310400 & -2.57600500 \\ \mathrm{Ti} & 1.86461400 & 2.72894100 & -2.56086100 \\ \mathrm{H} & -1.52313500 & -1.33356700 & -0.51873700 \\ \mathrm{H} & -1.68854000 & -0.32025400 & -1.84980300 \\ & -1.41173200 & 0.47541200 & -0.11993600\end{array}$

\section{TS2 $^{\mathrm{CS}}$}

Ti

$\mathrm{Ti}$

$\mathrm{Si}$

$\mathrm{Si}$

$\mathrm{Si}$

C

C

C

C

C

C

C

C

C

C

$\begin{array}{ccc}-0.73630600 & -1.75587000 & -0.37728000 \\ 0.73906200 & 0.15620400 & 1.00700300 \\ -3.45873600 & 3.38174900 & -1.54346100 \\ 1.15344700 & -2.59252600 & -3.47849700 \\ 1.44069700 & -2.32979700 & 3.66048200 \\ -3.50004500 & 2.23393900 & -0.07004700 \\ -2.92169900 & 2.46594400 & 1.21792700 \\ -3.22114600 & 1.36837600 & 2.06669200 \\ -3.97778700 & 0.43526300 & 1.31883900 \\ -4.13862900 & 0.95547000 & 0.00813600 \\ -0.02497600 & -3.16005900 & -2.14719300 \\ 0.32695100 & -3.82605000 & -0.93175000 \\ -0.85293700 & -4.15076000 & -0.21966900 \\ -1.96497800 & -3.69157000 & -0.97057200 \\ -1.45843900 & -3.07919000 & -2.15058400\end{array}$




\begin{tabular}{|c|c|c|c|}
\hline $\mathrm{C}$ & 1.86995300 & -0.68755000 & 2.88476800 \\
\hline $\mathrm{C}$ & 1.33233800 & 0.59654700 & 3.22235600 \\
\hline $\mathrm{C}$ & 1.97143900 & 1.58335100 & 2.42745300 \\
\hline $\mathrm{C}$ & 2.93049500 & 0.92774300 & 1.60507600 \\
\hline $\mathrm{C}$ & 2.87122900 & -0.45351500 & 1.87873100 \\
\hline $\mathrm{H}$ & -2.07694800 & -1.24545200 & 0.68143000 \\
\hline $\mathrm{H}$ & -0.83261600 & 0.17442500 & 1.79928800 \\
\hline $\mathrm{H}$ & -0.28154800 & 1.62294100 & 0.57866900 \\
\hline $\mathrm{H}$ & 0.85021100 & -1.56484200 & 0.46265200 \\
\hline $\mathrm{H}$ & -0.14446400 & -0.04881400 & -0.57729500 \\
\hline $\mathrm{H}$ & 1.75734700 & 2.64451800 & 2.43338500 \\
\hline $\mathrm{H}$ & 0.55207200 & 0.78297400 & 3.94900800 \\
\hline $\mathrm{H}$ & 3.54769800 & 1.40201600 & 0.85100400 \\
\hline $\mathrm{H}$ & 3.45736100 & -1.22002000 & 1.38709100 \\
\hline $\mathrm{H}$ & 1.82690100 & -3.43509000 & 2.74438700 \\
\hline $\mathrm{H}$ & 2.17758000 & -2.52188500 & 4.94248900 \\
\hline $\mathrm{H}$ & -0.01296700 & -2.41292500 & 3.96093700 \\
\hline $\mathrm{H}$ & -2.89836100 & 1.25039100 & 3.09304000 \\
\hline $\mathrm{H}$ & -2.33169800 & 3.33097600 & 1.49494400 \\
\hline $\mathrm{H}$ & -4.33550900 & -0.52325200 & 1.67118900 \\
\hline $\mathrm{H}$ & -4.64711700 & 0.45615700 & -0.80781000 \\
\hline $\mathrm{H}$ & -4.62507600 & 4.31423200 & -1.50610800 \\
\hline $\mathrm{H}$ & -2.22711700 & 4.21203200 & -1.51865400 \\
\hline $\mathrm{H}$ & -3.54360000 & 2.60882300 & -2.80819300 \\
\hline $\mathrm{H}$ & 0.70698400 & -1.30370800 & -4.06932600 \\
\hline $\mathrm{H}$ & 2.51282800 & -2.43407200 & -2.89740900 \\
\hline $\mathrm{H}$ & 1.23805700 & -3.58843200 & -4.58481300 \\
\hline $\mathrm{H}$ & 1.33697700 & -4.02314700 & -0.59455200 \\
\hline $\mathrm{H}$ & -0.89452400 & -4.62272100 & 0.75570600 \\
\hline $\mathrm{H}$ & -3.00682600 & -3.77315000 & -0.68912000 \\
\hline $\mathrm{H}$ & -2.06250900 & -2.62612000 & -2.92708300 \\
\hline $\mathrm{C}$ & 4.40896400 & 1.89219000 & -1.89297600 \\
\hline $\mathrm{C}$ & 4.40619400 & 3.15777500 & -1.29788500 \\
\hline $\mathrm{C}$ & 3.19499200 & 3.79495000 & -1.01290900 \\
\hline $\mathrm{C}$ & 1.98530600 & 3.16669200 & -1.32194800 \\
\hline $\mathrm{C}$ & 1.98635400 & 1.90050100 & -1.91870800 \\
\hline $\mathrm{C}$ & 3.20000000 & 1.26385400 & -2.20284800 \\
\hline $\mathrm{H}$ & 5.35162300 & 1.40072700 & -2.11987700 \\
\hline $\mathrm{H}$ & 5.34693700 & 3.64898700 & -1.06251900 \\
\hline $\mathrm{H}$ & 3.19478000 & 4.78174800 & -0.55688400 \\
\hline $\mathrm{H}$ & 1.04001600 & 3.65567000 & -1.10427800 \\
\hline $\mathrm{H}$ & 1.04321400 & 1.42262800 & -2.16794100 \\
\hline $\mathrm{H}$ & 3.19960200 & 0.28169600 & -2.66792800 \\
\hline $\mathrm{Ti}$ & -1.83128600 & 0.59659200 & 0.29053600 \\
\hline $\mathrm{H}$ & -1.93089500 & -0.61411100 & -1.15077800 \\
\hline $\mathrm{H}$ & -1.49793500 & 1.43905800 & -1.13701000 \\
\hline
\end{tabular}




\begin{tabular}{|c|c|c|c|}
\hline $\mathrm{A3}^{\mathrm{CS}}$ & & & \\
\hline $\mathrm{Ti}$ & -0.04833500 & 1.69288900 & -0.58669000 \\
\hline $\mathrm{Ti}$ & -0.55887900 & -0.59519600 & 0.83456500 \\
\hline $\mathrm{Si}$ & 4.67399200 & -1.77762600 & -1.67608200 \\
\hline $\mathrm{Si}$ & -2.58232000 & 2.45782000 & -3.12828000 \\
\hline $\mathrm{Si}$ & -1.95753300 & 1.72183200 & 3.46479000 \\
\hline $\mathrm{C}$ & 4.20209700 & -0.70467200 & -0.22084600 \\
\hline $\mathrm{C}$ & 3.77380000 & -1.14669300 & 1.07133900 \\
\hline $\mathrm{C}$ & 3.55738900 & -0.01658800 & 1.90136700 \\
\hline $\mathrm{C}$ & 3.83774000 & 1.14304000 & 1.13749800 \\
\hline $\mathrm{C}$ & 4.22341400 & 0.72534300 & -0.16190600 \\
\hline $\mathrm{C}$ & -1.46557700 & 3.12010000 & -1.78771400 \\
\hline $\mathrm{C}$ & -1.81556200 & 3.29851200 & -0.40936400 \\
\hline $\mathrm{C}$ & -0.71322700 & 3.84350700 & 0.28264600 \\
\hline $\mathrm{C}$ & 0.34811700 & 4.02085200 & -0.64727600 \\
\hline $\mathrm{C}$ & -0.10886400 & 3.57788600 & -1.91632200 \\
\hline $\mathrm{C}$ & -1.78757600 & 0.00937700 & 2.75547400 \\
\hline $\mathrm{C}$ & -0.80999900 & -0.97937900 & 3.11409600 \\
\hline $\mathrm{C}$ & -1.12346300 & -2.19847200 & 2.45670800 \\
\hline $\mathrm{C}$ & -2.30360100 & -1.98775900 & 1.69423500 \\
\hline $\mathrm{C}$ & -2.70640400 & -0.64721100 & 1.87051500 \\
\hline $\mathrm{H}$ & 1.32866000 & 1.73238400 & 0.53062600 \\
\hline $\mathrm{H}$ & 0.90694400 & 0.02156600 & 1.59555100 \\
\hline $\mathrm{H}$ & 1.00808300 & -1.54061700 & 0.45133600 \\
\hline $\mathrm{H}$ & -1.46292200 & 0.61525400 & -0.19180500 \\
\hline $\mathrm{H}$ & 0.33044800 & -0.09403000 & -0.68992700 \\
\hline $\mathrm{H}$ & -0.55393700 & -3.11758000 & 2.51047400 \\
\hline $\mathrm{H}$ & 0.02849700 & -0.82268900 & 3.78006400 \\
\hline $\mathrm{H}$ & -2.76884300 & -2.70962600 & 1.03426200 \\
\hline $\mathrm{H}$ & -3.55376600 & -0.17748300 & 1.38646100 \\
\hline $\mathrm{H}$ & -2.77830400 & 2.57162500 & 2.56241400 \\
\hline $\mathrm{H}$ & -2.65318200 & 1.67836100 & 4.78462600 \\
\hline $\mathrm{H}$ & -0.63240300 & 2.35805900 & 3.68827300 \\
\hline $\mathrm{H}$ & 3.20792600 & -0.03571700 & 2.92560600 \\
\hline $\mathrm{H}$ & 3.61573400 & -2.17809100 & 1.36165400 \\
\hline $\mathrm{H}$ & 3.73775000 & 2.16680000 & 1.47399300 \\
\hline $\mathrm{H}$ & 4.46910600 & 1.38383500 & -0.98624800 \\
\hline $\mathrm{H}$ & 6.13036600 & -2.10644500 & -1.62483700 \\
\hline $\mathrm{H}$ & 3.92785800 & -3.06126300 & -1.63565500 \\
\hline $\mathrm{H}$ & 4.42126000 & -1.06370700 & -2.95292700 \\
\hline $\mathrm{H}$ & -1.88819600 & 1.41748300 & -3.93708500 \\
\hline $\mathrm{H}$ & -3.79294500 & 1.86527500 & -2.50219000 \\
\hline $\mathrm{H}$ & -3.00873800 & 3.53572300 & -4.06374700 \\
\hline $\mathrm{H}$ & -2.76038500 & 3.01902400 & 0.03805000 \\
\hline $\mathrm{H}$ & -0.66622300 & 4.04461100 & 1.34541800 \\
\hline
\end{tabular}




$\begin{array}{lrrr}\mathrm{H} & 1.33707800 & 4.39811700 & -0.42185800 \\ \mathrm{H} & 0.47654800 & 3.58065300 & -2.82843400 \\ \mathrm{C} & -3.40002300 & -3.24257500 & -1.77098900 \\ \mathrm{C} & -3.08330400 & -4.46824600 & -1.17741100 \\ \mathrm{C} & -1.75686200 & -4.77020000 & -0.85349400 \\ \mathrm{C} & -0.74487900 & -3.84604100 & -1.12497500 \\ \mathrm{C} & -1.05855600 & -2.61873000 & -1.72311400 \\ \mathrm{C} & -2.38851900 & -2.31771300 & -2.04389800 \\ \mathrm{H} & -4.43114700 & -3.01242800 & -2.02612200 \\ \mathrm{H} & -3.86954100 & -5.19021100 & -0.97184900 \\ \mathrm{H} & -1.51339100 & -5.72608300 & -0.39701300 \\ \mathrm{H} & 0.28884400 & -4.07360900 & -0.88091200 \\ \mathrm{H} & -0.26161200 & -1.92046400 & -1.96395700 \\ \mathrm{H} & -2.62974500 & -1.36664900 & -2.51063100 \\ \mathrm{Ti} & 1.99141100 & 0.04123400 & 0.10868000 \\ \mathrm{H} & 1.58097400 & 1.23300600 & -1.33865300 \\ \mathrm{H} & 2.04209900 & -0.87587400 & -1.31457400\end{array}$

\begin{tabular}{|c|c|c|c|}
\hline \multicolumn{4}{|c|}{$\mathrm{TS3}^{\mathrm{CS}}$} \\
\hline $\mathrm{Ti}$ & -0.39692000 & -0.91734200 & -0.76152600 \\
\hline $\mathrm{Ti}$ & -1.14265600 & 0.80713200 & 1.06528300 \\
\hline $\mathrm{Si}$ & 4.62918900 & 1.87302900 & 0.25219000 \\
\hline $\mathrm{Si}$ & -2.61741600 & -3.84217000 & 0.01302300 \\
\hline $\mathrm{Si}$ & -4.18974300 & 2.34077700 & -0.55852000 \\
\hline $\mathrm{C}$ & 3.07429400 & 2.48150300 & -0.57945900 \\
\hline $\mathrm{C}$ & 2.16554100 & 3.46570800 & -0.07977900 \\
\hline $\mathrm{C}$ & 1.17879400 & 3.73187100 & -1.06475800 \\
\hline $\mathrm{C}$ & 1.44346800 & 2.90221100 & -2.18346300 \\
\hline $\mathrm{C}$ & 2.60228400 & 2.13670700 & -1.88604800 \\
\hline $\mathrm{C}$ & -1.85835500 & -2.70452100 & -1.25132000 \\
\hline $\mathrm{C}$ & -2.42360100 & -1.46888800 & -1.72626700 \\
\hline $\mathrm{C}$ & -1.59982700 & -0.96309000 & -2.77059700 \\
\hline $\mathrm{C}$ & -0.52377000 & -1.87098200 & -2.95676400 \\
\hline $\mathrm{C}$ & -0.68236100 & -2.92889600 & -2.03386100 \\
\hline $\mathrm{C}$ & -3.36907000 & 1.69356800 & 0.99247600 \\
\hline $\mathrm{C}$ & -2.61177500 & 2.46760600 & 1.93036400 \\
\hline $\mathrm{C}$ & -2.21729800 & 1.63516000 & 3.00415400 \\
\hline $\mathrm{C}$ & -2.72309500 & 0.32974200 & 2.74706300 \\
\hline $\mathrm{C}$ & -3.44060600 & 0.37055600 & 1.52072700 \\
\hline $\mathrm{H}$ & -0.83733500 & 0.83226800 & -0.85396000 \\
\hline $\mathrm{H}$ & -0.58233800 & 2.30499400 & 0.20813000 \\
\hline $\mathrm{H}$ & 0.47685200 & 1.56856400 & 1.52793500 \\
\hline $\mathrm{H}$ & -0.96921700 & -1.03128200 & 0.98593300 \\
\hline $\mathrm{H}$ & 0.91577700 & -0.04229100 & 0.57743000 \\
\hline $\mathrm{H}$ & -1.61158400 & 1.93436000 & 3.85094200 \\
\hline $\mathrm{H}$ & -2.35251600 & 3.51315500 & 1.81829400 \\
\hline
\end{tabular}




$\begin{array}{lrrr}\mathrm{H} & -2.58115800 & -0.54619300 & 3.36834100 \\ \mathrm{H} & -3.93298700 & -0.47381900 & 1.05702600 \\ \mathrm{H} & -4.37905000 & 1.24148400 & -1.54019900 \\ \mathrm{H} & -5.53069100 & 2.90444300 & -0.23823800 \\ \mathrm{H} & -3.37021300 & 3.42116400 & -1.16567700 \\ \mathrm{H} & 0.35221400 & 4.42371300 & -0.96518500 \\ \mathrm{H} & 2.21183300 & 3.92319300 & 0.90133900 \\ \mathrm{H} & 0.85585700 & 2.84402200 & -3.09120900 \\ \mathrm{H} & 3.04840200 & 1.39581400 & -2.53786800 \\ \mathrm{H} & 5.77606300 & 2.77850300 & -0.05369900 \\ \mathrm{H} & 4.46180000 & 1.84978500 & 1.72846800 \\ \mathrm{H} & 4.98538400 & 0.51957800 & -0.24486100 \\ \mathrm{H} & -1.56626000 & -4.55697100 & 0.78738000 \\ \mathrm{H} & -3.47911600 & -3.07218900 & 0.94838900 \\ \mathrm{H} & -3.47047700 & -4.88031500 & -0.63325800 \\ \mathrm{H} & -3.34241100 & -1.01776200 & -1.37951200 \\ \mathrm{H} & -1.75486100 & -0.04132100 & -3.31550200 \\ \mathrm{H} & 0.30016300 & -1.74847800 & -3.64875200 \\ \mathrm{H} & -0.00021000 & -3.76402700 & -1.91809000 \\ \mathrm{C} & 1.91794800 & -2.76879100 & 2.10913300 \\ \mathrm{C} & 3.19647900 & -2.30672200 & 2.42441400 \\ \mathrm{C} & 4.20538000 & -2.32936000 & 1.45667500 \\ \mathrm{C} & 3.93693500 & -2.80991200 & 0.17363000 \\ \mathrm{C} & 2.65766900 & -3.27425200 & -0.14334100 \\ \mathrm{C} & 1.64774100 & -3.25627400 & 0.82499800 \\ \mathrm{H} & 1.13071500 & -2.75579500 & 2.85806000 \\ \mathrm{H} & 3.40918800 & -1.93143700 & 3.42182600 \\ \mathrm{H} & 5.20132200 & -1.97205500 & 1.70391400 \\ \mathrm{H} & 4.72255500 & -2.82576700 & -0.57682900 \\ \mathrm{H} & 2.45236800 & -3.65995800 & -1.13862500 \\ \mathrm{H} & 0.66062200 & -3.64943800 & 0.59742900 \\ \mathrm{H} & 0.94277400 & 1.46134000 & -0.35536600 \\ \mathrm{H} & 1.05042700 & -0.00091100 & -1.45629300 \\ & 1.90945100 & 0.38954100 & 0.61076600 \\ & & & \\ \mathrm{H} & & \end{array}$

$\begin{array}{cccc}\mathbf{A 4}^{\mathbf{C S}} & & & \\ \mathrm{Ti} & -0.74194800 & 0.96012300 & 0.80571300 \\ \mathrm{Ti} & -0.93505700 & -0.92350500 & -0.98024400 \\ \mathrm{Si} & 3.75519600 & -3.22785200 & -0.69751900 \\ \mathrm{Si} & -2.65278100 & 3.81737300 & -0.48820700 \\ \mathrm{Si} & -4.20166500 & -2.41429400 & 0.20567600 \\ \mathrm{C} & 2.83530200 & -2.65197700 & 0.81892000 \\ \mathrm{C} & 1.66811200 & -3.24973400 & 1.39918300 \\ \mathrm{C} & 1.31988600 & -2.54744400 & 2.58318800 \\ \mathrm{C} & 2.27176600 & -1.51135900 & 2.76222700 \\ \mathrm{C} & 3.19324600 & -1.57309800 & 1.69009200\end{array}$




\begin{tabular}{|c|c|c|c|}
\hline $\mathrm{C}$ & -2.23059900 & 2.72916400 & 0.96262600 \\
\hline $\mathrm{C}$ & -2.93283400 & 1.53904800 & 1.36605800 \\
\hline $\mathrm{C}$ & -2.36052900 & 1.06280000 & 2.57341200 \\
\hline $\mathrm{C}$ & -1.31234100 & 1.94003200 & 2.94489800 \\
\hline $\mathrm{C}$ & -1.22171800 & 2.95365300 & 1.96212800 \\
\hline $\mathrm{C}$ & -3.07206900 & -2.01109400 & -1.22548600 \\
\hline $\mathrm{C}$ & -2.05546400 & -2.85284200 & -1.78198200 \\
\hline $\mathrm{C}$ & -1.46998000 & -2.19820900 & -2.89655200 \\
\hline $\mathrm{C}$ & -2.12199200 & -0.94255500 & -3.04488700 \\
\hline $\mathrm{C}$ & -3.10274100 & -0.83165500 & -2.02703700 \\
\hline $\mathrm{H}$ & -0.84748100 & -0.84133800 & 0.91041600 \\
\hline $\mathrm{H}$ & -0.11588800 & -2.25781900 & -0.03654600 \\
\hline $\mathrm{H}$ & 0.91433000 & -1.09716300 & -1.14068500 \\
\hline $\mathrm{H}$ & -0.94131200 & 0.91193200 & -1.01963000 \\
\hline $\mathrm{H}$ & 1.97718600 & 0.54303700 & 0.12457700 \\
\hline $\mathrm{H}$ & -0.66864500 & -2.58396800 & -3.51439800 \\
\hline $\mathrm{H}$ & -1.77206000 & -3.82938700 & -1.40893000 \\
\hline $\mathrm{H}$ & -1.89268000 & -0.18743400 & -3.78812900 \\
\hline $\mathrm{H}$ & -3.74476500 & 0.02489800 & -1.86557000 \\
\hline $\mathrm{H}$ & -4.83784800 & -1.16855500 & 0.70899200 \\
\hline $\mathrm{H}$ & -5.28735800 & -3.34173500 & -0.22141100 \\
\hline $\mathrm{H}$ & -3.45641000 & -3.07441900 & 1.30972300 \\
\hline $\mathrm{H}$ & 0.47312900 & -2.75574600 & 3.22487000 \\
\hline $\mathrm{H}$ & 1.13541700 & -4.10249100 & 0.99787400 \\
\hline $\mathrm{H}$ & 2.27215600 & -0.77769800 & 3.55807000 \\
\hline $\mathrm{H}$ & 4.02571900 & -0.89493600 & 1.54063900 \\
\hline $\mathrm{H}$ & 4.81461900 & -4.21799000 & -0.34590600 \\
\hline $\mathrm{H}$ & 2.82337100 & -3.88566000 & -1.65006100 \\
\hline $\mathrm{H}$ & 4.42325700 & -2.07544500 & -1.35939300 \\
\hline $\mathrm{H}$ & -1.41880300 & 4.39576800 & -1.08971000 \\
\hline $\mathrm{H}$ & -3.38268100 & 3.03359200 & -1.51916600 \\
\hline $\mathrm{H}$ & -3.51940800 & 4.96231100 & -0.08598200 \\
\hline $\mathrm{H}$ & -3.76877900 & 1.09041500 & 0.84725000 \\
\hline $\mathrm{H}$ & -2.64977300 & 0.16179400 & 3.10018300 \\
\hline $\mathrm{H}$ & -0.65710600 & 1.82168800 & 3.79889100 \\
\hline $\mathrm{H}$ & -0.50191400 & 3.76508200 & 1.96487300 \\
\hline $\mathrm{C}$ & 3.12795500 & 3.63795300 & 0.01950600 \\
\hline $\mathrm{C}$ & 2.00673900 & 3.61353600 & -0.81630000 \\
\hline $\mathrm{C}$ & 2.06899200 & 2.96195200 & -2.05194000 \\
\hline $\mathrm{C}$ & 3.25294800 & 2.33696700 & -2.45238000 \\
\hline $\mathrm{C}$ & 4.37370700 & 2.36308500 & -1.61798900 \\
\hline $\mathrm{C}$ & 4.31120900 & 3.01270000 & -0.38165100 \\
\hline $\mathrm{H}$ & 3.08171700 & 4.15086400 & 0.97682600 \\
\hline $\mathrm{H}$ & 1.08963000 & 4.11323300 & -0.51436300 \\
\hline $\mathrm{H}$ & 1.19688700 & 2.94506700 & -2.69988600 \\
\hline $\mathrm{H}$ & 3.30375600 & 1.83396300 & -3.41442000 \\
\hline
\end{tabular}




$\begin{array}{cccc}\mathrm{H} & 5.29689500 & 1.88414400 & -1.93394000 \\ \mathrm{H} & 5.18582700 & 3.03931800 & 0.26327500 \\ \mathrm{Ti} & 1.06070000 & -1.08775800 & 0.71525100 \\ \mathrm{H} & 0.76643000 & 0.41214100 & 1.69471100 \\ \mathrm{H} & 2.33323900 & -0.01110400 & -0.30436600\end{array}$

\section{$\mathrm{TS}^{\mathrm{CS}}$}

$\mathrm{Ti}$

Ti

$\mathrm{Si}$

$\mathrm{Si}$

$\mathrm{Si}$

C

C

C

C

C

C

C

C

C

C

C

$\begin{array}{ccc}-0.88757900 & -0.78193700 & -1.10089800 \\ -0.76056400 & 0.47784600 & 1.19208200 \\ 3.14773400 & 3.84133300 & 0.28957400 \\ -1.72986900 & -4.29085400 & -0.62683000 \\ -4.44684400 & 1.21054400 & 1.50788500 \\ 1.89415300 & 3.40151200 & -1.02074300 \\ 0.53591800 & 3.86000700 & -1.10012000 \\ -0.05686800 & 3.36538600 & -2.29150000 \\ 0.91703800 & 2.59719300 & -2.97358200 \\ 2.10342600 & 2.61362000 & -2.19868600 \\ -2.02202400 & -2.72301900 & -1.58785900 \\ -3.02906500 & -1.73332300 & -1.31617300 \\ -2.97472900 & -0.74016800 & -2.32365400 \\ -1.95648300 & -1.09135400 & -3.24299700 \\ -1.36125300 & -2.29703900 & -2.79596700 \\ -2.82502000 & 0.85330200 & 2.36038400 \\ -1.85289000 & 1.80980400 & 2.80228400 \\ -0.79683600 & 1.12876600 & 3.46442500 \\ -1.10553800 & -0.25779800 & 3.44050000 \\ -2.34296700 & -0.42504100 & 2.76934400 \\ -1.29415100 & 0.90032300 & -0.62935700 \\ -0.53781800 & 2.17077800 & 0.51248500 \\ 1.00746400 & 0.97313400 & 0.84784900 \\ -0.58210900 & -1.25988300 & 0.64781400 \\ 2.90383200 & -0.26998800 & -0.93862500 \\ 0.08315100 & 1.58461600 & 3.90054500 \\ -1.91186900 & 2.88142500 & 2.65653000 \\ -0.48709900 & -1.05622600 & 3.83492900 \\ -2.82415300 & -1.37391100 & 2.56768700 \\ -4.95487600 & -0.02566200 & 0.85795600 \\ -5.47247600 & 1.66742100 & 2.48828300 \\ -4.29208500 & 2.28394900 & 0.49066100 \\ -1.08052400 & 3.52446700 & -2.60736900 \\ 0.04179300 & 4.48607400 & -0.36781100 \\ 0.76984500 & 2.05542100 & -3.89889800 \\ 3.01718000 & 2.08519000 & -2.44680900 \\ 3.92170300 & 5.05812000 & -0.09109200 \\ 2.46078500 & 4.12465700 & 1.57654600 \\ 4.11865800 & 2.72959500 & 0.46732100\end{array}$




$\begin{array}{cccc}\mathrm{H} & -0.27396900 & -4.59815100 & -0.55249700 \\ \mathrm{H} & -2.27194000 & -4.14712200 & 0.75007500 \\ \mathrm{H} & -2.39322000 & -5.46481600 & -1.26311200 \\ \mathrm{H} & -3.70762100 & -1.73573200 & -0.47358500 \\ \mathrm{H} & -3.58037500 & 0.15681300 & -2.36183700 \\ \mathrm{H} & -1.65019000 & -0.51148500 & -4.10473400 \\ \mathrm{H} & -0.54540800 & -2.81498900 & -3.28839900 \\ \mathrm{C} & 3.77338800 & -3.53110200 & -0.11467200 \\ \mathrm{C} & 2.83173100 & -3.24770200 & 0.87830000 \\ \mathrm{C} & 3.17283400 & -2.41164300 & 1.94456000 \\ \mathrm{C} & 4.45392000 & -1.85899800 & 2.01859400 \\ \mathrm{C} & 5.39501500 & -2.14292100 & 1.02553400 \\ \mathrm{C} & 5.05480200 & -2.97902600 & -0.04101300 \\ \mathrm{H} & 3.51094500 & -4.18742800 & -0.94072800 \\ \mathrm{H} & 1.83567200 & -3.67913300 & 0.82285500 \\ \mathrm{H} & 2.44069500 & -2.19256700 & 2.71764200 \\ \mathrm{H} & 4.72023900 & -1.21231300 & 2.85073100 \\ \mathrm{H} & 6.39394700 & -1.71861200 & 1.08636300 \\ \mathrm{H} & 5.78913800 & -3.20487300 & -0.81000900 \\ \mathrm{Ti} & 0.49114300 & 1.52414200 & -0.84524800 \\ \mathrm{H} & 0.58721900 & 0.01452600 & -1.86643500 \\ \mathrm{H} & 3.26602100 & 0.11817900 & -0.41613600\end{array}$

$\begin{array}{cccc}\mathbf{A 5}^{\mathbf{C S}} & & & \\ \mathrm{Ti} & -0.85260100 & -0.25132700 & -0.57297600 \\ \mathrm{Ti} & 0.94231400 & 1.22866200 & 0.88005900 \\ \mathrm{Si} & 4.57032700 & -2.59607200 & 1.25945300 \\ \mathrm{Si} & -3.70263100 & 2.09816500 & -1.21567300 \\ \mathrm{Si} & 1.27401400 & 4.36811500 & -1.10890100 \\ \mathrm{C} & 3.50690300 & -2.37737300 & -0.25783400 \\ \mathrm{C} & 3.66650500 & -1.38993900 & -1.28662700 \\ \mathrm{C} & 2.69945000 & -1.61262000 & -2.29872900 \\ \mathrm{C} & 1.92422600 & -2.73601000 & -1.92607900 \\ \mathrm{C} & 2.41398400 & -3.20439600 & -0.67875900 \\ \mathrm{C} & -2.69735500 & 0.63291900 & -1.78442600 \\ \mathrm{C} & -1.52054400 & 0.68265600 & -2.60186300 \\ \mathrm{C} & -1.08983600 & -0.64427200 & -2.87503100 \\ \mathrm{C} & -2.00023200 & -1.53036500 & -2.22934900 \\ \mathrm{C} & -2.97981900 & -0.75177500 & -1.57251000 \\ \mathrm{C} & 1.37188500 & 3.54733200 & 0.56500900 \\ \mathrm{C} & 2.52369300 & 2.93718500 & 1.16705100 \\ \mathrm{C} & 2.18838700 & 2.48590100 & 2.47230500 \\ \mathrm{C} & 0.82383200 & 2.80160700 & 2.69069100 \\ \mathrm{C} & 0.32593000 & 3.45020200 & 1.53161200 \\ \mathrm{H} & 0.70955700 & 0.60373400 & -0.87740900 \\ \mathrm{H} & 2.41001100 & 0.58479200 & 0.03563200\end{array}$




$\begin{array}{lrrr}\mathrm{H} & 1.46531700 & -0.39450600 & 1.56018200 \\ \mathrm{H} & -0.87027600 & 1.07981800 & 0.72895300 \\ \mathrm{H} & 2.84910700 & 1.97833900 & 3.16335000 \\ \mathrm{H} & 3.49854200 & 2.84233600 & 0.70506000 \\ \mathrm{H} & 0.24807900 & 2.55757700 & 3.57692000 \\ \mathrm{H} & -0.69355800 & 3.78729200 & 1.38993700 \\ \mathrm{H} & -0.13451700 & 4.38258800 & -1.58122000 \\ \mathrm{H} & 1.75466900 & 5.77855500 & -1.04642700 \\ \mathrm{H} & 2.12810200 & 3.64928100 & -2.09174200 \\ \mathrm{H} & 2.55538400 & -1.00319000 & -3.18294200 \\ \mathrm{H} & 4.40040900 & -0.59342300 & -1.28430300 \\ \mathrm{H} & 1.08680200 & -3.14561200 & -2.47512300 \\ \mathrm{H} & 2.00853400 & -4.04346700 & -0.12396300 \\ \mathrm{H} & 5.69625900 & -3.53935500 & 1.00195200 \\ \mathrm{H} & 5.15334000 & -1.29087800 & 1.66696700 \\ \mathrm{H} & 3.76182000 & -3.15708100 & 2.37516500 \\ \mathrm{H} & -4.29610400 & 1.85385200 & 0.12560600 \\ \mathrm{H} & -2.84675400 & 3.31228100 & -1.16599900 \\ \mathrm{H} & -4.82680600 & 2.37182300 & -2.15641400 \\ \mathrm{H} & -1.03275100 & 1.58644600 & -2.94511500 \\ \mathrm{H} & -0.23774500 & -0.92813200 & -3.47642300 \\ \mathrm{H} & -1.93610900 & -2.61138800 & -2.21508500 \\ \mathrm{H} & -3.78009700 & -1.14347900 & -0.95448500 \\ \mathrm{C} & -4.95972800 & -2.26469100 & 1.63061500 \\ \mathrm{C} & -4.66631400 & -0.96924700 & 2.06465100 \\ \mathrm{C} & -3.33815900 & -0.58612900 & 2.26958900 \\ \mathrm{C} & -2.30300900 & -1.50019700 & 2.04006700 \\ \mathrm{C} & -2.59952200 & -2.79750600 & 1.60321400 \\ \mathrm{C} & -3.92744000 & -3.17931000 & 1.40010900 \\ \mathrm{H} & -5.99332200 & -2.56345600 & 1.47603800 \\ \mathrm{H} & -5.47001200 & -0.26041900 & 2.24425700 \\ \mathrm{H} & -3.10633000 & 0.42065000 & 2.60547400 \\ \mathrm{H} & -1.26918200 & -1.21485600 & 2.22198300 \\ \mathrm{H} & -1.79253100 & -3.50217200 & 1.42379000 \\ \mathrm{H} & -4.18761600 & 1.06706000 \\ \mathrm{H} & -1.04721200 & -0.23261000 \\ \mathrm{H} & -1.81977900 & -0.06062200\end{array}$

$\begin{array}{cccc}\text { TS5 }^{\mathbf{C S}} & & & \\ \mathrm{Ti} & -1.11587000 & -1.01934100 & -0.42395500 \\ \mathrm{Ti} & -0.05358900 & 1.27102700 & 0.57590600 \\ \mathrm{Si} & 4.97190600 & 0.50662500 & 0.65659900 \\ \mathrm{Si} & -4.70114900 & -0.77176300 & 0.72044300 \\ \mathrm{Si} & -1.68447800 & 3.85342400 & -1.62378700 \\ \mathrm{C} & 3.93406500 & -0.19967700 & -0.72235200 \\ \mathrm{C} & 3.32000700 & 0.52540800 & -1.79810000\end{array}$




\begin{tabular}{|c|c|c|c|}
\hline $\mathrm{C}$ & 2.67102300 & -0.38583900 & -2.66707200 \\
\hline $\mathrm{C}$ & 2.87981000 & -1.69454200 & -2.14842200 \\
\hline $\mathrm{C}$ & 3.65364400 & -1.57680600 & -0.96842800 \\
\hline $\mathrm{C}$ & -3.49864400 & -1.24093200 & -0.62139000 \\
\hline $\mathrm{C}$ & -2.99353400 & -0.37366100 & -1.64878500 \\
\hline $\mathrm{C}$ & -2.16825900 & -1.13013500 & -2.51870700 \\
\hline $\mathrm{C}$ & -2.15715100 & -2.47449700 & -2.04944200 \\
\hline $\mathrm{C}$ & -2.97432200 & -2.54028800 & -0.90079000 \\
\hline $\mathrm{C}$ & -0.95474400 & 3.42382500 & 0.03797800 \\
\hline $\mathrm{C}$ & 0.41426300 & 3.56967900 & 0.44260600 \\
\hline $\mathrm{C}$ & 0.53871300 & 3.20781600 & 1.81117500 \\
\hline $\mathrm{C}$ & -0.75044200 & 2.82663800 & 2.26711100 \\
\hline $\mathrm{C}$ & -1.66025400 & 2.96068300 & 1.18885400 \\
\hline $\mathrm{H}$ & -0.17632600 & 0.41883200 & -1.06961500 \\
\hline $\mathrm{H}$ & 1.36474300 & 1.35723500 & -0.55221100 \\
\hline $\mathrm{H}$ & 1.43793400 & 0.34382200 & 1.09117000 \\
\hline $\mathrm{H}$ & -1.51840100 & 0.27418300 & 0.78744600 \\
\hline $\mathrm{H}$ & 1.45157600 & 3.21310600 & 2.39320400 \\
\hline $\mathrm{H}$ & 1.22390500 & 3.91268200 & -0.18959000 \\
\hline $\mathrm{H}$ & -0.99428200 & 2.46203300 & 3.25891500 \\
\hline $\mathrm{H}$ & -2.71405300 & 2.71335500 & 1.22332600 \\
\hline $\mathrm{H}$ & -2.97611300 & 3.14098600 & -1.81057700 \\
\hline $\mathrm{H}$ & -1.95615000 & 5.31589000 & -1.72905700 \\
\hline $\mathrm{H}$ & -0.75034000 & 3.49318500 & -2.72313100 \\
\hline $\mathrm{H}$ & 2.10953000 & -0.13294600 & -3.55733700 \\
\hline $\mathrm{H}$ & 3.34854700 & 1.60013800 & -1.92434000 \\
\hline $\mathrm{H}$ & 2.50221100 & -2.61493300 & -2.57533800 \\
\hline $\mathrm{H}$ & 3.97337100 & -2.40117800 & -0.34068800 \\
\hline $\mathrm{H}$ & 6.40785700 & 0.59314900 & 0.26396600 \\
\hline $\mathrm{H}$ & 4.51644900 & 1.87724500 & 1.00595500 \\
\hline $\mathrm{H}$ & 4.89079100 & -0.37108300 & 1.85604800 \\
\hline $\mathrm{H}$ & -4.43823700 & -1.55524100 & 1.96059400 \\
\hline $\mathrm{H}$ & -4.60173100 & 0.67944000 & 1.02682000 \\
\hline $\mathrm{H}$ & -6.10936500 & -1.05373200 & 0.31921600 \\
\hline $\mathrm{H}$ & -3.20637900 & 0.68303900 & -1.73969300 \\
\hline $\mathrm{H}$ & -1.62814300 & -0.75169400 & -3.37674100 \\
\hline $\mathrm{H}$ & -1.59892100 & -3.29443900 & -2.48509000 \\
\hline $\mathrm{H}$ & -3.14985300 & -3.43021700 & -0.30590400 \\
\hline $\mathrm{C}$ & -0.24719100 & -2.91189300 & 0.95811400 \\
\hline $\mathrm{C}$ & -1.11729400 & -2.25302200 & 1.85076900 \\
\hline $\mathrm{C}$ & -0.55929800 & -1.52075400 & 2.94363600 \\
\hline $\mathrm{C}$ & 0.80066300 & -1.39359200 & 3.09678400 \\
\hline $\mathrm{C}$ & 1.68260700 & -1.98196000 & 2.13783200 \\
\hline $\mathrm{C}$ & 1.15858200 & -2.74858800 & 1.10785200 \\
\hline $\mathrm{H}$ & -0.61535200 & -3.66123200 & 0.26571600 \\
\hline $\mathrm{H}$ & -2.17707500 & -2.47936600 & 1.85804500 \\
\hline
\end{tabular}




$\begin{array}{cccc}\mathrm{H} & -1.23548600 & -1.07313100 & 3.66798700 \\ \mathrm{H} & 1.21404000 & -0.83776400 & 3.93317900 \\ \mathrm{H} & 2.75696600 & -1.89911300 & 2.26686800 \\ \mathrm{H} & 1.81829000 & -3.29306600 & 0.43817700 \\ \mathrm{Ti} & 1.55133000 & -0.45133800 & -0.57433600 \\ \mathrm{H} & 0.38746000 & -1.60003900 & -1.28575600\end{array}$

\begin{tabular}{|c|c|c|c|}
\hline$A 6^{C}$ & & & \\
\hline $\mathrm{Ti}$ & 1.46434400 & -0.77405100 & 0.25714900 \\
\hline $\mathrm{Ti}$ & -1.24068900 & -1.05005000 & 0.20016500 \\
\hline $\mathrm{Ti}$ & -0.12414500 & 1.29818800 & -0.48202800 \\
\hline $\mathrm{Si}$ & -4.84396300 & -0.59120800 & -0.79073400 \\
\hline $\mathrm{Si}$ & 5.08186400 & -0.09651700 & -0.70817100 \\
\hline $\mathrm{Si}$ & -0.85160600 & 3.34018600 & 2.53755200 \\
\hline $\mathrm{C}$ & -3.61401600 & -1.41878600 & 0.33929800 \\
\hline $\mathrm{C}$ & -3.15927400 & -0.93445100 & 1.61174200 \\
\hline $\mathrm{C}$ & -2.28261600 & -1.88221800 & 2.17947300 \\
\hline $\mathrm{C}$ & -2.18785500 & -2.98080700 & 1.27517500 \\
\hline $\mathrm{C}$ & -3.01407700 & -2.70447400 & 0.16413200 \\
\hline $\mathrm{C}$ & 3.88414700 & -0.81370100 & 0.52290800 \\
\hline $\mathrm{C}$ & 3.32285200 & -0.12888400 & 1.64845000 \\
\hline $\mathrm{C}$ & 2.52531700 & -1.02709700 & 2.38911400 \\
\hline $\mathrm{C}$ & 2.59223000 & -2.29636900 & 1.74232600 \\
\hline $\mathrm{C}$ & 3.42488100 & -2.16640600 & 0.60925600 \\
\hline $\mathrm{C}$ & -0.53087500 & 3.32346300 & 0.69952700 \\
\hline $\mathrm{C}$ & -1.51401200 & 3.22902300 & -0.34038900 \\
\hline $\mathrm{C}$ & -0.87104300 & 3.32245900 & -1.59613400 \\
\hline $\mathrm{C}$ & 0.52104100 & 3.46298600 & -1.35971100 \\
\hline $\mathrm{C}$ & 0.72675000 & 3.47527100 & 0.04182400 \\
\hline $\mathrm{H}$ & 0.17811900 & -1.79769800 & 1.01614200 \\
\hline $\mathrm{H}$ & 0.06315600 & 0.06433100 & 0.97994300 \\
\hline $\mathrm{H}$ & -1.71432700 & 0.42254300 & -0.80072300 \\
\hline $\mathrm{H}$ & -1.38277500 & 0.71435800 & 0.77961500 \\
\hline $\mathrm{H}$ & 1.47953800 & 1.04663400 & 0.30532400 \\
\hline $\mathrm{H}$ & -1.35452100 & 3.26857600 & -2.56369000 \\
\hline $\mathrm{H}$ & -2.57760200 & 3.09067300 & -0.18692700 \\
\hline $\mathrm{H}$ & 1.29477000 & 3.53051500 & -2.11535100 \\
\hline $\mathrm{H}$ & 1.68728700 & 3.56655200 & 0.53312200 \\
\hline $\mathrm{H}$ & 0.25272900 & 2.67066700 & 3.27336000 \\
\hline $\mathrm{H}$ & -0.95401200 & 4.73654000 & 3.05025700 \\
\hline $\mathrm{H}$ & -2.13780200 & 2.65523800 & 2.83181600 \\
\hline $\mathrm{H}$ & -1.75686000 & -1.78405700 & 3.12058500 \\
\hline $\mathrm{H}$ & -3.42830400 & 0.01650300 & 2.05429900 \\
\hline $\mathrm{H}$ & -1.58115200 & -3.86702600 & 1.41512000 \\
\hline $\mathrm{H}$ & -3.15915700 & -3.35705400 & -0.68769600 \\
\hline $\mathrm{H}$ & -6.22293900 & -1.11624100 & -0.58189400 \\
\hline
\end{tabular}




$\begin{array}{lrrc}\mathrm{H} & -4.86769500 & 0.87302300 & -0.53314200 \\ \mathrm{H} & -4.49033200 & -0.83378000 & -2.21722400 \\ \mathrm{H} & 4.80607100 & -0.60495100 & -2.08151500 \\ \mathrm{H} & 4.97234900 & 1.38757900 & -0.71051200 \\ \mathrm{H} & 6.49165600 & -0.45260400 & -0.38288700 \\ \mathrm{H} & 3.46727900 & 0.91917700 & 1.87950500 \\ \mathrm{H} & 1.95397800 & -0.79170300 & 3.27796700 \\ \mathrm{H} & 2.08625600 & -3.19908800 & 2.06074700 \\ \mathrm{H} & 3.66567200 & -2.96168800 & -0.08645700 \\ \mathrm{C} & 0.10446400 & 0.50101700 & -2.53392700 \\ \mathrm{C} & -1.01444200 & -0.37297900 & -2.44242400 \\ \mathrm{C} & -0.93742400 & -1.69162300 & -1.89990000 \\ \mathrm{C} & 0.38605800 & -2.15613500 & -1.47660800 \\ \mathrm{C} & 1.56192600 & -1.40777700 & -1.79321000 \\ \mathrm{C} & 1.39405800 & -0.00037200 & -2.04126900 \\ \mathrm{H} & 0.07011600 & 1.30015600 & -3.26745700 \\ \mathrm{H} & -1.93233900 & -0.08600100 & -2.95034500 \\ \mathrm{H} & -1.68802500 & -2.40831900 & -2.21614100 \\ \mathrm{H} & 0.49615200 & -3.22147700 & -1.28920700 \\ \mathrm{H} & 2.50055000 & -1.90384000 & -2.01248700 \\ \mathrm{H} & 2.27623500 & 0.56522300 & -2.32942200\end{array}$

\section{TS6 $^{\mathrm{CS}}$}

Ti

$\mathrm{Ti}$

$\mathrm{Ti}$

$\mathrm{Si}$

$\mathrm{Si}$

$\mathrm{Si}$

C

C

C

C

C

C

C

C

C

C

C

C

C

C

C

$\mathrm{H}$

$\mathrm{H}$

$\begin{array}{rrr}1.45447000 & -0.78831100 & 0.25654400 \\ -1.25273300 & -1.01318700 & 0.21940800 \\ -0.10640200 & 1.29528900 & -0.48178700 \\ -4.83786300 & -0.67615700 & -0.87234100 \\ 5.07567900 & -0.10979400 & -0.67843600 \\ -0.88883400 & 3.33836100 & 2.52354700 \\ -3.62166700 & -1.41800800 & 0.32863000 \\ -3.20069300 & -0.85236200 & 1.57851100 \\ -2.32114100 & -1.75114700 & 2.22059100 \\ -2.19043900 & -2.89725100 & 1.38506600 \\ -2.99333300 & -2.69849300 & 0.23941400 \\ 3.86785000 & -0.84123100 & 0.53423500 \\ 3.29773400 & -0.16995100 & 1.66427400 \\ 2.49619800 & -1.07825200 & 2.38985000 \\ 2.56644000 & -2.33819900 & 1.72750500 \\ 3.40649400 & -2.19406900 & 0.60111000 \\ -0.51737400 & 3.32483100 & 0.69491600 \\ -1.47327800 & 3.24896400 & -0.37150100 \\ -0.79476900 & 3.33760200 & -1.60833900 \\ 0.59246300 & 3.45673700 & -1.33402500 \\ 0.75993700 & 3.46087300 & 0.07245700 \\ 0.17654900 & -1.89786600 & 0.87607600 \\ 0.06894100 & -0.00427000 & 1.05656200\end{array}$




$\begin{array}{lrrc}\mathrm{H} & -1.71574300 & 0.45162700 & -0.81596900 \\ \mathrm{H} & -1.36352600 & 0.75203200 & 0.77500600 \\ \mathrm{H} & 1.47042800 & 1.02324700 & 0.35732400 \\ \mathrm{H} & -1.25305000 & 3.29559000 & -2.58864700 \\ \mathrm{H} & -2.54254900 & 3.12624900 & -0.24746900 \\ \mathrm{H} & 1.38715500 & 3.51599600 & -2.06827500 \\ \mathrm{H} & 1.70800400 & 3.53683900 & 0.58986100 \\ \mathrm{H} & 0.16654600 & 2.62106400 & 3.28564700 \\ \mathrm{H} & -0.95176500 & 4.73376200 & 3.04531700 \\ \mathrm{H} & -2.20866100 & 2.70180300 & 2.77362400 \\ \mathrm{H} & -1.81821500 & -1.58908700 & 3.16542800 \\ \mathrm{H} & -3.49434400 & 0.11815100 & 1.95872400 \\ \mathrm{H} & -1.57584500 & -3.76499600 & 1.59000900 \\ \mathrm{H} & -3.10368700 & -3.40028900 & -0.57815400 \\ \mathrm{H} & -6.20834400 & -1.23058200 & -0.68423700 \\ \mathrm{H} & -4.90839600 & 0.79649400 & -0.67880600 \\ \mathrm{H} & -4.43444300 & -0.96742800 & -2.27674000 \\ \mathrm{H} & 4.81491600 & -0.60907100 & -2.05821000 \\ \mathrm{H} & 4.96076400 & 1.37381700 & -0.67099500 \\ \mathrm{H} & 6.48357000 & -0.46323800 & -0.34238700 \\ \mathrm{H} & 3.44332200 & 0.87419100 & 1.91132000 \\ \mathrm{H} & 1.92007200 & -0.85418500 & 3.27836800 \\ \mathrm{H} & 2.05815400 & -3.24472300 & 2.03079800 \\ \mathrm{H} & 3.65160400 & -2.98134900 & -0.10226200 \\ \mathrm{C} & 0.12876900 & 0.51400500 & -2.53637200 \\ \mathrm{C} & -1.00209300 & -0.33372800 & -2.43033900 \\ \mathrm{C} & -0.94179700 & -1.66315500 & -1.89123600 \\ \mathrm{C} & 0.39078700 & -2.17231800 & -1.57613700 \\ \mathrm{C} & 1.55808900 & -1.41646100 & -1.82940000 \\ \mathrm{C} & 1.41042800 & 0.00747900 & -2.01693100 \\ \mathrm{H} & 0.10554000 & 1.31564900 & -3.26713400 \\ \mathrm{H} & -1.91768900 & -0.03492000 & -2.93515800 \\ & -1.71168400 & -2.36145300 & -2.20512400 \\ \mathrm{H} & 0.49108700 & -3.24146000 & -1.40746400 \\ \mathrm{H} & 2.50686500 & -1.90793500 & -2.01029800 \\ \mathrm{H} & 2.29964200 & 0.56399200 & -2.30080800\end{array}$

$\begin{array}{cccc}\mathbf{A} 7^{\mathbf{C S}} & & & \\ \mathrm{Ti} & 1.44100700 & -0.82637800 & 0.24426000 \\ \mathrm{Ti} & -1.19765000 & -1.09142800 & 0.15476600 \\ \mathrm{Ti} & -0.14084800 & 1.25597300 & -0.49545200 \\ \mathrm{Si} & -4.79432700 & -0.38052200 & -0.67820400 \\ \mathrm{Si} & 5.01147900 & 0.31993000 & -0.41916300 \\ \mathrm{Si} & -1.11471900 & 3.07459700 & 2.60128100 \\ \mathrm{C} & -3.57732500 & -1.38698500 & 0.31411500 \\ \mathrm{C} & -3.07508300 & -1.07021700 & 1.61943900\end{array}$




\begin{tabular}{|c|c|c|c|}
\hline $\mathrm{C}$ & -2.23448200 & -2.11842600 & 2.05333200 \\
\hline $\mathrm{C}$ & -2.20110000 & -3.10445100 & 1.02711400 \\
\hline $\mathrm{C}$ & -3.03279400 & -2.66318300 & -0.02854600 \\
\hline $\mathrm{C}$ & 3.83714500 & -0.69256800 & 0.61270100 \\
\hline $\mathrm{C}$ & 3.17171300 & -0.26229200 & 1.80614900 \\
\hline $\mathrm{C}$ & 2.45380100 & -1.34864400 & 2.35332400 \\
\hline $\mathrm{C}$ & 2.66159900 & -2.47626800 & 1.50945700 \\
\hline $\mathrm{C}$ & 3.51215200 & -2.07648000 & 0.45341300 \\
\hline $\mathrm{C}$ & -0.65233400 & 3.19259900 & 0.79811300 \\
\hline $\mathrm{C}$ & -1.54464400 & 3.19149400 & -0.32224900 \\
\hline $\mathrm{C}$ & -0.79504600 & 3.37753600 & -1.50655100 \\
\hline $\mathrm{C}$ & 0.57360600 & 3.49391400 & -1.14479700 \\
\hline $\mathrm{C}$ & 0.65936400 & 3.38725900 & 0.26302600 \\
\hline $\mathrm{H}$ & 0.27326500 & -2.21868700 & 0.26566800 \\
\hline $\mathrm{H}$ & 0.08848700 & -0.89238200 & 1.43325600 \\
\hline $\mathrm{H}$ & -1.77286300 & 0.46568500 & -0.68142900 \\
\hline $\mathrm{H}$ & -1.01765000 & 0.55656600 & 0.92079500 \\
\hline $\mathrm{H}$ & 1.20005600 & 0.90569900 & 0.64913200 \\
\hline $\mathrm{H}$ & -1.19654300 & 3.40681200 & -2.51220100 \\
\hline $\mathrm{H}$ & -2.61757600 & 3.05250500 & -0.27084200 \\
\hline $\mathrm{H}$ & 1.40723600 & 3.62471400 & -1.82480000 \\
\hline $\mathrm{H}$ & 1.57414500 & 3.43111000 & 0.84067600 \\
\hline $\mathrm{H}$ & -0.07489400 & 2.34234700 & 3.36993300 \\
\hline $\mathrm{H}$ & -1.24131100 & 4.43339100 & 3.20462500 \\
\hline $\mathrm{H}$ & -2.42794700 & 2.39509400 & 2.75330200 \\
\hline $\mathrm{H}$ & -1.69050500 & -2.15321000 & 2.98845500 \\
\hline $\mathrm{H}$ & -3.28486300 & -0.16158000 & 2.16990100 \\
\hline $\mathrm{H}$ & -1.63739400 & -4.02888000 & 1.05235400 \\
\hline $\mathrm{H}$ & -3.22282100 & -3.20369900 & -0.94789500 \\
\hline $\mathrm{H}$ & -6.18606900 & -0.88528100 & -0.50163800 \\
\hline $\mathrm{H}$ & -4.76508100 & 1.03902200 & -0.23961800 \\
\hline $\mathrm{H}$ & -4.48107000 & -0.45649900 & -2.13210000 \\
\hline $\mathrm{H}$ & 4.85984200 & -0.00714600 & -1.86508000 \\
\hline $\mathrm{H}$ & 4.74486100 & 1.76894200 & -0.21641200 \\
\hline $\mathrm{H}$ & 6.43136700 & 0.05531600 & -0.05433300 \\
\hline $\mathrm{H}$ & 3.20278000 & 0.73906800 & 2.21641000 \\
\hline $\mathrm{H}$ & 1.84306000 & -1.32358600 & 3.24601200 \\
\hline $\mathrm{H}$ & 2.23937700 & -3.46313200 & 1.65099000 \\
\hline $\mathrm{H}$ & 3.85790800 & -2.71927200 & -0.34735900 \\
\hline $\mathrm{C}$ & 0.20859600 & 0.62271700 & -2.60065800 \\
\hline $\mathrm{C}$ & -0.88461600 & -0.23945700 & -2.57768300 \\
\hline $\mathrm{C}$ & -0.79990800 & -1.59358000 & -2.07009500 \\
\hline $\mathrm{C}$ & 0.55299400 & -2.09520000 & -1.91711700 \\
\hline $\mathrm{C}$ & 1.66788800 & -1.26205800 & -1.93077900 \\
\hline $\mathrm{C}$ & 1.44769200 & 0.20055000 & -1.91033500 \\
\hline $\mathrm{H}$ & 0.19423900 & 1.48803100 & -3.25351400 \\
\hline
\end{tabular}




$\begin{array}{cccc}\mathrm{H} & -1.80573700 & 0.06464800 & -3.06899500 \\ \mathrm{H} & -1.55602400 & -2.29744700 & -2.40538500 \\ \mathrm{H} & 0.70135200 & -3.17251700 & -1.91837900 \\ \mathrm{H} & 2.64953100 & -1.67944300 & -2.12468600 \\ \mathrm{H} & 2.32627200 & 0.80052100 & -2.13847300\end{array}$

TS7 ${ }^{\mathrm{CS}}$

$\mathrm{Ti}$

Ti

$\mathrm{Ti}$

$\mathrm{Si}$

$\mathrm{Si}$

$\mathrm{Si}$

C

C

C

C

C

C

C

C

C

C

C

C

C

C

C

$\mathrm{H}$

$\mathrm{H}$

$\mathrm{H}$

H

$\mathrm{H}$

$\mathrm{H}$

$\mathrm{H}$

$\mathrm{H}$

$\mathrm{H}$

$\mathrm{H}$

$\mathrm{H}$

$\mathrm{H}$

$\mathrm{H}$

$\mathrm{H}$

$\mathrm{H}$

$\mathrm{H}$

$\mathrm{H}$

$\mathrm{H}$

$\begin{array}{ccc}-1.13917100 & -1.07775000 & -0.13208700 \\ 1.56332800 & -0.86086500 & -0.19686000 \\ 0.11242400 & 1.30639400 & 0.32760900 \\ 4.97768900 & 0.67786200 & 0.04979200 \\ -4.80434700 & -0.73043700 & 0.84091600 \\ -2.41348100 & 3.11113100 & -1.83518900 \\ 3.91883300 & -0.70165700 & -0.62467200 \\ 3.23503700 & -0.71585400 & -1.88546300 \\ 2.60854500 & -1.96993300 & -2.05076300 \\ 2.89036500 & -2.75600500 & -0.89766900 \\ 3.70464400 & -1.98584500 & -0.03530200 \\ -3.52655300 & -1.50336300 & -0.27449000 \\ -3.08983900 & -1.01247800 & -1.54586000 \\ -2.18443100 & -1.93554200 & -2.11276000 \\ -2.04790400 & -3.02434400 & -1.20454500 \\ -2.87289000 & -2.76274600 & -0.08661100 \\ -0.93836600 & 3.21698700 & -0.69823100 \\ 0.43439000 & 3.19998000 & -1.08835700 \\ 1.23927200 & 3.41753400 & 0.05953000 \\ 0.37554300 & 3.57435100 & 1.17322600 \\ -0.95751900 & 3.43974100 & 0.71476500 \\ 0.29199900 & -2.24038300 & 0.23378800 \\ 0.21244300 & -1.27064100 & -1.32392100 \\ 1.87693400 & 0.85705600 & 0.36079700 \\ 0.88186200 & 0.55256500 & -1.12426300 \\ -1.22211900 & 0.63974600 & -0.68323500 \\ 2.32110300 & 3.44851900 & 0.08046500 \\ 0.80205000 & 3.03813100 & -2.09372700 \\ 0.68635300 & 3.75266700 & 2.19508300 \\ -1.84822200 & 3.49135700 & 1.33084000 \\ -3.58744900 & 2.54748900 & -1.11928500 \\ -2.79441900 & 4.46855100 & -2.32208500 \\ -2.10249500 & 2.27804600 & -3.02591200 \\ 2.00072300 & -2.27142000 & -2.89397000 \\ 3.18625600 & 0.10977100 & -2.58458800 \\ 2.54678200 & -3.76812900 & -0.71912400 \\ 4.09831700 & -2.31420700 & 0.91878800 \\ 6.41898100 & 0.45262200 & -0.25841600 \\ 4.58109700 & 1.97500900 & -0.55798900\end{array}$

S46 


$\begin{array}{lrrr}\mathrm{H} & 4.85077800 & 0.76316000 & 1.53048200 \\ \mathrm{H} & -4.44158000 & -0.93938300 & 2.27096400 \\ \mathrm{H} & -4.90918800 & 0.72683200 & 0.57071700 \\ \mathrm{H} & -6.14785900 & -1.34018300 & 0.63396600 \\ \mathrm{H} & -3.38814700 & -0.07254700 & -1.99158200 \\ \mathrm{H} & -1.67057300 & -1.82452700 & -3.05866500 \\ \mathrm{H} & -1.41812800 & -3.89442900 & -1.34488400 \\ \mathrm{H} & -2.99086300 & -3.41313000 & 0.77163200 \\ \mathrm{C} & 0.01717700 & 0.77921200 & 2.49318300 \\ \mathrm{C} & 1.25695100 & 0.14151600 & 2.44997900 \\ \mathrm{C} & 1.43791000 & -1.21950900 & 2.00421700 \\ \mathrm{C} & 0.20059700 & -1.99023500 & 1.79276400 \\ \mathrm{C} & -1.09280100 & -1.39239400 & 1.96420000 \\ \mathrm{C} & -1.15347000 & 0.08269700 & 1.94435000 \\ \mathrm{H} & -0.09593500 & 1.68346500 & 3.08006900 \\ \mathrm{H} & 2.12497300 & 0.65516700 & 2.85740800 \\ \mathrm{H} & 2.30045800 & -1.75808800 & 2.38627800 \\ \mathrm{H} & 0.26931500 & -3.06191600 & 1.97391100 \\ \mathrm{H} & -1.89403100 & -1.98621800 & 2.39025300 \\ \mathrm{H} & -2.11518100 & 0.51144600 & 2.21941900\end{array}$

$\begin{array}{cccc}\mathbf{A 8}^{\mathbf{C S}} & & & \\ \mathrm{Ti} & -1.16096200 & -0.99572900 & -0.28904800 \\ \mathrm{Ti} & 1.69671500 & -0.89987800 & 0.10596500 \\ \mathrm{Ti} & 0.11694400 & 1.28160000 & 0.42239300 \\ \mathrm{Si} & 4.63621200 & 1.08211200 & -1.18858300 \\ \mathrm{Si} & -4.89184200 & -1.03575300 & 0.36787000 \\ \mathrm{Si} & -2.57077800 & 3.38624800 & -1.24245800 \\ \mathrm{C} & 3.88848900 & -0.59302400 & -0.84237800 \\ \mathrm{C} & 3.11730300 & -1.38027000 & -1.74910100 \\ \mathrm{C} & 2.81547700 & -2.62189600 & -1.13420900 \\ \mathrm{C} & 3.39261500 & -2.62368600 & 0.16352900 \\ \mathrm{C} & 4.04257900 & -1.38043100 & 0.34197100 \\ \mathrm{C} & -3.46481700 & -1.54306800 & -0.72718900 \\ \mathrm{C} & -2.96715000 & -0.82748500 & -1.86335100 \\ \mathrm{C} & -1.94910200 & -1.58972100 & -2.47562800 \\ \mathrm{C} & -1.80571400 & -2.79710000 & -1.73560200 \\ \mathrm{C} & -2.73625300 & -2.77256600 & -0.66959900 \\ \mathrm{C} & -0.94676000 & 3.32175200 & -0.32603500 \\ \mathrm{C} & 0.35425300 & 3.25322400 & -0.91241600 \\ \mathrm{C} & 1.32117300 & 3.35204000 & 0.11932500 \\ \mathrm{C} & 0.63579600 & 3.46741000 & 1.35543700 \\ \mathrm{C} & -0.75286900 & 3.44525300 & 1.08451500 \\ \mathrm{H} & 0.11598700 & -3.23563600 & 1.86234500 \\ \mathrm{H} & 0.36010100 & -1.99951200 & -0.49950200 \\ \mathrm{H} & 1.83901900 & 0.88828600 & -0.03558500\end{array}$




\begin{tabular}{|c|c|c|c|}
\hline $\mathrm{H}$ & 0.35374400 & 0.00517600 & -0.90751500 \\
\hline $\mathrm{H}$ & -1.37875600 & 0.78614500 & -0.49435000 \\
\hline $\mathrm{H}$ & 2.39483600 & 3.32890600 & -0.01219200 \\
\hline $\mathrm{H}$ & 0.56709600 & 3.14069400 & -1.96865600 \\
\hline $\mathrm{H}$ & 1.09665900 & 3.53808800 & 2.33340200 \\
\hline $\mathrm{H}$ & -1.54212000 & 3.50222200 & 1.82558500 \\
\hline $\mathrm{H}$ & -3.66189200 & 2.78581000 & -0.43148800 \\
\hline $\mathrm{H}$ & -2.95093000 & 4.79795300 & -1.53513600 \\
\hline $\mathrm{H}$ & -2.46460000 & 2.67238800 & -2.54254400 \\
\hline $\mathrm{H}$ & 2.23776300 & -3.42477000 & -1.57402900 \\
\hline $\mathrm{H}$ & 2.79602500 & -1.06899200 & -2.73623900 \\
\hline $\mathrm{H}$ & 3.32433500 & -3.42104400 & 0.89216900 \\
\hline $\mathrm{H}$ & 4.55564200 & -1.06290100 & 1.24334800 \\
\hline $\mathrm{H}$ & 6.01433400 & 0.93807100 & -1.73771300 \\
\hline $\mathrm{H}$ & 3.81905800 & 1.81431400 & -2.19039500 \\
\hline $\mathrm{H}$ & 4.73308700 & 1.88058500 & 0.06226000 \\
\hline $\mathrm{H}$ & -4.70369100 & -1.57516700 & 1.74144800 \\
\hline $\mathrm{H}$ & -4.99288500 & 0.44560200 & 0.42822200 \\
\hline $\mathrm{H}$ & -6.17882500 & -1.56893900 & -0.15813900 \\
\hline $\mathrm{H}$ & -3.29765400 & 0.15162700 & -2.18515500 \\
\hline $\mathrm{H}$ & -1.36127300 & -1.29193100 & -3.33550600 \\
\hline $\mathrm{H}$ & -1.10087900 & -3.59159200 & -1.94541400 \\
\hline $\mathrm{H}$ & -2.86335200 & -3.54938000 & 0.07373100 \\
\hline $\mathrm{C}$ & -0.15056200 & 0.63530800 & 2.47195200 \\
\hline $\mathrm{C}$ & 1.13592500 & 0.01834300 & 2.32524500 \\
\hline $\mathrm{C}$ & 1.29374500 & -1.42168100 & 2.11653000 \\
\hline $\mathrm{C}$ & 0.00672400 & -2.23986500 & 2.30763400 \\
\hline $\mathrm{C}$ & -1.24590500 & -1.54087900 & 1.75681000 \\
\hline $\mathrm{C}$ & -1.28452800 & -0.09016200 & 1.96964900 \\
\hline $\mathrm{H}$ & -0.28451400 & 1.48881400 & 3.12808200 \\
\hline $\mathrm{H}$ & 1.99073300 & 0.57780100 & 2.70571600 \\
\hline $\mathrm{H}$ & 2.15585300 & -1.85707900 & 2.62677800 \\
\hline $\mathrm{H}$ & -0.13821300 & -2.39858500 & 3.39308400 \\
\hline $\mathrm{H}$ & -2.16888200 & -2.05641900 & 2.02806700 \\
\hline $\mathrm{H}$ & -2.25795600 & 0.38214500 & 2.10235100 \\
\hline \multicolumn{4}{|l|}{$3^{\mathrm{CS}}$} \\
\hline $\mathrm{Ti}$ & 0.09061300 & 1.32248500 & -0.53911700 \\
\hline $\mathrm{Ti}$ & 1.25007300 & -0.96734400 & 0.17068900 \\
\hline $\mathrm{Ti}$ & -1.41111000 & -0.79724500 & 0.24627200 \\
\hline $\mathrm{Si}$ & 0.27010800 & 3.33526200 & 2.56809700 \\
\hline $\mathrm{Si}$ & 4.91686300 & -0.73953400 & -0.80378300 \\
\hline $\mathrm{Si}$ & -5.02441400 & -0.38342000 & -0.83778600 \\
\hline $\mathrm{C}$ & 1.16795100 & -0.15576200 & -2.03524400 \\
\hline $\mathrm{C}$ & -0.02421700 & 0.53569800 & -2.51719100 \\
\hline $\mathrm{C}$ & -1.29273300 & -0.07541500 & -2.23739700 \\
\hline
\end{tabular}




\begin{tabular}{|c|c|c|c|}
\hline $\mathrm{C}$ & -1.42498100 & -1.43254200 & -1.79055000 \\
\hline $\mathrm{C}$ & -0.21804100 & -2.09681200 & -1.32181700 \\
\hline $\mathrm{C}$ & 1.06917500 & -1.68423400 & -2.03977600 \\
\hline $\mathrm{C}$ & 0.28233000 & 3.33770900 & 0.70572500 \\
\hline $\mathrm{C}$ & 1.42758500 & 3.26441600 & -0.15215300 \\
\hline $\mathrm{C}$ & 1.00729000 & 3.36285800 & -1.49949400 \\
\hline $\mathrm{C}$ & -0.40426100 & 3.50287000 & -1.50357400 \\
\hline $\mathrm{C}$ & -0.84690800 & 3.49285300 & -0.15973400 \\
\hline $\mathrm{C}$ & 3.64580600 & -1.32128800 & 0.43013800 \\
\hline $\mathrm{C}$ & 3.17779900 & -0.58868900 & 1.57061600 \\
\hline $\mathrm{C}$ & 2.29118700 & -1.39904300 & 2.30851900 \\
\hline $\mathrm{C}$ & 2.21152500 & -2.66569700 & 1.65802000 \\
\hline $\mathrm{C}$ & 3.04616300 & -2.62120400 & 0.52520000 \\
\hline $\mathrm{C}$ & -3.34799000 & -0.25770000 & 1.57813500 \\
\hline $\mathrm{C}$ & -2.51362800 & -1.08665300 & 2.35595400 \\
\hline $\mathrm{C}$ & -2.47192400 & -2.36710000 & 1.72931400 \\
\hline $\mathrm{C}$ & -3.27766600 & -2.31453200 & 0.57058400 \\
\hline $\mathrm{C}$ & -3.82286600 & -0.99807900 & 0.44777700 \\
\hline $\mathrm{H}$ & -0.02411900 & 0.21835400 & 0.95177100 \\
\hline $\mathrm{H}$ & 1.62897400 & 0.75992900 & 0.26737800 \\
\hline $\mathrm{H}$ & -0.06961700 & -1.64568900 & 1.14191400 \\
\hline $\mathrm{H}$ & -1.56974600 & 1.00634100 & 0.12402200 \\
\hline $\mathrm{H}$ & 2.11234700 & 0.23160300 & -2.41926600 \\
\hline $\mathrm{H}$ & 0.04373600 & 1.29283800 & -3.29326600 \\
\hline $\mathrm{H}$ & -2.18651500 & 0.42552300 & -2.60329000 \\
\hline $\mathrm{H}$ & -2.31328800 & -1.99523000 & -2.06019500 \\
\hline $\mathrm{H}$ & -0.31284200 & -3.16846200 & -1.14149800 \\
\hline $\mathrm{H}$ & 1.96087100 & -2.15177900 & -1.57867300 \\
\hline $\mathrm{H}$ & -6.41983500 & -0.79994700 & -0.52210800 \\
\hline $\mathrm{H}$ & -4.98964200 & 1.10259500 & -0.89129400 \\
\hline $\mathrm{H}$ & -4.69338500 & -0.93067100 & -2.18255600 \\
\hline $\mathrm{H}$ & -3.56754000 & 0.78260400 & 1.78357500 \\
\hline $\mathrm{H}$ & -1.98513200 & -0.79763200 & 3.25570400 \\
\hline $\mathrm{H}$ & -1.91348200 & -3.22681500 & 2.07842600 \\
\hline $\mathrm{H}$ & -3.43747500 & -3.13318700 & -0.12109800 \\
\hline $\mathrm{H}$ & -1.87817200 & 3.57014900 & 0.16187900 \\
\hline $\mathrm{H}$ & -1.03708800 & 3.57665600 & -2.37967000 \\
\hline $\mathrm{H}$ & 1.64767400 & 3.31171900 & -2.37193000 \\
\hline $\mathrm{H}$ & 2.45253500 & 3.14495400 & 0.17718000 \\
\hline $\mathrm{H}$ & -1.03211000 & 2.82892800 & 3.07700900 \\
\hline $\mathrm{H}$ & 1.37379900 & 2.48888400 & 3.09763400 \\
\hline $\mathrm{H}$ & 0.46440800 & 4.70794900 & 3.11883100 \\
\hline $\mathrm{H}$ & 6.27516200 & -1.25566300 & -0.47673800 \\
\hline $\mathrm{H}$ & 4.57620700 & -1.22025900 & -2.17182900 \\
\hline $\mathrm{H}$ & 4.96926300 & 0.74588600 & -0.80203400 \\
\hline $\mathrm{H}$ & 3.19884900 & -3.43977000 & -0.16971400 \\
\hline
\end{tabular}




$\begin{array}{crrr}\mathrm{H} & 1.60822100 & -3.50689600 & 1.97514600 \\ \mathrm{H} & 1.75202900 & -1.10762600 & 3.20098300 \\ \mathrm{H} & 3.44919400 & 0.42957900 & 1.81686300 \\ \mathrm{H} & 1.10067300 & -2.08080100 & -3.06543100\end{array}$

\section{TS8 $^{\mathrm{CS}}$}

$\mathrm{Ti}$

$\mathrm{Ti}$

$\mathrm{Ti}$

$\mathrm{Si}$

$\mathrm{Si}$

$\mathrm{Si}$

C

C

C

C

C

C

C

C

C

C

C

C

C

C

C

C

C

C

C

C

C

$\mathrm{H}$

$\mathrm{H}$

$\mathrm{H}$

$\mathrm{H}$

$\mathrm{H}$

$\mathrm{H}$

$\mathrm{H}$

$\mathrm{H}$

$\mathrm{H}$

$\mathrm{H}$

$\mathrm{H}$

$\mathrm{H}$

$\mathrm{H}$

\begin{tabular}{|c|c|c|}
\hline-0.1668 & & \\
\hline 1.48390000 & -0.53492500 & 0.33274600 \\
\hline-1.20739900 & -1.01908600 & 0.28914800 \\
\hline-1.12570900 & 3.56072300 & 5300 \\
\hline 5.11220500 & -0.85027000 & -0.90 \\
\hline-4.75754100 & -1.37032900 & -1.02070300 \\
\hline 1.40549300 & 0.21990600 & -1.68252700 \\
\hline 0.27898300 & 0.28 & 21200 \\
\hline-0.90099500 & -0.483 & 3500 \\
\hline-0.91252500 & -1.81 & 9300 \\
\hline 0.29665100 & -2.35 & 7500 \\
\hline 1.6218 & -1.99 & 6000 \\
\hline-0.74796700 & 3.40 & 90000 \\
\hline 0.52605400 & 200 & 3100 \\
\hline & & 500 \\
\hline-1.00517000 & 3.18 & 5100 \\
\hline-1.68831300 & 3.17 & 6300 \\
\hline 3.9233 & -0.76 & 4000 \\
\hline 3.52228500 & 0.41 & 0000 \\
\hline 2.7109 & 0.0 & 6900 \\
\hline 2.60767 & -1.36 & 400 \\
\hline 3.32663000 & -1.85 & 6000 \\
\hline-3.29640900 & -0.76 & 000 \\
\hline-2.37545500 & -1.36 & 5200 \\
\hline-2.04946700 & -2.64 & 300 \\
\hline-2.77950400 & -2.83 & 400 \\
\hline-3.55481300 & -1.65 & 7700 \\
\hline-0.13280300 & 0.30 & 79200 \\
\hline 1.31544900 & 1.27 & 52400 \\
\hline 0.25990600 & -1.49 & 0000 \\
\hline-1.76145800 & 0.66 & 2800 \\
\hline 2.32777500 & 0.66 & 5500 \\
\hline 0.29042400 & 0.88 & 28400 \\
\hline-1.79820600 & -0.20583000 & 91800 \\
\hline-1.72406500 & -2.47793700 & -1.94883200 \\
\hline 0.19968800 & -3.27686400 & 55900 \\
\hline 2.42674700 & -2.58681200 & -1.08153600 \\
\hline-6.06890200 & -2.02448500 & -0.75334500 \\
\hline-4.99489700 & 0.08879000 & -1.18340500 \\
\hline-4.23375400 & -1.92918000 & -2.29742200 \\
\hline
\end{tabular}




$\begin{array}{lrrr}\mathrm{H} & -3.71798600 & 0.22700200 & 1.57265900 \\ \mathrm{H} & -1.96868400 & -0.91670300 & 3.24608100 \\ \mathrm{H} & -1.36238500 & -3.35813800 & 2.26867500 \\ \mathrm{H} & -2.73737400 & -3.71150400 & -0.00460100 \\ \mathrm{H} & -2.74856500 & 3.00290100 & -0.51955400 \\ \mathrm{H} & -1.45012200 & 3.01032000 & -2.86112000 \\ \mathrm{H} & 1.16056600 & 3.48423300 & -2.36856800 \\ \mathrm{H} & 1.46064700 & 3.76839100 & 0.28054500 \\ \mathrm{H} & -2.35020800 & 2.78691700 & 2.55907200 \\ \mathrm{H} & 0.01682900 & 3.06658100 & 3.03673400 \\ \mathrm{H} & -1.36810700 & 4.98084700 & 2.60621700 \\ \mathrm{H} & 6.52341500 & -0.90417300 & -0.43370600 \\ \mathrm{H} & 4.86046300 & -2.07297100 & -1.71598200 \\ \mathrm{H} & 4.97284600 & 0.35311000 & -1.77211100 \\ \mathrm{H} & 3.42565500 & -2.90630500 & 0.96937600 \\ \mathrm{H} & 2.04695200 & -1.95291600 & 3.05380600 \\ \mathrm{H} & 2.23883000 & 0.73386800 & 3.02341500 \\ \mathrm{H} & 3.78545300 & 1.42874400 & 0.94665000 \\ \mathrm{H} & 1.80827100 & -1.79570900 & -2.56185800\end{array}$

$\begin{array}{cccc}\text { B1 }^{\text {CS }} & & & \\ \mathrm{Ti} & -0.20153500 & 1.30903400 & -0.58937700 \\ \mathrm{Ti} & 1.52253600 & -0.47461900 & 0.37736400 \\ \mathrm{Ti} & -1.21109900 & -1.00611200 & 0.31375300 \\ \mathrm{Si} & -1.28264400 & 3.63707200 & 2.10665200 \\ \mathrm{Si} & 5.10665900 & -0.80816000 & -0.92406300 \\ \mathrm{Si} & -4.72409600 & -1.50630500 & -1.05668500 \\ \mathrm{C} & 1.50647400 & 0.32186700 & -1.58744400 \\ \mathrm{C} & 0.44414000 & 0.20491900 & -2.49352900 \\ \mathrm{C} & -0.73181800 & -0.58432300 & -2.23017500 \\ \mathrm{C} & -0.80060700 & -1.89969100 & -1.60962300 \\ \mathrm{C} & 0.34748000 & -2.58339000 & -1.06845100 \\ \mathrm{C} & 1.70104800 & -2.34719200 & -1.31887500 \\ \mathrm{C} & -0.87711900 & 3.41160000 & 0.30023700 \\ \mathrm{C} & 0.39877500 & 3.59840200 & -0.32440600 \\ \mathrm{C} & 0.26728300 & 3.38756000 & -1.71712800 \\ \mathrm{C} & -1.09289400 & 3.07209300 & -1.98273600 \\ \mathrm{C} & -1.79232500 & 3.09744600 & -0.75299800 \\ \mathrm{C} & 3.95884000 & -0.65636900 & 0.53595700 \\ \mathrm{C} & 3.54128300 & 0.55088400 & 1.16708000 \\ \mathrm{C} & 2.75352000 & 0.23831800 & 2.30897900 \\ \mathrm{C} & 2.67736100 & -1.17065600 & 2.40484300 \\ \mathrm{C} & 3.39197700 & -1.71805400 & 1.31288300 \\ \mathrm{C} & -3.33029600 & -0.75865400 & 1.42712900 \\ \mathrm{C} & -2.41604100 & -1.29568200 & 2.35728700 \\ \mathrm{C} & -2.04470500 & -2.59139000 & 1.89838600\end{array}$




$\begin{array}{lrrc}\mathrm{C} & -2.74302600 & -2.84484700 & 0.69208000 \\ \mathrm{C} & -3.54299400 & -1.70687300 & 0.37435500 \\ \mathrm{H} & -0.16026200 & 0.33210200 & 1.00952400 \\ \mathrm{H} & 1.26285100 & 1.34608900 & 0.41545100 \\ \mathrm{H} & 0.27088300 & -1.53655500 & 1.22317400 \\ \mathrm{H} & -1.80123000 & 0.62929100 & -0.13887300 \\ \mathrm{H} & 2.35500100 & 0.91900400 & -1.93583800 \\ \mathrm{H} & 0.43052100 & 0.78754300 & -3.41900100 \\ \mathrm{H} & -1.59708100 & -0.34257900 & -2.84761800 \\ \mathrm{H} & -1.66016500 & -2.51596900 & -1.86522100 \\ \mathrm{H} & 0.11940000 & -3.43745700 & -0.42673900 \\ \mathrm{H} & 2.40988400 & -3.03833000 & -0.87525400 \\ \mathrm{H} & -6.01277700 & -2.20583500 & -0.79684200 \\ \mathrm{H} & -5.01226100 & -0.06272200 & -1.26625100 \\ \mathrm{H} & -4.14723200 & -2.07978500 & -2.30374800 \\ \mathrm{H} & -3.78024100 & 0.22450500 & 1.48605900 \\ \mathrm{H} & -2.04052500 & -0.80007700 & 3.24368400 \\ \mathrm{H} & -1.35228500 & -3.26438900 & 2.38837500 \\ \mathrm{H} & -2.66590400 & -3.75019100 & 0.10093900 \\ \mathrm{H} & -2.84708800 & 2.88731500 & -0.62645900 \\ \mathrm{H} & -1.51733400 & 2.83741600 & -2.95136100 \\ \mathrm{H} & 1.06678500 & 3.43113300 & -2.44596100 \\ \mathrm{H} & 1.31822000 & 3.85196200 & 0.18830300 \\ \mathrm{H} & -2.45668000 & 2.80330900 & 2.47890000 \\ \mathrm{H} & -0.11703800 & 3.26363400 & 2.95140500 \\ \mathrm{H} & -1.62303900 & 5.05621300 & 2.41085000 \\ \mathrm{H} & 6.52886600 & -0.87846300 & -0.48278100 \\ \mathrm{H} & 4.81742600 & -2.04543100 & -1.69604000 \\ \mathrm{H} & 4.97206700 & 0.37863300 & -1.81101600 \\ \mathrm{H} & 3.50857100 & -2.77619000 & 1.11291900 \\ \mathrm{H} & 2.13442900 & -1.73108100 & 3.15541000 \\ & 2.28113700 & 0.95333200 & 2.97077900 \\ \mathrm{H} & 3.77435500 & 1.55228900 & 0.82541100 \\ \mathrm{H} & 2.02591300 & -1.88369800 & -2.23619400\end{array}$

$\begin{array}{cccc}\text { TS9 } & & & \\ \text { CS } & & & \\ \mathrm{Ti} & -0.22523100 & 1.30568400 & -0.54107400 \\ \mathrm{Ti} & 1.57367000 & -0.33304000 & 0.45892900 \\ \mathrm{Ti} & -1.10612000 & -1.14646500 & 0.22098200 \\ \mathrm{Si} & -1.65672000 & 3.47883900 & 2.11932200 \\ \mathrm{Si} & 5.10288300 & -0.44858200 & -0.96151400 \\ \mathrm{Si} & -4.70335200 & -1.22117400 & -0.87768100 \\ \mathrm{C} & 1.60274900 & 0.41126900 & -1.46950300 \\ \mathrm{C} & 0.56741800 & 0.31595900 & -2.42094300 \\ \mathrm{C} & -0.61853500 & -0.48940400 & -2.26063900 \\ \mathrm{C} & -0.80615400 & -1.85909400 & -1.81517300\end{array}$




\begin{tabular}{|c|c|c|c|}
\hline $\mathrm{C}$ & 0.22923100 & -2.75833900 & -1.37404800 \\
\hline $\mathrm{C}$ & 1.59247200 & -2.59467400 & -1.40585800 \\
\hline $\mathrm{C}$ & -1.12159600 & 3.32872200 & 0.34029000 \\
\hline $\mathrm{C}$ & 0.16946500 & 3.63846600 & -0.19645500 \\
\hline $\mathrm{C}$ & 0.14640400 & 3.45013100 & -1.59791200 \\
\hline $\mathrm{C}$ & -1.16053300 & 3.02738600 & -1.95994600 \\
\hline $\mathrm{C}$ & -1.93608500 & 2.95966100 & -0.77753600 \\
\hline $\mathrm{C}$ & 3.99420800 & -0.46832000 & 0.53633000 \\
\hline $\mathrm{C}$ & 3.56457100 & 0.65895000 & 1.30071300 \\
\hline $\mathrm{C}$ & 2.82553800 & 0.20936600 & 2.42973100 \\
\hline $\mathrm{C}$ & 2.78248400 & -1.20265000 & 2.37877800 \\
\hline $\mathrm{C}$ & 3.47645500 & -1.61682600 & 1.21340200 \\
\hline $\mathrm{C}$ & -3.08187400 & -1.02365300 & 1.57513100 \\
\hline $\mathrm{C}$ & -2.13070500 & -1.76629400 & 2.30050600 \\
\hline $\mathrm{C}$ & -1.87841800 & -2.97004800 & 1.58191100 \\
\hline $\mathrm{C}$ & -2.69218300 & -2.96657700 & 0.42499900 \\
\hline $\mathrm{C}$ & -3.43668000 & -1.74913200 & 0.38983800 \\
\hline $\mathrm{H}$ & -0.20056300 & 0.27130600 & 1.01137800 \\
\hline $\mathrm{H}$ & 1.18045200 & 1.43858800 & 0.58446000 \\
\hline $\mathrm{H}$ & 0.39302200 & -1.59359400 & 1.12882100 \\
\hline $\mathrm{H}$ & -1.78980300 & 0.46778600 & -0.15420100 \\
\hline $\mathrm{H}$ & 2.41305000 & 1.08679500 & -1.76511300 \\
\hline $\mathrm{H}$ & 0.58243400 & 0.94588300 & -3.31479000 \\
\hline $\mathrm{H}$ & -1.46086800 & -0.14414200 & -2.86312400 \\
\hline $\mathrm{H}$ & -1.72930900 & -2.33224400 & -2.14320400 \\
\hline $\mathrm{H}$ & -0.13522000 & -3.68799700 & -0.93113500 \\
\hline $\mathrm{H}$ & 2.22445500 & -3.37007200 & -0.98491700 \\
\hline $\mathrm{H}$ & -5.98268700 & -1.96192600 & -0.69942100 \\
\hline $\mathrm{H}$ & -4.97732200 & 0.23156800 & -0.72657100 \\
\hline $\mathrm{H}$ & -4.21839300 & -1.49083500 & -2.25919500 \\
\hline $\mathrm{H}$ & -3.45629000 & -0.04470700 & 1.84707800 \\
\hline $\mathrm{H}$ & -1.64598100 & -1.45897300 & 3.21849100 \\
\hline $\mathrm{H}$ & -1.18306700 & -3.74795700 & 1.87278800 \\
\hline $\mathrm{H}$ & -2.72476700 & -3.75029200 & -0.32282400 \\
\hline $\mathrm{H}$ & -2.97489200 & 2.65753700 & -0.72548400 \\
\hline $\mathrm{H}$ & -1.50051600 & 2.78686900 & -2.95979800 \\
\hline $\mathrm{H}$ & 0.98593200 & 3.57573900 & -2.27019100 \\
\hline $\mathrm{H}$ & 1.03042700 & 3.95142100 & 0.38099100 \\
\hline $\mathrm{H}$ & -2.74418800 & 2.50762500 & 2.41616200 \\
\hline $\mathrm{H}$ & -0.50689900 & 3.23008300 & 3.02875200 \\
\hline $\mathrm{H}$ & -2.18288800 & 4.84174200 & 2.41776300 \\
\hline $\mathrm{H}$ & 6.53211500 & -0.61624900 & -0.57122100 \\
\hline $\mathrm{H}$ & 4.75856200 & -1.55969600 & -1.88778000 \\
\hline $\mathrm{H}$ & 4.98146300 & 0.85321100 & -1.66971400 \\
\hline $\mathrm{H}$ & 3.60518100 & -2.64438100 & 0.89602700 \\
\hline $\mathrm{H}$ & 2.27311300 & -1.85239400 & 3.07943700 \\
\hline
\end{tabular}




\begin{tabular}{|c|c|c|c|}
\hline $\mathrm{H}$ & 2.35911200 & 0.83960500 & 3.17672000 \\
\hline $\mathrm{H}$ & 3.76347200 & 1.69588100 & 1.05786200 \\
\hline $\mathrm{H}$ & 2.07110300 & -1.83864300 & -2.00591900 \\
\hline \multicolumn{4}{|c|}{$\mathrm{B} 2^{\mathrm{CS}}$} \\
\hline $\mathrm{Ti}$ & -0.10044800 & 1.48025400 & -0.57574100 \\
\hline $\mathrm{Ti}$ & 1.53944800 & -0.42630600 & 0.43508500 \\
\hline $\mathrm{Ti}$ & -1.08078400 & -1.02117100 & 0.24200100 \\
\hline $\mathrm{Si}$ & -1.48850100 & 3.27970700 & 2.36964900 \\
\hline $\mathrm{Si}$ & 4.87310300 & -1.33764200 & -1.12232600 \\
\hline $\mathrm{Si}$ & -4.49368400 & -2.06649000 & -1.15122400 \\
\hline $\mathrm{C}$ & 1.80079200 & 0.53092800 & -1.39213900 \\
\hline $\mathrm{C}$ & 0.98173900 & 0.82641000 & -2.49060900 \\
\hline $\mathrm{C}$ & -0.35995900 & 0.39904900 & -2.68672100 \\
\hline $\mathrm{C}$ & -1.06288700 & -0.79413000 & -2.30564200 \\
\hline $\mathrm{C}$ & -0.67555300 & -1.99769200 & -1.69141800 \\
\hline $\mathrm{C}$ & 0.50983200 & -2.19973100 & -0.88848800 \\
\hline $\mathrm{C}$ & -0.92466200 & 3.37294900 & 0.59450900 \\
\hline $\mathrm{C}$ & 0.38875900 & 3.69738800 & 0.13042000 \\
\hline $\mathrm{C}$ & 0.38494400 & 3.73042300 & -1.28504600 \\
\hline $\mathrm{C}$ & -0.93499600 & 3.43955300 & -1.72323400 \\
\hline $\mathrm{C}$ & -1.73412900 & 3.22187400 & -0.57676200 \\
\hline $\mathrm{C}$ & 3.87109800 & -1.05412200 & 0.42702200 \\
\hline $\mathrm{C}$ & 3.74518100 & 0.16286000 & 1.16261100 \\
\hline $\mathrm{C}$ & 2.98030500 & -0.07346000 & 2.33577000 \\
\hline $\mathrm{C}$ & 2.61990400 & -1.44149000 & 2.34696600 \\
\hline $\mathrm{C}$ & 3.14271500 & -2.03707400 & 1.17094900 \\
\hline $\mathrm{C}$ & -3.26857500 & -0.86104600 & 1.23465800 \\
\hline $\mathrm{C}$ & -2.35420800 & -1.18623400 & 2.26031700 \\
\hline $\mathrm{C}$ & -1.84219500 & -2.48611400 & 1.98633700 \\
\hline $\mathrm{C}$ & -2.43810400 & -2.94816300 & 0.79144100 \\
\hline $\mathrm{C}$ & -3.33041300 & -1.94270600 & 0.29965300 \\
\hline $\mathrm{H}$ & -0.12104900 & 0.38598800 & 0.89865900 \\
\hline $\mathrm{H}$ & 1.29349000 & 1.40268400 & 0.60363300 \\
\hline $\mathrm{H}$ & 0.24818000 & -1.32003600 & 1.38235200 \\
\hline $\mathrm{H}$ & -1.67776200 & 0.64340600 & -0.19598600 \\
\hline $\mathrm{H}$ & 2.73620000 & 1.09925500 & -1.40862400 \\
\hline $\mathrm{H}$ & 1.31048100 & 1.59009200 & -3.19958500 \\
\hline $\mathrm{H}$ & -0.91499900 & 0.99624100 & -3.40835900 \\
\hline $\mathrm{H}$ & -2.07949100 & -0.81246800 & -2.69781500 \\
\hline $\mathrm{H}$ & -1.36864000 & -2.82456500 & -1.81864300 \\
\hline $\mathrm{H}$ & 0.61972500 & -3.20689500 & -0.48810400 \\
\hline $\mathrm{H}$ & -5.72944600 & -2.81617100 & -0.79219800 \\
\hline $\mathrm{H}$ & -4.89167200 & -0.70380700 & -1.59549100 \\
\hline $\mathrm{H}$ & -3.85199000 & -2.78383300 & -2.28761400 \\
\hline $\mathrm{H}$ & -3.81661200 & 0.06941900 & 1.15525900 \\
\hline
\end{tabular}




$\begin{array}{lrrr}\mathrm{H} & -2.07377200 & -0.55195400 & 3.09158900 \\ \mathrm{H} & -1.11593700 & -3.02196900 & 2.58387000 \\ \mathrm{H} & -2.24061500 & -3.90461800 & 0.32157100 \\ \mathrm{H} & -2.78537900 & 2.96155500 & -0.58434900 \\ \mathrm{H} & -1.26791000 & 3.38239600 & -2.75144200 \\ \mathrm{H} & 1.24011000 & 3.92982000 & -1.91840900 \\ \mathrm{H} & 1.25105900 & 3.87800400 & 0.75963500 \\ \mathrm{H} & -2.64756800 & 2.35744300 & 2.49810600 \\ \mathrm{H} & -0.37960100 & 2.81502300 & 3.24414100 \\ \mathrm{H} & -1.92298400 & 4.61799400 & 2.86289600 \\ \mathrm{H} & 6.23063300 & -1.85283900 & -0.78352900 \\ \mathrm{H} & 4.21339700 & -2.34527900 & -1.99378100 \\ \mathrm{H} & 5.04792100 & -0.05964700 & -1.85999800 \\ \mathrm{H} & 3.01448600 & -3.07482100 & 0.88476500 \\ \mathrm{H} & 2.02031800 & -1.93775400 & 3.09895600 \\ \mathrm{H} & 2.70345100 & 0.66801800 & 3.07524200 \\ \mathrm{H} & 4.14833700 & 1.12326200 & 0.86339200 \\ \mathrm{H} & 1.45652000 & -1.83994900 & -1.31867500\end{array}$

\section{TS10 ${ }^{\mathrm{CS}}$}

Ti

Ti

$\mathrm{Ti}$

$\mathrm{Si}$

$\mathrm{Si}$

$\mathrm{Si}$

C

C

C

C

C

C

C

C

C

C

C

C

C

C

C

C

C

C

C

C

$\begin{array}{ccc}-0.23888100 & 1.40903000 & -0.58351900 \\ 1.51292900 & -0.45749300 & 0.33391000 \\ -1.10410800 & -1.12978500 & 0.22085300 \\ 0.02309100 & 3.39767000 & 2.54927900 \\ 4.68938000 & -1.61898900 & -1.34196100 \\ -4.82939600 & -1.20704800 & -0.49687800 \\ 1.70247400 & 0.55114800 & -1.45713800 \\ 0.90658500 & 0.90857300 & -2.56510800 \\ -0.46988200 & 0.66579500 & -2.74684300 \\ -1.35449000 & -0.30728900 & -2.14808700 \\ -1.13092800 & -1.71314400 & -1.90882800 \\ 0.10849400 & -2.30328800 & -1.58272400 \\ -0.43662800 & 3.37057900 & 0.74119100 \\ 0.41409900 & 3.69802800 & -0.36787900 \\ -0.34508800 & 3.64629700 & -1.55671300 \\ -1.67036500 & 3.26717200 & -1.21489300 \\ -1.72883000 & 3.11555600 & 0.19142100 \\ 3.81503600 & -1.18741300 & 0.24908300 \\ 3.80125000 & 0.08170200 & 0.90231700 \\ 3.08743100 & -0.03014600 & 2.12195100 \\ 2.65681900 & -1.37420300 & 2.25276000 \\ 3.08654500 & -2.08097200 & 1.10300700 \\ -2.94382100 & -1.08969300 & 1.75991900 \\ -1.92567400 & -1.86432600 & 2.35525700 \\ -1.74439800 & -3.03165600 & 1.56109200 \\ -2.66555600 & -2.97321300 & 0.49054300\end{array}$




$\begin{array}{lrrc}\mathrm{C} & -3.41685000 & -1.75953800 & 0.58455600 \\ \mathrm{H} & 0.13087500 & -0.82641800 & 1.42477600 \\ \mathrm{H} & 0.90995300 & 1.16591500 & 0.79592800 \\ \mathrm{H} & 0.45915900 & -2.05337300 & -0.05292400 \\ \mathrm{H} & -1.49080600 & 0.59879900 & 0.41924700 \\ \mathrm{H} & 2.67229100 & 1.05888300 & -1.49725900 \\ \mathrm{H} & 1.30891900 & 1.64908600 & -3.26087200 \\ \mathrm{H} & -0.95910500 & 1.33128300 & -3.45672700 \\ \mathrm{H} & -2.39865600 & -0.09286700 & -2.36921700 \\ \mathrm{H} & -1.99644300 & -2.35921000 & -2.00533900 \\ \mathrm{H} & 0.14344800 & -3.38703800 & -1.50298300 \\ \mathrm{H} & -6.10453000 & -1.88262800 & -0.12699500 \\ \mathrm{H} & -5.02662500 & 0.26034600 & -0.35759700 \\ \mathrm{H} & -4.55716300 & -1.53074800 & -1.92578500 \\ \mathrm{H} & -3.29963400 & -0.13392900 & 2.12366300 \\ \mathrm{H} & -1.36906900 & -1.60673200 & 3.24639300 \\ \mathrm{H} & -1.02882000 & -3.82318100 & 1.74739100 \\ \mathrm{H} & -2.77673800 & -3.72720500 & -0.28008100 \\ \mathrm{H} & -2.60830000 & 2.83167200 & 0.75525600 \\ \mathrm{H} & -2.49114400 & 3.11670900 & -1.90567900 \\ \mathrm{H} & 0.02734800 & 3.82349000 & -2.55750900 \\ \mathrm{H} & 1.47045400 & 3.92927500 & -0.30245000 \\ \mathrm{H} & -0.69567900 & 2.33537700 & 3.29965900 \\ \mathrm{H} & 1.49045000 & 3.22440800 & 2.70843300 \\ \mathrm{H} & -0.34359600 & 4.70649300 & 3.16433800 \\ \mathrm{H} & 6.02642700 & -2.22148000 & -1.07356600 \\ \mathrm{H} & 3.90198400 & -2.61659200 & -2.11717300 \\ \mathrm{H} & 4.89551300 & -0.39611900 & -2.16051300 \\ \mathrm{H} & 2.90597900 & -3.13220300 & 0.90807300 \\ \mathrm{H} & 2.06730500 & -1.77904200 & 3.06543600 \\ \mathrm{H} & 2.89081300 & 0.77485200 & 2.81897800 \\ & 4.23515800 & 0.99526900 & 0.51327400 \\ \mathrm{H} & 1.03117400 & -1.85430500 & -1.94101000\end{array}$

\begin{tabular}{cccc}
$\mathbf{B 3}^{\mathbf{C S}}$ & & \\
$\mathrm{Ti}$ & -0.28986800 & 1.26523000 & -0.62291000 \\
$\mathrm{Ti}$ & 1.62575000 & -0.32112500 & 0.40253000 \\
$\mathrm{Ti}$ & -1.03904000 & -1.32910600 & 0.08060600 \\
$\mathrm{Si}$ & -0.62594300 & 3.24861500 & 2.51254600 \\
$\mathrm{Si}$ & 5.02716600 & -1.13942100 & -1.09415100 \\
$\mathrm{Si}$ & -4.77243800 & -0.90789500 & -0.15535000 \\
$\mathrm{C}$ & 1.75947000 & 0.56896400 & -1.41238800 \\
$\mathrm{C}$ & 0.98206000 & 0.80487100 & -2.57831600 \\
$\mathrm{C}$ & -0.36443800 & 0.49913100 & -2.80566100 \\
$\mathrm{C}$ & -1.27268900 & -0.38205500 & -2.08247000 \\
$\mathrm{C}$ & -1.13638400 & -1.84246900 & -1.91707500 \\
& & \multicolumn{2}{c}{$\mathrm{S} 56$} \\
\end{tabular}




\begin{tabular}{|c|c|c|c|}
\hline $\mathrm{C}$ & 0.23095200 & -2.46641800 & -1.95031100 \\
\hline $\mathrm{C}$ & -0.87042800 & 3.18224600 & 0.66558400 \\
\hline $\mathrm{C}$ & 0.05616300 & 3.61767500 & -0.34103800 \\
\hline $\mathrm{C}$ & -0.54964600 & 3.48335000 & -1.60715900 \\
\hline $\mathrm{C}$ & -1.85563800 & 2.95332300 & -1.41440900 \\
\hline $\mathrm{C}$ & -2.05548800 & 2.78413000 & -0.02567200 \\
\hline $\mathrm{C}$ & 4.02309800 & -0.75358400 & 0.43085700 \\
\hline $\mathrm{C}$ & 3.81072000 & 0.52803400 & 1.02243200 \\
\hline $\mathrm{C}$ & 3.06584400 & 0.37285200 & 2.21986200 \\
\hline $\mathrm{C}$ & 2.81040000 & -1.00986100 & 2.39382000 \\
\hline $\mathrm{C}$ & 3.37917800 & -1.69808100 & 1.29391200 \\
\hline $\mathrm{C}$ & -2.66519700 & -1.33081700 & 1.85940500 \\
\hline $\mathrm{C}$ & -1.69168100 & -2.28461200 & 2.20849600 \\
\hline $\mathrm{C}$ & -1.72879300 & -3.32569900 & 1.23613300 \\
\hline $\mathrm{C}$ & -2.74303100 & -3.01262500 & 0.30298600 \\
\hline $\mathrm{C}$ & -3.32910600 & -1.75606700 & 0.66104000 \\
\hline $\mathrm{H}$ & 0.51257200 & -1.62746000 & 0.96861300 \\
\hline $\mathrm{H}$ & 0.81187200 & 1.19638700 & 0.83818500 \\
\hline $\mathrm{H}$ & 0.78652000 & -2.32164700 & -0.98136700 \\
\hline $\mathrm{H}$ & -1.28310300 & 0.36946000 & 0.54381700 \\
\hline $\mathrm{H}$ & 2.68443900 & 1.15400200 & -1.45921400 \\
\hline $\mathrm{H}$ & 1.39124900 & 1.50636300 & -3.30984000 \\
\hline $\mathrm{H}$ & -0.82666600 & 1.07990900 & -3.60336100 \\
\hline $\mathrm{H}$ & -2.30961400 & -0.12179000 & -2.30191400 \\
\hline $\mathrm{H}$ & -1.94368600 & -2.43654200 & -2.33766300 \\
\hline $\mathrm{H}$ & 0.17116200 & -3.54978000 & -2.08410200 \\
\hline $\mathrm{H}$ & -6.06554900 & -1.54829700 & 0.21672500 \\
\hline $\mathrm{H}$ & -4.82673800 & 0.51534100 & 0.27281900 \\
\hline $\mathrm{H}$ & -4.66317300 & -0.96769800 & -1.63956300 \\
\hline $\mathrm{H}$ & -2.86058800 & -0.40832600 & 2.39125100 \\
\hline $\mathrm{H}$ & -1.00730300 & -2.21825400 & 3.04431200 \\
\hline $\mathrm{H}$ & -1.09100900 & -4.20188400 & 1.21850700 \\
\hline $\mathrm{H}$ & -3.02794700 & -3.62374700 & -0.54360300 \\
\hline $\mathrm{H}$ & -2.95266100 & 2.39063400 & 0.43514000 \\
\hline $\mathrm{H}$ & -2.56966500 & 2.71517900 & -2.19346300 \\
\hline $\mathrm{H}$ & -0.08832000 & 3.70676600 & -2.56084500 \\
\hline $\mathrm{H}$ & 1.06371700 & 3.97044500 & -0.15647700 \\
\hline $\mathrm{H}$ & -1.29989700 & 2.10598900 & 3.18359000 \\
\hline $\mathrm{H}$ & 0.82359000 & 3.23903700 & 2.84191800 \\
\hline $\mathrm{H}$ & -1.20495400 & 4.50118700 & 3.07952800 \\
\hline $\mathrm{H}$ & 6.44312100 & -1.44356900 & -0.74038400 \\
\hline $\mathrm{H}$ & 4.47421000 & -2.33357300 & -1.78868000 \\
\hline $\mathrm{H}$ & 5.03402600 & 0.02410100 & -2.01882300 \\
\hline $\mathrm{H}$ & 3.33753300 & -2.77029900 & 1.13592700 \\
\hline $\mathrm{H}$ & 2.23970300 & -1.45788600 & 3.19800500 \\
\hline $\mathrm{H}$ & 2.74249600 & 1.17193100 & 2.87508800 \\
\hline
\end{tabular}




$\begin{array}{rrrr}\mathrm{H} & 4.13964000 & 1.47492400 & 0.61031000 \\ \mathrm{H} & 0.89932000 & -2.03276100 & -2.70220300\end{array}$

\section{TS11 $^{\text {CS }}$}

Ti

Ti

Ti

$\mathrm{Si}$

$\mathrm{Si}$

$\mathrm{Si}$

C

C

C

C

C

C

C

C

C

C

C

C

C

C

C

C

C

C

C

C

C

$\mathrm{H}$

$\mathrm{H}$

$\mathrm{H}$

$\mathrm{H}$

$\mathrm{H}$

$\mathrm{H}$

$\mathrm{H}$

$\mathrm{H}$

$\mathrm{H}$

$\mathrm{H}$

$\mathrm{H}$

$\mathrm{H}$

$\mathrm{H}$

$\mathrm{H}$

$\mathrm{H}$

$\begin{array}{ccc}-0.30355000 & 1.23547400 & -0.63814300 \\ 1.56996400 & -0.56069200 & 0.31631700 \\ -1.18220700 & -1.24036800 & 0.09652600 \\ -0.53024700 & 3.05745000 & 2.61091400 \\ 5.17468500 & -0.64939500 & -0.85775700 \\ -4.83935300 & -0.40255300 & -0.22596600 \\ 1.80215200 & 0.63562900 & -1.28361000 \\ 1.08541600 & 0.88231800 & -2.48868800 \\ -0.19915100 & 0.41760200 & -2.82539300 \\ -1.00936100 & -0.61162000 & -2.24012400 \\ -0.64419200 & -1.97598900 & -1.80719200 \\ 0.78957700 & -2.45421600 & -1.96587900 \\ -0.70915000 & 3.12557000 & 0.75626100 \\ 0.29926900 & 3.49483100 & -0.19093300 \\ -0.27110100 & 3.49765500 & -1.48646400 \\ -1.63519200 & 3.11796600 & -1.36805500 \\ -1.90384100 & 2.90469900 & 0.00581900 \\ 3.96630800 & -0.71128100 & 0.56294500 \\ 3.51758600 & 0.38388100 & 1.36608500 \\ 2.69011400 & -0.10990100 & 2.40447300 \\ 2.61532500 & -1.51906700 & 2.26771700 \\ 3.37929100 & -1.88767900 & 1.13261100 \\ -2.87061700 & -1.36768200 & 1.75562100 \\ -2.03392500 & -2.48371400 & 1.97266900 \\ -2.16387300 & -3.35431400 & 0.85472700 \\ -3.09265400 & -2.77740900 & -0.03627300 \\ -3.54032300 & -1.52958200 & 0.49500900 \\ 0.34865300 & -1.83107200 & 0.84750100 \\ 0.23291500 & 0.48308600 & 0.93907800 \\ 1.56308000 & -1.84845600 & -1.45327900 \\ -1.73765100 & 0.45021200 & 0.07576300 \\ 2.67613700 & 1.28876400 & -1.20561500 \\ 1.45426800 & 1.66759300 & -3.15100600 \\ -0.69716400 & 0.99652900 & -3.60342400 \\ -2.04484800 & -0.53523600 & -2.57456600 \\ -1.34440900 & -2.72238800 & -2.19102300 \\ 0.89875600 & -3.48289700 & -1.61063600 \\ -6.20975300 & -0.94029500 & 0.00676300 \\ -4.76130500 & 0.93629800 & 0.41449600 \\ -4.66558400 & -0.25799900 & -1.69786400 \\ -2.97865000 & -0.51808400 & 2.41904400 \\ -1.38424900 & -2.63760400 & 2.82540600\end{array}$




$\begin{array}{crrc}\mathrm{H} & -1.62522300 & -4.28214900 & 0.70359700 \\ \mathrm{H} & -3.39333200 & -3.19951000 & -0.98793800 \\ \mathrm{H} & -2.85771300 & 2.60232400 & 0.41808300 \\ \mathrm{H} & -2.34434500 & 3.01212300 & -2.18011800 \\ \mathrm{H} & 0.24724500 & 3.72799300 & -2.40808200 \\ \mathrm{H} & 1.33121400 & 3.72339500 & 0.04518200 \\ \mathrm{H} & -1.38625500 & 1.98813600 & 3.18955700 \\ \mathrm{H} & 0.88875000 & 2.81863400 & 2.98475400 \\ \mathrm{H} & -0.94679000 & 4.34718100 & 3.23319400 \\ \mathrm{H} & 6.56563100 & -0.89849200 & -0.38214300 \\ \mathrm{H} & 4.85094600 & -1.69607500 & -1.86397700 \\ \mathrm{H} & 5.15248100 & 0.69165000 & -1.49852200 \\ \mathrm{H} & 3.50837900 & -2.89919400 & 0.76270100 \\ \mathrm{H} & 2.03980400 & -2.19057300 & 2.89207000 \\ \mathrm{H} & 2.19058000 & 0.48821300 & 3.15608900 \\ \mathrm{H} & 3.75400000 & 1.42766400 & 1.19735400 \\ \mathrm{H} & 1.12071200 & -2.41011200 & -3.01446100\end{array}$

$\begin{array}{cccc}\text { B4 }^{\text {CS }} & & & \\ \mathrm{Ti} & -0.28218000 & 1.24472600 & -0.65346700 \\ \mathrm{Ti} & 1.56910500 & -0.57831100 & 0.30070500 \\ \mathrm{Ti} & -1.17783600 & -1.22573000 & 0.07349400 \\ \mathrm{Si} & -0.73737000 & 2.92681100 & 2.65022100 \\ \mathrm{Si} & 5.20033200 & -0.59497600 & -0.78227700 \\ \mathrm{Si} & -4.83140700 & -0.39982500 & -0.27095400 \\ \mathrm{C} & 1.82020600 & 0.62678900 & -1.28742200 \\ \mathrm{C} & 1.10674900 & 0.89979800 & -2.48881500 \\ \mathrm{C} & -0.17628600 & 0.43324800 & -2.84692000 \\ \mathrm{C} & -0.97782100 & -0.62254000 & -2.31557900 \\ \mathrm{C} & -0.62659800 & -1.96820000 & -1.84321100 \\ \mathrm{C} & 0.80668700 & -2.45826700 & -1.97066900 \\ \mathrm{C} & -0.74949400 & 3.08054100 & 0.79242400 \\ \mathrm{C} & 0.35007700 & 3.45587900 & -0.04363700 \\ \mathrm{C} & -0.10249300 & 3.53437200 & -1.38326500 \\ \mathrm{C} & -1.48450100 & 3.20220300 & -1.40208500 \\ \mathrm{C} & -1.88004300 & 2.93717500 & -0.06922900 \\ \mathrm{C} & 3.95633700 & -0.68630400 & 0.60519200 \\ \mathrm{C} & 3.47002600 & 0.39503500 & 1.40654000 \\ \mathrm{C} & 2.62750600 & -0.12120900 & 2.42101600 \\ \mathrm{C} & 2.58002600 & -1.53044600 & 2.27153700 \\ \mathrm{C} & 3.37609300 & -1.87720500 & 1.15157900 \\ \mathrm{C} & -2.87061800 & -1.35164400 & 1.72635700 \\ \mathrm{C} & -2.03131600 & -2.46392000 & 1.95084100 \\ \mathrm{C} & -2.15215200 & -3.33867900 & 0.83474200 \\ \mathrm{C} & -3.07761900 & -2.76805400 & -0.06319000 \\ \mathrm{C} & -3.53119300 & -1.51913600 & 0.46191300\end{array}$




$\begin{array}{lrrc}\mathrm{H} & 0.34571000 & -1.86597400 & 0.79385500 \\ \mathrm{H} & 0.10462400 & 0.33453000 & 0.88836000 \\ \mathrm{H} & 1.57593500 & -1.80740100 & -1.50979300 \\ \mathrm{H} & -1.77168500 & 0.44891100 & -0.05023200 \\ \mathrm{H} & 2.69219500 & 1.27989900 & -1.19266600 \\ \mathrm{H} & 1.47457400 & 1.69950200 & -3.13362100 \\ \mathrm{H} & -0.67664200 & 1.03614900 & -3.60495900 \\ \mathrm{H} & -2.01418800 & -0.54190300 & -2.64604600 \\ \mathrm{H} & -1.32962000 & -2.72003600 & -2.21087400 \\ \mathrm{H} & 0.91958600 & -3.46083000 & -1.54886700 \\ \mathrm{H} & -6.20025700 & -0.93932800 & -0.03374000 \\ \mathrm{H} & -4.75632200 & 0.94529900 & 0.35591500 \\ \mathrm{H} & -4.65537100 & -0.27132400 & -1.74395100 \\ \mathrm{H} & -2.98325900 & -0.49940100 & 2.38556500 \\ \mathrm{H} & -1.38459100 & -2.61237800 & 2.80678200 \\ \mathrm{H} & -1.60933100 & -4.26506800 & 0.68998200 \\ \mathrm{H} & -3.37300200 & -3.19434600 & -1.01460000 \\ \mathrm{H} & -2.87542100 & 2.64933500 & 0.24390600 \\ \mathrm{H} & -2.12217900 & 3.16167100 & -2.27683600 \\ \mathrm{H} & 0.50250700 & 3.78975600 & -2.24333000 \\ \mathrm{H} & 1.36310200 & 3.63937700 & 0.29211800 \\ \mathrm{H} & -1.67492600 & 1.86560000 & 3.10432200 \\ \mathrm{H} & 0.63434200 & 2.61924200 & 3.13403700 \\ \mathrm{H} & -1.16451000 & 4.20249200 & 3.29355400 \\ \mathrm{H} & 6.58225100 & -0.82669500 & -0.27249900 \\ \mathrm{H} & 4.91914900 & -1.63808800 & -1.80493800 \\ \mathrm{H} & 5.17509800 & 0.75055700 & -1.41360200 \\ \mathrm{H} & 3.53155900 & -2.88332500 & 0.77709300 \\ \mathrm{H} & 2.00083800 & -2.21648300 & 2.87646300 \\ \mathrm{H} & 2.09914000 & 0.46197700 & 3.16469600 \\ & 3.69443400 & 1.44378800 & 1.25278400 \\ & 1.13309900 & -2.48532800 & -3.02158400 \\ & & & \\ \mathrm{H} & & & \\ \mathrm{H} & & \end{array}$

\section{$\mathrm{TS}_{12}{ }^{\mathrm{CS}}$}

$\mathrm{Ti}$

Ti

$\mathrm{Ti}$

$\mathrm{Si}$

$\mathrm{Si}$

$\mathrm{Si}$

C

C

C

C

C

C

$\begin{array}{ccc}-0.27509700 & 1.24264000 & -0.69558300 \\ 1.55088900 & -0.54111000 & 0.36703600 \\ -1.16539000 & -1.13941200 & 0.22742800 \\ -1.13167700 & 3.22362200 & 2.31422100 \\ 5.14313400 & -0.70340500 & -0.86338400 \\ -4.80107600 & -0.66286700 & -0.69538500 \\ 1.67279900 & 0.38512100 & -1.41621000 \\ 0.72683600 & 0.43054300 & -2.48849400 \\ -0.48969700 & -0.33338100 & -2.60402000 \\ -0.91365900 & -1.60475200 & -2.14403200 \\ -0.27954300 & -2.57741500 & -1.33641200 \\ 1.21061600 & -2.80331200 & -1.24043900\end{array}$




\begin{tabular}{|c|c|c|c|}
\hline $\mathrm{C}$ & -0.78601300 & 3.21122200 & 0.48301900 \\
\hline $\mathrm{C}$ & 0.48543700 & 3.41624600 & -0.15033000 \\
\hline $\mathrm{C}$ & 0.29699900 & 3.44370600 & -1.55351700 \\
\hline $\mathrm{C}$ & -1.08360100 & 3.25618500 & -1.81511100 \\
\hline $\mathrm{C}$ & -1.74839900 & 3.11540900 & -0.57387000 \\
\hline $\mathrm{C}$ & 3.96148100 & -0.53153900 & 0.56799600 \\
\hline $\mathrm{C}$ & 3.46409900 & 0.68268100 & 1.13872100 \\
\hline $\mathrm{C}$ & 2.67780500 & 0.37712100 & 2.27684900 \\
\hline $\mathrm{C}$ & 2.67419800 & -1.03284100 & 2.43520400 \\
\hline $\mathrm{C}$ & 3.44514600 & -1.58922200 & 1.38519700 \\
\hline $\mathrm{C}$ & -3.03747900 & -0.90348100 & 1.66280900 \\
\hline $\mathrm{C}$ & -2.17928500 & -1.84668400 & 2.26828700 \\
\hline $\mathrm{C}$ & -2.14176200 & -2.99744400 & 1.43260200 \\
\hline $\mathrm{C}$ & -3.00026900 & -2.76561200 & 0.33274500 \\
\hline $\mathrm{C}$ & -3.55898900 & -1.46082900 & 0.44528600 \\
\hline $\mathrm{H}$ & 0.30595300 & -1.66462100 & 1.12178600 \\
\hline $\mathrm{H}$ & 0.01744700 & 0.37482800 & 0.91282600 \\
\hline $\mathrm{H}$ & 1.82503500 & -1.87503700 & -1.20692100 \\
\hline $\mathrm{H}$ & -1.82102500 & 0.45973500 & -0.25685500 \\
\hline $\mathrm{H}$ & 2.54106100 & 1.02307500 & -1.60772200 \\
\hline $\mathrm{H}$ & 0.88589700 & 1.12573100 & -3.31603900 \\
\hline $\mathrm{H}$ & -1.21414100 & 0.11903100 & -3.28630700 \\
\hline $\mathrm{H}$ & -1.93147100 & -1.85005700 & -2.44923000 \\
\hline $\mathrm{H}$ & -0.84221000 & -3.50637300 & -1.25727200 \\
\hline $\mathrm{H}$ & 1.46109200 & -3.46358600 & -0.40632600 \\
\hline $\mathrm{H}$ & -6.17884200 & -1.16561900 & -0.42974100 \\
\hline $\mathrm{H}$ & -4.80484300 & 0.80964100 & -0.49743600 \\
\hline $\mathrm{H}$ & -4.48499900 & -0.97128900 & -2.11784100 \\
\hline $\mathrm{H}$ & -3.25467900 & 0.08710600 & 2.04228300 \\
\hline $\mathrm{H}$ & -1.62213400 & -1.70843800 & 3.18636100 \\
\hline $\mathrm{H}$ & -1.54831500 & -3.88749800 & 1.60322300 \\
\hline $\mathrm{H}$ & -3.17374900 & -3.45651200 & -0.48447300 \\
\hline $\mathrm{H}$ & -2.80993400 & 2.94308700 & -0.44466800 \\
\hline $\mathrm{H}$ & -1.54463900 & 3.19745700 & -2.79444200 \\
\hline $\mathrm{H}$ & 1.07676200 & 3.55389800 & -2.29671100 \\
\hline $\mathrm{H}$ & 1.43291800 & 3.53624300 & 0.36059900 \\
\hline $\mathrm{H}$ & -2.29258800 & 2.35328500 & 2.63908500 \\
\hline $\mathrm{H}$ & 0.06304800 & 2.76096700 & 3.06961200 \\
\hline $\mathrm{H}$ & -1.45776600 & 4.59806000 & 2.79176600 \\
\hline $\mathrm{H}$ & 6.55459000 & -0.78864300 & -0.39052800 \\
\hline $\mathrm{H}$ & 4.85381100 & -1.94650000 & -1.62871700 \\
\hline $\mathrm{H}$ & 5.04168000 & 0.47517800 & -1.76363900 \\
\hline $\mathrm{H}$ & 3.62093300 & -2.64808200 & 1.22848900 \\
\hline $\mathrm{H}$ & 2.14113900 & -1.58712400 & 3.19742600 \\
\hline $\mathrm{H}$ & 2.15454800 & 1.09075100 & 2.90095700 \\
\hline $\mathrm{H}$ & 3.64291600 & 1.67740500 & 0.74857400 \\
\hline
\end{tabular}


$\mathrm{H}$

$\mathrm{B5}^{\mathrm{CS}}$

$\mathrm{Ti}$

$\mathrm{Ti}$

$\mathrm{Ti}$

$\mathrm{Si}$

$\mathrm{Si}$

$\mathrm{Si}$

C

C

C

C

C

C

C

C

C

C

C

C

C

C

C

C

C

C

C

C

C

$\mathrm{H}$

$\mathrm{H}$

$\mathrm{H}$

$\mathrm{H}$

$\mathrm{H}$

$\mathrm{H}$

$\mathrm{H}$

$\mathrm{H}$

$\mathrm{H}$

$\mathrm{H}$

$\mathrm{H}$

$\mathrm{H}$

$\mathrm{H}$

$\mathrm{H}$

$\mathrm{H}$

$\mathrm{H}$
$1.60802200 \quad-3.26592600$

$-2.15751500$

$\begin{array}{ccc}-0.25167000 & 1.27048700 & -0.70837900 \\ 1.51869100 & -0.62555300 & 0.35681000 \\ -1.21785700 & -1.05584100 & 0.25871900 \\ -0.96447100 & 3.38360300 & 2.23793700 \\ 5.09449000 & -0.83239000 & -0.91072600 \\ -4.83029500 & -0.75561600 & -0.83307400 \\ 1.60666000 & 0.25735300 & -1.45944800 \\ 0.57733500 & 0.27648700 & -2.44633800 \\ -0.67446000 & -0.46888000 & -2.33586200 \\ -0.98601200 & -1.80138500 & -1.92037500 \\ -0.22912300 & -2.70297200 & -1.15624000 \\ 1.26602600 & -2.87365300 & -1.12044900 \\ -0.61754300 & 3.29909000 & 0.40912700 \\ 0.66262700 & 3.39829300 & -0.23315000 \\ 0.47301400 & 3.39460800 & -1.63674700 \\ -0.91757300 & 3.29529900 & -1.89013100 \\ -1.58776800 & 3.23572800 & -0.64474200 \\ 3.92904500 & -0.60345800 & 0.52629100 \\ 3.43961800 & 0.63512700 & 1.04799600 \\ 2.66371400 & 0.38051400 & 2.20536800 \\ 2.66174100 & -1.02138700 & 2.42767200 \\ 3.42256900 & -1.62396800 & 1.39697400 \\ -3.15235900 & -0.69262500 & 1.59566600 \\ -2.29774600 & -1.53804700 & 2.33309400 \\ -2.20571900 & -2.77423400 & 1.63348100 \\ -3.02853900 & -2.68896200 & 0.48569500 \\ -3.61664000 & -1.39423400 & 0.42955000 \\ 0.22335600 & -1.62415600 & 1.19411600 \\ -0.00126700 & 0.36068100 & 0.88746000 \\ 1.85617900 & -1.92815600 & -1.18442200 \\ -1.82445200 & 0.58852000 & -0.18244600 \\ 2.49388000 & 0.82549300 & -1.75455800 \\ 0.68989200 & 0.86827600 & -3.35867500 \\ -1.47788900 & -0.04725700 & -2.94710200 \\ -1.99169800 & -2.12350000 & -2.18777200 \\ -0.74924200 & -3.62912100 & -0.91512800 \\ 1.57594600 & -3.48028200 & -0.26532500 \\ -6.20823600 & -1.25843900 & -0.56883800 \\ -4.86897300 & 0.72959400 & -0.79549300 \\ -4.45289600 & -1.20527600 & -2.20269800 \\ -3.40528700 & 0.32765600 & 1.85493300 \\ -1.77753400 & -1.28327400 & 3.24781100 \\ -1.60414100 & -3.62611600 & 1.92586300\end{array}$




$\begin{array}{crrc}\mathrm{H} & -3.15740900 & -3.47309800 & -0.25169500 \\ \mathrm{H} & -2.65845700 & 3.14283900 & -0.50967300 \\ \mathrm{H} & -1.38358100 & 3.23352800 & -2.86710800 \\ \mathrm{H} & 1.25593000 & 3.42053300 & -2.38441000 \\ \mathrm{H} & 1.61798500 & 3.46869600 & 0.27214100 \\ \mathrm{H} & -2.19683000 & 2.62276900 & 2.57508600 \\ \mathrm{H} & 0.18368900 & 2.83535900 & 3.00905900 \\ \mathrm{H} & -1.16945200 & 4.79083400 & 2.68654000 \\ \mathrm{H} & 6.50828100 & -0.93233500 & -0.44927300 \\ \mathrm{H} & 4.77614000 & -2.08628100 & -1.64688500 \\ \mathrm{H} & 5.00149300 & 0.32713900 & -1.83618000 \\ \mathrm{H} & 3.59918200 & -2.68839900 & 1.28755600 \\ \mathrm{H} & 2.13606500 & -1.53980900 & 3.21955300 \\ \mathrm{H} & 2.14457600 & 1.12082100 & 2.80125500 \\ \mathrm{H} & 3.61836800 & 1.61079400 & 0.61302200 \\ \mathrm{H} & 1.63542600 & -3.37861200 & -2.02654200\end{array}$

\section{TS13 $^{\mathrm{CS}}$}

Ti

Ti

Ti

$\mathrm{Si}$

$\mathrm{Si}$

$\mathrm{Si}$

C

C

C

C

C

C

C

C

C

C

C

C

C

C

C

C

C

C

C

C

C

$\mathrm{H}$

$\begin{array}{ccc}0.18757500 & 1.44319200 & -0.53972900 \\ 0.99347200 & -1.26895800 & -0.00553200 \\ -1.55838500 & -0.64184700 & 0.30293300 \\ 1.38831000 & 3.15861900 & 2.49581800 \\ 4.76019900 & -0.65939600 & -0.47625400 \\ -5.04831700 & 0.54483900 & -0.59128100 \\ 1.35813000 & 0.11682200 & -1.58561700 \\ 0.29944200 & 0.36900900 & -2.53884100 \\ -1.03685200 & -0.07590800 & -2.45222900 \\ -1.62915700 & -1.18913800 & -1.75433700 \\ -0.74970700 & -2.22362400 & -1.12232800 \\ 0.39633300 & -2.79197100 & -1.98911400 \\ 0.97692900 & 3.28870400 & 0.68325200 \\ 1.87907400 & 3.13238800 & -0.41913000 \\ 1.19029700 & 3.40146600 & -1.62341700 \\ -0.15215300 & 3.71893000 & -1.30181000 \\ -0.28619800 & 3.65434900 & 0.10966800 \\ 3.39592200 & -1.56530300 & 0.41154500 \\ 2.75550500 & -1.19218500 & 1.63855100 \\ 1.88258900 & -2.22925100 & 2.03655100 \\ 1.94988700 & -3.25998900 & 1.05875400 \\ 2.88952500 & -2.85853200 & 0.08093100 \\ -3.33321600 & 0.07784100 & 1.76262200 \\ -2.64962600 & -0.97827700 & 2.40231700 \\ -2.86789000 & -2.16226000 & 1.63855800 \\ -3.68841400 & -1.82367200 & 0.53904400 \\ -3.97691600 & -0.42720800 & 0.58699200 \\ -0.24338300 & -1.32248300 & 1.28848700\end{array}$




$\begin{array}{lrrr}\mathrm{H} & 0.60331600 & 0.40626900 & 0.85493100 \\ \mathrm{H} & 1.38338900 & -2.29463600 & -1.88670900 \\ \mathrm{H} & -1.51670700 & 1.16606100 & 0.10282100 \\ \mathrm{H} & 2.34536500 & 0.42882400 & -1.93707900 \\ \mathrm{H} & 0.50556500 & 1.00786200 & -3.40226800 \\ \mathrm{H} & -1.72739800 & 0.44963700 & -3.11463700 \\ \mathrm{H} & -2.60831800 & -1.48790100 & -2.13190100 \\ \mathrm{H} & -1.33963700 & -3.06225700 & -0.74678600 \\ \mathrm{H} & 0.55389000 & -3.85152500 & -1.77255600 \\ \mathrm{H} & -4.71179900 & 1.99053900 & -0.50448200 \\ \mathrm{H} & -4.85129300 & 0.07457800 & -1.98987400 \\ \mathrm{H} & -6.49392700 & 0.38813200 & -0.26638700 \\ \mathrm{H} & -3.34638000 & 1.11045500 & 2.08973400 \\ \mathrm{H} & -2.05520200 & -0.90269300 & 3.30428800 \\ \mathrm{H} & -2.46577500 & -3.14338100 & 1.86002200 \\ \mathrm{H} & -4.01465100 & -2.50768800 & -0.23605300 \\ \mathrm{H} & -1.19843200 & 3.84303400 & 0.66268700 \\ \mathrm{H} & -0.94091200 & 3.94989800 & -2.00823900 \\ \mathrm{H} & 1.60598000 & 3.32665100 & -2.62090000 \\ \mathrm{H} & 2.91646700 & 2.82778300 & -0.34521300 \\ \mathrm{H} & 0.22077300 & 2.64604700 & 3.26164600 \\ \mathrm{H} & 2.55155900 & 2.25353900 & 2.69514700 \\ \mathrm{H} & 1.75030000 & 4.48681700 & 3.07020800 \\ \mathrm{H} & 6.08759300 & -0.95495500 & 0.13290300 \\ \mathrm{H} & 4.80507700 & -1.08835700 & -1.89982400 \\ \mathrm{H} & 4.55673200 & 0.81226900 & -0.41096900 \\ \mathrm{H} & 3.17073800 & -3.43269200 & -0.79432700 \\ \mathrm{H} & 1.38971400 & -4.18740100 & 1.06945500 \\ \mathrm{H} & 1.26182200 & -2.23142900 & 2.92252400 \\ \mathrm{H} & 2.90624200 & -0.26129300 & 2.17108500 \\ \mathrm{H} & 0.17699000 & -2.66224900 & -3.05425000\end{array}$

$\begin{array}{cccc}\text { B6 }^{\mathbf{C S}} & & & \\ \mathrm{Ti} & 0.22088000 & 1.33073600 & -0.56986700 \\ \mathrm{Ti} & 1.22624600 & -1.09780300 & 0.06996200 \\ \mathrm{Ti} & -1.47074300 & -0.71987400 & 0.21922000 \\ \mathrm{Si} & 0.03003900 & 3.67942900 & 2.32995800 \\ \mathrm{Si} & 4.91678200 & -0.23628400 & -0.25599300 \\ \mathrm{Si} & -5.08290700 & -0.13825000 & -0.76173100 \\ \mathrm{C} & 1.32135500 & 0.09243100 & -1.80232400 \\ \mathrm{C} & 0.18501100 & 0.11032400 & -2.64842100 \\ \mathrm{C} & -1.08540600 & -0.46022100 & -2.39225600 \\ \mathrm{C} & -1.44725100 & -1.64130600 & -1.63519800 \\ \mathrm{C} & -0.38853400 & -2.40411000 & -0.91261500 \\ \mathrm{C} & 0.80971700 & -2.88295000 & -1.75622400 \\ \mathrm{C} & 0.30998800 & 3.47474100 & 0.49616900\end{array}$




\begin{tabular}{|c|c|c|c|}
\hline $\mathrm{C}$ & 1.56430800 & 3.25258400 & -0.15995600 \\
\hline $\mathrm{C}$ & 1.34670600 & 3.20951000 & -1.55742000 \\
\hline $\mathrm{C}$ & -0.04422400 & 3.40723200 & -1.78968900 \\
\hline $\mathrm{C}$ & -0.67263100 & 3.58114100 & -0.53461800 \\
\hline $\mathrm{C}$ & 3.60839400 & -1.22976300 & 0.62337600 \\
\hline $\mathrm{C}$ & 2.88615600 & -0.85950800 & 1.80817400 \\
\hline $\mathrm{C}$ & 2.05431300 & -1.93238600 & 2.19619800 \\
\hline $\mathrm{C}$ & 2.23324200 & -2.98535600 & 1.25957600 \\
\hline $\mathrm{C}$ & 3.20273900 & -2.56026600 & 0.31722200 \\
\hline $\mathrm{C}$ & -3.31822200 & 0.01607300 & 1.59469800 \\
\hline $\mathrm{C}$ & -2.49815800 & -0.82021700 & 2.38200700 \\
\hline $\mathrm{C}$ & -2.55115500 & -2.13054900 & 1.82583500 \\
\hline $\mathrm{C}$ & -3.40885500 & -2.08814200 & 0.70055200 \\
\hline $\mathrm{C}$ & -3.88863900 & -0.75296900 & 0.53131000 \\
\hline $\mathrm{H}$ & -0.02834000 & -0.76866900 & 1.28344800 \\
\hline $\mathrm{H}$ & 1.37959000 & 0.66714300 & 0.59535300 \\
\hline $\mathrm{H}$ & 1.73714100 & -2.27522200 & -1.67846000 \\
\hline $\mathrm{H}$ & -1.48425900 & 1.07129200 & -0.02475600 \\
\hline $\mathrm{H}$ & 2.24876000 & 0.43975200 & -2.26577900 \\
\hline $\mathrm{H}$ & 0.23125400 & 0.69825500 & -3.57281200 \\
\hline $\mathrm{H}$ & -1.88617400 & -0.08321800 & -3.02939000 \\
\hline $\mathrm{H}$ & -2.35207400 & -2.14613400 & -1.97428700 \\
\hline $\mathrm{H}$ & -0.81556500 & -3.26186400 & -0.38658900 \\
\hline $\mathrm{H}$ & 1.08777600 & -3.90607600 & -1.49130600 \\
\hline $\mathrm{H}$ & -4.93365000 & 1.33187400 & -0.92937300 \\
\hline $\mathrm{H}$ & -4.83346100 & -0.81054700 & -2.06678300 \\
\hline $\mathrm{H}$ & -6.49599100 & -0.41717800 & -0.38031400 \\
\hline $\mathrm{H}$ & -3.46357800 & 1.07820700 & 1.74877000 \\
\hline $\mathrm{H}$ & -1.91493100 & -0.51565200 & 3.24176800 \\
\hline $\mathrm{H}$ & -2.02245300 & -3.00038000 & 2.19618800 \\
\hline $\mathrm{H}$ & -3.64378000 & -2.92839200 & 0.05771600 \\
\hline $\mathrm{H}$ & -1.73427900 & 3.72908700 & -0.37814900 \\
\hline $\mathrm{H}$ & -0.53730400 & 3.41635300 & -2.75415800 \\
\hline $\mathrm{H}$ & 2.10234200 & 3.04683700 & -2.31507300 \\
\hline $\mathrm{H}$ & 2.52000100 & 3.12427800 & 0.33303200 \\
\hline $\mathrm{H}$ & -1.35509500 & 3.27143400 & 2.68716800 \\
\hline $\mathrm{H}$ & 1.00174300 & 2.85714100 & 3.09929200 \\
\hline $\mathrm{H}$ & 0.20782100 & 5.09933600 & 2.74507800 \\
\hline $\mathrm{H}$ & 6.25606300 & -0.42221500 & 0.37096800 \\
\hline $\mathrm{H}$ & 5.01487100 & -0.67388400 & -1.67483000 \\
\hline $\mathrm{H}$ & 4.59833400 & 1.21564700 & -0.20802200 \\
\hline $\mathrm{H}$ & 3.56477100 & -3.14570800 & -0.51977400 \\
\hline $\mathrm{H}$ & 1.73233500 & -3.94584100 & 1.27551700 \\
\hline $\mathrm{H}$ & 1.38416800 & -1.94218300 & 3.04557100 \\
\hline $\mathrm{H}$ & 2.96248400 & 0.09070100 & 2.32069500 \\
\hline $\mathrm{H}$ & 0.58314800 & -2.82775000 & -2.82527900 \\
\hline
\end{tabular}




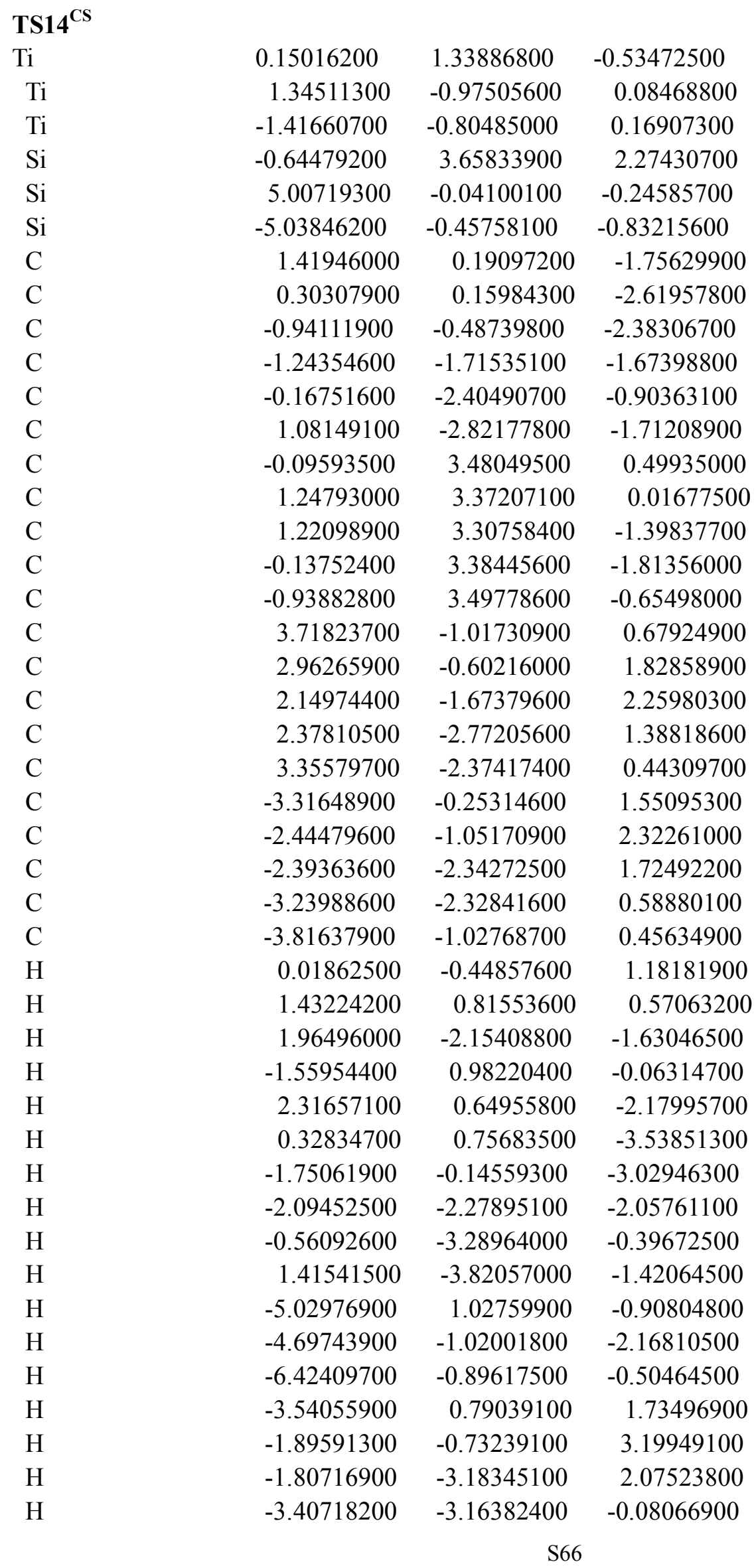




$\begin{array}{lrrc}\mathrm{H} & -2.02081200 & 3.54893000 & -0.64066700 \\ \mathrm{H} & -0.49756500 & 3.34794100 & -2.83445200 \\ \mathrm{H} & 2.08176000 & 3.21182600 & -2.04750800 \\ \mathrm{H} & 2.13930300 & 3.34252100 & 0.63090000 \\ \mathrm{H} & -2.07071900 & 3.25518100 & 2.40389900 \\ \mathrm{H} & 0.18920100 & 2.81459900 & 3.17215600 \\ \mathrm{H} & -0.52251600 & 5.06862000 & 2.73892100 \\ \mathrm{H} & 6.30688700 & -0.02053200 & 0.48361300 \\ \mathrm{H} & 5.23749000 & -0.66543700 & -1.57668700 \\ \mathrm{H} & 4.58101700 & 1.37200400 & -0.43358700 \\ \mathrm{H} & 3.74922600 & -2.99431000 & -0.35351500 \\ \mathrm{H} & 1.90085200 & -3.74302000 & 1.44616400 \\ \mathrm{H} & 1.46038300 & -1.65415300 & 3.09373700 \\ \mathrm{H} & 3.00832300 & 0.37414400 & 2.29326100 \\ \mathrm{H} & 0.87187200 & -2.80448900 & -2.78598400\end{array}$

B ${ }^{\mathrm{CS}}$

Ti

Ti

Ti

$\mathrm{Si}$

$\mathrm{Si}$

$\mathrm{Si}$

C

C

C

C

C

C

C

C

C

C

C

C

C

C

C

C

C

C

C

C

C

$\mathrm{H}$

$\begin{array}{ccc}-0.26190400 & 1.30744500 & -0.43418800 \\ 1.67086300 & -0.37599600 & 0.35195600 \\ -1.30874100 & -1.09126100 & 0.22860700 \\ -1.39238300 & 3.58106300 & 2.29602000 \\ 5.09862000 & -0.04952500 & -1.24069600 \\ -4.69433200 & -0.98209700 & -1.25233700 \\ 1.57877500 & 0.48281000 & -1.50854200 \\ 0.46760900 & 0.30435200 & -2.32957700 \\ -0.60165800 & -0.61575000 & -1.96254000 \\ -0.34240900 & -1.95074900 & -1.39393300 \\ 0.72192200 & -2.16654800 & -0.41004700 \\ 1.23695000 & -3.60702300 & -0.38825400 \\ -1.03430900 & 3.37137000 & 0.47888100 \\ 0.20480300 & 3.63052100 & -0.18995100 \\ 0.03931400 & 3.40306100 & -1.57702000 \\ -1.30373000 & 2.99986600 & -1.79700500 \\ -1.96047800 & 2.98949400 & -0.54239600 \\ 4.07968600 & -0.39787300 & 0.28072400 \\ 3.66947100 & 0.52987500 & 1.29037400 \\ 3.02997900 & -0.17541900 & 2.34110200 \\ 3.02701500 & -1.54879200 & 1.99702700 \\ 3.65843600 & -1.68502200 & 0.73497900 \\ -3.38029000 & -0.99971700 & 1.38972900 \\ -2.53897100 & -1.82196300 & 2.16570000 \\ -2.21844900 & -2.97513700 & 1.39355100 \\ -2.87553300 & -2.86203200 & 0.14393800 \\ -3.59361100 & -1.62040400 & 0.11220400 \\ -0.08750400 & 0.05966900 & 1.04716600\end{array}$




$\begin{array}{lrrr}\mathrm{H} & 1.17120900 & 1.39077900 & 0.65959300 \\ \mathrm{H} & 0.25747400 & -2.02731000 & 0.72459600 \\ \mathrm{H} & -1.82072700 & 0.61045800 & 0.11685400 \\ \mathrm{H} & 2.32202600 & 1.19363900 & -1.87993100 \\ \mathrm{H} & 0.37384300 & 0.80302100 & -3.29727600 \\ \mathrm{H} & -1.45317600 & -0.58615700 & -2.64490800 \\ \mathrm{H} & -0.75517400 & -2.82026600 & -1.91166800 \\ \mathrm{H} & 0.42761900 & -4.33765500 & -0.53471200 \\ \mathrm{H} & 1.74509400 & -3.85663000 & 0.54783100 \\ \mathrm{H} & -6.03525600 & -1.63037500 & -1.21428500 \\ \mathrm{H} & -4.88482800 & 0.48391900 & -1.09648600 \\ \mathrm{H} & -4.09821100 & -1.26660700 & -2.58597400 \\ \mathrm{H} & -3.76851000 & -0.03532500 & 1.69181800 \\ \mathrm{H} & -2.16794400 & -1.59664400 & 3.15860400 \\ \mathrm{H} & -1.58435800 & -3.79545300 & 1.70789000 \\ \mathrm{H} & -2.84129200 & -3.59349300 & -0.65303700 \\ \mathrm{H} & -2.99556900 & 2.71586700 & -0.38057200 \\ \mathrm{H} & -1.74481200 & 2.73538300 & -2.75022300 \\ \mathrm{H} & 0.81003300 & 3.49269600 & -2.33202400 \\ \mathrm{H} & 1.12598800 & 3.93659100 & 0.28989400 \\ \mathrm{H} & -2.46757000 & 2.64496400 & 2.72210100 \\ \mathrm{H} & -0.16625100 & 3.33006700 & 3.09902100 \\ \mathrm{H} & -1.85782200 & 4.96431500 & 2.60283700 \\ \mathrm{H} & 6.55523300 & -0.08721000 & -0.92345500 \\ \mathrm{H} & 4.83273500 & -1.07519100 & -2.28296700 \\ \mathrm{H} & 4.80062600 & 1.30541300 & -1.77766200 \\ \mathrm{H} & 3.80392800 & -2.61456800 & 0.20030600 \\ \mathrm{H} & 2.58779800 & -2.34939200 & 2.58152900 \\ \mathrm{H} & 2.59273400 & 0.26415500 & 3.22938200 \\ \mathrm{H} & 3.81177100 & 1.60338500 & 1.25607600 \\ \mathrm{H} & 1.95229000 & -3.74788100 & -1.20604300\end{array}$

$\begin{array}{lccc}\text { TS15 }^{\mathbf{C S}} & & & \\ \mathrm{Ti} & -0.22547800 & 1.34211100 & -0.46381300 \\ \mathrm{Ti} & 1.59263800 & -0.45984300 & 0.34617100 \\ \mathrm{Ti} & -1.29888600 & -0.98140500 & 0.28726200 \\ \mathrm{Si} & -1.09931800 & 3.73432400 & 2.26489900 \\ \mathrm{Si} & 5.05317200 & -0.21739400 & -1.22133900 \\ \mathrm{Si} & -4.67282900 & -1.20589300 & -1.28672300 \\ \mathrm{C} & 1.54042100 & 0.36775300 & -1.56694100 \\ \mathrm{C} & 0.40963700 & 0.28691200 & -2.36423100 \\ \mathrm{C} & -0.72806500 & -0.52700800 & -1.93535100 \\ \mathrm{C} & -0.46938300 & -1.89650300 & -1.42516300 \\ \mathrm{C} & 0.63192100 & -2.14995400 & -0.54605700 \\ \mathrm{C} & 1.03567900 & -3.61754000 & -0.45551700 \\ \mathrm{C} & -0.85032200 & 3.46192800 & 0.43706900\end{array}$




\begin{tabular}{|c|c|c|c|}
\hline $\mathrm{C}$ & 0.36232000 & 3.64582500 & -0.30244700 \\
\hline $\mathrm{C}$ & 0.11140600 & 3.39599100 & -1.67279500 \\
\hline $\mathrm{C}$ & -1.25969600 & 3.05328300 & -1.81041700 \\
\hline $\mathrm{C}$ & -1.84760400 & 3.10371800 & -0.52345000 \\
\hline $\mathrm{C}$ & 4.00463400 & -0.55655400 & 0.28193500 \\
\hline $\mathrm{C}$ & 3.62412900 & 0.36551800 & 1.30778200 \\
\hline $\mathrm{C}$ & 2.94940300 & -0.33553700 & 2.33822000 \\
\hline $\mathrm{C}$ & 2.90005600 & -1.70224000 & 1.96815900 \\
\hline $\mathrm{C}$ & 3.53658200 & -1.83731000 & 0.70877900 \\
\hline $\mathrm{C}$ & -3.42543000 & -0.81315100 & 1.35589200 \\
\hline $\mathrm{C}$ & -2.57284300 & -1.48645900 & 2.25567200 \\
\hline $\mathrm{C}$ & -2.19026900 & -2.71957700 & 1.65561400 \\
\hline $\mathrm{C}$ & -2.81757900 & -2.80027700 & 0.38684000 \\
\hline $\mathrm{C}$ & -3.58297500 & -1.60812400 & 0.17264800 \\
\hline $\mathrm{H}$ & -0.07562800 & 0.16856600 & 1.07206000 \\
\hline $\mathrm{H}$ & 1.27509400 & 1.37300000 & 0.52679400 \\
\hline $\mathrm{H}$ & 0.23848600 & -1.87817000 & 0.76573600 \\
\hline $\mathrm{H}$ & -1.78427500 & 0.72928600 & 0.16638500 \\
\hline $\mathrm{H}$ & 2.34762700 & 0.99220800 & -1.95942800 \\
\hline $\mathrm{H}$ & 0.33544000 & 0.77832200 & -3.33727800 \\
\hline $\mathrm{H}$ & -1.59761800 & -0.45452400 & -2.58966900 \\
\hline $\mathrm{H}$ & -0.97144800 & -2.73255200 & -1.91858200 \\
\hline $\mathrm{H}$ & 0.17970600 & -4.29369500 & -0.59428300 \\
\hline $\mathrm{H}$ & 1.51225800 & -3.87105400 & 0.49524000 \\
\hline $\mathrm{H}$ & -5.99364500 & -1.88678200 & -1.18140300 \\
\hline $\mathrm{H}$ & -4.91153100 & 0.26058000 & -1.34273100 \\
\hline $\mathrm{H}$ & -4.03948500 & -1.65327600 & -2.55699800 \\
\hline $\mathrm{H}$ & -3.86408300 & 0.16316600 & 1.51862500 \\
\hline $\mathrm{H}$ & -2.23768800 & -1.11297100 & 3.21578100 \\
\hline $\mathrm{H}$ & -1.53914700 & -3.46558500 & 2.09417900 \\
\hline $\mathrm{H}$ & -2.72766600 & -3.62683300 & -0.30633500 \\
\hline $\mathrm{H}$ & -2.88453900 & 2.88549600 & -0.30088900 \\
\hline $\mathrm{H}$ & -1.76384400 & 2.78726600 & -2.73155100 \\
\hline $\mathrm{H}$ & 0.84309000 & 3.43322400 & -2.46988900 \\
\hline $\mathrm{H}$ & 1.32080800 & 3.91970600 & 0.12052600 \\
\hline $\mathrm{H}$ & -2.18127000 & 2.85122600 & 2.77669600 \\
\hline $\mathrm{H}$ & 0.16126300 & 3.46045200 & 3.00449400 \\
\hline $\mathrm{H}$ & -1.49570600 & 5.14258600 & 2.55305300 \\
\hline $\mathrm{H}$ & 6.50343200 & -0.30645100 & -0.88707900 \\
\hline $\mathrm{H}$ & 4.76708500 & -1.21687800 & -2.28359100 \\
\hline $\mathrm{H}$ & 4.80436400 & 1.15398000 & -1.74190700 \\
\hline $\mathrm{H}$ & 3.65777800 & -2.76141200 & 0.15890400 \\
\hline $\mathrm{H}$ & 2.43050000 & -2.49655900 & 2.53653400 \\
\hline $\mathrm{H}$ & 2.52070000 & 0.10198800 & 3.23168500 \\
\hline $\mathrm{H}$ & 3.80872100 & 1.43292400 & 1.29595700 \\
\hline $\mathrm{H}$ & 1.75270800 & -3.83486400 & -1.25584700 \\
\hline
\end{tabular}




\begin{tabular}{|c|c|c|c|}
\hline $\mathrm{B8}^{\mathrm{C}}$ & & & \\
\hline $\mathrm{Ti}$ & -0.48982600 & 1.37560700 & -0.56065300 \\
\hline $\mathrm{Ti}$ & 1.55928700 & -0.05742800 & 0.45443700 \\
\hline $\mathrm{Ti}$ & -0.95237100 & -1.06067800 & 0.41120800 \\
\hline $\mathrm{Si}$ & -2.06720400 & 3.50881400 & 2.04153200 \\
\hline $\mathrm{Si}$ & 4.93737400 & -0.51506600 & -1.25804500 \\
\hline $\mathrm{Si}$ & -4.02089700 & -2.44800700 & -1.30527200 \\
\hline $\mathrm{C}$ & 1.44723500 & 0.58967900 & -1.55630400 \\
\hline $\mathrm{C}$ & 0.34378000 & 0.40016100 & -2.36665400 \\
\hline $\mathrm{C}$ & -0.67340400 & -0.56768900 & -1.90383900 \\
\hline $\mathrm{C}$ & -0.10237800 & -1.86357900 & -1.48359800 \\
\hline $\mathrm{C}$ & 0.96711500 & -1.88266400 & -0.61205100 \\
\hline $\mathrm{C}$ & 1.64054200 & -3.22053100 & -0.37408100 \\
\hline $\mathrm{C}$ & -1.61865100 & 3.30451100 & 0.24235000 \\
\hline $\mathrm{C}$ & -0.42247600 & 3.75141700 & -0.40559500 \\
\hline $\mathrm{C}$ & -0.50507600 & 3.45266900 & -1.78668300 \\
\hline $\mathrm{C}$ & -1.75720200 & 2.82558200 & -2.02331500 \\
\hline $\mathrm{C}$ & -2.43901700 & 2.74041300 & -0.78751400 \\
\hline $\mathrm{C}$ & 3.99121500 & -0.16960800 & 0.31152900 \\
\hline $\mathrm{C}$ & 3.62942100 & 1.11075600 & 0.82836400 \\
\hline $\mathrm{C}$ & 3.01056400 & 0.94648100 & 2.09500600 \\
\hline $\mathrm{C}$ & 2.97200700 & -0.43954100 & 2.37904000 \\
\hline $\mathrm{C}$ & 3.56157200 & -1.12294600 & 1.28464200 \\
\hline $\mathrm{C}$ & -3.16614100 & -1.34669800 & 1.29249800 \\
\hline $\mathrm{C}$ & -2.25916100 & -1.62999100 & 2.33579800 \\
\hline $\mathrm{C}$ & -1.52314500 & -2.79256500 & 1.96741600 \\
\hline $\mathrm{C}$ & -1.98000600 & -3.21400400 & 0.69783600 \\
\hline $\mathrm{C}$ & -2.99823000 & -2.31395300 & 0.24890200 \\
\hline $\mathrm{H}$ & -0.20979200 & 0.51139500 & 1.03377100 \\
\hline $\mathrm{H}$ & 0.99851300 & 1.73633000 & 0.37940500 \\
\hline $\mathrm{H}$ & 0.46068400 & -1.14996800 & 1.53804200 \\
\hline $\mathrm{H}$ & -1.87855000 & 0.43821400 & 0.09900000 \\
\hline $\mathrm{H}$ & 2.23309400 & 1.23895900 & -1.95249200 \\
\hline $\mathrm{H}$ & 0.23238500 & 0.83479600 & -3.36180400 \\
\hline $\mathrm{H}$ & -1.56396800 & -0.63871200 & -2.52799700 \\
\hline $\mathrm{H}$ & -0.48775100 & -2.76914800 & -1.96063800 \\
\hline $\mathrm{H}$ & 1.09233900 & -4.04673700 & -0.85051900 \\
\hline $\mathrm{H}$ & 1.74150600 & -3.45250400 & 0.69186700 \\
\hline $\mathrm{H}$ & -5.16854000 & -3.37879800 & -1.11934000 \\
\hline $\mathrm{H}$ & -4.56447500 & -1.11210800 & -1.66828300 \\
\hline $\mathrm{H}$ & -3.19749500 & -2.96724100 & -2.43051300 \\
\hline $\mathrm{H}$ & -3.86024700 & -0.51606400 & 1.27272000 \\
\hline $\mathrm{H}$ & -2.12686200 & -1.05192300 & 3.24207000 \\
\hline $\mathrm{H}$ & -0.74159600 & -3.26059700 & 2.55199300 \\
\hline $\mathrm{H}$ & -1.60393000 & -4.06765000 & 0.14771200 \\
\hline
\end{tabular}




$\begin{array}{lrrr}\mathrm{H} & -3.41775000 & 2.30081100 & -0.64061900 \\ \mathrm{H} & -2.11661700 & 2.45592900 & -2.97613300 \\ \mathrm{H} & 0.26410800 & 3.64082200 & -2.52532400 \\ \mathrm{H} & 0.41787000 & 4.22683500 & 0.08474600 \\ \mathrm{H} & -3.02080400 & 2.44487400 & 2.45397700 \\ \mathrm{H} & -0.84925600 & 3.44712600 & 2.89176900 \\ \mathrm{H} & -2.72487000 & 4.82405000 & 2.28785400 \\ \mathrm{H} & 6.40326700 & -0.57049700 & -0.98997500 \\ \mathrm{H} & 4.53973000 & -1.82393900 & -1.83956000 \\ \mathrm{H} & 4.70719100 & 0.56924900 & -2.24903400 \\ \mathrm{H} & 3.67896800 & -2.19553600 & 1.20778600 \\ \mathrm{H} & 2.54435500 & -0.89896200 & 3.26137300 \\ \mathrm{H} & 2.61016200 & 1.73886900 & 2.71529000 \\ \mathrm{H} & 3.77926500 & 2.05853200 & 0.32480000 \\ \mathrm{H} & 2.65187500 & -3.21924000 & -0.79925300\end{array}$

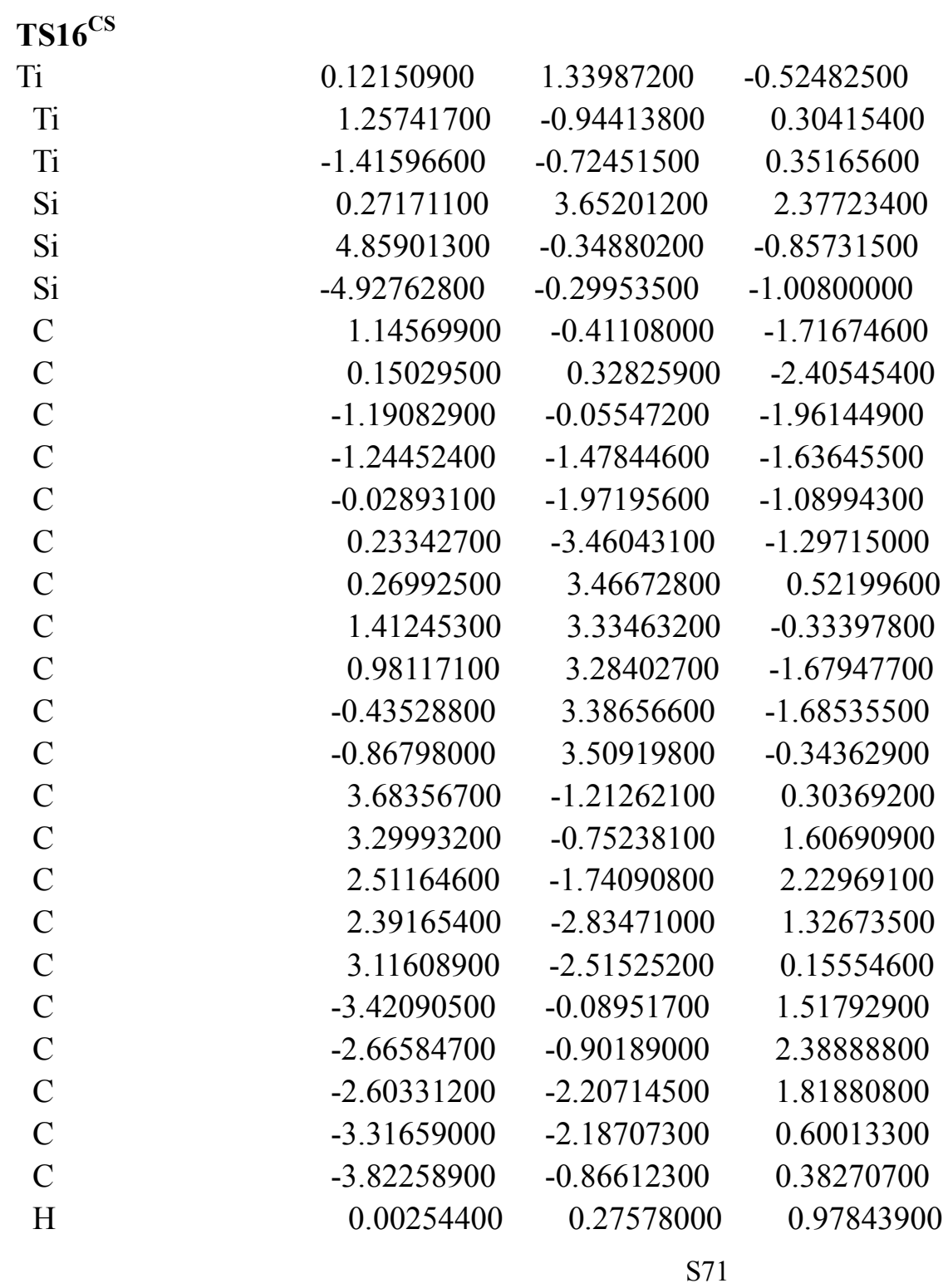




\begin{tabular}{|c|c|c|c|}
\hline $\mathrm{H}$ & 1.65301700 & 0.84063300 & 0.33000300 \\
\hline $\mathrm{H}$ & -0.12276000 & -1.55388800 & 1.34593200 \\
\hline $\mathrm{H}$ & -1.49802900 & 1.08920200 & 0.28001500 \\
\hline $\mathrm{H}$ & 2.12603600 & -0.47994500 & -2.19738400 \\
\hline $\mathrm{H}$ & 0.30984400 & 0.82303900 & -3.36151900 \\
\hline $\mathrm{H}$ & -2.05688600 & 0.41521600 & -2.42046300 \\
\hline $\mathrm{H}$ & -1.98245700 & -2.12852300 & -2.10416500 \\
\hline $\mathrm{H}$ & -0.68779000 & -3.97616600 & -1.60065200 \\
\hline $\mathrm{H}$ & 0.62905300 & -3.97131100 & -0.41652900 \\
\hline $\mathrm{H}$ & -6.35718000 & -0.60663600 & -0.72246800 \\
\hline $\mathrm{H}$ & -4.80315100 & 1.17139500 & -1.19093700 \\
\hline $\mathrm{H}$ & -4.56639000 & -0.98599700 & -2.27811800 \\
\hline $\mathrm{H}$ & -3.63747200 & 0.96067500 & 1.66827700 \\
\hline $\mathrm{H}$ & -2.19680800 & -0.58560200 & 3.31230500 \\
\hline $\mathrm{H}$ & -2.08611200 & -3.05941600 & 2.24125700 \\
\hline $\mathrm{H}$ & -3.44210200 & -3.02912800 & -0.06921500 \\
\hline $\mathrm{H}$ & -1.89856700 & 3.59559600 & -0.02237500 \\
\hline $\mathrm{H}$ & -1.07360400 & 3.35713100 & -2.56020600 \\
\hline $\mathrm{H}$ & 1.61547200 & 3.15782900 & -2.54791700 \\
\hline $\mathrm{H}$ & 2.44104000 & 3.26675600 & -0.00177700 \\
\hline $\mathrm{H}$ & -1.01014800 & 3.15642000 & 2.94502700 \\
\hline $\mathrm{H}$ & 1.40700300 & 2.89936200 & 2.97390400 \\
\hline $\mathrm{H}$ & 0.42020500 & 5.08065900 & 2.77819500 \\
\hline $\mathrm{H}$ & 6.26267000 & -0.79061300 & -0.62597400 \\
\hline $\mathrm{H}$ & 4.52366200 & -0.65716800 & -2.27448400 \\
\hline $\mathrm{H}$ & 4.80014200 & 1.12202600 & -0.64676200 \\
\hline $\mathrm{H}$ & 3.21015700 & -3.14683000 & -0.71975400 \\
\hline $\mathrm{H}$ & 1.83516900 & -3.74497500 & 1.51074500 \\
\hline $\mathrm{H}$ & 2.04987600 & -1.67006800 & 3.20688600 \\
\hline $\mathrm{H}$ & 3.55456400 & 0.21076200 & 2.03211600 \\
\hline $\mathrm{H}$ & 0.95615800 & -3.60093000 & -2.11134100 \\
\hline \multicolumn{4}{|c|}{$B 9^{\mathrm{CS}}$} \\
\hline $\mathrm{Ti}$ & 0.32868900 & 1.28375500 & -0.56236800 \\
\hline $\mathrm{Ti}$ & 1.11742000 & -1.09160300 & 0.31025800 \\
\hline $\mathrm{Ti}$ & -1.48083500 & -0.54922200 & 0.38757800 \\
\hline $\mathrm{Si}$ & 0.66727600 & 3.55251600 & 2.34538200 \\
\hline $\mathrm{Si}$ & 4.76711500 & -0.48326100 & -0.49374900 \\
\hline $\mathrm{Si}$ & -5.04679900 & 0.03472600 & -0.85363700 \\
\hline $\mathrm{C}$ & 0.78479300 & -0.74738400 & -1.85455900 \\
\hline $\mathrm{C}$ & 0.02858600 & 0.36163800 & -2.46976500 \\
\hline $\mathrm{C}$ & -1.35084000 & 0.12803700 & -2.19788500 \\
\hline $\mathrm{C}$ & -1.56534700 & -1.17487900 & -1.63809000 \\
\hline $\mathrm{C}$ & -0.23506900 & -1.77906100 & -1.36294300 \\
\hline $\mathrm{C}$ & -0.13992600 & -3.26664600 & -1.70548400 \\
\hline $\mathrm{C}$ & 0.77321700 & 3.35928600 & 0.49414200 \\
\hline
\end{tabular}




\begin{tabular}{|c|c|c|c|}
\hline $\mathrm{C}$ & 1.93589500 & 3.03085100 & -0.27238700 \\
\hline $\mathrm{C}$ & 1.60777200 & 3.05001700 & -1.64967100 \\
\hline $\mathrm{C}$ & 0.23824700 & 3.39518500 & -1.76388800 \\
\hline $\mathrm{C}$ & -0.27380000 & 3.58361300 & -0.45674000 \\
\hline $\mathrm{C}$ & 3.51022300 & -1.52576000 & 0.40792400 \\
\hline $\mathrm{C}$ & 3.02660700 & -1.32291900 & 1.74360300 \\
\hline $\mathrm{C}$ & 2.13539600 & -2.36650800 & 2.07234800 \\
\hline $\mathrm{C}$ & 2.04213300 & -3.23360500 & 0.94506900 \\
\hline $\mathrm{C}$ & 2.89459100 & -2.72216800 & -0.06113500 \\
\hline $\mathrm{C}$ & -3.38308000 & 0.31844800 & 1.56374800 \\
\hline $\mathrm{C}$ & -2.64990700 & -0.48381600 & 2.46519300 \\
\hline $\mathrm{C}$ & -2.72155200 & -1.82870900 & 2.00088500 \\
\hline $\mathrm{C}$ & -3.49458500 & -1.84388500 & 0.81755200 \\
\hline $\mathrm{C}$ & -3.91095300 & -0.51013500 & 0.52125100 \\
\hline $\mathrm{H}$ & 0.04444300 & 0.32867200 & 1.01341800 \\
\hline $\mathrm{H}$ & 1.80195100 & 0.56712300 & 0.19449900 \\
\hline $\mathrm{H}$ & -0.29705000 & -1.58703000 & 1.29660800 \\
\hline $\mathrm{H}$ & -1.35456800 & 1.26250100 & 0.15614400 \\
\hline $\mathrm{H}$ & 1.71776200 & -1.04397900 & -2.33696600 \\
\hline $\mathrm{H}$ & 0.37927500 & 0.95637600 & -3.30707200 \\
\hline $\mathrm{H}$ & -2.15175100 & 0.76868400 & -2.55455200 \\
\hline $\mathrm{H}$ & -2.43280300 & -1.77543700 & -1.90020300 \\
\hline $\mathrm{H}$ & -0.87655900 & -3.84522600 & -1.13511100 \\
\hline $\mathrm{H}$ & 0.84023900 & -3.69873700 & -1.49840800 \\
\hline $\mathrm{H}$ & -6.47492300 & -0.04591500 & -0.43853500 \\
\hline $\mathrm{H}$ & -4.76013100 & 1.44523200 & -1.23056800 \\
\hline $\mathrm{H}$ & -4.87422900 & -0.83669500 & -2.04767800 \\
\hline $\mathrm{H}$ & -3.50771900 & 1.39174800 & 1.63701500 \\
\hline $\mathrm{H}$ & -2.11257000 & -0.13658000 & 3.33872700 \\
\hline $\mathrm{H}$ & -2.25665700 & -2.68689500 & 2.46903700 \\
\hline $\mathrm{H}$ & -3.71605900 & -2.72144200 & 0.22134800 \\
\hline $\mathrm{H}$ & -1.29675500 & 3.84519800 & -0.21528100 \\
\hline $\mathrm{H}$ & -0.32634200 & 3.47656000 & -2.68471300 \\
\hline $\mathrm{H}$ & 2.27849400 & 2.81273900 & -2.46692000 \\
\hline $\mathrm{H}$ & 2.91025700 & 2.79388400 & 0.13579400 \\
\hline $\mathrm{H}$ & -0.70681900 & 3.24195200 & 2.82113200 \\
\hline $\mathrm{H}$ & 1.64499400 & 2.65494100 & 3.01607400 \\
\hline $\mathrm{H}$ & 0.98184400 & 4.94972000 & 2.76222000 \\
\hline $\mathrm{H}$ & 6.12822500 & -1.08291200 & -0.40100500 \\
\hline $\mathrm{H}$ & 4.42443800 & -0.37704400 & -1.93836500 \\
\hline $\mathrm{H}$ & 4.82643300 & 0.87573300 & 0.10513800 \\
\hline $\mathrm{H}$ & 3.03597500 & -3.15221500 & -1.04608200 \\
\hline $\mathrm{H}$ & 1.42408100 & -4.11957500 & 0.87393500 \\
\hline $\mathrm{H}$ & 1.59653700 & -2.47715700 & 3.00489000 \\
\hline $\mathrm{H}$ & 3.28288900 & -0.48564200 & 2.38217900 \\
\hline $\mathrm{H}$ & -0.34961800 & -3.42838900 & -2.77394100 \\
\hline
\end{tabular}




\begin{tabular}{|c|c|c|c|}
\hline $2^{\mathrm{CS}}$ & & & \\
\hline $\mathrm{Ti}$ & 1.49449400 & -0.59071400 & 0.41894400 \\
\hline $\mathrm{Ti}$ & -1.24829300 & -0.88855400 & 0.34539600 \\
\hline $\mathrm{Ti}$ & -0.08273300 & 1.34833200 & -0.41485600 \\
\hline $\mathrm{Si}$ & 5.00405700 & 0.06225000 & -0.86240900 \\
\hline $\mathrm{Si}$ & -4.76607800 & -1.00577500 & -1.08186500 \\
\hline $\mathrm{Si}$ & -1.27741800 & 4.05272200 & 1.87193300 \\
\hline $\mathrm{C}$ & 0.32460300 & -2.29628200 & -1.38920200 \\
\hline $\mathrm{C}$ & 1.41917900 & -1.39194600 & -1.57109600 \\
\hline $\mathrm{C}$ & 0.86577400 & -0.04441600 & -1.85128500 \\
\hline $\mathrm{C}$ & -0.64730200 & -0.20653900 & -1.89241900 \\
\hline $\mathrm{C}$ & -0.91654200 & -1.64125800 & -1.64603100 \\
\hline $\mathrm{C}$ & 0.47871200 & -3.78478900 & -1.22030900 \\
\hline $\mathrm{H}$ & 1.27160600 & -4.03766000 & -0.51030100 \\
\hline $\mathrm{H}$ & 0.74204900 & -4.24510000 & -2.18300900 \\
\hline $\mathrm{H}$ & -0.44641700 & -4.25179700 & -0.87090100 \\
\hline $\mathrm{C}$ & 3.92469400 & -0.59656800 & 0.50734900 \\
\hline $\mathrm{C}$ & 3.48369200 & -1.94083500 & 0.69903500 \\
\hline $\mathrm{C}$ & 2.73163000 & -2.01123400 & 1.89682700 \\
\hline $\mathrm{C}$ & 2.70472000 & -0.71192800 & 2.47739400 \\
\hline $\mathrm{C}$ & 3.43587000 & 0.14833400 & 1.62954900 \\
\hline $\mathrm{C}$ & -3.62156400 & -1.41509700 & 0.33069100 \\
\hline $\mathrm{C}$ & -3.34977800 & -0.58580900 & 1.46740200 \\
\hline $\mathrm{C}$ & -2.48957400 & -1.27372700 & 2.35070200 \\
\hline $\mathrm{C}$ & -2.21515800 & -2.54756300 & 1.77794600 \\
\hline $\mathrm{C}$ & -2.91394700 & -2.63534600 & 0.54964400 \\
\hline $\mathrm{C}$ & -0.66231700 & 3.60370100 & 0.16809100 \\
\hline $\mathrm{C}$ & -1.46672800 & 3.24651200 & -0.96363300 \\
\hline $\mathrm{C}$ & -0.62484900 & 3.04036000 & -2.07895900 \\
\hline $\mathrm{C}$ & 0.71594000 & 3.25500800 & -1.66231200 \\
\hline $\mathrm{C}$ & 0.68994400 & 3.61171700 & -0.28949200 \\
\hline $\mathrm{H}$ & -0.01076700 & 0.24637900 & 1.16842700 \\
\hline $\mathrm{H}$ & 0.20326500 & -1.66480200 & 1.15735200 \\
\hline $\mathrm{H}$ & -1.67172300 & 0.86920100 & 0.28918400 \\
\hline $\mathrm{H}$ & 1.52515400 & 1.21821700 & 0.38374800 \\
\hline $\mathrm{H}$ & 1.40230700 & 0.56488000 & -2.57834900 \\
\hline $\mathrm{H}$ & -1.26608600 & 0.28706200 & -2.64102300 \\
\hline $\mathrm{H}$ & -1.77519700 & -2.17074300 & -2.04845500 \\
\hline $\mathrm{H}$ & 2.38177500 & -1.72941900 & -1.94562300 \\
\hline $\mathrm{H}$ & 4.86147800 & 1.54041100 & -0.94508900 \\
\hline $\mathrm{H}$ & 6.44139800 & -0.25106400 & -0.62752800 \\
\hline $\mathrm{H}$ & 4.62776800 & -0.54028800 & -2.17136700 \\
\hline $\mathrm{H}$ & 3.68025100 & -2.77162900 & 0.03198600 \\
\hline $\mathrm{H}$ & 2.25516100 & -2.89598400 & 2.29999100 \\
\hline $\mathrm{H}$ & 2.19502000 & -0.42965000 & 3.38992100 \\
\hline
\end{tabular}




$\begin{array}{lrrr}\mathrm{H} & 3.58780900 & 1.20811600 & 1.79102500 \\ \mathrm{H} & -4.28040800 & -1.62874800 & -2.34423800 \\ \mathrm{H} & -6.14463200 & -1.51443300 & -0.83615200 \\ \mathrm{H} & -4.84706300 & 0.46846600 & -1.26121500 \\ \mathrm{H} & -3.72930500 & 0.41719100 & 1.61659700 \\ \mathrm{H} & -2.08981900 & -0.89179200 & 3.28155200 \\ \mathrm{H} & -1.57719800 & -3.31109600 & 2.20473700 \\ \mathrm{H} & -2.90220700 & -3.48704600 & -0.11975000 \\ \mathrm{H} & -1.46899500 & 5.52465500 & 2.00566800 \\ \mathrm{H} & -2.58745900 & 3.39823700 & 2.12905300 \\ \mathrm{H} & -0.29778900 & 3.62921300 & 2.90822500 \\ \mathrm{H} & -2.54386700 & 3.13155700 & -0.95760200 \\ \mathrm{H} & -0.94384400 & 2.74226000 & -3.07042100 \\ \mathrm{H} & 1.60173900 & 3.15992600 & -2.27800300 \\ \mathrm{H} & 1.55985000 & 3.83355800 & 0.31631900\end{array}$

$1^{T}$

Ti

Ti

Ti

$\mathrm{Si}$

$\mathrm{Si}$

$\mathrm{Si}$

C

C

C

C

C

C

C

C

C

C

C

C

C

C

C

$\mathrm{H}$

$\mathrm{H}$

$\mathrm{H}$

$\mathrm{H}$

$\mathrm{H}$

$\mathrm{H}$

$\mathrm{H}$

$\begin{array}{ccc}0.43566100 & 1.34192000 & -0.38045700 \\ -1.47029600 & -0.61278500 & -0.51279400 \\ 0.96718200 & -1.15921100 & 0.61080800 \\ -4.48979100 & -0.64866000 & 1.61904900 \\ 0.27460800 & 4.22639900 & 1.93769700 \\ 4.14366200 & -1.74939700 & -1.24033500 \\ -3.74561500 & -0.98659600 & -0.05762500 \\ -3.18056900 & -2.22051700 & -0.52257400 \\ -2.78379300 & -2.07332300 & -1.87605300 \\ -3.08793500 & -0.74829000 & -2.27558600 \\ -3.67284600 & -0.08345300 & -1.16698000 \\ 0.72919200 & 3.58702100 & 0.24526700 \\ 2.00243900 & 3.07490000 & -0.17331100 \\ 1.95150900 & 2.76622400 & -1.55645200 \\ 0.64859600 & 3.07357800 & -2.02151600 \\ -0.09782300 & 3.57340700 & -0.92353100 \\ 3.02390900 & -2.22580800 & 0.18356900 \\ 1.99612300 & -3.22476600 & 0.17150900 \\ 1.42615200 & -3.31895000 & 1.46677300 \\ 2.09171200 & -2.37931600 & 2.29878500 \\ 3.07042700 & -1.71411200 & 1.51943900 \\ 1.45987500 & 0.50834600 & 1.12547700 \\ 1.54134600 & -0.16459900 & -0.74994700 \\ -1.43407000 & 1.23170900 & -0.10494300 \\ -0.50157600 & 0.43187200 & -1.70156700 \\ -0.71919300 & -1.70095000 & 0.98638800 \\ 0.07629000 & -1.67055000 & -0.84493100 \\ -0.32093300 & 0.09419300 & 0.75943800\end{array}$

S75 


$\begin{array}{lrrr}\mathrm{H} & -5.94234900 & -0.98490600 & 1.64688700 \\ \mathrm{H} & -3.81575600 & -1.47957900 & 2.65238800 \\ \mathrm{H} & -4.34805400 & 0.79063700 & 1.96146000 \\ \mathrm{H} & -3.05261900 & -3.11609400 & 0.07412600 \\ \mathrm{H} & -2.29634900 & -2.82597500 & -2.48368100 \\ \mathrm{H} & -2.87462900 & -0.30869800 & -3.24166300 \\ \mathrm{H} & -3.98787200 & 0.95280100 & -1.15373300 \\ \mathrm{H} & 1.69007300 & -3.80182200 & -0.69180800 \\ \mathrm{H} & 0.61329500 & -3.96940600 & 1.76124600 \\ \mathrm{H} & 1.85891600 & -2.17288500 & 3.33783800 \\ \mathrm{H} & 3.72624100 & -0.92752200 & 1.87041500 \\ \mathrm{H} & 4.62948600 & -0.35824000 & -1.05677700 \\ \mathrm{H} & 5.32946000 & -2.65152300 & -1.27691900 \\ \mathrm{H} & 3.42269700 & -1.88332300 & -2.53052800 \\ \mathrm{H} & 2.75197000 & 2.33202200 & -2.14289700 \\ \mathrm{H} & 0.27617700 & 2.91569400 & -3.02574000 \\ \mathrm{H} & -1.13905600 & 3.87006500 & -0.95824000 \\ \mathrm{H} & 2.86016800 & 2.92206800 & 0.47118000 \\ \mathrm{H} & -1.18743500 & 4.08274500 & 2.16322500 \\ \mathrm{H} & 1.02026000 & 3.47893300 & 2.98533200 \\ \mathrm{H} & 0.62002500 & 5.66988600 & 2.08168500\end{array}$

A1 $^{\text {T }}$

$\mathrm{Ti}$

Ti

$\mathrm{Si}$

$\mathrm{Si}$

$\mathrm{Si}$

C

C

C

C

C

C

C

C

C

C

C

C

C

C

C

$\mathrm{H}$

$\mathrm{H}$

$$
\begin{aligned}
& 1.02495200 \\
& 0.03960200
\end{aligned}
$$

$-1.67505800$

$-0.65076200$

0.20580500

1.08424700

$-4.84423000$

0.51269500

0.32617800

4.42671200

$-0.19971300$

$-1.17079400$

1.97291200

$-0.46969800$

4.19998200

$-4.02232700$

$-1.07028700$

$-0.23979900$

$-3.60724000$

$-2.16768600$

0.58069600

$-3.07887100$

$-3.19149400$

$-0.24841000$

$-3.16032400$

$-2.74795000$

$-1.59542200$

$-3.73199100$

$-1.45230000$

$-1.58773700$

3.32397900

$-1.70305100$

$-1.28777800$

3.14004600

$-2.71544100$

$-0.29051400$

2.26500100

$-3.70542500$

$-0.79428300$

1.88648700

$-3.31927500$

$-2.10930400$

2.53920700

$-2.09476200$

$-2.41512900$

1.00319800

0.76339900

3.18716700

$-0.39748700$

1.02421700

3.27418300

$-0.73259100$

2.05383900

2.35771500

0.45379200

2.45448700

1.68520000

1.51485500

1.66133300

2.19338000

$-0.58990800$

$-1.72193100$

$-1.82686900$

$-0.52561300$

$-2.56923200$

$-0.02071400$

$\mathrm{H}$

$-1.29131700$

$-1.13885800$

1.28342200 


$\begin{array}{lrrr}\mathrm{H} & 1.01972400 & -1.36949100 & 1.17939900 \\ \mathrm{H} & 1.48348600 & 0.08399000 & -0.14811700 \\ \mathrm{H} & -1.72775500 & 2.44104600 & 2.17603900 \\ \mathrm{H} & -1.09760400 & 0.49511000 & 3.91037400 \\ \mathrm{H} & 0.52903300 & 3.20464400 & 0.90791900 \\ \mathrm{H} & 2.54466700 & 1.70422300 & 1.86000800 \\ \mathrm{H} & 3.17465100 & -0.92646700 & 3.45512800 \\ \mathrm{H} & 2.43988600 & 0.14655700 & 5.47572100 \\ \mathrm{H} & 1.11846600 & -1.63460400 & 4.55034700 \\ \mathrm{H} & -2.66628900 & -4.13327100 & 0.08834000 \\ \mathrm{H} & -3.66947300 & -2.20462400 & 1.66068800 \\ \mathrm{H} & -2.81341800 & -3.28705100 & -2.46715600 \\ \mathrm{H} & -3.88890400 & -0.83175400 & -2.46416100 \\ \mathrm{H} & -6.32354500 & 0.33742200 & 0.36916000 \\ \mathrm{H} & -4.38409600 & 0.87798900 & 1.68937400 \\ \mathrm{H} & -4.54208900 & 1.60778700 & -0.63156400 \\ \mathrm{H} & 3.89936700 & 0.89155200 & -2.03188000 \\ \mathrm{H} & 4.53542300 & 0.26944200 & 0.23424500 \\ \mathrm{H} & 5.80257400 & -0.52250800 & -1.64778600 \\ \mathrm{H} & 3.57168500 & -2.70463700 & 0.70293600 \\ \mathrm{H} & 1.91575300 & -4.57959400 & -0.25859200 \\ \mathrm{H} & 1.20019600 & -3.85218200 & -2.75617900 \\ \mathrm{H} & -1.68171300 & -1.28992100 & -0.44162700 \\ \mathrm{C} & -0.20350000 & -0.28207800 & -0.69419900 \\ \mathrm{C} & -1.71788900 & 0.42479700 & 0.13809600 \\ \mathrm{C} & & & \\ \mathrm{C} & 2.44673000 & -1.54376000 & -3.34375100 \\ \mathrm{C} & 1.38753200 & 4.95953600 & -1.54957600 \\ \mathrm{C} & 0.18564800 & 5.59340900 & -1.22220100 \\ \mathrm{H} & -1.03092100 & 4.94351100 & -1.44687400 \\ \mathrm{H} & -1.04539500 & 3.65917900 & -1.99715000 \\ \mathrm{H} & 0.15658600 & 3.02389800 & -2.32145000 \\ \mathrm{H} & 1.37319500 & 3.67492800 & -2.09934000 \\ \mathrm{H} & 2.33342600 & 5.46715600 & -1.37875300 \\ \mathrm{H} & 0.19707800 & 6.59354400 & -0.79666600 \\ & -1.96582600 & 5.43826300 & -1.19628200 \\ \mathrm{H} & -1.99162500 & 3.15253300 & -2.16938700 \\ \mathrm{H} & 0.14755000 & 2.01919800 & -2.73555000 \\ \mathrm{H} & 2.30685400 & 3.17824400 & -2.34983100 \\ \mathrm{H} & & & \\ \mathrm{H} & & \end{array}$

\section{TS1 $^{\mathrm{T}}$}

Ti

Ti

$\mathrm{Si}$

$\mathrm{Si}$

Si

C

$\begin{array}{ccc}0.35075600 & -1.73760800 & -0.67909400 \\ 0.63841300 & 0.42936000 & 0.96495500 \\ -5.03704500 & 1.28177700 & -0.84389400 \\ 3.66377600 & -1.36635900 & -2.40656200 \\ 2.85378600 & -1.30325300 & 3.45858800 \\ -4.37940500 & -0.19615800 & 0.09575200\end{array}$




\begin{tabular}{|c|c|c|c|}
\hline $\mathrm{C}$ & -4.03227000 & -0.24279500 & 1.48337700 \\
\hline $\mathrm{C}$ & -3.62370600 & -1.55571500 & 1.81652300 \\
\hline $\mathrm{C}$ & -3.70661900 & -2.34922600 & 0.64488800 \\
\hline $\mathrm{C}$ & -4.16594200 & -1.51978700 & -0.40867300 \\
\hline $\mathrm{C}$ & 2.23678400 & -2.48363000 & -1.95455000 \\
\hline $\mathrm{C}$ & 2.13259400 & -3.31917800 & -0.79680700 \\
\hline $\mathrm{C}$ & 0.91681200 & -4.04070000 & -0.85528600 \\
\hline $\mathrm{C}$ & 0.24500200 & -3.65612200 & -2.04811300 \\
\hline $\mathrm{C}$ & 1.05499900 & -2.70440300 & -2.72469800 \\
\hline $\mathrm{C}$ & 2.25342800 & 0.30863000 & 2.72644300 \\
\hline $\mathrm{C}$ & 1.14921700 & 1.08752200 & 3.18308300 \\
\hline $\mathrm{C}$ & 1.04013100 & 2.25022700 & 2.37696100 \\
\hline $\mathrm{C}$ & 2.08491300 & 2.21300800 & 1.40900200 \\
\hline $\mathrm{C}$ & 2.82337300 & 1.02351200 & 1.62331300 \\
\hline $\mathrm{H}$ & -1.48999900 & -1.48534300 & -1.29759000 \\
\hline $\mathrm{H}$ & -0.96724500 & -2.04177200 & 0.59300700 \\
\hline $\mathrm{H}$ & -1.17609700 & 0.20706500 & 1.56308400 \\
\hline $\mathrm{H}$ & 0.98788800 & -1.36096400 & 1.02764500 \\
\hline $\mathrm{H}$ & 1.39783200 & -0.15338500 & -0.65254700 \\
\hline $\mathrm{H}$ & 0.28013000 & 3.01763300 & 2.46371100 \\
\hline $\mathrm{H}$ & 0.47460100 & 0.81344100 & 3.98548600 \\
\hline $\mathrm{H}$ & 2.25682100 & 2.94286500 & 0.62751800 \\
\hline $\mathrm{H}$ & 3.65746800 & 0.68508000 & 1.02078800 \\
\hline $\mathrm{H}$ & 3.55893400 & -2.11191000 & 2.43149200 \\
\hline $\mathrm{H}$ & 3.81525400 & -1.04029500 & 4.56782900 \\
\hline $\mathrm{H}$ & 1.71300800 & -2.07105700 & 4.02117000 \\
\hline $\mathrm{H}$ & -3.25447400 & -1.88226200 & 2.78123900 \\
\hline $\mathrm{H}$ & -4.03750700 & 0.60322200 & 2.16016300 \\
\hline $\mathrm{H}$ & -3.42725900 & -3.39134700 & 0.56072000 \\
\hline $\mathrm{H}$ & -4.31173600 & -1.83426400 & -1.43496400 \\
\hline $\mathrm{H}$ & -6.52881900 & 1.26734200 & -0.84825300 \\
\hline $\mathrm{H}$ & -4.59515800 & 2.53585200 & -0.18219900 \\
\hline $\mathrm{H}$ & -4.57940000 & 1.24676900 & -2.25568700 \\
\hline $\mathrm{H}$ & 3.18673400 & -0.21888200 & -3.22205800 \\
\hline $\mathrm{H}$ & 4.35106700 & -0.87006300 & -1.18587700 \\
\hline $\mathrm{H}$ & 4.67119600 & -2.10803800 & -3.21876200 \\
\hline $\mathrm{H}$ & 2.85124800 & -3.36329000 & 0.01261400 \\
\hline $\mathrm{H}$ & 0.55047200 & -4.73434600 & -0.10858500 \\
\hline $\mathrm{H}$ & -0.72632000 & -4.00798500 & -2.37343300 \\
\hline $\mathrm{H}$ & 0.80923900 & -2.21838300 & -3.66159200 \\
\hline $\mathrm{C}$ & 1.97141700 & 4.57320500 & -1.88356700 \\
\hline $\mathrm{C}$ & 1.25789900 & 5.36768400 & -0.98033800 \\
\hline $\mathrm{C}$ & 0.00314700 & 4.95424600 & -0.52551700 \\
\hline $\mathrm{C}$ & -0.53823900 & 3.74430300 & -0.97117000 \\
\hline $\mathrm{C}$ & 0.17544900 & 2.94948400 & -1.87448500 \\
\hline $\mathrm{C}$ & 1.43040700 & 3.36542800 & -2.33122700 \\
\hline
\end{tabular}




$\begin{array}{cccc}\mathrm{H} & 2.94529600 & 4.89836200 & -2.24063500 \\ \mathrm{H} & 1.67759000 & 6.30971200 & -0.63680100 \\ \mathrm{H} & -0.55524100 & 5.57721200 & 0.16888300 \\ \mathrm{H} & -1.51318200 & 3.41554400 & -0.62129100 \\ \mathrm{H} & -0.24831700 & 2.00972700 & -2.21669600 \\ \mathrm{H} & 1.98329600 & 2.74712000 & -3.03352900 \\ \mathrm{Ti} & -2.07342500 & -0.66317300 & 0.22267500 \\ \mathrm{H} & -0.50341100 & -0.09170100 & -0.42798900 \\ \mathrm{H} & -2.10388400 & 0.89208400 & -0.49797800\end{array}$

$\mathrm{A2}^{\mathrm{T}}$

$\mathrm{Ti}$

Ti

$\mathrm{Si}$

$\mathrm{Si}$

$\mathrm{Si}$

C

C

C

C

C

C

C

C

C

C

C

C

C

C

C

$\mathrm{H}$

$\mathrm{H}$

$\mathrm{H}$

$\mathrm{H}$

$\mathrm{H}$

$\mathrm{H}$

$\mathrm{H}$

$\mathrm{H}$

$\mathrm{H}$

$\mathrm{H}$

$\mathrm{H}$

H

$\mathrm{H}$

$\mathrm{H}$

$\mathrm{H}$

$\begin{array}{ccc}1.67444400 & -1.11373500 & -0.48049400 \\ 0.09951200 & 0.89943600 & 0.59388200 \\ -4.65701300 & -1.22515600 & 0.03653000 \\ 4.78584600 & 0.91526100 & -0.21691800 \\ -0.38704400 & -0.07805200 & 4.11808800 \\ -3.25061000 & -2.41494100 & -0.28484700 \\ -2.44442500 & -3.05732800 & 0.70549700 \\ -1.51157300 & -3.90254000 & 0.06186400 \\ -1.72077800 & -3.79464300 & -1.33986300 \\ -2.79211600 & -2.89082000 & -1.55468800 \\ 4.01195900 & -0.75046000 & -0.55010100 \\ 3.78016600 & -1.79701700 & 0.39899500 \\ 3.24528700 & -2.91649800 & -0.27819800 \\ 3.14407200 & -2.58563800 & -1.65778700 \\ 3.61428700 & -1.26137300 & -1.82996200 \\ -0.14729200 & 1.30943700 & 2.89179600 \\ -1.15985400 & 2.07371200 & 2.22811800 \\ -0.54121400 & 3.05195300 & 1.41577500 \\ 0.86521900 & 2.92237100 & 1.56274300 \\ 1.10813500 & 1.85935000 & 2.46802200 \\ 0.39377800 & -1.94881600 & -1.67283000 \\ 0.43789300 & -2.24949100 & 0.27766800 \\ -1.45991800 & -0.20323400 & 0.50001700 \\ 1.11608300 & -0.48558000 & 1.17381300 \\ 1.66506900 & 0.79674400 & -0.42987500 \\ -1.04852000 & 3.74158700 & 0.75243500 \\ -2.22731400 & 1.90232900 & 2.30045200 \\ 1.61659200 & 3.50784100 & 1.04820800 \\ 2.08386700 & 1.49427500 & 2.76205400 \\ 0.86740200 & -0.86410400 & 4.23647200 \\ -0.72335500 & 0.46305000 & 5.46624500 \\ -1.50348400 & -0.96899200 & 3.70559200 \\ -0.75199700 & -4.49955700 & 0.54833500 \\ -2.50265000 & -2.88093400 & 1.77331900 \\ -1.14746800 & -4.29470900 & -2.10966100\end{array}$




$\begin{array}{lrrc}\mathrm{H} & -3.19436700 & -2.61190300 & -2.51882800 \\ \mathrm{H} & -5.96764700 & -1.90661200 & -0.15534800 \\ \mathrm{H} & -4.58267100 & -0.73754600 & 1.43763000 \\ \mathrm{H} & -4.60905700 & -0.07070300 & -0.89947600 \\ \mathrm{H} & 4.38518900 & 1.88990500 & -1.26487700 \\ \mathrm{H} & 4.39319700 & 1.42622700 & 1.12334200 \\ \mathrm{H} & 6.27404000 & 0.81686700 & -0.23559000 \\ \mathrm{H} & 3.95370300 & -1.73147500 & 1.46660400 \\ \mathrm{H} & 2.93564200 & -3.84861600 & 0.17795800 \\ \mathrm{H} & 2.74247400 & -3.22360600 & -2.43517900 \\ \mathrm{H} & 3.65845000 & -0.72157500 & -2.76832000 \\ \mathrm{C} & 0.05556200 & 3.66553500 & -2.19764400 \\ \mathrm{C} & -0.68407200 & 4.81744900 & -1.92636300 \\ \mathrm{C} & -2.05650800 & 4.72277600 & -1.66609900 \\ \mathrm{C} & -2.68733500 & 3.47693100 & -1.67330600 \\ \mathrm{C} & -1.94858400 & 2.32042900 & -1.94843000 \\ \mathrm{C} & -0.57632700 & 2.41452100 & -2.21396900 \\ \mathrm{H} & 1.11958400 & 3.73535600 & -2.40806200 \\ \mathrm{H} & -0.19737400 & 5.78929200 & -1.92419500 \\ \mathrm{H} & -2.63238100 & 5.62215300 & -1.46335800 \\ \mathrm{H} & -3.75382800 & 3.40523800 & -1.47666500 \\ \mathrm{H} & -2.43291700 & 1.34879000 & -1.97749300 \\ \mathrm{H} & -0.01419600 & 1.52079500 & -2.46908700 \\ \mathrm{Ti} & -1.03607000 & -1.65748400 & -0.58535600 \\ \mathrm{H} & 0.07042300 & -0.24310700 & -0.88413400 \\ \mathrm{H} & -1.40678300 & -0.93865500 & -2.07099300\end{array}$

$\begin{array}{cccc}\text { TS2 }^{\mathbf{T}} & & & \\ \mathrm{Ti} & 1.71683700 & -0.98954000 & -0.51761600 \\ \mathrm{Ti} & -0.05074200 & 0.92731200 & 0.53761700 \\ \mathrm{Si} & -4.52921300 & -1.43550400 & 0.02310400 \\ \mathrm{Si} & 4.71005300 & 1.18761500 & -0.07330600 \\ \mathrm{Si} & -0.19420300 & -0.29475600 & 4.04902400 \\ \mathrm{C} & -3.06590700 & -2.55614800 & -0.28559100 \\ \mathrm{C} & -2.21831200 & -3.13970800 & 0.70892500 \\ \mathrm{C} & -1.25405700 & -3.95200000 & 0.07110400 \\ \mathrm{C} & -1.47918300 & -3.87753400 & -1.33062400 \\ \mathrm{C} & -2.59722100 & -3.03356100 & -1.55033900 \\ \mathrm{C} & 4.02898200 & -0.48919100 & -0.53009900 \\ \mathrm{C} & 3.84648700 & -1.60983500 & 0.34268400 \\ \mathrm{C} & 3.38919200 & -2.70935300 & -0.41959700 \\ \mathrm{C} & 3.28831800 & -2.29171300 & -1.77524300 \\ \mathrm{C} & 3.67998900 & -0.93417000 & -1.84861700 \\ \mathrm{C} & -0.23816300 & 1.16599300 & 2.88651400 \\ \mathrm{C} & -1.39068900 & 1.79041200 & 2.31657800 \\ \mathrm{C} & -0.97839200 & 2.88756400 & 1.52606900\end{array}$




\begin{tabular}{|c|c|c|c|}
\hline $\mathrm{C}$ & 0.43984700 & 2.97209000 & 1.59638800 \\
\hline $\mathrm{C}$ & 0.89273200 & 1.91937700 & 2.43088700 \\
\hline $\mathrm{H}$ & 0.51533800 & -1.83015300 & -1.76936500 \\
\hline $\mathrm{H}$ & 0.63619100 & -2.32731700 & 0.10887500 \\
\hline $\mathrm{H}$ & -1.54427800 & -0.21397800 & 0.30692300 \\
\hline $\mathrm{H}$ & 0.79115700 & -0.58023400 & 1.08254000 \\
\hline $\mathrm{H}$ & 1.63038400 & 0.88737100 & -0.27237600 \\
\hline $\mathrm{H}$ & -1.62343300 & 3.52157100 & 0.93039100 \\
\hline $\mathrm{H}$ & -2.41272900 & 1.44955900 & 2.43115900 \\
\hline $\mathrm{H}$ & 1.06227300 & 3.69481900 & 1.08459700 \\
\hline $\mathrm{H}$ & 1.92784300 & 1.69587700 & 2.65663000 \\
\hline $\mathrm{H}$ & 1.17398400 & -0.87131200 & 4.07950900 \\
\hline $\mathrm{H}$ & -0.54972300 & 0.12707400 & 5.43431400 \\
\hline $\mathrm{H}$ & -1.17170600 & -1.33692100 & 3.63813400 \\
\hline $\mathrm{H}$ & -0.46429200 & -4.50596400 & 0.56028300 \\
\hline $\mathrm{H}$ & -2.27626000 & -2.94925900 & 1.77433100 \\
\hline $\mathrm{H}$ & -0.88677300 & -4.35968800 & -2.09755500 \\
\hline $\mathrm{H}$ & -3.02416500 & -2.79601100 & -2.51483200 \\
\hline $\mathrm{H}$ & -5.80364100 & -2.19911500 & -0.09505800 \\
\hline $\mathrm{H}$ & -4.45045200 & -0.87758300 & 1.39792200 \\
\hline $\mathrm{H}$ & -4.57752500 & -0.32767600 & -0.96721000 \\
\hline $\mathrm{H}$ & 4.24230300 & 2.21878200 & -1.03599200 \\
\hline $\mathrm{H}$ & 4.30413400 & 1.56677000 & 1.30660000 \\
\hline $\mathrm{H}$ & 6.20094400 & 1.17757600 & -0.11033900 \\
\hline $\mathrm{H}$ & 4.00540300 & -1.60872100 & 1.41477600 \\
\hline $\mathrm{H}$ & 3.13055600 & -3.68759200 & -0.03412200 \\
\hline $\mathrm{H}$ & 2.93527000 & -2.89704300 & -2.60063600 \\
\hline $\mathrm{H}$ & 3.70431700 & -0.32997400 & -2.74773000 \\
\hline $\mathrm{C}$ & -0.10758400 & 3.89872000 & -1.96614700 \\
\hline $\mathrm{C}$ & -1.08304200 & 4.86234400 & -1.71126200 \\
\hline $\mathrm{C}$ & -2.42920900 & 4.48738400 & -1.61243400 \\
\hline $\mathrm{C}$ & -2.79706100 & 3.15007000 & -1.76464600 \\
\hline $\mathrm{C}$ & -1.82091500 & 2.17930000 & -2.02445200 \\
\hline $\mathrm{C}$ & -0.47443400 & 2.55443100 & -2.12659500 \\
\hline $\mathrm{H}$ & 0.93656100 & 4.18769100 & -2.05443900 \\
\hline $\mathrm{H}$ & -0.80118300 & 5.90591700 & -1.59795700 \\
\hline $\mathrm{H}$ & -3.18842800 & 5.24196300 & -1.42310500 \\
\hline $\mathrm{H}$ & -3.84195600 & 2.85973400 & -1.69424400 \\
\hline $\mathrm{H}$ & -2.10071400 & 1.14049700 & -2.17219600 \\
\hline $\mathrm{H}$ & 0.27724100 & 1.80992100 & -2.37331000 \\
\hline $\mathrm{Ti}$ & -0.89847500 & -1.69174800 & -0.61665000 \\
\hline $\mathrm{H}$ & 0.07893700 & -0.16029600 & -0.97522200 \\
\hline $\mathrm{H}$ & -1.32438400 & -1.08865900 & -2.12953700 \\
\hline
\end{tabular}

$\mathbf{A 3}^{\mathrm{T}}$

$\begin{array}{lll}-0.56611200 & -1.77082800 & -0.00930600\end{array}$ 


\begin{tabular}{|c|c|c|c|}
\hline $\mathrm{Ti}$ & 1.02128100 & 0.54094100 & 0.65693700 \\
\hline $\mathrm{Si}$ & -3.90719400 & 3.03173900 & -1.40631200 \\
\hline $\mathrm{Si}$ & 0.23394600 & -3.26214900 & -3.31244800 \\
\hline $\mathrm{Si}$ & 2.71995000 & -1.73685500 & 3.04348400 \\
\hline $\mathrm{C}$ & -3.64456100 & 2.01329900 & 0.13696900 \\
\hline $\mathrm{C}$ & -2.90289900 & 2.38519300 & 1.29919800 \\
\hline $\mathrm{C}$ & -2.98276600 & 1.33992600 & 2.25692100 \\
\hline $\mathrm{C}$ & -3.78444800 & 0.30948100 & 1.70740800 \\
\hline $\mathrm{C}$ & -4.17288200 & 0.70896200 & 0.40393400 \\
\hline $\mathrm{C}$ & -0.32038000 & -3.53976200 & -1.55483300 \\
\hline $\mathrm{C}$ & 0.50034500 & -3.82996900 & -0.41484800 \\
\hline $\mathrm{C}$ & -0.32821000 & -4.03902500 & 0.71767500 \\
\hline $\mathrm{C}$ & -1.67386200 & -3.88016300 & 0.30160500 \\
\hline $\mathrm{C}$ & -1.67057400 & -3.57437800 & -1.08266700 \\
\hline $\mathrm{C}$ & 2.46983500 & 0.00837800 & 2.42979100 \\
\hline $\mathrm{C}$ & 1.53998500 & 0.97449300 & 2.92888100 \\
\hline $\mathrm{C}$ & 1.73252200 & 2.19793700 & 2.24177300 \\
\hline $\mathrm{C}$ & 2.77645400 & 2.01177400 & 1.30234700 \\
\hline $\mathrm{C}$ & 3.23202600 & 0.67251500 & 1.41424800 \\
\hline $\mathrm{H}$ & -1.98052900 & -1.20628200 & 0.98448700 \\
\hline $\mathrm{H}$ & -0.39391300 & -0.31415600 & 1.45694800 \\
\hline $\mathrm{H}$ & -0.24254100 & 0.01594500 & -0.62074700 \\
\hline $\mathrm{H}$ & -0.42028200 & 1.70124300 & 0.54762400 \\
\hline $\mathrm{H}$ & 1.23032700 & -1.23942900 & 0.13900900 \\
\hline $\mathrm{H}$ & 1.15091200 & 3.10114400 & 2.37576700 \\
\hline $\mathrm{H}$ & 0.79106100 & 0.79169900 & 3.68938600 \\
\hline $\mathrm{H}$ & 3.13269500 & 2.74605000 & 0.59110600 \\
\hline $\mathrm{H}$ & 4.01672200 & 0.22265500 & 0.81850100 \\
\hline $\mathrm{H}$ & 3.37790500 & -2.55675400 & 1.99299800 \\
\hline $\mathrm{H}$ & 3.59778300 & -1.74707500 & 4.24823200 \\
\hline $\mathrm{H}$ & 1.42370100 & -2.35545800 & 3.42618200 \\
\hline $\mathrm{H}$ & -2.49595800 & 1.32167900 & 3.22432300 \\
\hline $\mathrm{H}$ & -2.34547800 & 3.30549600 & 1.42062000 \\
\hline $\mathrm{H}$ & -4.02668500 & -0.63118600 & 2.18332500 \\
\hline $\mathrm{H}$ & -4.75817500 & 0.11509200 & -0.28757700 \\
\hline $\mathrm{H}$ & -5.09459400 & 3.92331200 & -1.24268300 \\
\hline $\mathrm{H}$ & -2.73247600 & 3.90616500 & -1.65943200 \\
\hline $\mathrm{H}$ & -4.16341300 & 2.15076200 & -2.57309100 \\
\hline $\mathrm{H}$ & -0.78712300 & -2.47238100 & -4.04819600 \\
\hline $\mathrm{H}$ & 1.53417900 & -2.53705800 & -3.33584000 \\
\hline $\mathrm{H}$ & 0.43286600 & -4.55075900 & -4.03524500 \\
\hline $\mathrm{H}$ & 1.58300000 & -3.86685500 & -0.41290600 \\
\hline $\mathrm{H}$ & 0.00843800 & -4.25119900 & 1.72522300 \\
\hline $\mathrm{H}$ & -2.54899800 & -3.94546900 & 0.93560800 \\
\hline $\mathrm{H}$ & -2.54931600 & -3.36703000 & -1.68064200 \\
\hline $\mathrm{C}$ & 3.55191600 & 1.06032600 & -2.25643600 \\
\hline
\end{tabular}




$\begin{array}{lccc}\mathrm{C} & 4.12475300 & 2.33059600 & -2.22929800 \\ \mathrm{C} & 3.30838300 & 3.46611000 & -2.13139500 \\ \mathrm{C} & 1.92334700 & 3.32775200 & -2.05562400 \\ \mathrm{C} & 1.34034900 & 2.05207900 & -2.08706000 \\ \mathrm{C} & 2.15724300 & 0.91704000 & -2.18979300 \\ \mathrm{H} & 4.18101600 & 0.17867700 & -2.34799100 \\ \mathrm{H} & 5.20385800 & 2.44279600 & -2.29439800 \\ \mathrm{H} & 3.75769700 & 4.45588400 & -2.12166600 \\ \mathrm{H} & 1.28877300 & 4.20720200 & -1.98672400 \\ \mathrm{H} & 0.26003300 & 1.94391500 & -2.06651700 \\ \mathrm{H} & 1.71029300 & -0.06959500 & -2.27138500 \\ \mathrm{Ti} & -1.82236300 & 0.51763500 & 0.35107100 \\ \mathrm{H} & -1.83964900 & 1.14976800 & -1.21162000 \\ \mathrm{H} & -1.93713100 & -0.83294100 & -0.91301500\end{array}$

TS3 $^{\mathrm{T}}$

Ti

Ti

$\mathrm{Si}$

$\mathrm{Si}$

$\mathrm{Si}$

C

C

C

C

C

C

C

C

C

C

C

C

C

C

C

$\mathrm{H}$

$\mathrm{H}$

$\mathrm{H}$

$\mathrm{H}$

$\mathrm{H}$

H

$\mathrm{H}$

$\mathrm{H}$

$\mathrm{H}$

$\begin{array}{ccc}-1.31446300 & 1.43120100 & -0.08561000 \\ -0.04858400 & -0.98374400 & 0.66010500 \\ 4.96661800 & 0.74927700 & -1.27302800 \\ -3.48211100 & 1.25580900 & -3.09725900 \\ -2.41363400 & -0.80955300 & 3.51910500 \\ 3.83656600 & 1.69241500 & -0.13412400 \\ 3.47954900 & 1.34585800 & 1.20979200 \\ 2.63824700 & 2.35489800 & 1.73990900 \\ 2.45743300 & 3.34183300 & 0.74081800 \\ 3.18042700 & 2.94203100 & -0.41039800 \\ -3.15092000 & 2.03729800 & -1.43347300 \\ -3.65264000 & 1.61164900 & -0.15774600 \\ -3.23459700 & 2.53302000 & 0.83922800 \\ -2.46111000 & 3.53342700 & 0.20555700 \\ -2.40571500 & 3.23007600 & -1.17984600 \\ -0.96408700 & -1.66097200 & 2.70948100 \\ 0.42602100 & -1.40457500 & 2.93434800 \\ 1.19380200 & -2.34119200 & 2.19971800 \\ 0.29701100 & -3.18616000 & 1.50145700 \\ -1.02450100 & -2.77455200 & 1.80860500 \\ 0.12072200 & 2.49101100 & -0.17519400 \\ 0.01494100 & 0.82338300 & 1.21809400 \\ -0.01312300 & 0.26750800 & -0.81931000 \\ 1.65073400 & -0.53514200 & 0.27051900 \\ -1.75310400 & -0.33753900 & 0.24219800 \\ 2.27380900 & -2.37323000 & 2.14167300 \\ 0.82759700 & -0.60406400 & 3.54344300 \\ 0.57147200 & -3.97069600 & 0.80779400 \\ -1.93037600 & -3.21830800 & 1.41399800\end{array}$




$\begin{array}{lrrr}\mathrm{H} & -3.63213200 & -0.97567900 & 2.68385500 \\ \mathrm{H} & -2.69158200 & -1.39940200 & 4.85878300 \\ \mathrm{H} & -2.13592400 & 0.63842700 & 3.71133800 \\ \mathrm{H} & 2.17301700 & 2.34897300 & 2.71846600 \\ \mathrm{H} & 3.78491800 & 0.44512800 & 1.72797100 \\ \mathrm{H} & 1.83636000 & 4.22439200 & 0.82549100 \\ \mathrm{H} & 3.23474200 & 3.48944400 & -1.34184800 \\ \mathrm{H} & 6.39518000 & 1.13656300 & -1.06013200 \\ \mathrm{H} & 4.87646500 & -0.71324200 & -1.01386400 \\ \mathrm{H} & 4.64125000 & 1.03627000 & -2.69405200 \\ \mathrm{H} & -2.36967100 & 1.56665800 & -4.03139800 \\ \mathrm{H} & -3.61405000 & -0.21884100 & -2.94891100 \\ \mathrm{H} & -4.74933800 & 1.76684300 & -3.69019400 \\ \mathrm{H} & -4.25301600 & 0.72762400 & 0.01882900 \\ \mathrm{H} & -3.44142600 & 2.46204700 & 1.90031500 \\ \mathrm{H} & -1.96043600 & 4.36002600 & 0.69396800 \\ \mathrm{H} & -1.84883900 & 3.79331200 & -1.91965200 \\ \mathrm{C} & -1.30449700 & -3.74455700 & -1.81323200 \\ \mathrm{C} & -0.54232700 & -4.90966600 & -1.74923700 \\ \mathrm{C} & 0.85544300 & -4.83884200 & -1.83336600 \\ \mathrm{C} & 1.48662200 & -3.60403400 & -1.97563100 \\ \mathrm{C} & -2.72468800 & -2.42862400 & -2.04790600 \\ \mathrm{C} & -0.67300500 & -2.50048000 & -1.96724600 \\ \mathrm{H} & -2.38902300 & -3.79731300 & -1.76273000 \\ \mathrm{H} & -1.03086000 & -5.87480000 & -1.64370400 \\ \mathrm{H} & 1.44634600 & -5.75031700 & -1.79282600 \\ \mathrm{H} & 2.56945300 & -3.54674200 & -2.04426300 \\ \mathrm{H} & 1.21554200 & -1.47075300 & -2.19167600 \\ \mathrm{Ti} & -1.27089400 & -1.60018000 & -2.07542300 \\ \mathrm{H} & 1.54382900 & 1.29542900 & -0.09727100 \\ & 2.11049700 & 0.63208600 & -1.53976200 \\ \mathrm{H} & 1.39686800 & 1.72248400 & -1.73443300\end{array}$

\section{A4 ${ }^{\mathrm{T}}$}

$\mathrm{Ti}$

$\mathrm{Ti}$

$\mathrm{Si}$

$\mathrm{Si}$

$\mathrm{Si}$

C

C

C

C

C

C

C

$\begin{array}{ccc}1.81552300 & -0.72632000 & -0.06352400 \\ -0.48030600 & 0.68476700 & 0.62108900 \\ -4.23787700 & -2.29016700 & -1.66962600 \\ 4.14385500 & 0.45631600 & -2.71737300 \\ 0.95738700 & 0.44264100 & 4.01167400 \\ -2.75407100 & -2.90268500 & -0.72605800 \\ -2.48649500 & -2.68653500 & 0.66791700 \\ -1.31379900 & -3.39563400 & 1.02634100 \\ -0.83391600 & -4.06478400 & -0.12556300 \\ -1.70313500 & -3.76443800 & -1.20095700 \\ 3.93276900 & -0.41932200 & -1.08460800 \\ 3.98761800 & 0.16562900 & 0.22240600\end{array}$




\begin{tabular}{|c|c|c|c|}
\hline $\mathrm{C}$ & 3.85956000 & -0.85679200 & 1.19818800 \\
\hline $\mathrm{C}$ & 3.71113000 & -2.08931800 & 0.51798500 \\
\hline $\mathrm{C}$ & 3.75236600 & -1.82385000 & -0.87602600 \\
\hline $\mathrm{C}$ & -0.41130200 & 1.03100500 & 2.90007600 \\
\hline $\mathrm{C}$ & -1.64715500 & 0.35205200 & 2.62050400 \\
\hline $\mathrm{C}$ & -2.47762900 & 1.20620700 & 1.85220200 \\
\hline $\mathrm{C}$ & -1.78060200 & 2.41669700 & 1.63366200 \\
\hline $\mathrm{C}$ & -0.51354700 & 2.31710100 & 2.26267400 \\
\hline $\mathrm{H}$ & 1.00101100 & -2.11053100 & -0.98678700 \\
\hline $\mathrm{H}$ & 0.21440700 & -1.06422400 & 0.93767900 \\
\hline $\mathrm{H}$ & 0.27208500 & -0.13757600 & -0.99654200 \\
\hline $\mathrm{H}$ & -1.73916400 & -0.14985800 & -0.46946900 \\
\hline $\mathrm{H}$ & 1.35816000 & 1.00504400 & 0.39842500 \\
\hline $\mathrm{H}$ & -3.45129800 & 0.95421100 & 1.45130600 \\
\hline $\mathrm{H}$ & -1.90211000 & -0.64693200 & 2.94914500 \\
\hline $\mathrm{H}$ & -2.12311700 & 3.24727500 & 1.02843700 \\
\hline $\mathrm{H}$ & 0.25208200 & 3.08327200 & 2.26201600 \\
\hline $\mathrm{H}$ & 2.25378500 & 1.05857800 & 3.62002200 \\
\hline $\mathrm{H}$ & 0.70397600 & 0.81918300 & 5.43399400 \\
\hline $\mathrm{H}$ & 1.07927300 & -1.03923200 & 3.96454500 \\
\hline $\mathrm{H}$ & -0.83938600 & -3.39027500 & 1.99960500 \\
\hline $\mathrm{H}$ & -3.08156300 & -2.07360000 & 1.33134000 \\
\hline $\mathrm{H}$ & 0.07119900 & -4.65559000 & -0.18671600 \\
\hline $\mathrm{H}$ & -1.59383100 & -4.12823500 & -2.21598300 \\
\hline $\mathrm{H}$ & -5.30746400 & -3.32780300 & -1.74370700 \\
\hline $\mathrm{H}$ & -4.81102900 & -1.09479400 & -0.99720100 \\
\hline $\mathrm{H}$ & -3.86898800 & -1.94261800 & -3.06991800 \\
\hline $\mathrm{H}$ & 3.39501200 & -0.26134400 & -3.78351900 \\
\hline $\mathrm{H}$ & 3.64401000 & 1.85403200 & -2.61447700 \\
\hline $\mathrm{H}$ & 5.57547300 & 0.51634100 & -3.12765100 \\
\hline $\mathrm{H}$ & 4.09703400 & 1.22205800 & 0.43489700 \\
\hline $\mathrm{H}$ & 3.83769200 & -0.71174800 & 2.27119700 \\
\hline $\mathrm{H}$ & 3.55720700 & -3.05829700 & 0.97758900 \\
\hline $\mathrm{H}$ & 3.64322200 & -2.56638800 & -1.65738200 \\
\hline $\mathrm{C}$ & -0.36449800 & 4.94935100 & -0.87900700 \\
\hline $\mathrm{C}$ & -1.72347400 & 5.18238100 & -1.10528700 \\
\hline $\mathrm{C}$ & -2.51866600 & 4.19397900 & -1.69474200 \\
\hline $\mathrm{C}$ & -1.95341500 & 2.97021300 & -2.05901300 \\
\hline $\mathrm{C}$ & -0.58999600 & 2.73641400 & -1.83835000 \\
\hline $\mathrm{C}$ & 0.20445100 & 3.72625600 & -1.24636900 \\
\hline $\mathrm{H}$ & 0.25151000 & 5.72112400 & -0.42508400 \\
\hline $\mathrm{H}$ & -2.16345800 & 6.13627700 & -0.82598600 \\
\hline $\mathrm{H}$ & -3.57459000 & 4.37990700 & -1.87217100 \\
\hline $\mathrm{H}$ & -2.56645100 & 2.19839000 & -2.51577700 \\
\hline $\mathrm{H}$ & -0.14063300 & 1.79967700 & -2.15773400 \\
\hline $\mathrm{H}$ & 1.26213800 & 3.54174700 & -1.08214500 \\
\hline
\end{tabular}




$\begin{array}{llll}\mathrm{Ti} & -0.77303800 & -1.70434100 & -0.58073100 \\ \mathrm{H} & -1.31257500 & -1.14531900 & -2.40018400 \\ \mathrm{H} & -0.70580100 & -1.60968600 & -2.56340300\end{array}$

\begin{tabular}{|c|c|c|c|}
\hline & & & \\
\hline $\mathrm{Ti}$ & -1.52273500 & 0.61728800 & -0.69094600 \\
\hline $\mathrm{Ti}$ & 0.48230400 & 0.34246000 & 1.25736000 \\
\hline $\mathrm{Si}$ & 4.37825600 & -1.43761200 & -1.69127600 \\
\hline $\mathrm{Si}$ & -4.36159600 & -1.75248100 & -1.32102300 \\
\hline $\mathrm{Si}$ & -0.58531500 & 3.72788400 & 2.23697100 \\
\hline $\mathrm{C}$ & 3.24289800 & -0.03628400 & -2.15614400 \\
\hline $\mathrm{C}$ & 3.16037600 & 1.24456300 & -1.50608600 \\
\hline $\mathrm{C}$ & 2.23287700 & 2.05459500 & -2.20467400 \\
\hline $\mathrm{C}$ & 1.72319600 & 1.30722900 & -3.29271300 \\
\hline $\mathrm{C}$ & 2.32991200 & 0.02597700 & -3.26737200 \\
\hline $\mathrm{C}$ & -3.74441200 & 0.00545100 & -1.28747700 \\
\hline $\mathrm{C}$ & -3.82745100 & 0.92456800 & -0.19384000 \\
\hline $\mathrm{C}$ & -3.33861700 & 2.19039700 & -0.60806000 \\
\hline $\mathrm{C}$ & -2.93703100 & 2.08013000 & -1.96154500 \\
\hline $\mathrm{C}$ & -3.17844300 & 0.74463200 & -2.37698600 \\
\hline $\mathrm{C}$ & 0.58527400 & 2.30113900 & 2.47256800 \\
\hline $\mathrm{C}$ & 1.87973400 & 2.13851700 & 1.87107600 \\
\hline $\mathrm{C}$ & 2.50451300 & 0.99597000 & 2.42575600 \\
\hline $\mathrm{C}$ & 1.62024400 & 0.42623400 & 3.36996800 \\
\hline $\mathrm{C}$ & 0.43737300 & 1.21162200 & 3.40101200 \\
\hline $\mathrm{H}$ & -0.54311000 & 0.00994100 & -2.13952800 \\
\hline $\mathrm{H}$ & 0.16849700 & 1.43070500 & -0.33142700 \\
\hline $\mathrm{H}$ & -0.24106700 & -0.70971900 & -0.21506200 \\
\hline $\mathrm{H}$ & 1.75653000 & -0.60486500 & 0.27901700 \\
\hline $\mathrm{H}$ & -1.40068300 & 0.41011300 & 1.15146900 \\
\hline $\mathrm{H}$ & 3.46663200 & 0.59293900 & 2.13625600 \\
\hline $\mathrm{H}$ & 2.30764600 & 2.78502300 & 1.11628600 \\
\hline $\mathrm{H}$ & 1.80195800 & -0.47552800 & 3.94247100 \\
\hline $\mathrm{H}$ & -0.42582200 & 1.03541600 & 4.03187000 \\
\hline $\mathrm{H}$ & -1.98637000 & 3.30460600 & 2.50320400 \\
\hline $\mathrm{H}$ & -0.27346100 & 4.84461300 & 3.17668500 \\
\hline $\mathrm{H}$ & -0.48537400 & 4.27028800 & 0.85565200 \\
\hline $\mathrm{H}$ & 1.92543900 & 3.05478400 & -1.92541900 \\
\hline $\mathrm{H}$ & 3.71133700 & 1.53420900 & -0.62117100 \\
\hline $\mathrm{H}$ & 0.96270800 & 1.63597700 & -3.98950700 \\
\hline $\mathrm{H}$ & 2.13238800 & -0.77513300 & -3.97071600 \\
\hline $\mathrm{H}$ & 5.58034200 & -1.48349600 & -2.57369800 \\
\hline $\mathrm{H}$ & 4.84479700 & -1.26288100 & -0.29098300 \\
\hline $\mathrm{H}$ & 3.68887400 & -2.75040500 & -1.82617900 \\
\hline $\mathrm{H}$ & -3.51010200 & -2.59185200 & -2.20517100 \\
\hline $\mathrm{H}$ & -4.35392500 & -2.31587800 & 0.05595600 \\
\hline
\end{tabular}




$\begin{array}{lccc}\mathrm{H} & -5.75957700 & -1.83095600 & -1.83236600 \\ \mathrm{H} & -4.18873900 & 0.68906800 & 0.79993900 \\ \mathrm{H} & -3.24918900 & 3.07137300 & 0.01563100 \\ \mathrm{H} & -2.49235400 & 2.86457600 & -2.56194400 \\ \mathrm{H} & -2.95861400 & 0.34561300 & -3.35981700 \\ \mathrm{C} & -1.51196500 & -2.93957900 & 1.98957900 \\ \mathrm{C} & -0.36300200 & -2.49789600 & 2.65847300 \\ \mathrm{C} & -1.41933300 & -3.96505600 & 1.04832100 \\ \mathrm{H} & -2.47384100 & -2.48439600 & 2.20754500 \\ \mathrm{C} & 0.87773800 & -3.08134700 & 2.37575400 \\ \mathrm{H} & -0.44622500 & -1.72869300 & 3.42243700 \\ \mathrm{C} & -0.17935600 & -4.54972900 & 0.77061200 \\ \mathrm{H} & -2.31118900 & -4.31037900 & 0.53293500 \\ \mathrm{C} & 0.96809400 & -4.10785700 & 1.43045600 \\ \mathrm{H} & 1.77011000 & -2.74898200 & 2.89844800 \\ \mathrm{H} & -0.11035400 & -5.35175900 & 0.04034000 \\ \mathrm{H} & 1.93117700 & -4.56222500 & 1.21438700 \\ \mathrm{Ti} & 1.12157500 & 0.11649300 & -1.29364700 \\ \mathrm{H} & 0.19890700 & -2.72599400 & -2.71937400 \\ \mathrm{H} & 0.76295000 & -3.21212700 & -2.71651600\end{array}$

$\begin{array}{lrcc}\mathbf{A 5}^{\mathbf{T}} & & & \\ \mathrm{Ti} & -1.45232200 & 0.62192100 & -0.75520800 \\ \mathrm{Ti} & 0.54504300 & 0.42608000 & 1.21689600 \\ \mathrm{Si} & 4.22733200 & -2.04384300 & -1.41925500 \\ \mathrm{Si} & -4.37134300 & -1.65300900 & -1.43274600 \\ \mathrm{Si} & -0.22134700 & 3.97702700 & 1.79141300 \\ \mathrm{C} & 3.23681200 & -0.63452200 & -2.12850900 \\ \mathrm{C} & 3.28115000 & 0.73983700 & -1.70559900 \\ \mathrm{C} & 2.42150100 & 1.50139400 & -2.53392500 \\ \mathrm{C} & 1.83042000 & 0.62933300 & -3.47899300 \\ \mathrm{C} & 2.31941900 & -0.67929300 & -3.23549700 \\ \mathrm{C} & -3.68553800 & 0.08084900 & -1.39956200 \\ \mathrm{C} & -3.76903600 & 1.00997300 & -0.31807600 \\ \mathrm{C} & -3.21606900 & 2.25182000 & -0.72434700 \\ \mathrm{C} & -2.77710100 & 2.11744500 & -2.06474200 \\ \mathrm{C} & -3.05576500 & 0.78818400 & -2.47724100 \\ \mathrm{C} & 0.83275300 & 2.49643100 & 2.18758800 \\ \mathrm{C} & 2.09953400 & 2.15742100 & 1.60045000 \\ \mathrm{C} & 2.63891600 & 1.04153200 & 2.28347800 \\ \mathrm{C} & 1.72757500 & 0.66202200 & 3.29518400 \\ \mathrm{C} & 0.61261300 & 1.54073300 & 3.24086600 \\ \mathrm{H} & -0.52526400 & -0.18278400 & -2.13191600 \\ \mathrm{H} & 0.30119200 & 1.34888400 & -0.49823000 \\ \mathrm{H} & -0.26689200 & -0.73338500 & -0.14032000 \\ \mathrm{H} & 1.74326100 & -0.70633600 & 0.34323000\end{array}$




$\begin{array}{lrrr}\mathrm{H} & -1.32930100 & 0.63113500 & 1.09510100 \\ \mathrm{H} & 3.56054600 & 0.53006700 & 2.03665700 \\ \mathrm{H} & 2.56389400 & 2.67162100 & 0.76933700 \\ \mathrm{H} & 1.84618600 & -0.17643300 & 3.97158900 \\ \mathrm{H} & -0.25010900 & 1.51305900 & 3.89597200 \\ \mathrm{H} & -1.64579800 & 3.70917500 & 2.12637000 \\ \mathrm{H} & 0.20182600 & 5.17047500 & 2.58129600 \\ \mathrm{H} & -0.10943400 & 4.33570600 & 0.35226700 \\ \mathrm{H} & 2.21129400 & 2.55891600 & -2.43053800 \\ \mathrm{H} & 3.86855600 & 1.12331500 & -0.88168700 \\ \mathrm{H} & 1.09372000 & 0.90359800 & -4.22327700 \\ \mathrm{H} & 2.04097600 & -1.56658900 & -3.79331800 \\ \mathrm{H} & 5.40315900 & -2.37400900 & -2.27583100 \\ \mathrm{H} & 4.73144300 & -1.67679100 & -0.07013200 \\ \mathrm{H} & 3.39842900 & -3.27753500 & -1.32237800 \\ \mathrm{H} & -3.47946600 & -2.56859500 & -2.19251700 \\ \mathrm{H} & -4.52562300 & -2.15001400 & -0.04007100 \\ \mathrm{H} & -5.71283200 & -1.68899500 & -2.08177500 \\ \mathrm{H} & -4.16575600 & 0.79462000 & 0.66680200 \\ \mathrm{H} & -3.10927500 & 3.13164000 & -0.10162800 \\ \mathrm{H} & -2.28885700 & 2.88150900 & -2.65739400 \\ \mathrm{H} & -2.82486000 & 0.37337800 & -3.45082300 \\ \mathrm{C} & -1.85002600 & -2.50801000 & 2.59579000 \\ \mathrm{C} & -0.57433200 & -2.09185200 & 3.00038900 \\ \mathrm{C} & -1.98625300 & -3.57429900 & 1.70847800 \\ \mathrm{H} & -2.73082400 & -2.00214200 & 2.98146400 \\ \mathrm{C} & 0.56218700 & -2.74328100 & 2.50695700 \\ \mathrm{H} & -0.47448600 & -1.28870800 & 3.72558900 \\ \mathrm{C} & -0.84881900 & -4.22551500 & 1.21589700 \\ \mathrm{H} & -2.97530900 & -3.90139200 & 1.40048600 \\ \mathrm{C} & 0.42201200 & -3.81063500 & 1.61129300 \\ \mathrm{H} & 1.55290700 & -2.43775800 & 2.83023000 \\ \mathrm{H} & -0.95804500 & -5.05920500 & 0.52710300 \\ \mathrm{H} & 1.30517300 & -4.31561200 & 1.22972800 \\ & & -0.13848600 & -1.30134400\end{array}$

$\begin{array}{lccc}\text { TS5 }^{\mathbf{T}} & & & \\ \mathrm{Ti} & -1.65511200 & 0.38817500 & -0.29791900 \\ \mathrm{Ti} & 0.34279900 & -1.55403200 & -0.19108400 \\ \mathrm{Ti} & 1.06380700 & 1.22298600 & 0.72581600 \\ \mathrm{Si} & 3.87138800 & -2.24444800 & -1.31133500 \\ \mathrm{Si} & -4.98378400 & -0.86971600 & 0.58912300 \\ \mathrm{Si} & 1.08435400 & 3.28917200 & -2.39201500 \\ \mathrm{C} & 2.10272600 & -2.83315300 & -1.21498800 \\ \mathrm{C} & 1.07367900 & -2.60926700 & -2.18075800 \\ \mathrm{C} & -0.11135500 & -3.25631000 & -1.74960900\end{array}$




\begin{tabular}{|c|c|c|c|}
\hline $\mathrm{C}$ & 0.16320000 & -3.89100500 & -0.50671400 \\
\hline $\mathrm{C}$ & 1.51966200 & -3.62987700 & -0.18267700 \\
\hline $\mathrm{C}$ & -3.96810800 & 0.22226300 & -0.53034000 \\
\hline $\mathrm{C}$ & -3.65836200 & 1.61173100 & -0.35294000 \\
\hline $\mathrm{C}$ & -2.91458000 & 2.06924300 & -1.46908200 \\
\hline $\mathrm{C}$ & -2.74065500 & 0.97736400 & -2.35356100 \\
\hline $\mathrm{C}$ & -3.38231300 & -0.15207500 & -1.78535800 \\
\hline $\mathrm{C}$ & 1.79684800 & 2.96028300 & -0.70344600 \\
\hline $\mathrm{C}$ & 2.91250900 & 2.11915600 & -0.38586800 \\
\hline $\mathrm{C}$ & 3.20912300 & 2.24391300 & 0.99612100 \\
\hline $\mathrm{C}$ & 2.28048500 & 3.16137200 & 1.55417700 \\
\hline $\mathrm{C}$ & 1.41889200 & 3.59526000 & 0.52382700 \\
\hline $\mathrm{H}$ & -1.51160400 & -1.43935600 & 0.01358400 \\
\hline $\mathrm{H}$ & -0.22812200 & -0.09212000 & 0.71575500 \\
\hline $\mathrm{H}$ & 1.69341100 & -0.30128400 & -0.15637300 \\
\hline $\mathrm{H}$ & -0.28949200 & -0.25203600 & -1.37621000 \\
\hline $\mathrm{H}$ & -0.68234400 & 1.82106800 & 0.33000000 \\
\hline $\mathrm{H}$ & 3.99702800 & 1.72494200 & 1.52730400 \\
\hline $\mathrm{H}$ & 3.44378300 & 1.48675800 & -1.08614000 \\
\hline $\mathrm{H}$ & 2.21868600 & 3.45038700 & 2.59683700 \\
\hline $\mathrm{H}$ & 0.58382900 & 4.27419200 & 0.64673500 \\
\hline $\mathrm{H}$ & -0.27408700 & 3.87987100 & -2.26227300 \\
\hline $\mathrm{H}$ & 1.92390500 & 4.26898900 & -3.14151200 \\
\hline $\mathrm{H}$ & 1.01428200 & 2.05009700 & -3.20948100 \\
\hline $\mathrm{H}$ & -1.06689500 & -3.23851300 & -2.25724900 \\
\hline $\mathrm{H}$ & 1.17234200 & -2.00835700 & -3.07633600 \\
\hline $\mathrm{H}$ & -0.54231900 & -4.45666500 & 0.08938200 \\
\hline $\mathrm{H}$ & 2.02983600 & -3.96895300 & 0.71209000 \\
\hline $\mathrm{H}$ & 4.73963900 & -3.28351300 & -1.93534600 \\
\hline $\mathrm{H}$ & 3.96727000 & -1.01540200 & -2.14016400 \\
\hline $\mathrm{H}$ & 4.40541400 & -1.97780600 & 0.05147500 \\
\hline $\mathrm{H}$ & -4.70343300 & -2.29749400 & 0.28894400 \\
\hline $\mathrm{H}$ & -4.66894000 & -0.59216200 & 2.01807900 \\
\hline $\mathrm{H}$ & -6.44330600 & -0.63182300 & 0.40358900 \\
\hline $\mathrm{H}$ & -3.92780600 & 2.21458400 & 0.50766900 \\
\hline $\mathrm{H}$ & -2.50421000 & 3.06226000 & -1.59645300 \\
\hline $\mathrm{H}$ & -2.17755100 & 0.99103200 & -3.27818400 \\
\hline $\mathrm{H}$ & -3.39938900 & -1.14701200 & -2.21256300 \\
\hline $\mathrm{C}$ & 1.47780600 & 0.17663500 & 2.69044000 \\
\hline $\mathrm{C}$ & 1.35821700 & -1.23825200 & 2.42254700 \\
\hline $\mathrm{C}$ & 0.13894200 & -1.89647900 & 2.52615700 \\
\hline $\mathrm{C}$ & -1.03090200 & -1.18345900 & 2.95481400 \\
\hline $\mathrm{C}$ & -0.95426300 & 0.17367800 & 3.17710800 \\
\hline $\mathrm{C}$ & 0.26673200 & 0.89668300 & 2.99028700 \\
\hline $\mathrm{H}$ & 2.44404300 & 0.54803500 & 3.01668800 \\
\hline $\mathrm{H}$ & 2.26694900 & -1.80789400 & 2.24705700 \\
\hline
\end{tabular}




$\begin{array}{cccc}\mathrm{H} & 0.09812000 & -2.97706500 & 2.42064200 \\ \mathrm{H} & -1.96335300 & -1.71989500 & 3.09747500 \\ \mathrm{H} & -1.83168100 & 0.71760100 & 3.52197500 \\ \mathrm{H} & 0.32600100 & 1.90437800 & 3.39148400\end{array}$

$\mathbf{A 6}^{\mathrm{T}}$

Ti

Ti

Ti

$\mathrm{Si}$

$\mathrm{Si}$

$\mathrm{Si}$

C

C

C

C

C

C

C

C

C

C

C

C

C

C

C

$\mathrm{H}$

$\mathrm{H}$

$\mathrm{H}$

$\mathrm{H}$

$\mathrm{H}$

$\mathrm{H}$

$\mathrm{H}$

$\mathrm{H}$

$\mathrm{H}$

$\mathrm{H}$

$\mathrm{H}$

$\mathrm{H}$

$\mathrm{H}$

$\mathrm{H}$

$\mathrm{H}$

$\mathrm{H}$

$\mathrm{H}$

$\mathrm{H}$

$\mathrm{H}$

$\begin{array}{ccc}1.20654500 & -1.23937600 & 0.14304600 \\ -1.59561100 & -0.97263900 & 0.25998900 \\ 0.02377900 & 1.51428700 & -0.33266900 \\ -4.98930500 & 0.59756200 & 0.05651600 \\ 4.80719100 & -0.63120100 & -0.70093900 \\ 1.97283000 & 2.86789500 & 2.46998100 \\ -3.92369300 & -0.80815000 & 0.66051200 \\ -3.23515400 & -0.87004700 & 1.91890600 \\ -2.61758100 & -2.13834500 & 2.04242400 \\ -2.91172000 & -2.88427400 & 0.86826200 \\ -3.70799100 & -2.07329800 & 0.02714800 \\ 3.52924900 & -1.62961900 & 0.21873100 \\ 3.06582500 & -1.38904600 & 1.55656300 \\ 2.19686900 & -2.43898500 & 1.93777100 \\ 2.10464200 & -3.34865800 & 0.85030100 \\ 2.91490900 & -2.85573300 & -0.19889000 \\ 0.90177200 & 3.22522200 & 0.98442800 \\ -0.52512500 & 3.36431900 & 0.96289900 \\ -0.93144500 & 3.72263700 & -0.34909000 \\ 0.23267400 & 3.81649400 & -1.15023100 \\ 1.35156900 & 3.50883300 & -0.34700700 \\ -0.31317600 & -2.27965400 & 0.02400100 \\ -0.14210700 & -0.26341600 & -0.45129500 \\ -1.57446600 & 0.87671000 & 0.37278400 \\ -0.10843500 & -0.74724500 & 1.43772900 \\ 1.54123300 & 0.58444800 & 0.22342900 \\ -1.95144900 & 3.86544100 & -0.68329600 \\ -1.18880500 & 3.20953000 & 1.80455600 \\ 0.25492500 & 4.02977900 & -2.21223100 \\ 2.37908100 & 3.46065700 & -0.68730200 \\ 3.29702500 & 2.35397300 & 2.03505000 \\ 2.20287700 & 4.11013500 & 3.26229100 \\ 1.32101300 & 1.87969400 & 3.36949600 \\ -1.99540100 & -2.46642700 & 2.86507400 \\ -3.17490400 & -0.06629900 & 2.64229600 \\ -2.55541000 & -3.88123800 & 0.64158800 \\ -4.07634900 & -2.35738900 & -0.95238200 \\ -6.42366600 & 0.36171800 & 0.38639900 \\ -4.57331900 & 1.87067400 & 0.69880400 \\ -4.89579600 & 0.73157600 & -1.42353300\end{array}$

S90 


$\begin{array}{lrrr}\mathrm{H} & 4.55574400 & -0.68839100 & -2.16775300 \\ \mathrm{H} & 4.78683400 & 0.78322700 & -0.24995600 \\ \mathrm{H} & 6.17681700 & -1.17122800 & -0.46879200 \\ \mathrm{H} & 3.31868900 & -0.52870300 & 2.16329800 \\ \mathrm{H} & 1.66046400 & -2.51207700 & 2.87512900 \\ \mathrm{H} & 1.48867800 & -4.23819900 & 0.81538500 \\ \mathrm{H} & 3.03950700 & -3.32429600 & -1.16870600 \\ \mathrm{C} & -0.85663100 & 1.06108200 & -2.32169700 \\ \mathrm{C} & -1.66258200 & -0.14643400 & -2.36034700 \\ \mathrm{C} & -1.10432400 & -1.40737900 & -2.35179400 \\ \mathrm{C} & 0.35212700 & -1.55373400 & -2.43717100 \\ \mathrm{C} & 1.14477700 & -0.42973700 & -2.51767200 \\ \mathrm{C} & 0.60471700 & 0.91280900 & -2.39403400 \\ \mathrm{H} & -1.32728000 & 1.95394900 & -2.72477600 \\ \mathrm{H} & -2.73989700 & -0.03876100 & -2.46689500 \\ \mathrm{H} & -1.72699400 & -2.28968300 & -2.46629700 \\ \mathrm{H} & 0.77050100 & -2.54101300 & -2.60877600 \\ \mathrm{H} & 2.20271500 & -0.53708800 & -2.74627700 \\ \mathrm{H} & 1.20034100 & 1.70225900 & -2.84496700\end{array}$

TS6 $^{\mathrm{T}}$

$\mathrm{Ti}$

$\mathrm{Ti}$

Ti

Si

$\mathrm{Si}$

$\mathrm{Si}$

C

C

C

C

C

C

C

C

C

C

C

C

C

C

C

$\mathrm{H}$

$\mathrm{H}$

$\mathrm{H}$

$\mathrm{H}$

$\begin{array}{rcc}1.37962400 & -1.06067000 & 0.23895900 \\ -1.57179100 & -0.75453000 & 0.36403300 \\ 0.06814000 & 1.35887400 & -0.53820700 \\ -5.09180800 & -0.47263200 & -0.85629100 \\ 5.00233200 & -0.41896100 & -0.52653900 \\ 0.33032700 & 3.36478700 & 2.57781100 \\ -3.91306900 & -0.96605500 & 0.49838200 \\ -3.47367400 & -0.14338600 & 1.58907900 \\ -2.68373200 & -0.92278800 & 2.46942000 \\ -2.61744600 & -2.23819100 & 1.94398500 \\ -3.36287800 & -2.26798800 & 0.74062400 \\ 3.73838300 & -1.34022900 & 0.49079900 \\ 3.17251900 & -0.90440400 & 1.73491200 \\ 2.30830300 & -1.91115200 & 2.22878800 \\ 2.32709200 & -2.99013600 & 1.30085600 \\ 3.19980000 & -2.64150400 & 0.24547700 \\ 0.29272400 & 3.36715500 & 0.71269900 \\ -0.86547800 & 3.48707200 & -0.11759500 \\ -0.46212800 & 3.48185300 & -1.47628300 \\ 0.95664700 & 3.36752600 & -1.50715900 \\ 1.41510000 & 3.30012400 & -0.17124200 \\ -0.16080800 & -1.89534800 & 0.79766000 \\ -0.07508400 & -0.36834100 & -0.47843600 \\ -1.69034700 & 0.96347100 & -0.25756500 \\ -0.27606200 & 0.47374100 & 1.18101100\end{array}$

S91 


$\begin{array}{lrrc}\mathrm{H} & 1.64134200 & 0.74218700 & 0.12695600 \\ \mathrm{H} & -1.11916700 & 3.55523100 & -2.33322900 \\ \mathrm{H} & -1.88752800 & 3.54484400 & 0.23421900 \\ \mathrm{H} & 1.57633600 & 3.32851700 & -2.39424100 \\ \mathrm{H} & 2.44807500 & 3.18584500 & 0.13236000 \\ \mathrm{H} & 1.42660500 & 2.49909400 & 3.08741600 \\ \mathrm{H} & 0.57203500 & 4.74033400 & 3.10285100 \\ \mathrm{H} & -0.97375600 & 2.90094000 & 3.11914600 \\ \mathrm{H} & -2.18239900 & -0.56542000 & 3.36011100 \\ \mathrm{H} & -3.68880400 & 0.91148800 & 1.70602400 \\ \mathrm{H} & -2.05845100 & -3.06384200 & 2.36557200 \\ \mathrm{H} & -3.48650500 & -3.13419500 & 0.10047200 \\ \mathrm{H} & -6.50495800 & -0.77082800 & -0.48880300 \\ \mathrm{H} & -4.98606200 & 0.98501700 & -1.12909700 \\ \mathrm{H} & -4.79135900 & -1.22708400 & -2.10529600 \\ \mathrm{H} & 4.75328500 & -0.61918300 & -1.98116100 \\ \mathrm{H} & 4.94688500 & 1.03119500 & -0.20871400 \\ \mathrm{H} & 6.38072500 & -0.90665900 & -0.24097900 \\ \mathrm{H} & 3.35450300 & 0.05486500 & 2.20375700 \\ \mathrm{H} & 1.71398500 & -1.85834600 & 3.13217600 \\ \mathrm{H} & 1.74493900 & -3.90033600 & 1.37245900 \\ \mathrm{H} & 3.40596500 & -3.25467800 & -0.62480700 \\ \mathrm{C} & -0.49235400 & 0.46871400 & -2.61145500 \\ \mathrm{C} & -1.40063000 & -0.61806300 & -2.42033700 \\ \mathrm{C} & -0.98948500 & -1.86577000 & -1.95108400 \\ \mathrm{C} & 0.45288000 & -2.14549800 & -1.90805600 \\ \mathrm{C} & 1.36203900 & -1.14638700 & -2.21848800 \\ \mathrm{C} & 0.93475400 & 0.23135900 & -2.40424100 \\ \mathrm{H} & -0.82240900 & 1.29749400 & -3.22869400 \\ \mathrm{H} & -2.44708900 & -0.46439300 & -2.67174500 \\ \mathrm{H} & -1.68705500 & -2.69709400 & -1.92394100 \\ \mathrm{H} & 0.78030700 & -3.17911400 & -1.83859200 \\ \mathrm{H} & 2.40145400 & -1.40202400 & -2.40760200 \\ & 1.65190000 & 0.89355900 & -2.87877100\end{array}$

\section{A $7^{\mathrm{T}}$}

Ti

Ti

$\mathrm{Ti}$

$\mathrm{Si}$

$\mathrm{Si}$

$\mathrm{Si}$

C

C

C

C

$\begin{array}{ccc}-1.49073400 & -0.98280500 & -0.27410300 \\ 1.59787600 & -0.72285600 & -0.39470900 \\ -0.05434800 & 1.32217600 & 0.55970200 \\ 4.97391600 & -0.95440000 & 1.13885800 \\ -5.13243300 & -0.38180300 & 0.48277100 \\ 0.54437000 & 3.61479200 & -2.29601300 \\ 3.90895600 & -1.14575300 & -0.37603700 \\ 3.64365000 & -0.14128600 & -1.36562400 \\ 2.87561700 & -0.71236000 & -2.41126700 \\ 2.65251100 & -2.07536500 & -2.09127300\end{array}$




\begin{tabular}{|c|c|c|c|}
\hline $\mathrm{C}$ & 3.27755900 & -2.34381200 & -0.85047500 \\
\hline $\mathrm{C}$ & -3.86232200 & -1.15307200 & -0.64243400 \\
\hline $\mathrm{C}$ & -3.27116900 & -0.54256100 & -1.79670700 \\
\hline $\mathrm{C}$ & -2.40357300 & -1.46703900 & -2.41879200 \\
\hline $\mathrm{C}$ & -2.44745100 & -2.67565300 & -1.66437300 \\
\hline $\mathrm{C}$ & -3.34294000 & -2.48468200 & -0.58741600 \\
\hline $\mathrm{C}$ & 0.06091500 & 3.44019500 & -0.50126100 \\
\hline $\mathrm{C}$ & 0.93962300 & 3.46971700 & 0.62909600 \\
\hline $\mathrm{C}$ & 0.17111800 & 3.35222200 & 1.81218100 \\
\hline $\mathrm{C}$ & -1.19530700 & 3.24356400 & 1.43639900 \\
\hline $\mathrm{C}$ & -1.26145900 & 3.30477100 & 0.02338000 \\
\hline $\mathrm{H}$ & 0.07662700 & -1.68332400 & -1.03287800 \\
\hline $\mathrm{H}$ & 0.05443300 & -0.35706300 & 0.30601200 \\
\hline $\mathrm{H}$ & 1.71612200 & 0.87283800 & 0.57513400 \\
\hline $\mathrm{H}$ & 0.70687000 & 0.85310100 & -1.06307500 \\
\hline $\mathrm{H}$ & -1.40644700 & 0.82921300 & -0.51901500 \\
\hline $\mathrm{H}$ & 0.56120500 & 3.33929300 & 2.82175400 \\
\hline $\mathrm{H}$ & 2.01841800 & 3.54949200 & 0.58486700 \\
\hline $\mathrm{H}$ & -2.03638100 & 3.11981500 & 2.10773600 \\
\hline $\mathrm{H}$ & -2.16730200 & 3.23651000 & -0.56512800 \\
\hline $\mathrm{H}$ & -0.36988300 & 2.83444900 & -3.16901800 \\
\hline $\mathrm{H}$ & 0.45835900 & 5.04257300 & -2.72206400 \\
\hline $\mathrm{H}$ & 1.94861900 & 3.17274600 & -2.49623500 \\
\hline $\mathrm{H}$ & 2.49085100 & -0.18891600 & -3.27745500 \\
\hline $\mathrm{H}$ & 3.95373300 & 0.89499800 & -1.30851900 \\
\hline $\mathrm{H}$ & 2.06467800 & -2.77590400 & -2.67079200 \\
\hline $\mathrm{H}$ & 3.26759100 & -3.29847300 & -0.33705000 \\
\hline $\mathrm{H}$ & 6.37803200 & -1.38557900 & 0.88715000 \\
\hline $\mathrm{H}$ & 5.00031300 & 0.46889500 & 1.56882700 \\
\hline $\mathrm{H}$ & 4.44921300 & -1.79107500 & 2.25446600 \\
\hline $\mathrm{H}$ & -4.86982100 & -0.74434000 & 1.90415400 \\
\hline $\mathrm{H}$ & -5.09305300 & 1.09814900 & 0.34269100 \\
\hline $\mathrm{H}$ & -6.50970800 & -0.84427400 & 0.15481000 \\
\hline $\mathrm{H}$ & -3.44071100 & 0.47627900 & -2.12158400 \\
\hline $\mathrm{H}$ & -1.78947700 & -1.28126500 & -3.29043000 \\
\hline $\mathrm{H}$ & -1.87603600 & -3.57267800 & -1.86759900 \\
\hline $\mathrm{H}$ & -3.57871300 & -3.22357000 & 0.16990200 \\
\hline $\mathrm{C}$ & 0.04908200 & 0.28119000 & 2.59729200 \\
\hline $\mathrm{C}$ & 1.12465900 & -0.62479000 & 2.44598600 \\
\hline $\mathrm{C}$ & 0.98093700 & -1.87735200 & 1.81120100 \\
\hline $\mathrm{C}$ & -0.38414000 & -2.37612600 & 1.67559500 \\
\hline $\mathrm{C}$ & -1.47504800 & -1.56274600 & 1.93077800 \\
\hline $\mathrm{C}$ & -1.28547900 & -0.10879300 & 2.07857100 \\
\hline $\mathrm{H}$ & 0.13488900 & 1.05158600 & 3.35642500 \\
\hline $\mathrm{H}$ & 2.09050700 & -0.34857300 & 2.86181800 \\
\hline $\mathrm{H}$ & 1.79458600 & -2.59459700 & 1.85771600 \\
\hline
\end{tabular}




$\begin{array}{lrrl}\mathrm{H} & -0.53261600 & -3.44340200 & 1.52972800 \\ \mathrm{H} & -2.45472700 & -1.99904300 & 2.10141100 \\ \mathrm{H} & -2.13805600 & 0.42696400 & 2.489132000\end{array}$

\section{МЕCP}

\begin{tabular}{|c|c|c|c|}
\hline $\mathrm{Ti}$ & 1.56639800 & -0.77066700 & 0.22430100 \\
\hline $\mathrm{Ti}$ & -1.37096400 & -1.02256700 & 0.24897600 \\
\hline $\mathrm{Ti}$ & -0.16510700 & 1.27698200 & -0.49239900 \\
\hline $\mathrm{Si}$ & -4.93557100 & -0.67872300 & -0.84018300 \\
\hline $\mathrm{Si}$ & 5.20472300 & -0.05151700 & -0.50588700 \\
\hline $\mathrm{Si}$ & -0.95207200 & 3.36853700 & 2.49404600 \\
\hline $\mathrm{C}$ & -3.71906700 & -1.45147100 & 0.34159600 \\
\hline $\mathrm{C}$ & -3.29719400 & -0.91221200 & 1.60218200 \\
\hline $\mathrm{C}$ & -2.41680700 & -1.82783600 & 2.22995800 \\
\hline $\mathrm{C}$ & -2.28183900 & -2.94998700 & 1.36906800 \\
\hline $\mathrm{C}$ & -3.07879200 & -2.72383400 & 0.22076400 \\
\hline $\mathrm{C}$ & 3.93747000 & -0.79304300 & 0.63836500 \\
\hline $\mathrm{C}$ & 3.29053000 & -0.12018600 & 1.72549000 \\
\hline $\mathrm{C}$ & 2.46958500 & -1.03892400 & 2.42281200 \\
\hline $\mathrm{C}$ & 2.60016100 & -2.30164900 & 1.78364300 \\
\hline $\mathrm{C}$ & 3.49062800 & -2.15253600 & 0.69569400 \\
\hline $\mathrm{C}$ & -0.59098700 & 3.31504100 & 0.66359700 \\
\hline $\mathrm{C}$ & -1.55318200 & 3.21138900 & -0.39535400 \\
\hline $\mathrm{C}$ & -0.88445900 & 3.29023600 & -1.63749400 \\
\hline $\mathrm{C}$ & 0.50376700 & 3.42751200 & -1.37417300 \\
\hline $\mathrm{C}$ & 0.68020900 & 3.45620900 & 0.03097600 \\
\hline $\mathrm{H}$ & 0.20579300 & -1.94386400 & 0.65451500 \\
\hline $\mathrm{H}$ & 0.05455500 & -0.02814500 & 0.83040300 \\
\hline $\mathrm{H}$ & -1.77125000 & 0.39736900 & -0.81070700 \\
\hline $\mathrm{H}$ & -1.36893800 & 0.74657400 & 0.85626900 \\
\hline $\mathrm{H}$ & 1.42604400 & 1.05489700 & 0.37832400 \\
\hline $\mathrm{H}$ & -1.34919700 & 3.23096000 & -2.61338300 \\
\hline $\mathrm{H}$ & -2.61930700 & 3.07312400 & -0.26264500 \\
\hline $\mathrm{H}$ & 1.29339500 & 3.48360800 & -2.11398800 \\
\hline $\mathrm{H}$ & 1.63055300 & 3.54987300 & 0.54088100 \\
\hline $\mathrm{H}$ & 0.11512700 & 2.68343300 & 3.26989400 \\
\hline $\mathrm{H}$ & -1.02080600 & 4.77848700 & 2.97536100 \\
\hline $\mathrm{H}$ & -2.26512200 & 2.73071100 & 2.77186200 \\
\hline $\mathrm{H}$ & -1.92107800 & -1.68857400 & 3.18245300 \\
\hline $\mathrm{H}$ & -3.59248300 & 0.04993600 & 2.00174500 \\
\hline $\mathrm{H}$ & -1.65727100 & -3.81660200 & 1.54817400 \\
\hline $\mathrm{H}$ & -3.18186400 & -3.40444400 & -0.61629200 \\
\hline $\mathrm{H}$ & -6.30998700 & -1.22462100 & -0.65073000 \\
\hline $\mathrm{H}$ & -4.99509600 & 0.79070600 & -0.62026700 \\
\hline $\mathrm{H}$ & -4.54786900 & -0.94839700 & -2.25316400 \\
\hline $\mathrm{H}$ & 5.03348400 & -0.56190800 & -1.89571100 \\
\hline
\end{tabular}




$\begin{array}{lrrc}\mathrm{H} & 5.05987100 & 1.42923000 & -0.51770300 \\ \mathrm{H} & 6.59815500 & -0.37761100 & -0.09131200 \\ \mathrm{H} & 3.40771100 & 0.92811300 & 1.97021600 \\ \mathrm{H} & 1.84353800 & -0.81469000 & 3.27725100 \\ \mathrm{H} & 2.08419000 & -3.21125100 & 2.06303200 \\ \mathrm{H} & 3.78003400 & -2.94083900 & 0.00956700 \\ \mathrm{C} & 0.06476400 & 0.49452900 & -2.56580100 \\ \mathrm{C} & -0.90412100 & -0.54724200 & -2.50080100 \\ \mathrm{C} & -0.60356800 & -1.81978700 & -1.97363300 \\ \mathrm{C} & 0.79988500 & -2.17673300 & -1.80968700 \\ \mathrm{C} & 1.79191200 & -1.22156400 & -1.97008400 \\ \mathrm{C} & 1.39409900 & 0.18084800 & -2.01586800 \\ \mathrm{H} & -0.06163400 & 1.27043900 & -3.31448200 \\ \mathrm{H} & -1.88248600 & -0.37625500 & -2.94103800 \\ \mathrm{H} & -1.32531500 & -2.62275300 & -2.09391800 \\ \mathrm{H} & 1.05811200 & -3.22820900 & -1.71690900 \\ \mathrm{H} & 2.82764500 & -1.51015300 & -2.11468300 \\ \mathrm{H} & 2.18722600 & 0.88967200 & -2.24147700\end{array}$

\section{TS7 $^{\mathrm{T}}$}

Ti

Ti

$\mathrm{Ti}$

$\mathrm{Si}$

Si

$\mathrm{Si}$

C

C

C

C

C

C

C

C

C

C

C

C

C

C

C

$\mathrm{H}$

$\mathrm{H}$

$\mathrm{H}$

$\mathrm{H}$

$\mathrm{H}$

$\begin{array}{ccc}-1.26582800 & -1.06652100 & -0.08816800 \\ 1.59672000 & -0.81934400 & -0.23463900 \\ 0.19437300 & 1.35330500 & 0.35109000 \\ 5.13502700 & 0.23821400 & 0.18967600 \\ -4.93105000 & -0.67358500 & 0.76041200 \\ -2.16927600 & 3.15926400 & -1.96150500 \\ 3.93869400 & -0.92180000 & -0.64594800 \\ 3.27707800 & -0.71048900 & -1.89835900 \\ 2.49765800 & -1.85125500 & -2.20930100 \\ 2.66701700 & -2.79172700 & -1.15882800 \\ 3.54631000 & -2.22490500 & -0.20361400 \\ -3.62983800 & -1.45796000 & -0.31378800 \\ -3.14450100 & -0.96389900 & -1.56576600 \\ -2.24095000 & -1.90254000 & -2.12114800 \\ -2.14784700 & -2.99905400 & -1.22407600 \\ -2.99073100 & -2.72630800 & -0.11965400 \\ -0.74689000 & 3.26352100 & -0.75602800 \\ 0.64185800 & 3.22543400 & -1.07424800 \\ 1.39382800 & 3.42723400 & 0.11300900 \\ 0.48132900 & 3.59717300 & 1.18259100 \\ -0.83009300 & 3.48184800 & 0.65632200 \\ 0.25084000 & -2.12815800 & -0.13380600 \\ 0.10813100 & -0.74350300 & -1.25461100 \\ 1.97776100 & 0.80966000 & 0.53342700 \\ 1.17181200 & 0.75290700 & -1.06466100 \\ -1.24851800 & 0.72598700 & -0.56954100\end{array}$

S95 


\begin{tabular}{|c|c|c|c|}
\hline $\mathrm{H}$ & 2.47377900 & 3.43020400 & 0.18765900 \\
\hline $\mathrm{H}$ & 1.05759800 & 3.05860900 & -2.05960200 \\
\hline $\mathrm{H}$ & 0.74320400 & 3.77891400 & 2.21656800 \\
\hline $\mathrm{H}$ & -1.74874500 & 3.54350700 & 1.22898300 \\
\hline $\mathrm{H}$ & -3.39119400 & 2.66272400 & -1.27869700 \\
\hline $\mathrm{H}$ & -2.47693500 & 4.51126700 & -2.51134600 \\
\hline $\mathrm{H}$ & -1.82797500 & 2.27415900 & -3.10463700 \\
\hline $\mathrm{H}$ & 1.86871900 & -1.97522100 & -3.08158800 \\
\hline $\mathrm{H}$ & 3.34591800 & 0.18695900 & -2.50095700 \\
\hline $\mathrm{H}$ & 2.19196200 & -3.76223300 & -1.09138100 \\
\hline $\mathrm{H}$ & 3.87181300 & -2.70374900 & 0.71314700 \\
\hline $\mathrm{H}$ & 6.54922100 & -0.10849600 & -0.13106200 \\
\hline $\mathrm{H}$ & 4.89639800 & 1.63464000 & -0.26184400 \\
\hline $\mathrm{H}$ & 4.98876100 & 0.16561700 & 1.67017400 \\
\hline $\mathrm{H}$ & -4.65027500 & -0.94782000 & 2.19906800 \\
\hline $\mathrm{H}$ & -4.95895900 & 0.79760800 & 0.54533600 \\
\hline $\mathrm{H}$ & -6.29329100 & -1.20566100 & 0.47293200 \\
\hline $\mathrm{H}$ & -3.41503800 & -0.01531500 & -2.01223400 \\
\hline $\mathrm{H}$ & -1.70218600 & -1.79296400 & -3.05375000 \\
\hline $\mathrm{H}$ & -1.52747700 & -3.87708200 & -1.35195800 \\
\hline $\mathrm{H}$ & -3.13008400 & -3.37737100 & 0.73665900 \\
\hline $\mathrm{C}$ & 0.10676000 & 0.88205700 & 2.51067900 \\
\hline $\mathrm{C}$ & 1.26114000 & 0.07385400 & 2.51539800 \\
\hline $\mathrm{C}$ & 1.20327400 & -1.29767300 & 2.15936100 \\
\hline $\mathrm{C}$ & -0.09608500 & -1.94431800 & 2.18254300 \\
\hline $\mathrm{C}$ & -1.25366900 & -1.19103200 & 2.19966400 \\
\hline $\mathrm{C}$ & -1.12578000 & 0.26031800 & 1.98438100 \\
\hline $\mathrm{H}$ & 0.07650100 & 1.76869700 & 3.13413100 \\
\hline $\mathrm{H}$ & 2.20094000 & 0.48949500 & 2.86901200 \\
\hline $\mathrm{H}$ & 2.08508300 & -1.91404100 & 2.30844200 \\
\hline $\mathrm{H}$ & -0.13527600 & -3.02442400 & 2.29526400 \\
\hline $\mathrm{H}$ & -2.20587200 & -1.63522600 & 2.47131400 \\
\hline $\mathrm{H}$ & -2.03963600 & 0.83126800 & 2.13803900 \\
\hline
\end{tabular}

$\begin{array}{rrrc}\mathbf{A 8}^{\mathbf{T}} & & & \\ \mathrm{Ti} & -1.16390500 & -1.18360100 & -0.12196900 \\ \mathrm{Ti} & 1.53261300 & -0.85767200 & -0.24567200 \\ \mathrm{Ti} & 0.24808400 & 1.37293400 & 0.35185600 \\ \mathrm{Si} & 5.02639800 & 0.38768300 & 0.16731300 \\ \mathrm{Si} & -4.82175800 & -0.57054500 & 0.86028600 \\ \mathrm{Si} & -2.22076300 & 3.06487500 & -2.00372400 \\ \mathrm{C} & 3.88740200 & -0.85727300 & -0.63050000 \\ \mathrm{C} & 3.23461000 & -0.72674100 & -1.89760400 \\ \mathrm{C} & 2.51576500 & -1.91504900 & -2.16911700 \\ \mathrm{C} & 2.71534400 & -2.80498400 & -1.07965300 \\ \mathrm{C} & 3.55325700 & -2.15880100 & -0.13974400\end{array}$




\begin{tabular}{|c|c|c|c|}
\hline $\mathrm{C}$ & -3.58887900 & -1.41964700 & -0.24791300 \\
\hline $\mathrm{C}$ & -3.12432900 & -0.95964300 & -1.51821100 \\
\hline $\mathrm{C}$ & -2.28894700 & -1.94890200 & -2.09014300 \\
\hline $\mathrm{C}$ & -2.22747100 & -3.04685700 & -1.18733200 \\
\hline $\mathrm{C}$ & -3.01882000 & -2.72182500 & -0.06205100 \\
\hline $\mathrm{C}$ & -0.81660600 & 3.22462200 & -0.78769500 \\
\hline $\mathrm{C}$ & 0.57768900 & 3.23686300 & -1.09847400 \\
\hline $\mathrm{C}$ & 1.31382900 & 3.50237700 & 0.08469700 \\
\hline $\mathrm{C}$ & 0.38658500 & 3.65327900 & 1.14555100 \\
\hline $\mathrm{C}$ & -0.91712000 & 3.47395800 & 0.61554100 \\
\hline $\mathrm{H}$ & 0.30025500 & -2.27365400 & -0.10424200 \\
\hline $\mathrm{H}$ & 0.16988300 & -1.04905200 & -1.40098300 \\
\hline $\mathrm{H}$ & 1.99294200 & 0.83302200 & 0.43783400 \\
\hline $\mathrm{H}$ & 1.07826700 & 0.64868100 & -1.11196600 \\
\hline $\mathrm{H}$ & -1.06312200 & 0.59311100 & -0.62733300 \\
\hline $\mathrm{H}$ & 2.39201000 & 3.56145400 & 0.16400400 \\
\hline $\mathrm{H}$ & 1.00454600 & 3.06565400 & -2.07884600 \\
\hline $\mathrm{H}$ & 0.63406000 & 3.86482600 & 2.17840300 \\
\hline $\mathrm{H}$ & -1.84097100 & 3.51590600 & 1.18178400 \\
\hline $\mathrm{H}$ & -3.43413200 & 2.53212900 & -1.33103900 \\
\hline $\mathrm{H}$ & -2.57464800 & 4.39782500 & -2.57249200 \\
\hline $\mathrm{H}$ & -1.84401900 & 2.18096300 & -3.13746200 \\
\hline $\mathrm{H}$ & 1.90350300 & -2.10339500 & -3.04124500 \\
\hline $\mathrm{H}$ & 3.26463600 & 0.14982200 & -2.53244700 \\
\hline $\mathrm{H}$ & 2.28459700 & -3.79289500 & -0.97855900 \\
\hline $\mathrm{H}$ & 3.89028300 & -2.58412000 & 0.79820300 \\
\hline $\mathrm{H}$ & 6.45311100 & 0.05874700 & -0.11344300 \\
\hline $\mathrm{H}$ & 4.75598900 & 1.74731500 & -0.36666600 \\
\hline $\mathrm{H}$ & 4.85553700 & 0.38547800 & 1.64583000 \\
\hline $\mathrm{H}$ & -4.54213300 & -0.89970600 & 2.28696900 \\
\hline $\mathrm{H}$ & -4.75631900 & 0.90396700 & 0.68211900 \\
\hline $\mathrm{H}$ & -6.21677100 & -1.00753400 & 0.57260400 \\
\hline $\mathrm{H}$ & -3.36158500 & -0.00353600 & -1.96599900 \\
\hline $\mathrm{H}$ & -1.77854500 & -1.87639600 & -3.04213700 \\
\hline $\mathrm{H}$ & -1.66479600 & -3.96088500 & -1.33081700 \\
\hline $\mathrm{H}$ & -3.17099900 & -3.35938900 & 0.80178700 \\
\hline $\mathrm{C}$ & 0.04374200 & 0.86730100 & 2.54191100 \\
\hline $\mathrm{C}$ & 1.24990500 & 0.18247300 & 2.53115200 \\
\hline $\mathrm{C}$ & 1.31263700 & -1.18911600 & 2.08138400 \\
\hline $\mathrm{C}$ & 0.06813400 & -1.93569800 & 2.14040200 \\
\hline $\mathrm{C}$ & -1.14819800 & -1.27603200 & 2.14600500 \\
\hline $\mathrm{C}$ & -1.12261900 & 0.19256100 & 1.92975300 \\
\hline $\mathrm{H}$ & -0.06466900 & 1.78064100 & 3.11601000 \\
\hline $\mathrm{H}$ & 2.14750900 & 0.64820200 & 2.92920000 \\
\hline $\mathrm{H}$ & 2.22591800 & -1.73756400 & 2.29326800 \\
\hline $\mathrm{H}$ & 0.10978300 & -3.01349200 & 2.27439400 \\
\hline
\end{tabular}




$\begin{array}{lrrr}\mathrm{H} & -2.05359500 & -1.78429500 & 2.46073600 \\ \mathrm{H} & -2.07571100 & 0.69625600 & 2.07779200\end{array}$

\section{TS8 $^{\mathrm{T}}$}

$\mathrm{Ti}$

Ti

Ti

$\mathrm{Si}$

$\mathrm{Si}$

$\mathrm{Si}$

C

C

C

C

C

C

C

C

C

C

C

C

C

C

C

$\mathrm{H}$

$\mathrm{H}$

$\mathrm{H}$

$\mathrm{H}$

$\mathrm{H}$

$\mathrm{H}$

$\mathrm{H}$

$\mathrm{H}$

$\mathrm{H}$

$\mathrm{H}$

$\mathrm{H}$

$\mathrm{H}$

$\mathrm{H}$

$\mathrm{H}$

$\mathrm{H}$

$\mathrm{H}$

$\mathrm{H}$

$\mathrm{H}$

$\mathrm{H}$

$\mathrm{H}$

$\mathrm{H}$

$\begin{array}{ccc}-1.27601400 & -1.10304800 & -0.09690300 \\ 1.59713700 & -0.83114900 & -0.18658100 \\ 0.20648800 & 1.36785600 & 0.36051500 \\ 5.09508000 & 0.40638900 & -0.01089800 \\ -4.91522500 & -0.53399100 & 0.77785000 \\ -2.22255000 & 3.06257800 & -1.98150500 \\ 3.92675000 & -0.89659300 & -0.65734900 \\ 3.22559000 & -0.87504200 & -1.90681300 \\ 2.49747500 & -2.07931600 & -2.04601100 \\ 2.73406000 & -2.87067500 & -0.88873100 \\ 3.61326400 & -2.15031000 & -0.04509200 \\ -3.66461500 & -1.43887500 & -0.26248000 \\ -3.19375900 & -1.05862000 & -1.55726800 \\ -2.31594500 & -2.05577000 & -2.04205800 \\ -2.23066700 & -3.08166000 & -1.05968400 \\ -3.05591400 & -2.70378500 & 0.02638100 \\ -0.78654600 & 3.21925200 & -0.79903600 \\ 0.59933000 & 3.18453200 & -1.13957700 \\ 1.36828300 & 3.44536600 & 0.02403200 \\ 0.47202100 & 3.63733700 & 1.10338900 \\ -0.84834100 & 3.48791900 & 0.60421600 \\ 0.26420200 & -2.14059200 & 0.26889500 \\ 0.18479600 & -1.21409900 & -1.25491300 \\ 1.98770700 & 0.88608600 & 0.37480100 \\ 0.93839400 & 0.60185500 & -1.09767100 \\ -1.11708100 & 0.69875200 & -0.62523100 \\ 2.44898900 & 3.46819200 & 0.07930100 \\ 0.99968500 & 2.98223500 & -2.12478000 \\ 0.74894400 & 3.85144700 & 2.12783200 \\ -1.75760100 & 3.55888300 & 1.19069400 \\ -3.42174600 & 2.53880000 & -1.27966900 \\ -2.57584900 & 4.40009900 & -2.53999900 \\ -1.86937700 & 2.17793500 & -3.12130600 \\ 1.85556000 & -2.34237300 & -2.87652100 \\ 3.23153100 & -0.05739600 & -2.61744700 \\ 2.31287200 & -3.84872800 & -0.69004600 \\ 3.99035800 & -2.49360100 & 0.91118200 \\ 6.50996700 & 0.07804800 & -0.34713100 \\ 4.77852700 & 1.72716700 & -0.61447100 \\ 5.00343800 & 0.50070300 & 1.47195300 \\ -4.56359400 & -0.62803200 & 2.22364100 \\ & 0.89923200 & 0.38518500\end{array}$

S98 


$\begin{array}{lrrr}\mathrm{H} & -6.28439000 & -1.10277100 & 0.62347300 \\ \mathrm{H} & -3.44599800 & -0.13972700 & -2.07181500 \\ \mathrm{H} & -1.78560700 & -2.03097500 & -2.98579400 \\ \mathrm{H} & -1.64088800 & -3.98737600 & -1.13235000 \\ \mathrm{H} & -3.20725700 & -3.28272700 & 0.93017800 \\ \mathrm{C} & 0.04201100 & 0.90782000 & 2.56488900 \\ \mathrm{C} & 1.26336700 & 0.24138100 & 2.57100100 \\ \mathrm{C} & 1.38615500 & -1.10547500 & 2.09832300 \\ \mathrm{C} & 0.15933600 & -1.88367300 & 1.90485800 \\ \mathrm{C} & -1.12273100 & -1.25675400 & 2.03212500 \\ \mathrm{C} & -1.10674500 & 0.23141500 & 1.95377400 \\ \mathrm{H} & -0.07178800 & 1.83235000 & 3.11986400 \\ \mathrm{H} & 2.14381900 & 0.72766200 & 2.98339300 \\ \mathrm{H} & 2.28922200 & -1.65015900 & 2.35532400 \\ \mathrm{H} & 0.22152900 & -2.95336900 & 2.09430600 \\ \mathrm{H} & -1.91760200 & -1.78880400 & 2.54702000 \\ \mathrm{H} & -2.06894500 & 0.71024800 & 2.12783300\end{array}$

\begin{tabular}{|c|c|c|c|}
\hline $\mathbf{A} 9^{\mathrm{T}}$ & & & \\
\hline $\mathrm{Ti}$ & -1.05526700 & -1.04173000 & -0.25372600 \\
\hline $\mathrm{Ti}$ & 1.84406300 & -0.78675800 & 0.17813300 \\
\hline $\mathrm{Ti}$ & -0.01337400 & 1.33856200 & 0.53941000 \\
\hline $\mathrm{Si}$ & 4.13131500 & 1.32566800 & -1.81349500 \\
\hline $\mathrm{Si}$ & -4.80419700 & -1.29075500 & 0.00742800 \\
\hline $\mathrm{Si}$ & -2.75514100 & 3.24197200 & -1.25652800 \\
\hline $\mathrm{C}$ & 3.79952500 & -0.34096200 & -1.04654600 \\
\hline $\mathrm{C}$ & 3.16534500 & -1.46060000 & -1.67151900 \\
\hline $\mathrm{C}$ & 3.19567500 & -2.55932300 & -0.77679200 \\
\hline $\mathrm{C}$ & 3.85420900 & -2.14454700 & 0.40820200 \\
\hline $\mathrm{C}$ & 4.21957800 & -0.78588800 & 0.25315500 \\
\hline $\mathrm{C}$ & -3.24042600 & -1.74677900 & -0.90656900 \\
\hline $\mathrm{C}$ & -2.67231200 & -1.04976400 & -2.02169800 \\
\hline $\mathrm{C}$ & -1.55501800 & -1.77219600 & -2.49143500 \\
\hline $\mathrm{C}$ & -1.42141200 & -2.94050800 & -1.68866700 \\
\hline $\mathrm{C}$ & -2.45226200 & -2.92887200 & -0.72176500 \\
\hline $\mathrm{C}$ & -1.19290400 & 3.29242800 & -0.23486600 \\
\hline $\mathrm{C}$ & 0.14520500 & 3.34727500 & -0.73150500 \\
\hline $\mathrm{C}$ & 1.03375500 & 3.49305400 & 0.36420300 \\
\hline $\mathrm{C}$ & 0.26226800 & 3.51184400 & 1.55427600 \\
\hline $\mathrm{C}$ & -1.10012600 & 3.38389200 & 1.18774700 \\
\hline $\mathrm{H}$ & 0.15286500 & -3.30009600 & 1.85055000 \\
\hline $\mathrm{H}$ & 0.47833600 & -1.98395400 & -0.22942700 \\
\hline $\mathrm{H}$ & 1.76795700 & 1.05128500 & 0.44562700 \\
\hline $\mathrm{H}$ & 0.41601800 & 0.05956700 & -0.78608100 \\
\hline $\mathrm{H}$ & -1.36489400 & 0.73160000 & -0.52597300 \\
\hline $\mathrm{H}$ & 2.11208100 & 3.55697600 & 0.30279400 \\
\hline
\end{tabular}




\begin{tabular}{|c|c|c|c|}
\hline $\mathrm{H}$ & 0.43424000 & 3.27707900 & -1.77349600 \\
\hline $\mathrm{H}$ & 0.64910400 & 3.59434700 & 2.56204500 \\
\hline $\mathrm{H}$ & -1.93784000 & 3.34408500 & 1.87543100 \\
\hline $\mathrm{H}$ & -3.84078500 & 2.54658400 & -0.51902000 \\
\hline $\mathrm{H}$ & -3.22257400 & 4.62594000 & -1.55457900 \\
\hline $\mathrm{H}$ & -2.50690500 & 2.56065300 & -2.55378900 \\
\hline $\mathrm{H}$ & 2.76652600 & -3.53762900 & -0.95593100 \\
\hline $\mathrm{H}$ & 2.71918200 & -1.46424800 & -2.65913500 \\
\hline $\mathrm{H}$ & 4.02613900 & -2.75601800 & 1.28551400 \\
\hline $\mathrm{H}$ & 4.73539600 & -0.18046000 & 0.98972000 \\
\hline $\mathrm{H}$ & 5.38216800 & 1.30564100 & -2.62452100 \\
\hline $\mathrm{H}$ & 3.01863000 & 1.72324100 & -2.71730100 \\
\hline $\mathrm{H}$ & 4.30067900 & 2.35564700 & -0.75380100 \\
\hline $\mathrm{H}$ & -4.68931800 & -1.63960600 & 1.44958100 \\
\hline $\mathrm{H}$ & -5.06521100 & 0.16568000 & -0.12669000 \\
\hline $\mathrm{H}$ & -5.97510900 & -2.02869900 & -0.54262300 \\
\hline $\mathrm{H}$ & -3.02176700 & -0.10502300 & -2.41812300 \\
\hline $\mathrm{H}$ & -0.89675100 & -1.47239500 & -3.29786000 \\
\hline $\mathrm{H}$ & -0.65265800 & -3.69554900 & -1.78901100 \\
\hline $\mathrm{H}$ & -2.61217300 & -3.68391400 & 0.03722200 \\
\hline $\mathrm{C}$ & -0.12446600 & 0.57371600 & 2.54941500 \\
\hline $\mathrm{C}$ & 1.12218500 & -0.14765300 & 2.67552000 \\
\hline $\mathrm{C}$ & 1.22785600 & -1.50639600 & 2.41058800 \\
\hline $\mathrm{C}$ & -0.03361100 & -2.36099800 & 2.38315300 \\
\hline $\mathrm{C}$ & -1.22691400 & -1.62017000 & 1.77240800 \\
\hline $\mathrm{C}$ & -1.23822900 & -0.15279300 & 1.93638000 \\
\hline $\mathrm{H}$ & -0.32292400 & 1.36953800 & 3.26288700 \\
\hline $\mathrm{H}$ & 1.99295700 & 0.39989200 & 3.03362700 \\
\hline $\mathrm{H}$ & 2.15396100 & -2.00093300 & 2.69754000 \\
\hline $\mathrm{H}$ & -0.26976700 & -2.64156000 & 3.42788100 \\
\hline $\mathrm{H}$ & -2.18295600 & -2.09467200 & 2.00142400 \\
\hline $\mathrm{H}$ & -2.21909100 & 0.31042400 & 2.05574100 \\
\hline \multicolumn{4}{|c|}{$3^{T}$} \\
\hline $\mathrm{Ti}$ & 0.00984700 & 1.36532700 & -0.57112300 \\
\hline $\mathrm{Ti}$ & 1.44185400 & -0.77993600 & 0.38556400 \\
\hline $\mathrm{Ti}$ & -1.31009400 & -0.98448300 & 0.24849800 \\
\hline $\mathrm{Si}$ & -0.48093000 & 3.34233300 & 2.53989000 \\
\hline $\mathrm{Si}$ & 5.02254900 & -0.56573200 & -0.93439000 \\
\hline $\mathrm{Si}$ & -4.91705700 & -0.80939900 & -0.90750600 \\
\hline $\mathrm{C}$ & 1.20857200 & -0.05050800 & -2.00736700 \\
\hline $\mathrm{C}$ & -0.03403500 & 0.49540900 & -2.57730400 \\
\hline $\mathrm{C}$ & -1.22066100 & -0.27668800 & -2.45684400 \\
\hline $\mathrm{C}$ & -1.24521100 & -1.56577100 & -1.86091200 \\
\hline $\mathrm{C}$ & 0.00412900 & -2.09405900 & -1.30412100 \\
\hline $\mathrm{C}$ & 1.22639100 & -1.58645500 & -2.08480000 \\
\hline
\end{tabular}




\begin{tabular}{|c|c|c|c|}
\hline $\mathrm{C}$ & -0.26688400 & 3.36743800 & 0.68739900 \\
\hline $\mathrm{C}$ & 0.96338500 & 3.48487900 & -0.03396000 \\
\hline $\mathrm{C}$ & 0.68660500 & 3.51451400 & -1.42153800 \\
\hline $\mathrm{C}$ & -0.72238200 & 3.42918200 & -1.58605900 \\
\hline $\mathrm{C}$ & -1.30391400 & 3.34389000 & -0.30002400 \\
\hline $\mathrm{C}$ & 3.85865600 & -1.05927900 & 0.43639600 \\
\hline $\mathrm{C}$ & 3.47433800 & -0.24351200 & 1.54997700 \\
\hline $\mathrm{C}$ & 2.66271600 & -1.00015500 & 2.42430200 \\
\hline $\mathrm{C}$ & 2.53427000 & -2.30533100 & 1.86834200 \\
\hline $\mathrm{C}$ & 3.27168700 & -2.34244100 & 0.65998400 \\
\hline $\mathrm{C}$ & -3.29181400 & -0.51701900 & 1.52925100 \\
\hline $\mathrm{C}$ & -2.42756500 & -1.27329400 & 2.34746400 \\
\hline $\mathrm{C}$ & -2.29591500 & -2.56558700 & 1.75899100 \\
\hline $\mathrm{C}$ & -3.07858900 & -2.59166500 & 0.58421400 \\
\hline $\mathrm{C}$ & -3.69876200 & -1.31376000 & 0.41033300 \\
\hline $\mathrm{H}$ & -0.08414500 & 0.24231000 & 0.90460400 \\
\hline $\mathrm{H}$ & 1.52917500 & 1.06706100 & 0.35861800 \\
\hline $\mathrm{H}$ & 0.04471400 & -1.60425100 & 1.26781200 \\
\hline $\mathrm{H}$ & -1.65583000 & 0.77254200 & -0.10734800 \\
\hline $\mathrm{H}$ & 2.11188000 & 0.43078600 & -2.38908900 \\
\hline $\mathrm{H}$ & -0.02198600 & 1.30405300 & -3.30325600 \\
\hline $\mathrm{H}$ & -2.13895800 & 0.11021600 & -2.89449500 \\
\hline $\mathrm{H}$ & -2.10551800 & -2.19992100 & -2.05554900 \\
\hline $\mathrm{H}$ & -0.00574900 & -3.17161500 & -1.12496100 \\
\hline $\mathrm{H}$ & 2.16065500 & -2.00531100 & -1.69852400 \\
\hline $\mathrm{H}$ & -6.29137300 & -1.28828700 & -0.58983300 \\
\hline $\mathrm{H}$ & -4.95862400 & 0.67341400 & -1.01318400 \\
\hline $\mathrm{H}$ & -4.54028900 & -1.38580800 & -2.22777500 \\
\hline $\mathrm{H}$ & -3.57267800 & 0.51519500 & 1.69832900 \\
\hline $\mathrm{H}$ & -1.93056700 & -0.92911200 & 3.24541400 \\
\hline $\mathrm{H}$ & -1.68775200 & -3.37687100 & 2.13849100 \\
\hline $\mathrm{H}$ & -3.17014900 & -3.43598100 & -0.08963100 \\
\hline $\mathrm{H}$ & -2.36375500 & 3.25430700 & -0.09614800 \\
\hline $\mathrm{H}$ & -1.25703400 & 3.41553200 & -2.52764500 \\
\hline $\mathrm{H}$ & 1.42143500 & 3.56990200 & -2.21624700 \\
\hline $\mathrm{H}$ & 1.94998600 & 3.52585000 & 0.40965500 \\
\hline $\mathrm{H}$ & -1.70784500 & 2.58489100 & 2.90519600 \\
\hline $\mathrm{H}$ & 0.70274300 & 2.72278300 & 3.19118700 \\
\hline $\mathrm{H}$ & -0.62750800 & 4.72227200 & 3.08531500 \\
\hline $\mathrm{H}$ & 6.42944600 & -0.93002800 & -0.60727100 \\
\hline $\mathrm{H}$ & 4.66334800 & -1.25624700 & -2.20288600 \\
\hline $\mathrm{H}$ & 4.96813500 & 0.90603400 & -1.14341200 \\
\hline $\mathrm{H}$ & 3.36456800 & -3.20359500 & 0.00785200 \\
\hline $\mathrm{H}$ & 1.96456700 & -3.12342000 & 2.29071600 \\
\hline $\mathrm{H}$ & 2.20341400 & -0.64600200 & 3.33870100 \\
\hline $\mathrm{H}$ & 3.74427100 & 0.79631000 & 1.68646400 \\
\hline
\end{tabular}




\begin{tabular}{|c|c|c|c|}
\hline $\mathrm{H}$ & 1.17212100 & -1.91650800 & -3.14001400 \\
\hline \multicolumn{4}{|c|}{ TS9 $^{T}$} \\
\hline $\mathrm{Ti}$ & 0.12846500 & 1.26596900 & -0.53243600 \\
\hline $\mathrm{Ti}$ & 1.45786200 & -1.01911800 & 0.18695100 \\
\hline $\mathrm{Ti}$ & -1.48310200 & -0.91840300 & 0.17617300 \\
\hline $\mathrm{Si}$ & -0.30090700 & 3.28970800 & 2.56385900 \\
\hline $\mathrm{Si}$ & 5.03631600 & 0.07161100 & -0.44405900 \\
\hline $\mathrm{Si}$ & -5.07184900 & -0.10188500 & -0.62236200 \\
\hline $\mathrm{C}$ & 1.30988800 & -0.13279200 & -1.75324500 \\
\hline $\mathrm{C}$ & 0.34438100 & 0.44683400 & -2.60148400 \\
\hline $\mathrm{C}$ & -1.03624800 & 0.10843300 & -2.43562300 \\
\hline $\mathrm{C}$ & -1.50218400 & -1.22145100 & -2.13013600 \\
\hline $\mathrm{C}$ & -0.70446600 & -2.27692000 & -1.69644800 \\
\hline $\mathrm{C}$ & 0.77624500 & -2.27689300 & -1.73939700 \\
\hline $\mathrm{C}$ & -0.00048300 & 3.28218600 & 0.72307100 \\
\hline $\mathrm{C}$ & 1.26853800 & 3.26222400 & 0.05879200 \\
\hline $\mathrm{C}$ & 1.06071600 & 3.31469200 & -1.33860000 \\
\hline $\mathrm{C}$ & -0.34275500 & 3.38563600 & -1.56568900 \\
\hline $\mathrm{C}$ & -0.98777100 & 3.37790300 & -0.30908400 \\
\hline $\mathrm{C}$ & 3.86226500 & -1.04263400 & 0.47899900 \\
\hline $\mathrm{C}$ & 3.24951400 & -0.76575200 & 1.74892200 \\
\hline $\mathrm{C}$ & 2.51935700 & -1.89986300 & 2.16845300 \\
\hline $\mathrm{C}$ & 2.65165500 & -2.89311300 & 1.16012400 \\
\hline $\mathrm{C}$ & 3.48101700 & -2.37276300 & 0.13682900 \\
\hline $\mathrm{C}$ & -3.23488400 & -0.37993100 & 1.66530200 \\
\hline $\mathrm{C}$ & -2.44981400 & -1.36626300 & 2.30795700 \\
\hline $\mathrm{C}$ & -2.56610800 & -2.56591400 & 1.55601800 \\
\hline $\mathrm{C}$ & -3.42488200 & -2.31424200 & 0.45965100 \\
\hline $\mathrm{C}$ & -3.85434200 & -0.95145900 & 0.50422700 \\
\hline $\mathrm{H}$ & -0.03268000 & 0.06731700 & 0.88068900 \\
\hline $\mathrm{H}$ & 1.60655600 & 0.82703100 & 0.39847600 \\
\hline $\mathrm{H}$ & -0.06089800 & -1.91956200 & 0.74827300 \\
\hline $\mathrm{H}$ & -1.56935400 & 0.91363500 & -0.02717000 \\
\hline $\mathrm{H}$ & 2.34591300 & -0.04629600 & -2.09365800 \\
\hline $\mathrm{H}$ & 0.63394400 & 1.10508500 & -3.42090300 \\
\hline $\mathrm{H}$ & -1.77691900 & 0.77499800 & -2.87391400 \\
\hline $\mathrm{H}$ & -2.56905200 & -1.39599200 & -2.25267000 \\
\hline $\mathrm{H}$ & -1.19770200 & -3.22083700 & -1.46367500 \\
\hline $\mathrm{H}$ & 1.19357300 & -3.16953400 & -1.26424800 \\
\hline $\mathrm{H}$ & -6.47855900 & -0.39729000 & -0.23061300 \\
\hline $\mathrm{H}$ & -4.87572800 & 1.37059400 & -0.56054200 \\
\hline $\mathrm{H}$ & -4.89735000 & -0.56239000 & -2.02814400 \\
\hline $\mathrm{H}$ & -3.33451900 & 0.64961100 & 1.98639600 \\
\hline $\mathrm{H}$ & -1.84197500 & -1.22460600 & 3.19278900 \\
\hline $\mathrm{H}$ & -2.06028500 & -3.49964400 & 1.76774700 \\
\hline
\end{tabular}




$\begin{array}{lrrr}\mathrm{H} & -3.69343100 & -3.03594700 & -0.30403200 \\ \mathrm{H} & -2.05892200 & 3.40485000 & -0.15343600 \\ \mathrm{H} & -0.83162200 & 3.41958900 & -2.53158300 \\ \mathrm{H} & 1.83063300 & 3.28697100 & -2.09932200 \\ \mathrm{H} & 2.23250300 & 3.19752300 & 0.54671100 \\ \mathrm{H} & -1.62716200 & 2.69086200 & 2.87171100 \\ \mathrm{H} & 0.76566500 & 2.52947000 & 3.26715800 \\ \mathrm{H} & -0.29857600 & 4.67871800 & 3.10556500 \\ \mathrm{H} & 6.45585800 & -0.22861500 & -0.10644700 \\ \mathrm{H} & 4.88106600 & -0.10344800 & -1.91484600 \\ \mathrm{H} & 4.77431700 & 1.49304200 & -0.09246400 \\ \mathrm{H} & 3.75810900 & -2.89241500 & -0.77318700 \\ \mathrm{H} & 2.18278700 & -3.86996000 & 1.16631500 \\ \mathrm{H} & 1.93383800 & -1.98568600 & 3.07544500 \\ \mathrm{H} & 3.33020200 & 0.16758200 & 2.29285900 \\ \mathrm{H} & 1.20116800 & -2.19006400 & -2.74037800\end{array}$

\section{B1 $^{\mathrm{T}}$}

$\begin{array}{ccc}-0.19205300 & 1.36936800 & -0.50210200 \\ 1.60394700 & -0.62068500 & 0.29412000 \\ -1.23024500 & -1.18686200 & 0.19201000 \\ -1.62329800 & 2.91781800 & 2.57311100 \\ 5.22947000 & -0.24097000 & -0.77533000 \\ -4.76766100 & -0.89101900 & -1.03046800 \\ 1.74990100 & 0.67137900 & -1.37320800 \\ 0.93032500 & 0.88388600 & -2.48628700 \\ -0.31701700 & 0.23515500 & -2.65788500 \\ -0.66028700 & -1.10369300 & -2.22111200 \\ 0.16296600 & -2.09094600 & -1.67093500 \\ 1.60737900 & -2.05775800 & -1.43291700 \\ -1.08516400 & 3.14013600 & 0.80016100 \\ 0.21296200 & 3.53999600 & 0.34886200 \\ 0.19424400 & 3.65308100 & -1.06194900 \\ -1.11886300 & 3.33366100 & -1.50884400 \\ -1.90066400 & 3.03000800 & -0.37059500 \\ 4.01007100 & -0.62478500 & 0.58480100 \\ 3.44892200 & 0.29010900 & 1.52550700 \\ 2.64035300 & -0.42546800 & 2.44382400 \\ 2.70315100 & -1.79986800 & 2.09726400 \\ 3.52711500 & -1.92022900 & 0.95386100 \\ -3.21695400 & -1.26848600 & 1.45309900 \\ -2.32368400 & -2.19989200 & 2.03288100 \\ -2.10638500 & -3.23985400 & 1.08382800 \\ -2.87248900 & -2.94688900 & -0.06843900 \\ -3.56715800 & -1.71544900 & 0.13438900 \\ -0.14351300 & 0.15120000 & 0.91264400\end{array}$




$\begin{array}{crrr}\mathrm{H} & 1.19897700 & 1.24769900 & 0.59742200 \\ \mathrm{H} & 0.35994500 & -1.82508300 & 0.88262100 \\ \mathrm{H} & -1.75954400 & 0.49350900 & -0.34652000 \\ \mathrm{H} & 2.61716300 & 1.33446800 & -1.31900700 \\ \mathrm{H} & 1.12958100 & 1.72097000 & -3.15961600 \\ \mathrm{H} & -1.01398200 & 0.67686600 & -3.36738400 \\ \mathrm{H} & -1.67578400 & -1.40728600 & -2.47349900 \\ \mathrm{H} & -0.33264500 & -3.04274900 & -1.46950200 \\ \mathrm{H} & 1.96229200 & -3.03132300 & -1.08958400 \\ \mathrm{H} & -6.11863200 & -1.51457800 & -0.94916200 \\ \mathrm{H} & -4.90586200 & 0.54806000 & -0.68484800 \\ \mathrm{H} & -4.30399400 & -1.01840300 & -2.43985800 \\ \mathrm{H} & -3.56114100 & -0.35191600 & 1.91711200 \\ \mathrm{H} & -1.86826700 & -2.12657200 & 3.01278300 \\ \mathrm{H} & -1.45203200 & -4.09325800 & 1.21505600 \\ \mathrm{H} & -2.90659400 & -3.54821500 & -0.97056100 \\ \mathrm{H} & -2.94022500 & 2.72835700 & -0.38495700 \\ \mathrm{H} & -1.45594900 & 3.31234300 & -2.53747900 \\ \mathrm{H} & 1.03590100 & 3.91863000 & -1.68882000 \\ \mathrm{H} & 1.07238600 & 3.71966500 & 0.98198600 \\ \mathrm{H} & -2.78128400 & 1.98911300 & 2.64954800 \\ \mathrm{H} & -0.50270400 & 2.39072700 & 3.39578100 \\ \mathrm{H} & -2.04690700 & 4.21807300 & 3.16644400 \\ \mathrm{H} & 6.62454400 & -0.48824800 & -0.31296700 \\ \mathrm{H} & 4.98538900 & -1.09725700 & -1.96469400 \\ \mathrm{H} & 5.12842400 & 1.19319200 & -1.15639100 \\ \mathrm{H} & 3.76004600 & -2.84465700 & 0.44173800 \\ \mathrm{H} & 2.18181000 & -2.60679600 & 2.59565800 \\ \mathrm{H} & 2.05614300 & 0.00701100 & 3.24705000 \\ \mathrm{H} & 3.59318800 & 1.36360400 & 1.52260200 \\ \mathrm{H} & 2.21549400 & -1.68339400 & -2.25243300\end{array}$

$\begin{array}{cccc}\text { B2 }^{\mathbf{T}} & & & \\ \mathrm{Ti} & -0.49272600 & 1.52575600 & -0.59697800 \\ \mathrm{Ti} & 1.76376000 & 0.03379200 & 0.45923100 \\ \mathrm{Ti} & -0.63139100 & -1.32185500 & 0.04917900 \\ \mathrm{Si} & -2.32200000 & 2.31420500 & 2.61374500 \\ \mathrm{Si} & 5.02260100 & -0.83964400 & -1.13206200 \\ \mathrm{Si} & -4.29780300 & -2.20240200 & -0.53879700 \\ \mathrm{C} & 1.52175500 & 1.06109700 & -1.37459300 \\ \mathrm{C} & 0.78780000 & 1.37933100 & -2.52365600 \\ \mathrm{C} & -0.54679500 & 0.96861300 & -2.73615100 \\ \mathrm{C} & -1.25181500 & -0.17962700 & -2.13984000 \\ \mathrm{C} & -0.84647900 & -1.56878400 & -2.29589000 \\ \mathrm{C} & 0.42065200 & -2.07877500 & -2.28808600 \\ \mathrm{C} & -1.80958600 & 2.86590000 & 0.90483700\end{array}$




\begin{tabular}{|c|c|c|c|}
\hline $\mathrm{C}$ & -0.64494400 & 3.61636500 & 0.56852200 \\
\hline $\mathrm{C}$ & -0.65389300 & 3.88685900 & -0.82092600 \\
\hline $\mathrm{C}$ & -1.84506200 & 3.32730100 & -1.36598700 \\
\hline $\mathrm{C}$ & -2.54655000 & 2.69739400 & -0.31222000 \\
\hline $\mathrm{C}$ & 4.10474000 & -0.43212400 & 0.43597700 \\
\hline $\mathrm{C}$ & 3.91149200 & 0.86425800 & 1.01521400 \\
\hline $\mathrm{C}$ & 3.22080900 & 0.73111500 & 2.24788800 \\
\hline $\mathrm{C}$ & 2.98110900 & -0.65019600 & 2.45657600 \\
\hline $\mathrm{C}$ & 3.51206300 & -1.35889100 & 1.35197700 \\
\hline $\mathrm{C}$ & -2.33400000 & -1.90161900 & 1.62351000 \\
\hline $\mathrm{C}$ & -1.13224300 & -2.48863400 & 2.08268300 \\
\hline $\mathrm{C}$ & -0.73281300 & -3.46937800 & 1.13341000 \\
\hline $\mathrm{C}$ & -1.70159800 & -3.48943900 & 0.10364800 \\
\hline $\mathrm{C}$ & -2.70890200 & -2.51335200 & 0.38414700 \\
\hline $\mathrm{H}$ & 0.33206400 & -0.64175800 & 1.39010400 \\
\hline $\mathrm{H}$ & 0.69786100 & 1.51070800 & 0.78096700 \\
\hline $\mathrm{H}$ & 1.14761300 & -1.65081200 & -0.07742000 \\
\hline $\mathrm{H}$ & -1.30573000 & 0.27158200 & 0.46846300 \\
\hline $\mathrm{H}$ & 2.44280300 & 1.65622200 & -1.28299300 \\
\hline $\mathrm{H}$ & 1.12455400 & 2.19196600 & -3.17245200 \\
\hline $\mathrm{H}$ & -1.10775600 & 1.52404300 & -3.48431100 \\
\hline $\mathrm{H}$ & -2.33131500 & -0.07319700 & -2.25057700 \\
\hline $\mathrm{H}$ & -1.66928400 & -2.27785300 & -2.35666800 \\
\hline $\mathrm{H}$ & 0.57523900 & -3.15055000 & -2.36328600 \\
\hline $\mathrm{H}$ & -5.34747700 & -3.19264300 & -0.17132000 \\
\hline $\mathrm{H}$ & -4.80956200 & -0.84357300 & -0.21861400 \\
\hline $\mathrm{H}$ & -4.08675900 & -2.30804900 & -2.01026800 \\
\hline $\mathrm{H}$ & -2.88023600 & -1.11274700 & 2.12434000 \\
\hline $\mathrm{H}$ & -0.60378500 & -2.22789500 & 2.98952900 \\
\hline $\mathrm{H}$ & 0.15840100 & -4.08201900 & 1.18414100 \\
\hline $\mathrm{H}$ & -1.67190200 & -4.13534900 & -0.76643000 \\
\hline $\mathrm{H}$ & -3.47827700 & 2.15267700 & -0.41009300 \\
\hline $\mathrm{H}$ & -2.15776700 & 3.38119700 & -2.39962000 \\
\hline $\mathrm{H}$ & 0.11128000 & 4.41988000 & -1.37174100 \\
\hline $\mathrm{H}$ & 0.13952400 & 3.90183400 & 1.25746700 \\
\hline $\mathrm{H}$ & -3.34858600 & 1.24500600 & 2.51228900 \\
\hline $\mathrm{H}$ & -1.15892600 & 1.82895200 & 3.39834400 \\
\hline $\mathrm{H}$ & -2.93108400 & 3.45261300 & 3.35940500 \\
\hline $\mathrm{H}$ & 6.46189300 & -1.12968300 & -0.86841800 \\
\hline $\mathrm{H}$ & 4.43714400 & -2.04360300 & -1.78094000 \\
\hline $\mathrm{H}$ & 4.96505500 & 0.31160700 & -2.07293100 \\
\hline $\mathrm{H}$ & 3.45755500 & -2.43161300 & 1.21053100 \\
\hline $\mathrm{H}$ & 2.44588700 & -1.08306300 & 3.29267900 \\
\hline $\mathrm{H}$ & 2.92129400 & 1.53885200 & 2.90390300 \\
\hline $\mathrm{H}$ & 4.22643600 & 1.80332300 & 0.57302100 \\
\hline $\mathrm{H}$ & 1.29808200 & -1.45001100 & -2.34526300 \\
\hline
\end{tabular}




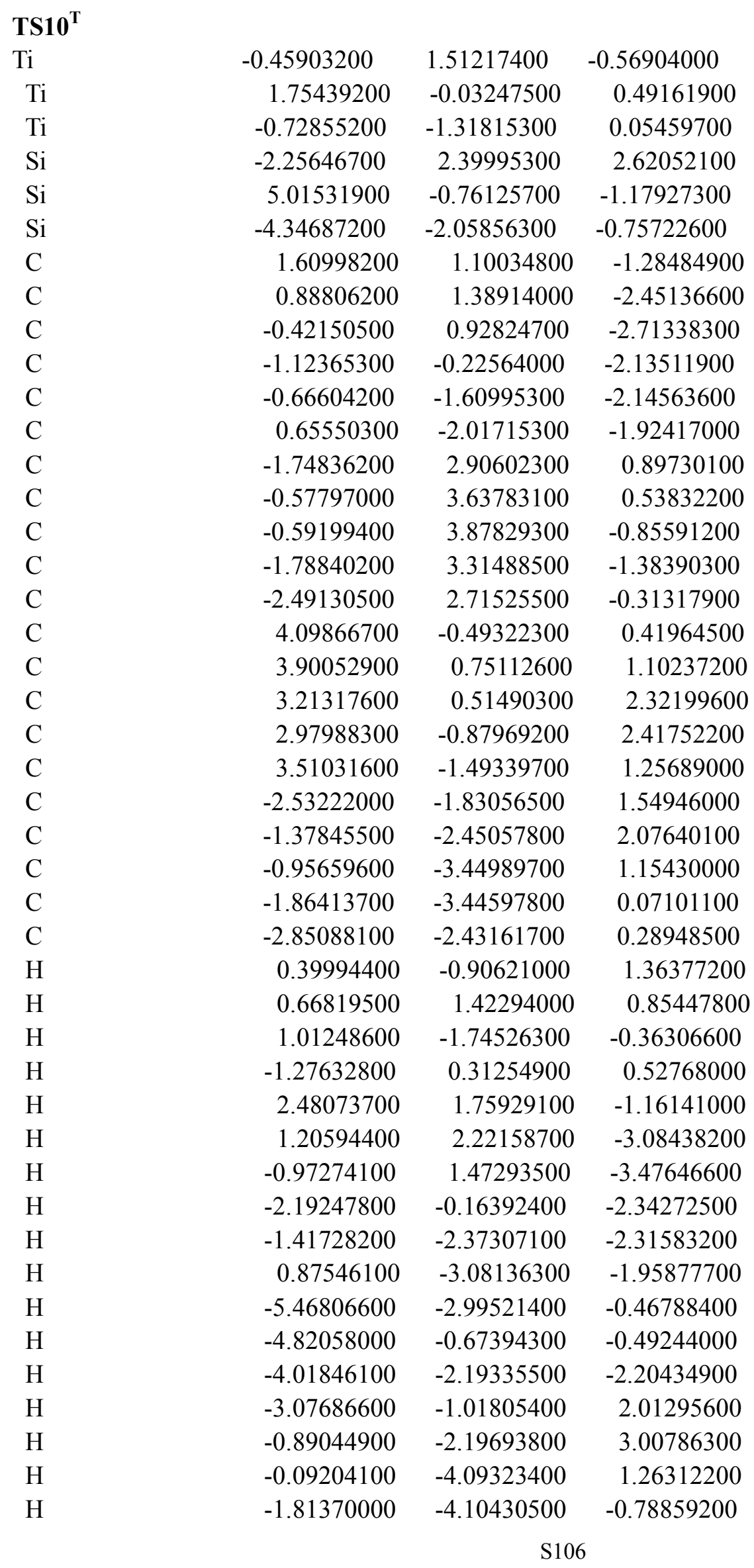




$\begin{array}{lrrr}\mathrm{H} & -3.42713800 & 2.17505700 & -0.39572500 \\ \mathrm{H} & -2.10263900 & 3.34299300 & -2.41835800 \\ \mathrm{H} & 0.17616400 & 4.39108600 & -1.42118800 \\ \mathrm{H} & 0.21388800 & 3.92849700 & 1.21701300 \\ \mathrm{H} & -3.28538300 & 1.33012300 & 2.55343900 \\ \mathrm{H} & -1.09169900 & 1.93446500 & 3.41507800 \\ \mathrm{H} & -2.86219100 & 3.55712200 & 3.34037800 \\ \mathrm{H} & 6.42432800 & -1.18684900 & -0.93924700 \\ \mathrm{H} & 4.36358700 & -1.82853400 & -1.98732800 \\ \mathrm{H} & 5.05216200 & 0.50188200 & -1.96295800 \\ \mathrm{H} & 3.45986700 & -2.55236700 & 1.03000000 \\ \mathrm{H} & 2.44757300 & -1.38218100 & 3.21553200 \\ \mathrm{H} & 2.91229300 & 1.26494600 & 3.04269100 \\ \mathrm{H} & 4.20903700 & 1.72393500 & 0.73558500 \\ \mathrm{H} & 1.48130400 & -1.37061900 & -2.19616900\end{array}$

B3 $^{\mathrm{T}}$

Ti

Ti

$\mathrm{Ti}$

$\mathrm{Si}$

$\mathrm{Si}$

$\mathrm{Si}$

C

C

C

C

C

C

C

C

C

C

C

C

C

C

C

C

C

C

C

C

C

$\mathrm{H}$

$\mathrm{H}$

$\begin{array}{ccc}-0.28484200 & 1.48544400 & -0.73554400 \\ 1.69889800 & 0.04227600 & 0.42435000 \\ -0.78058100 & -1.54482000 & -0.17981000 \\ -1.43204900 & 2.60986400 & 2.67338200 \\ 4.98136000 & -1.17228000 & -0.89397500 \\ -4.49165300 & -1.28618800 & 0.40550700 \\ 1.58478100 & 0.82583200 & -1.56911900 \\ 0.82724200 & 1.08216700 & -2.72751800 \\ -0.55079200 & 0.81156400 & -2.83552900 \\ -1.33733100 & -0.12257800 & -2.00336200 \\ -1.26764400 & -1.61843500 & -2.17113000 \\ 0.04170800 & -2.23397800 & -2.61629800 \\ -1.39784600 & 2.90977900 & 0.83198600 \\ -0.44622800 & 3.72172500 & 0.13575100 \\ -0.80561100 & 3.79086900 & -1.22545400 \\ -1.99886100 & 3.03323200 & -1.39945500 \\ -2.36466700 & 2.49972600 & -0.14181800 \\ 4.01415300 & -0.53277400 & 0.56676700 \\ 3.87491100 & 0.82923700 & 0.97920400 \\ 3.12703200 & 0.88145200 & 2.18394800 \\ 2.78795900 & -0.44633900 & 2.53952200 \\ 3.32286400 & -1.31106000 & 1.55138400 \\ -2.01257200 & -1.90615700 & 1.87027800 \\ -0.96451800 & -2.85275300 & 1.85118900 \\ -1.17386800 & -3.72099200 & 0.74449200 \\ -2.35670300 & -3.30291200 & 0.08800900 \\ -2.89288300 & -2.16181000 & 0.77382200 \\ 1.03847000 & -1.63583100 & 0.26054000 \\ 1.00078400 & 1.70313200 & 0.56452300 \\ & & \end{array}$




\begin{tabular}{|c|c|c|c|}
\hline $\mathrm{H}$ & 0.81933100 & -2.20105300 & -1.82207100 \\
\hline $\mathrm{H}$ & -0.24646400 & 0.15033100 & 0.59749200 \\
\hline $\mathrm{H}$ & 2.60364800 & 1.22536000 & -1.62239700 \\
\hline $\mathrm{H}$ & 1.24285700 & 1.73417800 & -3.50003600 \\
\hline $\mathrm{H}$ & -1.08079300 & 1.35178700 & -3.61880400 \\
\hline $\mathrm{H}$ & -2.38903100 & 0.17412500 & -2.01331500 \\
\hline $\mathrm{H}$ & -2.16034400 & -2.05870800 & -2.61371900 \\
\hline $\mathrm{H}$ & -0.09561100 & -3.28473400 & -2.88685600 \\
\hline $\mathrm{H}$ & -5.67572400 & -2.06413000 & 0.86778200 \\
\hline $\mathrm{H}$ & -4.51552400 & 0.03011900 & 1.09977100 \\
\hline $\mathrm{H}$ & -4.65958800 & -1.05764100 & -1.05736400 \\
\hline $\mathrm{H}$ & -2.11450700 & -1.09342800 & 2.57967300 \\
\hline $\mathrm{H}$ & -0.13002900 & -2.89097200 & 2.54027900 \\
\hline $\mathrm{H}$ & -0.54088400 & -4.55204700 & 0.45637700 \\
\hline $\mathrm{H}$ & -2.79246100 & -3.77643900 & -0.78280300 \\
\hline $\mathrm{H}$ & -3.22485100 & 1.87082900 & 0.05000200 \\
\hline $\mathrm{H}$ & -2.53116900 & 2.89133500 & -2.33053300 \\
\hline $\mathrm{H}$ & -0.25483900 & 4.30153600 & -2.00649600 \\
\hline $\mathrm{H}$ & 0.43384500 & 4.17258400 & 0.57717300 \\
\hline $\mathrm{H}$ & -2.03326600 & 1.29006300 & 2.99222900 \\
\hline $\mathrm{H}$ & -0.05402600 & 2.67861600 & 3.22617700 \\
\hline $\mathrm{H}$ & -2.24843800 & 3.65201100 & 3.35823300 \\
\hline $\mathrm{H}$ & 6.38221300 & -1.51005300 & -0.51323300 \\
\hline $\mathrm{H}$ & 4.34964500 & -2.40343600 & -1.43809300 \\
\hline $\mathrm{H}$ & 5.04068900 & -0.13000100 & -1.95413700 \\
\hline $\mathrm{H}$ & 3.22014200 & -2.38913800 & 1.53894300 \\
\hline $\mathrm{H}$ & 2.19530800 & -0.74457400 & 3.39641700 \\
\hline $\mathrm{H}$ & 2.84681300 & 1.77870700 & 2.72116800 \\
\hline $\mathrm{H}$ & 4.25891000 & 1.69170000 & 0.44555200 \\
\hline $\mathrm{H}$ & 0.50447900 & -1.70232400 & -3.45922300 \\
\hline \multicolumn{4}{|c|}{$1^{\mathrm{AF}}$} \\
\hline $\mathrm{Ti}$ & 0.36578700 & 1.32084800 & -0.42739300 \\
\hline $\mathrm{Ti}$ & -1.39518200 & -0.69634400 & -0.55477700 \\
\hline $\mathrm{Ti}$ & 1.01533900 & -1.09454000 & 0.56050800 \\
\hline $\mathrm{Si}$ & -4.39008400 & -0.81425800 & 1.64098300 \\
\hline $\mathrm{Si}$ & -0.03893600 & 4.14800100 & 1.95537800 \\
\hline $\mathrm{Si}$ & 4.27564000 & -1.51413700 & -1.17862000 \\
\hline $\mathrm{C}$ & -3.65605400 & -1.15487500 & -0.03993400 \\
\hline $\mathrm{C}$ & -3.04910000 & -2.37332600 & -0.48961000 \\
\hline $\mathrm{C}$ & -2.68165800 & -2.23923000 & -1.85291000 \\
\hline $\mathrm{C}$ & -3.04716900 & -0.93586000 & -2.27325800 \\
\hline $\mathrm{C}$ & -3.64083800 & -0.27314300 & -1.16787000 \\
\hline $\mathrm{C}$ & 0.48847800 & 3.57939700 & 0.25857700 \\
\hline $\mathrm{C}$ & 1.80023600 & 3.16733700 & -0.14776900 \\
\hline $\mathrm{C}$ & 1.79354500 & 2.88382000 & -1.53736600 \\
\hline
\end{tabular}




\begin{tabular}{|c|c|c|c|}
\hline $\mathrm{C}$ & 0.47889100 & 3.10747400 & -2.01800500 \\
\hline $\mathrm{C}$ & -0.31802700 & 3.53167700 & -0.92325000 \\
\hline $\mathrm{C}$ & 3.14853200 & -2.03598200 & 0.22291200 \\
\hline $\mathrm{C}$ & 2.18932400 & -3.09997200 & 0.20552200 \\
\hline $\mathrm{C}$ & 1.58994800 & -3.20286400 & 1.48649600 \\
\hline $\mathrm{C}$ & 2.16681500 & -2.20453400 & 2.31562900 \\
\hline $\mathrm{C}$ & 3.12150100 & -1.49313500 & 1.54726600 \\
\hline $\mathrm{H}$ & 1.44524900 & 0.60198500 & 1.02478200 \\
\hline $\mathrm{H}$ & 1.55684000 & -0.09254000 & -0.81317500 \\
\hline $\mathrm{H}$ & -1.48320400 & 1.14148100 & -0.25010700 \\
\hline $\mathrm{H}$ & -0.46707800 & 0.35055700 & -1.76613900 \\
\hline $\mathrm{H}$ & -0.63367000 & -1.76369900 & 0.88478100 \\
\hline $\mathrm{H}$ & 0.18146600 & -1.67886700 & -0.90762600 \\
\hline $\mathrm{H}$ & -0.37256600 & 0.10755200 & 0.79191500 \\
\hline $\mathrm{H}$ & -5.82453700 & -1.21767900 & 1.69745900 \\
\hline $\mathrm{H}$ & -3.66325400 & -1.58871700 & 2.68213100 \\
\hline $\mathrm{H}$ & -4.31203600 & 0.63771500 & 1.94838600 \\
\hline $\mathrm{H}$ & -2.87659500 & -3.25101000 & 0.12206600 \\
\hline $\mathrm{H}$ & -2.17745600 & -2.98571900 & -2.45437400 \\
\hline $\mathrm{H}$ & -2.87117500 & -0.50853400 & -3.25241500 \\
\hline $\mathrm{H}$ & -4.00277100 & 0.74767700 & -1.17126200 \\
\hline $\mathrm{H}$ & 1.94545400 & -3.71360600 & -0.65241400 \\
\hline $\mathrm{H}$ & 0.81237200 & -3.89884000 & 1.77271100 \\
\hline $\mathrm{H}$ & 1.89376200 & -1.99521700 & 3.34400000 \\
\hline $\mathrm{H}$ & 3.71403600 & -0.65735700 & 1.89725000 \\
\hline $\mathrm{H}$ & 4.65527800 & -0.08754300 & -1.01718800 \\
\hline $\mathrm{H}$ & 5.52423800 & -2.32783900 & -1.15743000 \\
\hline $\mathrm{H}$ & 3.60749800 & -1.72981700 & -2.48612400 \\
\hline $\mathrm{H}$ & 2.63323200 & 2.52359000 & -2.11900800 \\
\hline $\mathrm{H}$ & 0.13428600 & 2.94821600 & -3.03202300 \\
\hline $\mathrm{H}$ & -1.37585500 & 3.75956400 & -0.97028300 \\
\hline $\mathrm{H}$ & 2.65722500 & 3.06610800 & 0.50760300 \\
\hline $\mathrm{H}$ & -1.48601200 & 3.87635300 & 2.15861100 \\
\hline $\mathrm{H}$ & 0.75581500 & 3.44876100 & 3.00016200 \\
\hline $\mathrm{H}$ & 0.17949300 & 5.61304500 & 2.12731900 \\
\hline
\end{tabular}

$\begin{array}{lccc}\mathbf{A 1}^{\mathbf{A F}} & & & \\ \mathrm{Ti} & 1.17154300 & -1.58994700 & -0.62895400 \\ \mathrm{Ti} & 0.04027700 & 0.13245400 & 1.09594800 \\ \mathrm{Si} & -4.81318000 & 0.08060100 & 0.33906400 \\ \mathrm{Si} & 4.38126500 & 0.25819000 & -1.24023800 \\ \mathrm{Si} & 2.01467600 & -0.31505500 & 4.22481400 \\ \mathrm{C} & -3.86867800 & -1.41994700 & -0.25882000 \\ \mathrm{C} & -3.39609400 & -2.51401300 & 0.53480200 \\ \mathrm{C} & -2.78104600 & -3.46952800 & -0.31500200 \\ \mathrm{C} & -2.86353500 & -2.98506600 & -1.64788900\end{array}$




\begin{tabular}{|c|c|c|c|}
\hline $\mathrm{C}$ & -3.52495600 & -1.73284400 & -1.61193000 \\
\hline $\mathrm{C}$ & 3.44135200 & -1.35279900 & -1.34505200 \\
\hline $\mathrm{C}$ & 3.39861200 & -2.39046900 & -0.35642600 \\
\hline $\mathrm{C}$ & 2.62584800 & -3.46686700 & -0.85095800 \\
\hline $\mathrm{C}$ & 2.16769300 & -3.10915700 & -2.14808400 \\
\hline $\mathrm{C}$ & 2.67243800 & -1.81685400 & -2.45443300 \\
\hline $\mathrm{C}$ & 0.94002000 & 0.80868800 & 3.19038900 \\
\hline $\mathrm{C}$ & -0.47931300 & 0.94187300 & 3.26790300 \\
\hline $\mathrm{C}$ & -0.90062400 & 1.92925200 & 2.33972300 \\
\hline $\mathrm{C}$ & 0.24797200 & 2.42963600 & 1.67024000 \\
\hline $\mathrm{C}$ & 1.37359700 & 1.74055700 & 2.19056500 \\
\hline $\mathrm{H}$ & -0.38267500 & -1.82274400 & -1.78344200 \\
\hline $\mathrm{H}$ & -0.24826700 & -2.65933400 & 0.00248900 \\
\hline $\mathrm{H}$ & -1.15095400 & -1.32104700 & 1.32943500 \\
\hline $\mathrm{H}$ & 1.15881400 & -1.33646700 & 1.20430000 \\
\hline $\mathrm{H}$ & 1.50980800 & 0.15357800 & -0.06530000 \\
\hline $\mathrm{H}$ & -1.92522600 & 2.22572100 & 2.15191000 \\
\hline $\mathrm{H}$ & -1.13248200 & 0.36028500 & 3.90813800 \\
\hline $\mathrm{H}$ & 0.25866500 & 3.17648300 & 0.88622000 \\
\hline $\mathrm{H}$ & 2.39770800 & 1.87584600 & 1.86453600 \\
\hline $\mathrm{H}$ & 3.26948400 & -0.64457300 & 3.50028100 \\
\hline $\mathrm{H}$ & 2.39415600 & 0.34956000 & 5.50524400 \\
\hline $\mathrm{H}$ & 1.28160800 & -1.56241100 & 4.56580200 \\
\hline $\mathrm{H}$ & -2.31001100 & -4.39148800 & -0.00037700 \\
\hline $\mathrm{H}$ & -3.47705700 & -2.59135500 & 1.61151700 \\
\hline $\mathrm{H}$ & -2.46286100 & -3.46975300 & -2.52852400 \\
\hline $\mathrm{H}$ & -3.70916300 & -1.09606300 & -2.47098500 \\
\hline $\mathrm{H}$ & -6.27638800 & -0.20180400 & 0.34844700 \\
\hline $\mathrm{H}$ & -4.40403600 & 0.43579000 & 1.72101400 \\
\hline $\mathrm{H}$ & -4.57490500 & 1.22395600 & -0.57957100 \\
\hline $\mathrm{H}$ & 3.71046200 & 1.29841900 & -2.06410100 \\
\hline $\mathrm{H}$ & 4.48737000 & 0.71804700 & 0.16813300 \\
\hline $\mathrm{H}$ & 5.76611500 & 0.09107700 & -1.76783400 \\
\hline $\mathrm{H}$ & 3.85777100 & -2.34384900 & 0.62365300 \\
\hline $\mathrm{H}$ & 2.39267300 & -4.38156900 & -0.31988000 \\
\hline $\mathrm{H}$ & 1.52728300 & -3.70792100 & -2.78410300 \\
\hline $\mathrm{H}$ & 2.49131500 & -1.26949800 & -3.37211600 \\
\hline $\mathrm{C}$ & 0.88544300 & 5.08930500 & -1.53232800 \\
\hline $\mathrm{C}$ & -0.33832100 & 5.64476800 & -1.14863200 \\
\hline $\mathrm{C}$ & -1.52162100 & 4.92550600 & -1.33633700 \\
\hline $\mathrm{C}$ & -1.48087000 & 3.65039300 & -1.90633600 \\
\hline $\mathrm{C}$ & -0.25680900 & 3.09370200 & -2.28736700 \\
\hline $\mathrm{C}$ & 0.92644900 & 3.81396400 & -2.10200700 \\
\hline $\mathrm{H}$ & 1.80525100 & 5.65070700 & -1.38968800 \\
\hline $\mathrm{H}$ & -0.36995000 & 6.63763700 & -0.70743100 \\
\hline $\mathrm{H}$ & -2.47381500 & 5.35920300 & -1.04164800 \\
\hline
\end{tabular}




$\begin{array}{cccc}\mathrm{H} & -2.40101200 & 3.08970800 & -2.05034300 \\ \mathrm{H} & -0.22215200 & 2.09575900 & -2.71587900 \\ \mathrm{H} & 1.87765200 & 3.37827900 & -2.39624900 \\ \mathrm{Ti} & -1.51047900 & -1.47247700 & -0.40917600 \\ \mathrm{H} & -0.15020900 & -0.26495200 & -0.72925300 \\ \mathrm{H} & -1.70848600 & 0.21119100 & 0.22051500\end{array}$

\section{TS1 $^{\mathrm{AF}}$}

Ti

Ti

$\mathrm{Si}$

$\mathrm{Si}$

$\mathrm{Si}$

C

C

C

C

C

C

C

C

C

C

C

C

C

C

C

$\mathrm{H}$

$\mathrm{H}$

$\mathrm{H}$

$\mathrm{H}$

$\mathrm{H}$

$\mathrm{H}$

$\mathrm{H}$

$\mathrm{H}$

$\mathrm{H}$

$\mathrm{H}$

$\mathrm{H}$

$\mathrm{H}$

$\mathrm{H}$

$\mathrm{H}$

$\mathrm{H}$

$\mathrm{H}$

$\mathrm{H}$

$\mathrm{H}$

$\begin{array}{ccc}0.28772700 & -1.72652400 & -0.62984200 \\ 0.72339900 & 0.46565500 & 0.94597400 \\ -5.06115800 & 1.12525200 & -0.98056500 \\ 3.47123000 & -1.47395400 & -2.61731300 \\ 3.06955800 & -1.32708100 & 3.27850700 \\ -4.38025900 & -0.18357900 & 0.17019600 \\ -4.03616400 & -0.01514000 & 1.54866800 \\ -3.59716800 & -1.25285000 & 2.07375100 \\ -3.65648900 & -2.21395800 & 1.03245500 \\ -4.13393300 & -1.56097100 & -0.13279500 \\ 2.06524200 & -2.54731200 & -2.01701600 \\ 2.03626700 & -3.33845000 & -0.82436600 \\ 0.80878000 & -4.04020400 & -0.76453500 \\ 0.05445000 & -3.68697600 & -1.91695200 \\ 0.82581900 & -2.77474800 & -2.68709800 \\ 2.45976700 & 0.29855100 & 2.58574600 \\ 1.40973100 & 1.10649600 & 3.11581000 \\ 1.27767000 & 2.27186400 & 2.31682700 \\ 2.25291400 & 2.20586600 & 1.28001800 \\ 2.97232000 & 0.99768900 & 1.44606000 \\ -1.56729300 & -1.40878500 & -1.17356000 \\ -1.00054400 & -2.00170000 & 0.67692200 \\ -1.08200200 & 0.36658100 & 1.61861000 \\ 0.98327200 & -1.33273800 & 1.04913400 \\ 1.37961500 & -0.17744500 & -0.68847800 \\ 0.54649200 & 3.05965600 & 2.45288500 \\ 0.78503800 & 0.85102900 & 3.96353100 \\ 2.39170000 & 2.93135400 & 0.48786300 \\ 3.75372300 & 0.63673100 & 0.78862100 \\ 3.68232500 & -2.15158200 & 2.20567100 \\ 4.11281900 & -1.08646100 & 4.31649200 \\ 1.95338500 & -2.06771700 & 3.92052300 \\ -3.22609900 & -1.42308500 & 3.07728700 \\ -4.06031300 & 0.92271600 & 2.09130600 \\ -3.36056700 & -3.25207100 & 1.10628300 \\ -4.27272200 & -2.02715700 & -1.10024300 \\ -6.55232800 & 1.07491900 & -0.99044800 \\ -4.65457600 & 2.47214000 & -0.50494800\end{array}$




$\begin{array}{lrrc}\mathrm{H} & -4.59022100 & 0.89614900 & -2.36927200 \\ \mathrm{H} & 2.95124500 & -0.34289000 & -3.42953200 \\ \mathrm{H} & 4.26241700 & -0.95344600 & -1.47183500 \\ \mathrm{H} & 4.39703800 & -2.26123100 & -3.48180000 \\ \mathrm{H} & 2.81271200 & -3.36558100 & -0.06932200 \\ \mathrm{H} & 0.48946700 & -4.69976300 & 0.03289000 \\ \mathrm{H} & -0.94395400 & -4.03251100 & -2.15539400 \\ \mathrm{H} & 0.51659000 & -2.31668300 & -3.61925600 \\ \mathrm{C} & 1.86676600 & 4.56623200 & -1.94451200 \\ \mathrm{C} & 1.19804400 & 5.33338600 & -0.98478200 \\ \mathrm{C} & -0.02074400 & 4.89264600 & -0.46324600 \\ \mathrm{C} & -0.57118600 & 3.68264400 & -0.89858600 \\ \mathrm{C} & 0.09800600 & 2.91469400 & -1.85791200 \\ \mathrm{C} & 1.31738300 & 3.35833300 & -2.38101200 \\ \mathrm{H} & 2.81212600 & 4.91313700 & -2.35394100 \\ \mathrm{H} & 1.62404400 & 6.27574000 & -0.65003000 \\ \mathrm{H} & -0.54562600 & 5.49499200 & 0.27420500 \\ \mathrm{H} & -1.52093700 & 3.33457300 & -0.50128400 \\ \mathrm{H} & -0.33435800 & 1.97642600 & -2.19305500 \\ \mathrm{H} & 1.83521500 & 2.76178700 & -3.12744300 \\ \mathrm{Ti} & -2.06224600 & -0.57936200 & 0.36706400 \\ \mathrm{H} & -0.50478600 & -0.05934800 & -0.36065400 \\ \mathrm{H} & -2.14636500 & 0.85303500 & -0.56112000\end{array}$

\section{$\mathrm{A2}^{\mathrm{AF}}$}

$\mathrm{Ti}$

Ti

$\mathrm{Si}$

$\mathrm{Si}$

$\mathrm{Si}$

C

C

C

C

C

C

C

C

C

C

C

C

C

C

C

$\begin{array}{ccc}1.65020000 & -1.15013100 & -0.48258700 \\ 0.11984900 & 0.88653300 & 0.60491400 \\ -4.67511100 & -1.17354800 & 0.14545800 \\ 4.79152800 & 0.83634900 & -0.24621200 \\ -0.33465400 & -0.00722100 & 4.14823600 \\ -3.29972600 & -2.37722600 & -0.25165000 \\ -2.48474600 & -3.07238000 & 0.69430100 \\ -1.58218100 & -3.90886400 & -0.00234700 \\ -1.82025600 & -3.74317100 & -1.39355400 \\ -2.87777800 & -2.81139000 & -1.54918400 \\ 3.99214200 & -0.82048600 & -0.56229000 \\ 3.75020800 & -1.85677900 & 0.39529500 \\ 3.19564100 & -2.97350400 & -0.27052600 \\ 3.09093300 & -2.65098900 & -1.65174500 \\ 3.57955800 & -1.33461000 & -1.83613000 \\ -0.07341400 & 1.34979900 & 2.89222700 \\ -1.07337900 & 2.12973300 & 2.22749000 \\ -0.43809600 & 3.07539900 & 1.38816900 \\ 0.96499100 & 2.90818800 & 1.51851400 \\ 1.19087900 & 1.85493600 & 2.43968800 \\ 0.33860300 & -1.95336500 & -1.67658200 \\ & \mathrm{~S} 112 & \end{array}$

S112 


\begin{tabular}{|c|c|c|c|}
\hline $\mathrm{H}$ & 0.38996600 & -2.26084200 & 0.27981100 \\
\hline $\mathrm{H}$ & -1.45759800 & -0.20509500 & 0.54169900 \\
\hline $\mathrm{H}$ & 1.13349500 & -0.50109300 & 1.17729500 \\
\hline $\mathrm{H}$ & 1.65784900 & 0.75923100 & -0.45875700 \\
\hline $\mathrm{H}$ & -0.93486600 & 3.76704000 & 0.71883200 \\
\hline $\mathrm{H}$ & -2.14425900 & 1.99152900 & 2.31866500 \\
\hline $\mathrm{H}$ & 1.72515600 & 3.46071500 & 0.98110100 \\
\hline $\mathrm{H}$ & 2.16008500 & 1.46823100 & 2.72720400 \\
\hline $\mathrm{H}$ & 0.89966800 & -0.82485500 & 4.26462300 \\
\hline $\mathrm{H}$ & -0.63677900 & 0.56892500 & 5.48989600 \\
\hline $\mathrm{H}$ & -1.48054900 & -0.87429100 & 3.76737900 \\
\hline $\mathrm{H}$ & -0.82273000 & -4.53698700 & 0.44357100 \\
\hline $\mathrm{H}$ & -2.51554100 & -2.93620100 & 1.76921900 \\
\hline $\mathrm{H}$ & -1.27396000 & -4.22377500 & -2.19472800 \\
\hline $\mathrm{H}$ & -3.29544200 & -2.48739300 & -2.49248400 \\
\hline $\mathrm{H}$ & -6.00225500 & -1.82748500 & -0.02795800 \\
\hline $\mathrm{H}$ & -4.55221800 & -0.72891900 & 1.55724200 \\
\hline $\mathrm{H}$ & -4.63237000 & 0.00562800 & -0.75916900 \\
\hline $\mathrm{H}$ & 4.39941900 & 1.80822300 & -1.29986000 \\
\hline $\mathrm{H}$ & 4.41357900 & 1.36346700 & 1.09198300 \\
\hline $\mathrm{H}$ & 6.27800100 & 0.71603000 & -0.27154500 \\
\hline $\mathrm{H}$ & 3.92932400 & -1.78557800 & 1.46161600 \\
\hline $\mathrm{H}$ & 2.87475800 & -3.89748800 & 0.19436400 \\
\hline $\mathrm{H}$ & 2.67539700 & -3.28850400 & -2.42218800 \\
\hline $\mathrm{H}$ & 3.62611100 & -0.80209700 & -2.77849800 \\
\hline $\mathrm{C}$ & 0.07642000 & 3.62398600 & -2.25937400 \\
\hline $\mathrm{C}$ & -0.60843800 & 4.80806700 & -1.98205900 \\
\hline $\mathrm{C}$ & -1.97581500 & 4.77229800 & -1.68370800 \\
\hline $\mathrm{C}$ & -2.65642600 & 3.55309700 & -1.65968100 \\
\hline $\mathrm{C}$ & -1.97266400 & 2.36476800 & -1.94070300 \\
\hline $\mathrm{C}$ & -0.60546500 & 2.39972600 & -2.24322800 \\
\hline $\mathrm{H}$ & 1.13648500 & 3.64808400 & -2.49812500 \\
\hline $\mathrm{H}$ & -0.08288800 & 5.75923400 & -2.00429100 \\
\hline $\mathrm{H}$ & -2.50903600 & 5.69649000 & -1.47589700 \\
\hline $\mathrm{H}$ & -3.71922800 & 3.52713800 & -1.43367300 \\
\hline $\mathrm{H}$ & -2.49604800 & 1.41313800 & -1.94350500 \\
\hline $\mathrm{H}$ & -0.08643500 & 1.48018000 & -2.49785000 \\
\hline $\mathrm{Ti}$ & -1.07782300 & -1.65010800 & -0.57457900 \\
\hline $\mathrm{H}$ & 0.05601300 & -0.26434900 & -0.86181800 \\
\hline $\mathrm{H}$ & -1.45967600 & -0.85512000 & -2.01978700 \\
\hline \multicolumn{4}{|c|}{$\mathrm{TS2}^{\mathrm{AF}}$} \\
\hline $\mathrm{Ti}$ & 1.73597700 & -0.96180600 & -0.52058000 \\
\hline $\mathrm{Ti}$ & -0.06710300 & 0.92771600 & 0.53361500 \\
\hline $\mathrm{Si}$ & -4.49630600 & -1.52391900 & 0.06236900 \\
\hline $\mathrm{Si}$ & 4.68918700 & 1.27020400 & -0.07584200 \\
\hline
\end{tabular}




\begin{tabular}{|c|c|c|c|}
\hline $\mathrm{Si}$ & -0.15201200 & -0.26653400 & 4.05449300 \\
\hline $\mathrm{C}$ & -3.01600600 & -2.61780800 & -0.26141600 \\
\hline $\mathrm{C}$ & -2.15115700 & -3.19156800 & 0.72364100 \\
\hline $\mathrm{C}$ & -1.17651900 & -3.98229500 & 0.07441400 \\
\hline $\mathrm{C}$ & -1.41282700 & -3.90393300 & -1.32532000 \\
\hline $\mathrm{C}$ & -2.54741000 & -3.07913900 & -1.53234600 \\
\hline $\mathrm{C}$ & 4.03789400 & -0.41595700 & -0.54097000 \\
\hline $\mathrm{C}$ & 3.88259800 & -1.54675600 & 0.32367300 \\
\hline $\mathrm{C}$ & 3.44278900 & -2.64920700 & -0.44468400 \\
\hline $\mathrm{C}$ & 3.32588900 & -2.22292900 & -1.79639600 \\
\hline $\mathrm{C}$ & 3.68995600 & -0.85729700 & -1.86112200 \\
\hline $\mathrm{C}$ & -0.23153500 & 1.18432300 & 2.88124400 \\
\hline $\mathrm{C}$ & -1.40005300 & 1.78543400 & 2.31882400 \\
\hline $\mathrm{C}$ & -1.01359200 & 2.88212000 & 1.51448600 \\
\hline $\mathrm{C}$ & 0.40359000 & 2.98953600 & 1.56849700 \\
\hline $\mathrm{C}$ & 0.88231500 & 1.95120300 & 2.40689000 \\
\hline $\mathrm{H}$ & 0.54051900 & -1.82210400 & -1.76843500 \\
\hline $\mathrm{H}$ & 0.68469900 & -2.32168500 & 0.10823700 \\
\hline $\mathrm{H}$ & -1.53981000 & -0.24480800 & 0.31649000 \\
\hline $\mathrm{H}$ & 0.79551400 & -0.56607300 & 1.07972100 \\
\hline $\mathrm{H}$ & 1.60964000 & 0.91312700 & -0.28484800 \\
\hline $\mathrm{H}$ & -1.67500500 & 3.50027200 & 0.92011200 \\
\hline $\mathrm{H}$ & -2.41526700 & 1.42951000 & 2.44762900 \\
\hline $\mathrm{H}$ & 1.00889100 & 3.71737300 & 1.04356500 \\
\hline $\mathrm{H}$ & 1.92312300 & 1.74636200 & 2.62367100 \\
\hline $\mathrm{H}$ & 1.22617100 & -0.81918800 & 4.07668200 \\
\hline $\mathrm{H}$ & -0.50201600 & 0.16035600 & 5.43963400 \\
\hline $\mathrm{H}$ & -1.11504800 & -1.32882900 & 3.66103400 \\
\hline $\mathrm{H}$ & -0.37341200 & -4.52458900 & 0.55487300 \\
\hline $\mathrm{H}$ & -2.20506700 & -3.00819600 & 1.79055100 \\
\hline $\mathrm{H}$ & -0.81727700 & -4.37104900 & -2.09907500 \\
\hline $\mathrm{H}$ & -2.98535200 & -2.84369500 & -2.49241700 \\
\hline $\mathrm{H}$ & -5.75812900 & -2.30924700 & -0.04862600 \\
\hline $\mathrm{H}$ & -4.41708800 & -0.96848800 & 1.43812800 \\
\hline $\mathrm{H}$ & -4.57073900 & -0.41486100 & -0.92479700 \\
\hline $\mathrm{H}$ & 4.19885200 & 2.29843500 & -1.03029100 \\
\hline $\mathrm{H}$ & 4.28120100 & 1.63316300 & 1.30784300 \\
\hline $\mathrm{H}$ & 6.17986600 & 1.28815600 & -0.11756300 \\
\hline $\mathrm{H}$ & 4.04710600 & -1.55080400 & 1.39496800 \\
\hline $\mathrm{H}$ & 3.20598200 & -3.63542700 & -0.06554700 \\
\hline $\mathrm{H}$ & 2.98020600 & -2.82875100 & -2.62455400 \\
\hline $\mathrm{H}$ & 3.69771900 & -0.24568700 & -2.75547500 \\
\hline $\mathrm{C}$ & -0.20565200 & 3.83056200 & -2.00579600 \\
\hline $\mathrm{C}$ & -1.15847100 & 4.81489000 & -1.74356900 \\
\hline $\mathrm{C}$ & -2.50849900 & 4.46530400 & -1.61183300 \\
\hline $\mathrm{C}$ & -2.90310100 & 3.13266300 & -1.73771400 \\
\hline
\end{tabular}




$\begin{array}{lccc}\mathrm{C} & -1.94987300 & 2.14158900 & -2.00376800 \\ \mathrm{C} & -0.59951100 & 2.49111300 & -2.14013500 \\ \mathrm{H} & 0.84134100 & 4.09941200 & -2.11992500 \\ \mathrm{H} & -0.85598200 & 5.85466200 & -1.65033700 \\ \mathrm{H} & -3.25019700 & 5.23577600 & -1.41712400 \\ \mathrm{H} & -3.95132000 & 2.86242600 & -1.64143900 \\ \mathrm{H} & -2.24968100 & 1.10542900 & -2.12808400 \\ \mathrm{H} & 0.13254000 & 1.72989900 & -2.39485700 \\ \mathrm{Ti} & -0.86665000 & -1.71271900 & -0.60297400 \\ \mathrm{H} & 0.08123800 & -0.16809200 & -0.97008200 \\ \mathrm{H} & -1.31927300 & -1.10057700 & -2.10488700\end{array}$

$\begin{array}{crcc}\mathbf{A 3}^{\mathbf{A F}} & & & \\ \mathrm{Ti} & -0.54318800 & -1.77824300 & -0.01288000 \\ \mathrm{Ti} & 1.01020600 & 0.55546400 & 0.66274100 \\ \mathrm{Si} & -3.95128400 & 2.97608800 & -1.40509500 \\ \mathrm{Si} & 0.32070200 & -3.23948300 & -3.31477300 \\ \mathrm{Si} & 2.74414300 & -1.71909800 & 3.02775900 \\ \mathrm{C} & -3.67850300 & 1.95550800 & 0.13504600 \\ \mathrm{C} & -2.94871700 & 2.33498500 & 1.30247500 \\ \mathrm{C} & -3.01649800 & 1.28497000 & 2.25585100 \\ \mathrm{C} & -3.79848300 & 0.24372800 & 1.69849400 \\ \mathrm{C} & -4.18692800 & 0.64186700 & 0.39452800 \\ \mathrm{C} & -0.25203200 & -3.53476900 & -1.56602400 \\ \mathrm{C} & 0.55903600 & -3.82049700 & -0.41809100 \\ \mathrm{C} & -0.27974700 & -4.04794600 & 0.70309700 \\ \mathrm{C} & -1.62257100 & -3.90544300 & 0.27209500 \\ \mathrm{C} & -1.60714700 & -3.59113800 & -1.11028100 \\ \mathrm{C} & 2.47004700 & 0.02765600 & 2.42908500 \\ \mathrm{C} & 1.52850400 & 0.97698400 & 2.93800200 \\ \mathrm{C} & 1.70247100 & 2.20823800 & 2.26004100 \\ \mathrm{C} & 2.74664400 & 2.04369000 & 1.31667500 \\ \mathrm{C} & 3.22076200 & 0.70995600 & 1.41703900 \\ \mathrm{H} & -1.96954200 & -1.23962400 & 0.97598200 \\ \mathrm{H} & -0.39174200 & -0.32943200 & 1.45881600 \\ \mathrm{H} & -0.24496400 & 0.01472500 & -0.61591200 \\ \mathrm{H} & -0.45112300 & 1.69325700 & 0.56148900 \\ \mathrm{H} & 1.24534500 & -1.21766900 & 0.13315500 \\ \mathrm{H} & 1.10893800 & 3.10236700 & 2.40254500 \\ \mathrm{H} & 0.78363900 & 0.77792000 & 3.69844700 \\ \mathrm{H} & 3.09145600 & 2.78859800 & 0.61086000 \\ \mathrm{H} & 4.01002600 & 0.27537300 & 0.81603500 \\ \mathrm{H} & 3.41505900 & -2.52021000 & 1.97100400 \\ \mathrm{H} & 3.61990800 & -1.72796500 & 4.23397900 \\ \mathrm{H} & 1.45610000 & -2.35947400 & 3.40254700 \\ & -2.53433900 & 1.27100000 & 3.22566100\end{array}$




$\begin{array}{lrrr}\mathrm{H} & -2.40685700 & 3.26369800 & 1.43015500 \\ \mathrm{H} & -4.02774400 & -0.70256000 & 2.16966400 \\ \mathrm{H} & -4.75934600 & 0.04127900 & -0.30194800 \\ \mathrm{H} & -5.15536900 & 3.84535000 & -1.24361500 \\ \mathrm{H} & -2.79112500 & 3.87257500 & -1.64807800 \\ \mathrm{H} & -4.18552900 & 2.09605100 & -2.57721300 \\ \mathrm{H} & -0.69412000 & -2.44629600 & -4.05560300 \\ \mathrm{H} & 1.61878900 & -2.50993100 & -3.31653100 \\ \mathrm{H} & 0.53195000 & -4.52074100 & -4.04713600 \\ \mathrm{H} & 1.64199600 & -3.84218900 & -0.40373500 \\ \mathrm{H} & 0.04793800 & -4.26155500 & 1.71332000 \\ \mathrm{H} & -2.50416000 & -3.98707600 & 0.89517700 \\ \mathrm{H} & -2.48166600 & -3.39306400 & -1.71753300 \\ \mathrm{C} & 3.53322700 & 1.13240900 & -2.25321900 \\ \mathrm{C} & 4.08418300 & 2.41206500 & -2.21644400 \\ \mathrm{C} & 3.24865500 & 3.53251400 & -2.10720200 \\ \mathrm{C} & 1.86641300 & 3.36949900 & -2.02978000 \\ \mathrm{C} & 1.30532600 & 2.08425500 & -2.07106800 \\ \mathrm{C} & 2.14137900 & 0.96439100 & -2.18529400 \\ \mathrm{H} & 4.17732100 & 0.26262900 & -2.35365500 \\ \mathrm{H} & 5.16106100 & 2.54332100 & -2.28280200 \\ \mathrm{H} & 3.68079800 & 4.52980000 & -2.08989500 \\ \mathrm{H} & 1.21686800 & 4.23721200 & -1.95201200 \\ \mathrm{H} & 0.22703600 & 1.95749200 & -2.04947600 \\ \mathrm{H} & 1.71148000 & -0.02911600 & -2.27412600 \\ \mathrm{Ti} & -1.83342700 & 0.48901600 & 0.35263700 \\ \mathrm{H} & -1.85023400 & 1.13232000 & -1.20551300 \\ \mathrm{H} & -1.92283700 & -0.85789700 & -0.91961700\end{array}$

$\begin{array}{lccc}\text { TS3 }^{\mathbf{A F}} & & & \\ \mathrm{Ti} & -1.26488900 & 1.47413600 & -0.09226500 \\ \mathrm{Ti} & -0.07986300 & -0.98259500 & 0.66505300 \\ \mathrm{Si} & 4.98854400 & 0.60073900 & -1.25940200 \\ \mathrm{Si} & -3.41981600 & 1.32233700 & -3.10904500 \\ \mathrm{Si} & -2.48013000 & -0.72164200 & 3.48692800 \\ \mathrm{C} & 3.88764500 & 1.58320500 & -0.12542200 \\ \mathrm{C} & 3.52352000 & 1.25570500 & 1.22144100 \\ \mathrm{C} & 2.71284700 & 2.29218700 & 1.74659000 \\ \mathrm{C} & 2.55851600 & 3.27745600 & 0.74159600 \\ \mathrm{C} & 3.26699400 & 2.84955400 & -0.40855000 \\ \mathrm{C} & -3.07583500 & 2.11447700 & -1.45315600 \\ \mathrm{C} & -3.60014300 & 1.72056200 & -0.17677400 \\ \mathrm{C} & -3.16160300 & 2.64084400 & 0.81200500 \\ \mathrm{C} & -2.35248300 & 3.60955100 & 0.17406100 \\ \mathrm{C} & -2.29489500 & 3.28706100 & -1.20721600 \\ \mathrm{C} & -1.04876900 & -1.62484500 & 2.70202100 \\ & & & \end{array}$




\begin{tabular}{|c|c|c|c|}
\hline $\mathrm{C}$ & 0.34566900 & -1.41538000 & 2.94753400 \\
\hline $\mathrm{C}$ & 1.09210800 & -2.37858900 & 2.22571000 \\
\hline $\mathrm{C}$ & 0.17757000 & -3.19417400 & 1.51534200 \\
\hline $\mathrm{C}$ & -1.13367800 & -2.73757900 & 1.80196100 \\
\hline $\mathrm{H}$ & 0.20105100 & 2.49047900 & -0.15699500 \\
\hline $\mathrm{H}$ & 0.03160600 & 0.82338800 & 1.22468600 \\
\hline $\mathrm{H}$ & 0.00771400 & 0.26890800 & -0.80762500 \\
\hline $\mathrm{H}$ & 1.63827600 & -0.57922000 & 0.29760800 \\
\hline $\mathrm{H}$ & -1.75714800 & -0.28288900 & 0.22395000 \\
\hline $\mathrm{H}$ & 2.17118300 & -2.44723000 & 2.18383200 \\
\hline $\mathrm{H}$ & 0.76508900 & -0.62789400 & 3.56154800 \\
\hline $\mathrm{H}$ & 0.43575400 & -3.98905500 & 0.82720700 \\
\hline $\mathrm{H}$ & -2.04810000 & -3.15160400 & 1.39500200 \\
\hline $\mathrm{H}$ & -3.69259200 & -0.85453700 & 2.63708700 \\
\hline $\mathrm{H}$ & -2.79418800 & -1.29025700 & 4.82777500 \\
\hline $\mathrm{H}$ & -2.15821100 & 0.71839000 & 3.67121000 \\
\hline $\mathrm{H}$ & 2.24949900 & 2.30573500 & 2.72597600 \\
\hline $\mathrm{H}$ & 3.80416600 & 0.35003300 & 1.74494800 \\
\hline $\mathrm{H}$ & 1.96336000 & 4.17805900 & 0.82187200 \\
\hline $\mathrm{H}$ & 3.33515000 & 3.38924400 & -1.34361700 \\
\hline $\mathrm{H}$ & 6.42809600 & 0.94793200 & -1.05145500 \\
\hline $\mathrm{H}$ & 4.85672100 & -0.85697400 & -0.99086900 \\
\hline $\mathrm{H}$ & 4.66932700 & 0.88762700 & -2.68195200 \\
\hline $\mathrm{H}$ & -2.28483100 & 1.57259500 & -4.03444200 \\
\hline $\mathrm{H}$ & -3.61384700 & -0.14316400 & -2.94023100 \\
\hline $\mathrm{H}$ & -4.65736300 & 1.87620800 & -3.72585900 \\
\hline $\mathrm{H}$ & -4.22725100 & 0.85659600 & 0.00590500 \\
\hline $\mathrm{H}$ & -3.37680800 & 2.58714400 & 1.87256900 \\
\hline $\mathrm{H}$ & -1.83118500 & 4.42633600 & 0.65715500 \\
\hline $\mathrm{H}$ & -1.71626600 & 3.82523900 & -1.94907000 \\
\hline $\mathrm{C}$ & -1.39552400 & -3.69918300 & -1.81925600 \\
\hline $\mathrm{C}$ & -0.67632800 & -4.89072600 & -1.74677000 \\
\hline $\mathrm{C}$ & 0.72410400 & -4.86961100 & -1.81254000 \\
\hline $\mathrm{C}$ & 1.40075000 & -3.65802200 & -1.94507300 \\
\hline $\mathrm{C}$ & 0.68217300 & -2.45612100 & -2.02598100 \\
\hline $\mathrm{C}$ & -0.71815700 & -2.47830700 & -1.96400100 \\
\hline $\mathrm{H}$ & -2.48179500 & -3.71310500 & -1.78278700 \\
\hline $\mathrm{H}$ & -1.20015700 & -5.83796100 & -1.64866700 \\
\hline $\mathrm{H}$ & 1.28156700 & -5.80160500 & -1.76543300 \\
\hline $\mathrm{H}$ & 2.48573300 & -3.63944300 & -1.99962900 \\
\hline $\mathrm{H}$ & 1.20837000 & -1.51607600 & -2.16237900 \\
\hline $\mathrm{H}$ & -1.28230200 & -1.55745000 & -2.07930000 \\
\hline $\mathrm{Ti}$ & 1.58496600 & 1.25220000 & -0.08125900 \\
\hline $\mathrm{H}$ & 2.12711100 & 0.57145400 & -1.52587400 \\
\hline $\mathrm{H}$ & 1.45028000 & 1.66234500 & -1.72565700 \\
\hline
\end{tabular}




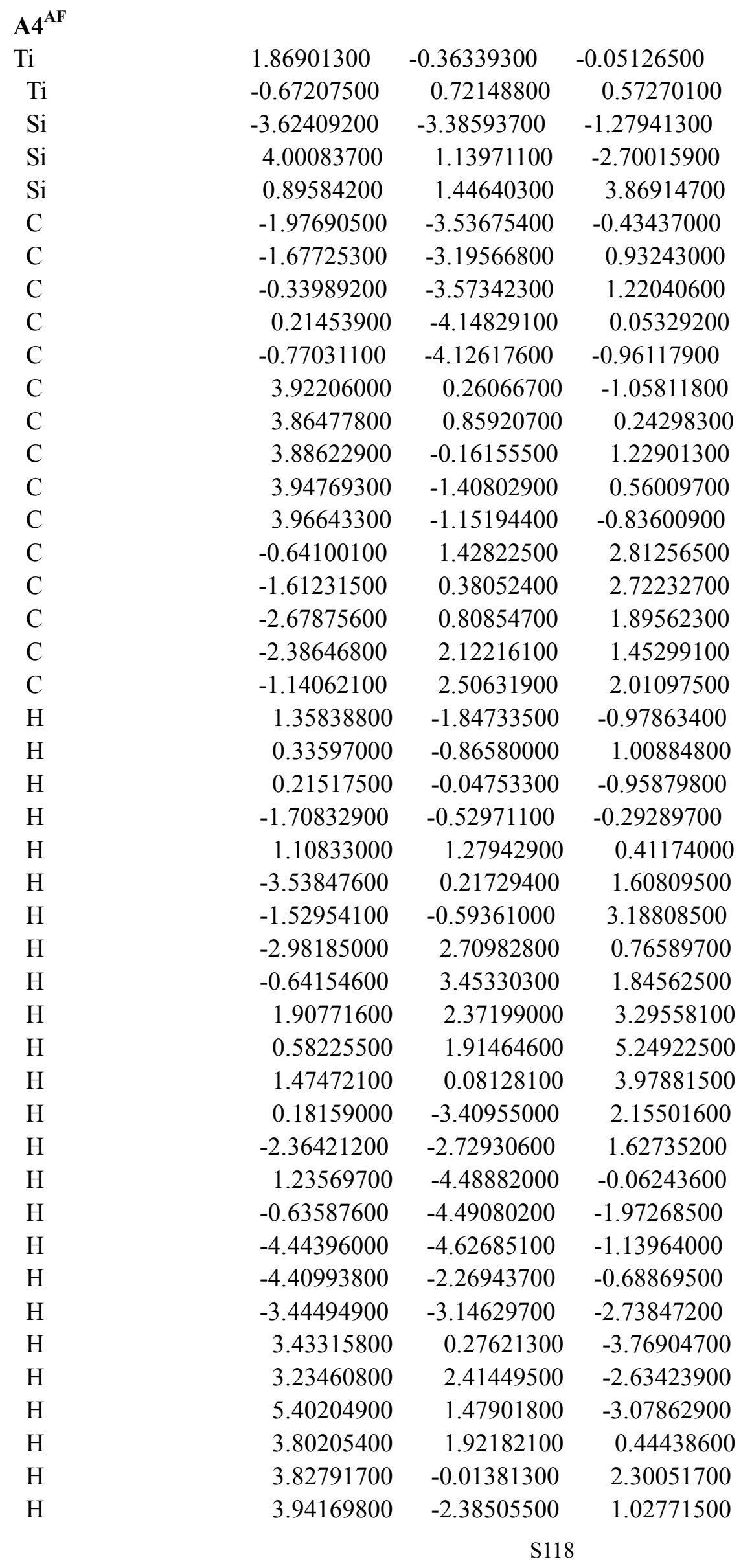




$\begin{array}{lccc}\mathrm{H} & 3.98653500 & -1.90895900 & -1.61030500 \\ \mathrm{C} & -0.95633100 & 4.19356600 & -1.35870700 \\ \mathrm{C} & -2.27194100 & 4.65480100 & -1.38538400 \\ \mathrm{C} & -3.30297800 & 3.81102500 & -1.81936200 \\ \mathrm{C} & -3.01677200 & 2.50724100 & -2.22263200 \\ \mathrm{C} & -1.69465300 & 2.04194300 & -2.20261900 \\ \mathrm{C} & -0.66258400 & 2.88571100 & -1.77137400 \\ \mathrm{H} & -0.15377700 & 4.85022300 & -1.03311400 \\ \mathrm{H} & -2.49799100 & 5.67212800 & -1.07690500 \\ \mathrm{H} & -4.32633400 & 4.17626900 & -1.84628000 \\ \mathrm{H} & -3.81421000 & 1.85185400 & -2.56198700 \\ \mathrm{H} & -1.46728200 & 1.03850400 & -2.54750400 \\ \mathrm{H} & 0.36672000 & 2.53976000 & -1.79132000 \\ \mathrm{Ti} & -0.40987400 & -1.86713600 & -0.45351300 \\ \mathrm{H} & -1.17967600 & -1.63028900 & -2.18763000 \\ \mathrm{H} & -0.42599300 & -1.81705900 & -2.38370600\end{array}$

\section{TS4 $^{\text {AF }}$}

Ti

Ti

$\mathrm{Si}$

$\mathrm{Si}$

$\mathrm{Si}$

C

C

C

C

C

C

C

C

C

C

C

C

C

C

C

$\mathrm{H}$

$\mathrm{H}$

$\mathrm{H}$

$\mathrm{H}$

$\mathrm{H}$

$\mathrm{H}$

$\mathrm{H}$

$\mathrm{H}$

$\begin{array}{ccc}-1.60505800 & -0.20312100 & -0.59439700 \\ 0.51477100 & 1.34745600 & 0.49519200 \\ 4.38737400 & -2.33625700 & -0.25788300 \\ -3.96105600 & -2.85133900 & 0.53797600 \\ -1.01434800 & 4.06167600 & -1.54659400 \\ 3.09219400 & -1.87667400 & -1.51780100 \\ 2.96981200 & -0.61221000 & -2.18798900 \\ 1.90755900 & -0.68497100 & -3.11937900 \\ 1.34977200 & -1.98634100 & -3.05086900 \\ 2.07012200 & -2.71720600 & -2.07479300 \\ -3.66427400 & -1.37003600 & -0.54370000 \\ -3.90049500 & 0.00295900 & -0.21072900 \\ -3.61130600 & 0.81097000 & -1.34582400 \\ -3.19246700 & -0.04717500 & -2.39612600 \\ -3.22063900 & -1.37366300 & -1.90748800 \\ 0.36216900 & 3.51118300 & -0.41188900 \\ 1.63876400 & 2.98679000 & -0.79393100 \\ 2.43558400 & 2.81946000 & 0.36437100 \\ 1.66922700 & 3.23098000 & 1.48089600 \\ 0.39730000 & 3.64972000 & 1.01326200 \\ -0.59730700 & -1.74328900 & -1.02096200 \\ 0.06795200 & 0.61821800 & -1.25737000 \\ -0.04430500 & -0.48561700 & 0.61003600 \\ 1.88568300 & 0.11168400 & 0.34316700 \\ -1.30905700 & 1.26130500 & 0.58818800 \\ 3.43674400 & 2.40918300 & 0.39588300 \\ 1.94013800 & 2.74118400 & -1.80467000 \\ 1.99226800 & 3.20748300 & 2.51486000\end{array}$

S119 


$\begin{array}{cccc}\mathrm{H} & -0.41644700 & 4.01552100 & 1.62816700 \\ \mathrm{H} & -2.30823600 & 4.06492500 & -0.81701300 \\ \mathrm{H} & -0.75890500 & 5.45210400 & -2.02242100 \\ \mathrm{H} & -1.10183400 & 3.18594200 & -2.74263900 \\ \mathrm{H} & 1.54768900 & 0.12588600 & -3.74035900 \\ \mathrm{H} & 3.57758200 & 0.26172000 & -1.99049500 \\ \mathrm{H} & 0.49442600 & -2.34288800 & -3.60953900 \\ \mathrm{H} & 1.86162800 & -3.73941300 & -1.77998500 \\ \mathrm{H} & 5.49143600 & -3.12568900 & -0.87346800 \\ \mathrm{H} & 4.97103900 & -1.10256800 & 0.33050000 \\ \mathrm{H} & 3.79876800 & -3.17050300 & 0.82594400 \\ \mathrm{H} & -3.02964000 & -3.95789400 & 0.18762000 \\ \mathrm{H} & -3.77284200 & -2.49121500 & 1.97157800 \\ \mathrm{H} & -5.34977400 & -3.38145900 & 0.40129600 \\ \mathrm{H} & -4.24135600 & 0.36934000 & 0.75066000 \\ \mathrm{H} & -3.68050700 & 1.89029500 & -1.39199500 \\ \mathrm{H} & -2.87645900 & 0.26104600 & -3.38541600 \\ \mathrm{H} & -2.91944200 & -2.25321600 & -2.46412000 \\ \mathrm{C} & -1.09507500 & 0.08027900 & 3.66650600 \\ \mathrm{C} & 0.04492400 & 0.88768300 & 3.54295000 \\ \mathrm{C} & -0.95822400 & -1.29428600 & 3.85126300 \\ \mathrm{H} & -2.08223100 & 0.53061600 & 3.62041000 \\ \mathrm{C} & 1.31959800 & 0.30999000 & 3.59418100 \\ \mathrm{H} & -0.06715300 & 1.96509500 & 3.45657200 \\ \mathrm{C} & 0.31693800 & -1.86878600 & 3.90950200 \\ \mathrm{H} & -1.84129900 & -1.91976000 & 3.94658500 \\ \mathrm{C} & 1.45320300 & -1.07167400 & 3.77676800 \\ \mathrm{H} & 2.20719900 & 0.93192300 & 3.51966900 \\ \mathrm{H} & 0.42058900 & -2.94038700 & 4.05756200 \\ \mathrm{H} & 2.44264300 & -1.51846900 & 3.82125200 \\ & 1.03884700 & -0.97639000 & -0.89419900 \\ \mathrm{H} & 0.33279600 & -3.60904900 & 0.71867600 \\ \mathrm{H} & 0.98420200 & -3.87593000 & 0.96262200 \\ & & & \\ \mathrm{H} & & & \\ \mathrm{H} & & \end{array}$

$\begin{array}{lrcc}\mathbf{A 5}^{\mathbf{A F}} & & & \\ \mathrm{Ti} & -1.40123700 & -0.30363700 & -0.68086800 \\ \mathrm{Ti} & 1.26904900 & -0.99692000 & -0.54746700 \\ \mathrm{Ti} & 0.42168900 & 1.28344000 & 0.78653600 \\ \mathrm{Si} & 4.43098200 & -2.19012700 & 0.81704900 \\ \mathrm{Si} & -4.40047400 & 1.93800200 & -0.56712500 \\ \mathrm{Si} & 2.82204500 & 3.74402100 & -0.61137300 \\ \mathrm{C} & 3.40575200 & -1.90069500 & -0.71759900 \\ \mathrm{C} & 3.39879000 & -0.71206500 & -1.52122100 \\ \mathrm{C} & 2.51316800 & -0.90206800 & -2.60854000 \\ \mathrm{C} & 1.95918500 & -2.20293700 & -2.50549800 \\ \mathrm{C} & 2.50288700 & -2.81990800 & -1.34997300\end{array}$




\begin{tabular}{|c|c|c|c|}
\hline $\mathrm{C}$ & -3.35857700 & 0.74719500 & -1.55915400 \\
\hline $\mathrm{C}$ & -2.23002800 & 1.08777100 & -2.37129300 \\
\hline $\mathrm{C}$ & -1.74537800 & -0.08538500 & -3.00321900 \\
\hline $\mathrm{C}$ & -2.56973400 & -1.17035700 & -2.59751200 \\
\hline $\mathrm{C}$ & -3.55343300 & -0.66009900 & -1.72123400 \\
\hline $\mathrm{C}$ & 1.65842400 & 3.29622100 & 0.76687800 \\
\hline $\mathrm{C}$ & 1.98423800 & 2.62229800 & 1.98949400 \\
\hline $\mathrm{C}$ & 0.82900500 & 2.54024400 & 2.80303400 \\
\hline $\mathrm{C}$ & -0.23922500 & 3.15177100 & 2.09555100 \\
\hline $\mathrm{C}$ & 0.26467000 & 3.61853800 & 0.85063400 \\
\hline $\mathrm{H}$ & -0.34678100 & -1.78953400 & -0.98063400 \\
\hline $\mathrm{H}$ & -0.12302200 & -0.64205300 & 0.71804600 \\
\hline $\mathrm{H}$ & 1.88710300 & 0.10397900 & 0.75162300 \\
\hline $\mathrm{H}$ & 0.29253200 & 0.57782000 & -1.05179200 \\
\hline $\mathrm{H}$ & -1.45152000 & 1.04333300 & 0.54490700 \\
\hline $\mathrm{H}$ & 0.76695000 & 2.07052600 & 3.77758800 \\
\hline $\mathrm{H}$ & 2.95555800 & 2.21076100 & 2.23745300 \\
\hline $\mathrm{H}$ & -1.26477500 & 3.23280700 & 2.43499100 \\
\hline $\mathrm{H}$ & -0.31269500 & 4.13053200 & 0.08965600 \\
\hline $\mathrm{H}$ & 2.09495400 & 3.80310600 & -1.90965000 \\
\hline $\mathrm{H}$ & 3.45431300 & 5.07961300 & -0.39996900 \\
\hline $\mathrm{H}$ & 3.92685400 & 2.75113300 & -0.71523900 \\
\hline $\mathrm{H}$ & 2.26693600 & -0.16242800 & -3.36079900 \\
\hline $\mathrm{H}$ & 3.94958200 & 0.19502400 & -1.30783800 \\
\hline $\mathrm{H}$ & 1.22159300 & -2.63656300 & -3.16847400 \\
\hline $\mathrm{H}$ & 2.26312000 & -3.81760400 & -0.99957600 \\
\hline $\mathrm{H}$ & 5.57190000 & -3.10487000 & 0.52990300 \\
\hline $\mathrm{H}$ & 4.97272200 & -0.89834600 & 1.30807800 \\
\hline $\mathrm{H}$ & 3.61057300 & -2.82802200 & 1.88368400 \\
\hline $\mathrm{H}$ & -4.80481900 & 1.33118100 & 0.73059800 \\
\hline $\mathrm{H}$ & -3.64372800 & 3.19136400 & -0.32322200 \\
\hline $\mathrm{H}$ & -5.65169400 & 2.28123500 & -1.30072400 \\
\hline $\mathrm{H}$ & -1.79702200 & 2.07605900 & -2.46035700 \\
\hline $\mathrm{H}$ & -0.88618500 & -0.14814600 & -3.65779500 \\
\hline $\mathrm{H}$ & -2.44530900 & -2.20705200 & -2.88381100 \\
\hline $\mathrm{H}$ & -4.32265800 & -1.24821300 & -1.23311300 \\
\hline $\mathrm{C}$ & -1.45207900 & -2.09782500 & 3.44146000 \\
\hline $\mathrm{C}$ & -1.15212400 & -3.31568600 & 2.81852900 \\
\hline $\mathrm{C}$ & -1.77471000 & -3.66211900 & 1.62058900 \\
\hline $\mathrm{C}$ & -2.70950200 & -2.79393800 & 1.04205200 \\
\hline $\mathrm{C}$ & -3.01239400 & -1.57683800 & 1.66453400 \\
\hline $\mathrm{C}$ & -2.37630400 & -1.22877600 & 2.86537900 \\
\hline $\mathrm{H}$ & -0.96415000 & -1.83176400 & 4.37525200 \\
\hline $\mathrm{H}$ & -0.43438400 & -3.99355600 & 3.27323400 \\
\hline $\mathrm{H}$ & -1.54464800 & -4.60835600 & 1.13877200 \\
\hline $\mathrm{H}$ & -3.21963800 & -3.08312100 & 0.12744100 \\
\hline
\end{tabular}




$\begin{array}{llll}\mathrm{H} & -3.77097100 & -0.91840100 & 1.25023800 \\ \mathrm{H} & -2.61123300 & -0.28248300 & 3.34402100\end{array}$

TS5 $^{\text {AF }}$

Ti

Ti

Ti

$\mathrm{Si}$

$\mathrm{Si}$

$\mathrm{Si}$

C

C

C

C

C

C

C

C

C

C

C

C

C

C

C

$\mathrm{H}$

$\mathrm{H}$

$\mathrm{H}$

$\mathrm{H}$

$\mathrm{H}$

$\mathrm{H}$

$\mathrm{H}$

$\mathrm{H}$

$\mathrm{H}$

$\mathrm{H}$

$\mathrm{H}$

$\mathrm{H}$

$\mathrm{H}$

$\mathrm{H}$

$\mathrm{H}$

$\mathrm{H}$

$\mathrm{H}$

$\mathrm{H}$

$\mathrm{H}$

$\mathrm{H}$

$\mathrm{H}$

\begin{tabular}{|c|c|c|}
\hline 1.37850700 & -0.80223200 & 0.45224000 \\
\hline-1.48104900 & -0.64274200 & 0.48277600 \\
\hline 0.01782100 & 1.24480000 & -0.95996400 \\
\hline-4.96687200 & -0.43075700 & -0.82883900 \\
\hline 4.91221000 & -0.36688300 & -0.70591400 \\
\hline 0.15402200 & 3.91310700 & 577600 \\
\hline-3.83989000 & -0.59146000 & 0.64749100 \\
\hline-3.29616900 & 0.48870000 & 1.42247700 \\
\hline-2.55838200 & -0.04679500 & 2.50528600 \\
\hline-2.63573400 & -1.463 & 585800 \\
\hline-3.41684100 & -1.79482700 & 1.29518300 \\
\hline 3.73908300 & 22000 & 131500 \\
\hline 3.107 & 8200 & 5700 \\
\hline 2.35 & 2800 & 2900 \\
\hline 2.50794800 & -1.76504700 & 4100 \\
\hline 3.35213500 & 1600 & 400100 \\
\hline 0.11 & 8100 & 900 \\
\hline-1.05281600 & 7300 & 3700 \\
\hline-0.652 & 5600 & 8900 \\
\hline 0.77 & 2900 & 800 \\
\hline 1.235 & 9100 & 1700 \\
\hline-0.09 & 900 & 800 \\
\hline-0.06 & -0.5 & -0.7 \\
\hline-1.690 & 1300 & 5900 \\
\hline-0.03 & 800 & 3200 \\
\hline 1.66 & 6100 & 6800 \\
\hline-1.314 & 54800 & 7600 \\
\hline-2.07 & 7100 & 800 \\
\hline 1.39 & 3100 & 5600 \\
\hline 2.272 & 11100 & -0.68037000 \\
\hline 1.348 & 6400 & 1700 \\
\hline & 6100 & 7000 \\
\hline-1.080 & 5400 & 14900 \\
\hline-2.002 & 0.52043700 & 3.24111200 \\
\hline-3.41254700 & 1.54163100 & 1.19934800 \\
\hline-2.15000800 & 56400 & 9398100 \\
\hline-3.64550900 & -2.80147700 & 0.96281100 \\
\hline-6.39890100 & -0.53859200 & -0.42826100 \\
\hline-4.77127800 & 0.88982200 & -1.48036900 \\
\hline-4.70479800 & -1.51580500 & -1.81476500 \\
\hline 4.75043900 & -1.38667000 & -1.78002200 \\
\hline 4.67874600 & 0.98810000 & -1.27008600 \\
\hline
\end{tabular}




$\begin{array}{lrrr}\mathrm{H} & 6.33438200 & -0.43136300 & -0.25941400 \\ \mathrm{H} & 3.19200600 & 1.36719400 & 1.40980100 \\ \mathrm{H} & 1.74404500 & 0.11274200 & 3.29448200 \\ \mathrm{H} & 2.02824100 & -2.54178800 & 2.93088500 \\ \mathrm{H} & 3.63570500 & -2.92612400 & 0.82522600 \\ \mathrm{C} & -0.60903000 & -1.97794700 & -2.80642400 \\ \mathrm{C} & -1.35560300 & -2.53993100 & -1.72184300 \\ \mathrm{C} & -0.68866400 & -3.06104800 & -0.62121700 \\ \mathrm{C} & 0.74187600 & -2.98787400 & -0.52396200 \\ \mathrm{C} & 1.46908300 & -2.37864400 & -1.57795000 \\ \mathrm{C} & 0.76762200 & -1.90814200 & -2.72726000 \\ \mathrm{H} & -1.13296800 & -1.62102300 & -3.68826500 \\ \mathrm{H} & -2.43198800 & -2.65375700 & -1.81122200 \\ \mathrm{H} & -1.23580900 & -3.60831000 & 0.14166600 \\ \mathrm{H} & 1.23969900 & -3.60181500 & 0.21845800 \\ \mathrm{H} & 2.54871600 & -2.47081600 & -1.62540600 \\ \mathrm{H} & 1.34148700 & -1.50234500 & -3.55721200\end{array}$

\begin{tabular}{|c|c|c|c|}
\hline$A 6^{\mathrm{AF}}$ & & & \\
\hline $\mathrm{Ti}$ & 1.57473900 & -0.96200000 & 0.24337000 \\
\hline $\mathrm{Ti}$ & -1.38896000 & -1.02005700 & 0.31176300 \\
\hline $\mathrm{Ti}$ & -0.13095200 & 1.33105800 & -0.52396400 \\
\hline $\mathrm{Si}$ & -4.92591700 & -0.33724800 & -0.68899800 \\
\hline $\mathrm{Si}$ & 4.95934900 & 0.60002900 & -0.22982500 \\
\hline $\mathrm{Si}$ & -0.76266300 & 3.46029600 & 2.45738600 \\
\hline $\mathrm{C}$ & -3.75430400 & -1.22752500 & 0.45789400 \\
\hline $\mathrm{C}$ & -3.24570100 & -0.73123500 & 1.70356600 \\
\hline $\mathrm{C}$ & -2.43341400 & -1.72764900 & 2.29672000 \\
\hline $\mathrm{C}$ & -2.43067100 & -2.86054100 & 1.43365200 \\
\hline $\mathrm{C}$ & -3.23873300 & -2.55346900 & 0.31538900 \\
\hline $\mathrm{C}$ & 3.92969100 & -0.68908300 & 0.63941400 \\
\hline $\mathrm{C}$ & 3.20068000 & -0.50934300 & 1.86094200 \\
\hline $\mathrm{C}$ & 2.58424100 & -1.73595900 & 2.21266600 \\
\hline $\mathrm{C}$ & 2.92311700 & -2.69421700 & 1.21458500 \\
\hline $\mathrm{C}$ & 3.74550000 & -2.05219800 & 0.26222900 \\
\hline $\mathrm{C}$ & -0.46637000 & 3.38870800 & 0.61475600 \\
\hline $\mathrm{C}$ & -1.46096300 & 3.28437400 & -0.40760100 \\
\hline $\mathrm{C}$ & -0.82346400 & 3.28580700 & -1.67336100 \\
\hline $\mathrm{C}$ & 0.57910000 & 3.39750900 & -1.45476500 \\
\hline $\mathrm{C}$ & 0.79392800 & 3.46331600 & -0.05555200 \\
\hline $\mathrm{H}$ & 0.12207900 & -1.82675200 & 0.97770800 \\
\hline $\mathrm{H}$ & 0.05521500 & -0.40291400 & -0.45244800 \\
\hline $\mathrm{H}$ & -1.79435700 & 0.57320600 & -0.48900200 \\
\hline $\mathrm{H}$ & -0.49469300 & 0.46768400 & 1.15481700 \\
\hline $\mathrm{H}$ & 1.55141200 & 0.87057000 & 0.05409700 \\
\hline $\mathrm{H}$ & -1.31747700 & 3.20553000 & -2.63409100 \\
\hline
\end{tabular}




$\begin{array}{lrrr}\mathrm{H} & -2.52614000 & 3.18367400 & -0.24116900 \\ \mathrm{H} & 1.34780600 & 3.42000800 & -2.21749100 \\ \mathrm{H} & 1.76116400 & 3.52408700 & 0.42733800 \\ \mathrm{H} & 0.37355500 & 2.84863700 & 3.19420600 \\ \mathrm{H} & -0.88833200 & 4.87446600 & 2.91437200 \\ \mathrm{H} & -2.02865400 & 2.76210500 & 2.80195700 \\ \mathrm{H} & -1.88214800 & -1.63273000 & 3.22364900 \\ \mathrm{H} & -3.42198500 & 0.25900300 & 2.10485500 \\ \mathrm{H} & -1.88095600 & -3.77980700 & 1.59217400 \\ \mathrm{H} & -3.41687600 & -3.20951700 & -0.52951700 \\ \mathrm{H} & -6.33307000 & -0.77077100 & -0.45730700 \\ \mathrm{H} & -4.85195200 & 1.12814900 & -0.45650800 \\ \mathrm{H} & -4.60126600 & -0.63475900 & -2.11115300 \\ \mathrm{H} & 4.83653900 & 0.46975200 & -1.70776100 \\ \mathrm{H} & 4.52975800 & 1.96008500 & 0.18749400 \\ \mathrm{H} & 6.40343700 & 0.45149200 & 0.10871600 \\ \mathrm{H} & 3.11684100 & 0.42051300 & 2.41011800 \\ \mathrm{H} & 1.95093400 & -1.90986800 & 3.07297700 \\ \mathrm{H} & 2.58236500 & -3.72151400 & 1.17555000 \\ \mathrm{H} & 4.13740300 & -2.51300500 & -0.63746300 \\ \mathrm{C} & -0.47642300 & -0.05092700 & -2.70396100 \\ \mathrm{C} & -1.12828500 & -1.29315400 & -2.26672600 \\ \mathrm{C} & -0.35431100 & -2.33310900 & -1.77712100 \\ \mathrm{C} & 1.07821700 & -2.20142900 & -1.59289300 \\ \mathrm{C} & 1.72168100 & -0.97732600 & -2.02535600 \\ \mathrm{C} & 0.90332500 & 0.06091800 & -2.62186000 \\ \mathrm{H} & -1.05738400 & 0.67399300 & -3.26509800 \\ \mathrm{H} & -2.17420600 & -1.44853600 & -2.51221500 \\ \mathrm{H} & -0.80811700 & -3.30538000 & -1.59848900 \\ \mathrm{H} & 1.65139100 & -3.12177600 & -1.53114100 \\ \mathrm{H} & 2.77491800 & -0.98077200 & -2.28848900 \\ & 1.40480300 & 0.90324700 & -3.09197600\end{array}$

\begin{tabular}{lccc} 
TS6 $^{\mathbf{A F}}$ & & & \\
$\mathrm{Ti}$ & 1.59680400 & -0.86815600 & 0.24178100 \\
$\mathrm{Ti}$ & -1.34967100 & -1.10462700 & 0.23010300 \\
$\mathrm{Ti}$ & -0.17093100 & 1.30153400 & -0.51353500 \\
$\mathrm{Si}$ & -4.91612000 & -0.24064500 & -0.53031100 \\
$\mathrm{Si}$ & 5.05577500 & 0.53813700 & -0.25355100 \\
$\mathrm{Si}$ & -0.87073700 & 3.25365000 & 2.55862600 \\
$\mathrm{C}$ & -3.72227800 & -1.31180800 & 0.42360200 \\
$\mathrm{C}$ & -3.16822500 & -1.03098900 & 1.71552400 \\
$\mathrm{C}$ & -2.34487600 & -2.11342400 & 2.10699900 \\
$\mathrm{C}$ & -2.37698300 & -3.08568800 & 1.06529800 \\
$\mathrm{C}$ & -3.22154500 & -2.59411900 & 0.04243700 \\
$\mathrm{C}$ & 3.94889500 & -0.66298500 & 0.64749800 \\
& & \multicolumn{2}{c}{$\mathrm{S} 124$} \\
\end{tabular}




\begin{tabular}{|c|c|c|c|}
\hline $\mathrm{C}$ & 3.23621500 & -0.40487100 & 1.86475200 \\
\hline $\mathrm{C}$ & 2.57032700 & -1.58542100 & 2.26855700 \\
\hline $\mathrm{C}$ & 2.86408200 & -2.59861100 & 1.31060600 \\
\hline $\mathrm{C}$ & 3.70698100 & -2.03496300 & 0.32749500 \\
\hline $\mathrm{C}$ & -0.58360200 & 3.28452300 & 0.71469700 \\
\hline $\mathrm{C}$ & -1.57894000 & 3.18481500 & -0.30912400 \\
\hline $\mathrm{C}$ & -0.95130900 & 3.29025300 & -1.57551300 \\
\hline $\mathrm{C}$ & 0.44386400 & 3.45744200 & -1.35549000 \\
\hline $\mathrm{C}$ & 0.66584200 & 3.45851700 & 0.04318000 \\
\hline $\mathrm{H}$ & 0.20181000 & -1.87456700 & 0.86889700 \\
\hline $\mathrm{H}$ & 0.08104200 & -0.39825500 & -0.45808400 \\
\hline $\mathrm{H}$ & -1.81599900 & 0.53027600 & -0.43967500 \\
\hline $\mathrm{H}$ & -0.57682600 & 0.42568700 & 1.12611600 \\
\hline $\mathrm{H}$ & 1.44775400 & 0.95621100 & 0.26362400 \\
\hline $\mathrm{H}$ & -1.44766700 & 3.24638400 & -2.53641100 \\
\hline $\mathrm{H}$ & -2.63743400 & 3.02951900 & -0.14296400 \\
\hline $\mathrm{H}$ & 1.20517400 & 3.55909300 & -2.11916600 \\
\hline $\mathrm{H}$ & 1.63173400 & 3.54654900 & 0.52441800 \\
\hline $\mathrm{H}$ & 0.28707200 & 2.64317800 & 3.26243000 \\
\hline $\mathrm{H}$ & -1.03598800 & 4.63897900 & 3.08743100 \\
\hline $\mathrm{H}$ & -2.11341100 & 2.50252400 & 2.87495400 \\
\hline $\mathrm{H}$ & -1.76650000 & -2.17732400 & 3.01984400 \\
\hline $\mathrm{H}$ & -3.32204300 & -0.11977800 & 2.28037700 \\
\hline $\mathrm{H}$ & -1.83068400 & -4.02058600 & 1.04957800 \\
\hline $\mathrm{H}$ & -3.43283100 & -3.09694700 & -0.89480700 \\
\hline $\mathrm{H}$ & -6.32212300 & -0.68485300 & -0.31085600 \\
\hline $\mathrm{H}$ & -4.80831500 & 1.17229400 & -0.08440600 \\
\hline $\mathrm{H}$ & -4.64699000 & -0.32769100 & -1.99160600 \\
\hline $\mathrm{H}$ & 4.94850800 & 0.35106500 & -1.72715000 \\
\hline $\mathrm{H}$ & 4.68112800 & 1.93301500 & 0.09603700 \\
\hline $\mathrm{H}$ & 6.48452800 & 0.33483900 & 0.11642600 \\
\hline $\mathrm{H}$ & 3.19084600 & 0.54986600 & 2.37392000 \\
\hline $\mathrm{H}$ & 1.92810000 & -1.69362600 & 3.13299000 \\
\hline $\mathrm{H}$ & 2.47885200 & -3.61058400 & 1.31753500 \\
\hline $\mathrm{H}$ & 4.08357100 & -2.55335200 & -0.54710300 \\
\hline $\mathrm{C}$ & -0.38023100 & 0.27416500 & -2.58140500 \\
\hline $\mathrm{C}$ & -1.05861200 & -1.00807800 & -2.38791900 \\
\hline $\mathrm{C}$ & -0.37889400 & -2.12540800 & -1.93213200 \\
\hline $\mathrm{C}$ & 1.07850900 & -2.07778700 & -1.73617800 \\
\hline $\mathrm{C}$ & 1.76818900 & -0.90543600 & -2.11904900 \\
\hline $\mathrm{C}$ & 1.05293800 & 0.28706700 & -2.45056700 \\
\hline $\mathrm{H}$ & -0.85584300 & 0.99581300 & -3.23764000 \\
\hline $\mathrm{H}$ & -2.10261400 & -1.08734800 & -2.67901400 \\
\hline $\mathrm{H}$ & -0.87670600 & -3.09029500 & -1.89452900 \\
\hline $\mathrm{H}$ & 1.61336500 & -3.01983800 & -1.66575700 \\
\hline $\mathrm{H}$ & 2.84407600 & -0.92207000 & -2.26470300 \\
\hline
\end{tabular}


$\mathrm{A} 7^{\mathrm{AF}}$

$\mathrm{Ti}$

Ti

$\mathrm{Ti}$

$\mathrm{Si}$

$\mathrm{Si}$

$\mathrm{Si}$

C

C

C

C

C

C

C

C

C

C

C

C

C

C

C

$\mathrm{H}$

$\mathrm{H}$

$\mathrm{H}$

$\mathrm{H}$

$\mathrm{H}$

$\mathrm{H}$

$\mathrm{H}$

$\mathrm{H}$

$\mathrm{H}$

$\mathrm{H}$

$\mathrm{H}$

$\mathrm{H}$

$\mathrm{H}$

$\mathrm{H}$

$\mathrm{H}$

$\mathrm{H}$

$\mathrm{H}$

$\mathrm{H}$

$\mathrm{H}$

$\mathrm{H}$

$\mathrm{H}$

$\mathrm{H}$

$\begin{array}{ccc}-1.43151300 & -1.01054400 & -0.26488200 \\ 1.56582500 & -0.70356000 & -0.38611500 \\ -0.08057100 & 1.34010200 & 0.56287800 \\ 4.96939600 & -0.92579700 & 1.11144700 \\ -5.07508600 & -0.46550400 & 0.51327500 \\ 0.37900300 & 3.60964900 & -2.33507300 \\ 3.88639900 & -1.10779500 & -0.39230300 \\ 3.60350600 & -0.09157200 & -1.36430600 \\ 2.82646500 & -0.65270900 & -2.40803700 \\ 2.61758000 & -2.02256600 & -2.10507200 \\ 3.25927300 & -2.30388300 & -0.87600100 \\ -3.80041800 & -1.22937400 & -0.61186700 \\ -3.23312300 & -0.62584800 & -1.78183300 \\ -2.35292100 & -1.54207300 & -2.39644100 \\ -2.36468600 & -2.73967000 & -1.62275800 \\ -3.25425300 & -2.54958300 & -0.54075600 \\ -0.03705500 & 3.44685100 & -0.52254500 \\ 0.88075100 & 3.49984500 & 0.57589400 \\ 0.15682000 & 3.38509200 & 1.78722100 \\ -1.22012700 & 3.25461600 & 1.46061800 \\ -1.33786000 & 3.30008300 & 0.05064900 \\ 0.10525900 & -1.64491700 & -1.07246200 \\ 0.05624400 & -0.34407100 & 0.34505200 \\ 1.69704400 & 0.90711900 & 0.53977400 \\ 0.67088600 & 0.84001900 & -1.06661200 \\ -1.44627400 & 0.79892700 & -0.48577200 \\ 0.58196500 & 3.38595000 & 2.78256400 \\ 1.95620800 & 3.59267100 & 0.49205300 \\ -2.03482600 & 3.12421300 & 2.16277100 \\ -2.26308600 & 3.21082400 & -0.50412900 \\ -0.54888700 & 2.80004400 & -3.16620000 \\ 0.24894600 & 5.02982700 & -2.77462300 \\ 1.78388900 & 3.19235500 & -2.57961900 \\ 2.42783200 & -0.11995300 & -3.26200000 \\ 3.90786800 & 0.94560900 & -1.29620800 \\ 2.03025500 & -2.71957900 & -2.68915700 \\ 3.26187100 & -3.26563800 & -0.37600600 \\ 6.37122200 & -1.34934600 & 0.83584400 \\ 4.99579500 & 0.49438500 & 1.55150600 \\ -6.44662300 & -0.95396400 & 0.19842600\end{array}$

S126 


$\begin{array}{lrrc}\mathrm{H} & -3.42525600 & 0.38425000 & -2.12084900 \\ \mathrm{H} & -1.75007500 & -1.35720300 & -3.27594600 \\ \mathrm{H} & -1.77763900 & -3.62828500 & -1.81820400 \\ \mathrm{H} & -3.47106200 & -3.28226900 & 0.22798300 \\ \mathrm{C} & 0.06239400 & 0.33038200 & 2.57678300 \\ \mathrm{C} & 1.17863200 & -0.54449300 & 2.43061800 \\ \mathrm{C} & 1.07544200 & -1.81158200 & 1.83033400 \\ \mathrm{C} & -0.26563600 & -2.35508700 & 1.64540400 \\ \mathrm{C} & -1.39506400 & -1.58902700 & 1.92157100 \\ \mathrm{C} & -1.26234900 & -0.14185100 & 2.10752400 \\ \mathrm{H} & 0.11819000 & 1.09164600 & 3.34815500 \\ \mathrm{H} & 2.13656100 & -0.22512500 & 2.83319200 \\ \mathrm{H} & 1.91726500 & -2.49536300 & 1.87266400 \\ \mathrm{H} & -0.37011900 & -3.42266600 & 1.46890900 \\ \mathrm{H} & -2.35388800 & -2.06888700 & 2.08874600 \\ \mathrm{H} & -2.13489600 & 0.36189600 & 2.51518000\end{array}$

\section{TS7 $^{\text {AF }}$}

Ti

Ti

Ti

$\mathrm{Si}$

$\mathrm{Si}$

$\mathrm{Si}$

C

C

C

C

C

C

C

C

C

C

C

C

C

C

C

$\mathrm{H}$

$\mathrm{H}$

$\mathrm{H}$

$\mathrm{H}$

$\mathrm{H}$

$\mathrm{H}$

$\mathrm{H}$

$\begin{array}{ccc}-1.48651300 & -0.93565400 & -0.24098400 \\ 1.52338900 & -0.77413300 & -0.37183100 \\ -0.00961900 & 1.37201900 & 0.49995900 \\ 4.92931500 & -1.07616800 & 1.15784100 \\ -5.14812200 & -0.47002300 & 0.55060700 \\ 0.58127200 & 3.64246600 & -2.37419900 \\ 3.84252400 & -1.31094400 & -0.33568500 \\ 3.60314600 & -0.35596000 & -1.37729400 \\ 2.79863200 & -0.95234200 & -2.38284100 \\ 2.51761300 & -2.27902400 & -1.97739000 \\ 3.14791000 & -2.49926500 & -0.72639500 \\ -3.85735600 & -1.18209700 & -0.58760600 \\ -3.29369900 & -0.53632600 & -1.73755900 \\ -2.41112200 & -1.42787300 & -2.38759300 \\ -2.41710200 & -2.64884500 & -1.65402900 \\ -3.30357800 & -2.50099600 & -0.56378100 \\ 0.16264100 & 3.48435000 & -0.56173200 \\ 1.09066400 & 3.47360300 & 0.53111900 \\ 0.37141200 & 3.41161400 & 1.74758300 \\ -1.01127100 & 3.36385200 & 1.43185900 \\ -1.13787200 & 3.41850800 & 0.02150900 \\ 0.03404900 & -1.82437200 & -0.84936100 \\ -0.03517600 & -0.04254800 & -0.49897700 \\ 1.71352500 & 0.75992700 & 0.63888500 \\ 1.04101400 & 0.99276200 & -1.02129400 \\ -1.50227400 & 0.91359200 & -0.46276500 \\ 0.80441100 & 3.38507800 & 2.73911700 \\ 2.16944000 & 3.49510700 & 0.43782100 \\ & \mathrm{~S} 127 & \\ & & \end{array}$




\begin{tabular}{lrrc}
$\mathrm{H}$ & -1.82752500 & 3.28244800 & 2.13961400 \\
$\mathrm{H}$ & -2.07243800 & 3.39395900 & -0.52426600 \\
$\mathrm{H}$ & -0.32823000 & 2.81592700 & -3.20933900 \\
$\mathrm{H}$ & 0.43601800 & 5.05851800 & -2.82165000 \\
$\mathrm{H}$ & 1.99251600 & 3.23921600 & -2.60362600 \\
$\mathrm{H}$ & 2.43544200 & -0.46603200 & -3.27941500 \\
$\mathrm{H}$ & 3.96274100 & 0.66602000 & -1.38693300 \\
$\mathrm{H}$ & 1.89801900 & -2.98969000 & -2.50944100 \\
$\mathrm{H}$ & 3.10198000 & -3.42092800 & -0.15755200 \\
$\mathrm{H}$ & 6.30488700 & -1.60155100 & 0.93059100 \\
$\mathrm{H}$ & 5.03766500 & 0.36996600 & 1.48747900 \\
$\mathrm{H}$ & 4.36812100 & -1.80324500 & 2.33132000 \\
$\mathrm{H}$ & -4.89629200 & -0.88325800 & 1.96026200 \\
$\mathrm{H}$ & -5.12574700 & 1.01514400 & 0.46955400 \\
$\mathrm{H}$ & -6.51863700 & -0.93164300 & 0.19308800 \\
$\mathrm{H}$ & -3.49814200 & 0.48112700 & -2.04652200 \\
$\mathrm{H}$ & -1.81807000 & -1.21419100 & -3.26764200 \\
$\mathrm{H}$ & -1.82912600 & -3.52910500 & -1.88132200 \\
$\mathrm{H}$ & -3.51065100 & -3.25972200 & 0.18234600 \\
$\mathrm{C}$ & -0.01716700 & 0.45014300 & 2.53126400 \\
$\mathrm{C}$ & 1.08199100 & -0.43670500 & 2.39326600 \\
$\mathrm{C}$ & 0.95066000 & -1.71454100 & 1.80888300 \\
$\mathrm{C}$ & -0.39896800 & -2.25434400 & 1.68625800 \\
$\mathrm{C}$ & -1.50937200 & -1.46054100 & 1.93079700 \\
$\mathrm{C}$ & -1.32284300 & -0.00814100 & 2.00568900 \\
$\mathrm{H}$ & 0.03290300 & 1.20889600 & 3.30513400 \\
$\mathrm{H}$ & 2.04070900 & -0.14165700 & 2.81163800 \\
$\mathrm{H}$ & 1.77877400 & -2.41240900 & 1.88718300 \\
$\mathrm{H}$ & -0.51340800 & -3.32844900 & 1.56523900 \\
$\mathrm{H}$ & -2.47948600 & -1.90476400 & 2.12807700 \\
$\mathrm{H}$ & -2.19223600 & 0.55418400 & 2.33816400 \\
& & & \\
\hline & & & \\
& & &
\end{tabular}

\begin{tabular}{lccc} 
TS11 $^{\mathbf{A F}}$ & & & \\
$\mathrm{Ti}$ & -0.31540800 & 1.26815000 & -0.61397400 \\
$\mathrm{Ti}$ & 1.64685800 & -0.40796800 & 0.42211300 \\
$\mathrm{Ti}$ & -1.21343800 & -1.29037400 & 0.16236000 \\
$\mathrm{Si}$ & 0.07959900 & 3.52201300 & 2.35627400 \\
$\mathrm{Si}$ & 4.97640300 & -1.23541900 & -1.19666800 \\
$\mathrm{Si}$ & -4.89121200 & -0.64733200 & -0.13818200 \\
$\mathrm{C}$ & 1.73981600 & 0.58877200 & -1.35686100 \\
$\mathrm{C}$ & 0.98002300 & 0.80692800 & -2.53361500 \\
$\mathrm{C}$ & -0.35937500 & 0.46307800 & -2.76334500 \\
$\mathrm{C}$ & -1.24452100 & -0.42866600 & -2.02298000 \\
$\mathrm{C}$ & -1.05627500 & -1.89624000 & -1.81756300 \\
$\mathrm{C}$ & 0.32152400 & -2.48933800 & -2.05977900 \\
$\mathrm{C}$ & -0.56438600 & 3.29933300 & 0.61931000 \\
& & \multicolumn{2}{c}{$\mathrm{S} 128$}
\end{tabular}




\begin{tabular}{|c|c|c|c|}
\hline $\mathrm{C}$ & 0.12547800 & 3.60528500 & -0.60043900 \\
\hline $\mathrm{C}$ & -0.74887100 & 3.38059500 & -1.68847100 \\
\hline $\mathrm{C}$ & -1.98404200 & 2.90995300 & -1.16751900 \\
\hline $\mathrm{C}$ & -1.87388600 & 2.87505600 & 0.24383200 \\
\hline $\mathrm{C}$ & 4.01794300 & -0.93852300 & 0.37728700 \\
\hline $\mathrm{C}$ & 3.86172500 & 0.30445400 & 1.06720700 \\
\hline $\mathrm{C}$ & 3.12985300 & 0.08659900 & 2.26062100 \\
\hline $\mathrm{C}$ & 2.82533100 & -1.29731200 & 2.33324300 \\
\hline $\mathrm{C}$ & 3.35802100 & -1.92322900 & 1.17922200 \\
\hline $\mathrm{C}$ & -2.85884700 & -1.25375500 & 1.90639400 \\
\hline $\mathrm{C}$ & -1.95830300 & -2.28095000 & 2.25291900 \\
\hline $\mathrm{C}$ & -2.04325100 & -3.29336600 & 1.25823300 \\
\hline $\mathrm{C}$ & -3.00833900 & -2.89015900 & 0.30820900 \\
\hline $\mathrm{C}$ & -3.52389100 & -1.60705000 & 0.68480100 \\
\hline $\mathrm{H}$ & 0.41222800 & -1.67514800 & 0.89480100 \\
\hline $\mathrm{H}$ & 0.72142200 & 1.07635200 & 0.87021800 \\
\hline $\mathrm{H}$ & 1.10535400 & -2.05915300 & -1.41311400 \\
\hline $\mathrm{H}$ & -1.37202800 & 0.45177200 & 0.55229800 \\
\hline $\mathrm{H}$ & 2.64974200 & 1.19905900 & -1.36597400 \\
\hline $\mathrm{H}$ & 1.37666000 & 1.51691900 & -3.26349000 \\
\hline $\mathrm{H}$ & -0.83240700 & 1.01247600 & -3.57664400 \\
\hline $\mathrm{H}$ & -2.28469100 & -0.20398900 & -2.27021600 \\
\hline $\mathrm{H}$ & -1.85309700 & -2.49241600 & -2.26485600 \\
\hline $\mathrm{H}$ & 0.31888000 & -3.57043200 & -1.88749400 \\
\hline $\mathrm{H}$ & -6.23424500 & -1.19754800 & 0.20010900 \\
\hline $\mathrm{H}$ & -4.85027300 & 0.76967000 & 0.31329300 \\
\hline $\mathrm{H}$ & -4.75826100 & -0.68981000 & -1.62134700 \\
\hline $\mathrm{H}$ & -3.00793200 & -0.33435100 & 2.45884800 \\
\hline $\mathrm{H}$ & -1.28733700 & -2.27946700 & 3.10316500 \\
\hline $\mathrm{H}$ & -1.45658200 & -4.20404600 & 1.22537700 \\
\hline $\mathrm{H}$ & -3.30477400 & -3.45758800 & -0.56466700 \\
\hline $\mathrm{H}$ & -2.65260900 & 2.55661200 & 0.92448600 \\
\hline $\mathrm{H}$ & -2.85307200 & 2.61850400 & -1.74490000 \\
\hline $\mathrm{H}$ & -0.50926400 & 3.51465900 & -2.73511900 \\
\hline $\mathrm{H}$ & 1.15276700 & 3.94071000 & -0.67539600 \\
\hline $\mathrm{H}$ & -0.49393200 & 2.49842100 & 3.26837100 \\
\hline $\mathrm{H}$ & 1.56353700 & 3.44021300 & 2.37196400 \\
\hline $\mathrm{H}$ & -0.30199800 & 4.86237300 & 2.88802100 \\
\hline $\mathrm{H}$ & 6.37548800 & -1.65655500 & -0.90010800 \\
\hline $\mathrm{H}$ & 4.34272900 & -2.31666000 & -1.99750400 \\
\hline $\mathrm{H}$ & 5.04259000 & 0.01390400 & -1.99951500 \\
\hline $\mathrm{H}$ & 3.27546400 & -2.97827200 & 0.94255200 \\
\hline $\mathrm{H}$ & 2.25140900 & -1.78415700 & 3.11246800 \\
\hline $\mathrm{H}$ & 2.84757000 & 0.84469800 & 2.98068800 \\
\hline $\mathrm{H}$ & 4.22276900 & 1.26643700 & 0.72165600 \\
\hline $\mathrm{H}$ & 0.67619900 & -2.29740600 & -3.08311400 \\
\hline
\end{tabular}




\begin{tabular}{|c|c|c|c|}
\hline$B 4^{\mathrm{AF}}$ & & & \\
\hline $\mathrm{Ti}$ & -0.26976900 & 1.42602200 & -0.65357100 \\
\hline $\mathrm{Ti}$ & 1.57511300 & -0.76266100 & 0.24038800 \\
\hline $\mathrm{Ti}$ & -1.34909600 & -0.99275000 & 0.14648200 \\
\hline $\mathrm{Si}$ & -0.10009100 & 3.11184400 & 2.69158800 \\
\hline $\mathrm{Si}$ & 5.10094900 & -1.06411700 & -1.03630600 \\
\hline $\mathrm{Si}$ & -5.00670900 & -0.96916800 & -0.57282100 \\
\hline $\mathrm{C}$ & 1.75704700 & 0.50945200 & -1.34005600 \\
\hline $\mathrm{C}$ & 1.04184500 & 0.83765600 & -2.52469000 \\
\hline $\mathrm{C}$ & -0.31034800 & 0.57311400 & -2.80085800 \\
\hline $\mathrm{C}$ & -1.24261100 & -0.31302900 & -2.12644000 \\
\hline $\mathrm{C}$ & -1.15811000 & -1.75300000 & -1.82838800 \\
\hline $\mathrm{C}$ & 0.11255600 & -2.50852300 & -2.18658300 \\
\hline $\mathrm{C}$ & -0.29306100 & 3.28884400 & 0.84639000 \\
\hline $\mathrm{C}$ & 0.74667700 & 3.52416500 & -0.10457500 \\
\hline $\mathrm{C}$ & 0.16579500 & 3.67497400 & -1.38815900 \\
\hline $\mathrm{C}$ & -1.24323700 & 3.54079000 & -1.25525200 \\
\hline $\mathrm{C}$ & -1.52240400 & 3.30957800 & 0.11193100 \\
\hline $\mathrm{C}$ & 3.93714800 & -1.13554300 & 0.42153600 \\
\hline $\mathrm{C}$ & 3.60195400 & -0.06339200 & 1.31044700 \\
\hline $\mathrm{C}$ & 2.76212600 & -0.55874300 & 2.33532200 \\
\hline $\mathrm{C}$ & 2.56442200 & -1.94518200 & 2.10321700 \\
\hline $\mathrm{C}$ & 3.27316800 & -2.29911100 & 0.92841200 \\
\hline $\mathrm{C}$ & -3.15131100 & -0.81833500 & 1.71224800 \\
\hline $\mathrm{C}$ & -2.15776100 & -1.59294000 & 2.34156300 \\
\hline $\mathrm{C}$ & -2.00893500 & -2.80120800 & 1.60098300 \\
\hline $\mathrm{C}$ & -2.91065700 & -2.76487000 & 0.51517700 \\
\hline $\mathrm{C}$ & -3.62229000 & -1.52084400 & 0.55442300 \\
\hline $\mathrm{H}$ & 0.15877500 & -1.92394200 & 0.56072400 \\
\hline $\mathrm{H}$ & 0.03076000 & 0.21264200 & 0.77269900 \\
\hline $\mathrm{H}$ & 1.03258500 & -2.02532400 & -1.82468800 \\
\hline $\mathrm{H}$ & -1.79313900 & 0.74349400 & 0.13940600 \\
\hline $\mathrm{H}$ & 2.67182500 & 1.10864100 & -1.26550500 \\
\hline $\mathrm{H}$ & 1.49356900 & 1.55410500 & -3.21458900 \\
\hline $\mathrm{H}$ & -0.74455000 & 1.17312000 & -3.60058900 \\
\hline $\mathrm{H}$ & -2.26917400 & -0.00446300 & -2.33961800 \\
\hline $\mathrm{H}$ & -2.05194000 & -2.29350000 & -2.14982900 \\
\hline $\mathrm{H}$ & 0.09592200 & -3.52682600 & -1.78560500 \\
\hline $\mathrm{H}$ & -6.28633000 & -1.63792900 & -0.20954900 \\
\hline $\mathrm{H}$ & -5.19181000 & 0.50036600 & -0.44933600 \\
\hline $\mathrm{H}$ & -4.70351400 & -1.31155400 & -1.98885100 \\
\hline $\mathrm{H}$ & -3.47792500 & 0.16462000 & 2.02645300 \\
\hline $\mathrm{H}$ & -1.58273300 & -1.30406200 & 3.21308100 \\
\hline $\mathrm{H}$ & -1.31428400 & -3.60057800 & 1.82428500 \\
\hline $\mathrm{H}$ & -3.03356200 & -3.54249700 & -0.22756400 \\
\hline
\end{tabular}




$\begin{array}{lrrr}\mathrm{H} & -2.50754900 & 3.15312800 & 0.53347500 \\ \mathrm{H} & -1.97054200 & 3.59813000 & -2.05606400 \\ \mathrm{H} & 0.70342900 & 3.84804800 & -2.31174400 \\ \mathrm{H} & 1.80640500 & 3.56130200 & 0.11577500 \\ \mathrm{H} & -1.15964800 & 2.22996700 & 3.25042700 \\ \mathrm{H} & 1.23655200 & 2.54915900 & 3.02280900 \\ \mathrm{H} & -0.21171800 & 4.43200900 & 3.37546400 \\ \mathrm{H} & 6.48750600 & -1.42853400 & -0.62589100 \\ \mathrm{H} & 4.67711000 & -2.02420600 & -2.08979900 \\ \mathrm{H} & 5.14598700 & 0.31304400 & -1.59424500 \\ \mathrm{H} & 3.31335100 & -3.29009800 & 0.49039900 \\ \mathrm{H} & 1.94778500 & -2.60864700 & 2.69731600 \\ \mathrm{H} & 2.32944100 & 0.02544500 & 3.13856500 \\ \mathrm{H} & 3.92130400 & 0.96683800 & 1.20408800 \\ \mathrm{H} & 0.23847500 & -2.56827900 & -3.27842100\end{array}$

\section{TS12 $^{\mathrm{AF}}$}

Ti

$\mathrm{Ti}$

$\mathrm{Ti}$

$\mathrm{Si}$

$\mathrm{Si}$

$\mathrm{Si}$

C

C

C

C

C

C

C

C

C

C

C

C

C

C

C

C

C

C

C

C

C

$\mathrm{H}$

$\mathrm{H}$

$\begin{array}{ccc}-0.32536600 & 1.33964800 & -0.72769000 \\ 1.53435600 & -0.68035200 & 0.33068800 \\ -1.20365400 & -1.03523000 & 0.27658500 \\ -0.99364700 & 3.44488200 & 2.24006400 \\ 5.11916900 & -0.80382300 & -0.87471300 \\ -4.80513700 & -1.05720500 & -0.89500800 \\ 1.53011700 & 0.32845800 & -1.53957400 \\ 0.48918300 & 0.25169600 & -2.47110400 \\ -0.77703900 & -0.45604100 & -2.17306200 \\ -0.95788300 & -1.84014500 & -1.79150300 \\ -0.02194300 & -2.64461300 & -1.11701800 \\ 1.46826100 & -2.75478200 & -1.33483100 \\ -0.66559800 & 3.36815700 & 0.40596200 \\ 0.60478200 & 3.46843200 & -0.25293100 \\ 0.40105000 & 3.46036500 & -1.65389300 \\ -0.99268800 & 3.35513900 & -1.89107000 \\ -1.64841100 & 3.29745800 & -0.63656500 \\ 3.92635000 & -0.60413900 & 0.54277900 \\ 3.41636900 & 0.63106300 & 1.05526100 \\ 2.63440900 & 0.36740400 & 2.20337500 \\ 2.65079700 & -1.03479200 & 2.43057700 \\ 3.43397600 & -1.63210200 & 1.41566900 \\ -3.20912500 & -0.72563000 & 1.55915800 \\ -2.30835300 & -1.44985000 & 2.36533500 \\ -2.09981000 & -2.71609100 & 1.74709200 \\ -2.88745500 & -2.76478700 & 0.57329900 \\ -3.57601500 & -1.52497800 & 0.42478700 \\ 0.22482100 & -1.64093700 & 1.21486800 \\ 0.01852900 & 0.33106900 & 0.80774200 \\ & & \end{array}$

S131 


$\begin{array}{lrrc}\mathrm{H} & 2.07333300 & -1.81916000 & -1.28042600 \\ \mathrm{H} & -1.81783400 & 0.66197100 & 0.00033000 \\ \mathrm{H} & 2.39293400 & 0.90633400 & -1.88683200 \\ \mathrm{H} & 0.54145600 & 0.76614700 & -3.43604300 \\ \mathrm{H} & -1.62355500 & -0.09359500 & -2.76583700 \\ \mathrm{H} & -1.92320000 & -2.27785000 & -2.03900600 \\ \mathrm{H} & -0.43003600 & -3.58946100 & -0.75799900 \\ \mathrm{H} & 1.90055000 & -3.51027800 & -0.67534300 \\ \mathrm{H} & -6.14659300 & -1.65009300 & -0.63247200 \\ \mathrm{H} & -4.95721900 & 0.42119300 & -0.94338600 \\ \mathrm{H} & -4.36164000 & -1.54749000 & -2.23059900 \\ \mathrm{H} & -3.55162900 & 0.28328700 & 1.75072400 \\ \mathrm{H} & -1.83553200 & -1.09856500 & 3.27369400 \\ \mathrm{H} & -1.44589800 & -3.49891700 & 2.11001700 \\ \mathrm{H} & -2.93502700 & -3.60065500 & -0.11538300 \\ \mathrm{H} & -2.71710800 & 3.20254100 & -0.48773200 \\ \mathrm{H} & -1.46973100 & 3.29249700 & -2.86274000 \\ \mathrm{H} & 1.17570500 & 3.48604400 & -2.41002100 \\ \mathrm{H} & 1.56736700 & 3.52920500 & 0.24087400 \\ \mathrm{H} & -2.24774100 & 2.72021200 & 2.57400900 \\ \mathrm{H} & 0.14277900 & 2.85295500 & 2.99524800 \\ \mathrm{H} & -1.14901900 & 4.85448400 & 2.70015300 \\ \mathrm{H} & 6.52622900 & -0.90790500 & -0.39460500 \\ \mathrm{H} & 4.82214500 & -2.04238100 & -1.64635300 \\ \mathrm{H} & 5.03309700 & 0.37195000 & -1.78138300 \\ \mathrm{H} & 3.63470800 & -2.69321400 & 1.31838400 \\ \mathrm{H} & 2.12520300 & -1.55630700 & 3.22051700 \\ \mathrm{H} & 2.09373100 & 1.10029200 & 2.78958900 \\ \mathrm{H} & 3.58599800 & 1.60717900 & 0.61713300 \\ & 1.68453900 & -3.04529000 & -2.37324200 \\ \mathrm{H} & & & \\ & & & \\ & & \end{array}$

$\begin{array}{cccc}\text { B5 }^{\mathbf{A F}} & & & \\ \mathrm{Ti} & -0.37815800 & 1.37442600 & -0.72552300 \\ \mathrm{Ti} & 1.52589400 & -0.84805600 & 0.24568900 \\ \mathrm{Ti} & -1.22120700 & -1.07258900 & 0.23929200 \\ \mathrm{Si} & -0.95367600 & 3.34555800 & 2.36652200 \\ \mathrm{Si} & 5.14543100 & -0.47773900 & -0.80744500 \\ \mathrm{Si} & -4.78118200 & -0.87130900 & -0.92401400 \\ \mathrm{C} & 1.45956700 & 0.36584700 & -1.56039500 \\ \mathrm{C} & 0.41805900 & 0.26556600 & -2.48088400 \\ \mathrm{C} & -0.82315200 & -0.45316500 & -2.13895700 \\ \mathrm{C} & -0.89526400 & -1.84407000 & -1.67919400 \\ \mathrm{C} & 0.25447400 & -2.40515300 & -0.97131100 \\ \mathrm{C} & 1.64403400 & -2.54973500 & -1.60632100 \\ \mathrm{C} & -0.63727200 & 3.35314200 & 0.52833100 \\ \mathrm{C} & 0.62810400 & 3.45322500 & -0.13668600 \\ & & & \end{array}$




\begin{tabular}{|c|c|c|c|}
\hline $\mathrm{C}$ & 0.41629900 & 3.51718900 & -1.53458400 \\
\hline $\mathrm{C}$ & -0.98176500 & 3.45675300 & -1.76542600 \\
\hline $\mathrm{C}$ & -1.62949400 & 3.35527800 & -0.50818200 \\
\hline $\mathrm{C}$ & 3.87720200 & -0.56209700 & 0.55653800 \\
\hline $\mathrm{C}$ & 3.24522300 & 0.55466200 & 1.18999700 \\
\hline $\mathrm{C}$ & 2.47046200 & 0.08902100 & 2.27539300 \\
\hline $\mathrm{C}$ & 2.62188700 & -1.32316500 & 2.34830700 \\
\hline $\mathrm{C}$ & 3.47655800 & -1.72713500 & 1.30138400 \\
\hline $\mathrm{C}$ & -3.22885000 & -0.85451500 & 1.58074100 \\
\hline $\mathrm{C}$ & -2.33643400 & -1.67157000 & 2.29642800 \\
\hline $\mathrm{C}$ & -2.13458900 & -2.86280100 & 1.53658400 \\
\hline $\mathrm{C}$ & -2.91701500 & -2.77175100 & 0.36321100 \\
\hline $\mathrm{C}$ & -3.58926100 & -1.51067700 & 0.35716300 \\
\hline $\mathrm{H}$ & 0.16963400 & -1.48512300 & 1.35476300 \\
\hline $\mathrm{H}$ & 0.06493100 & 0.24244400 & 0.66136700 \\
\hline $\mathrm{H}$ & 2.46676500 & -1.88317100 & -1.24038700 \\
\hline $\mathrm{H}$ & -1.81038700 & 0.66969600 & 0.09824300 \\
\hline $\mathrm{H}$ & 2.32600100 & 0.93435400 & -1.91260500 \\
\hline $\mathrm{H}$ & 0.44012900 & 0.79634400 & -3.43877700 \\
\hline $\mathrm{H}$ & -1.68278800 & -0.16931000 & -2.75414700 \\
\hline $\mathrm{H}$ & -1.64465800 & -2.48480200 & -2.14385000 \\
\hline $\mathrm{H}$ & 0.00139800 & -3.34876700 & -0.47960000 \\
\hline $\mathrm{H}$ & 2.02213700 & -3.56346600 & -1.44510400 \\
\hline $\mathrm{H}$ & -6.12654600 & -1.49745600 & -0.78375900 \\
\hline $\mathrm{H}$ & -4.94362300 & 0.59928100 & -0.77012800 \\
\hline $\mathrm{H}$ & -4.29787600 & -1.16912000 & -2.30071900 \\
\hline $\mathrm{H}$ & -3.55532600 & 0.13357700 & 1.87989000 \\
\hline $\mathrm{H}$ & -1.86153400 & -1.43005800 & 3.23880100 \\
\hline $\mathrm{H}$ & -1.48918600 & -3.68760300 & 1.81279700 \\
\hline $\mathrm{H}$ & -2.98230300 & -3.52833000 & -0.40868000 \\
\hline $\mathrm{H}$ & -2.69894600 & 3.27830000 & -0.35366500 \\
\hline $\mathrm{H}$ & -1.46746200 & 3.45547800 & -2.73482300 \\
\hline $\mathrm{H}$ & 1.18646700 & 3.56188100 & -2.29435700 \\
\hline $\mathrm{H}$ & 1.59645000 & 3.45916100 & 0.35004700 \\
\hline $\mathrm{H}$ & -2.25525400 & 2.69218800 & 2.66116900 \\
\hline $\mathrm{H}$ & 0.13852300 & 2.63249900 & 3.08126100 \\
\hline $\mathrm{H}$ & -1.00669600 & 4.73390800 & 2.90691000 \\
\hline $\mathrm{H}$ & 6.53019000 & -0.48607100 & -0.25789900 \\
\hline $\mathrm{H}$ & 5.02208600 & -1.64615200 & -1.72190300 \\
\hline $\mathrm{H}$ & 4.96973500 & 0.77623000 & -1.58750600 \\
\hline $\mathrm{H}$ & 3.78828300 & -2.74548900 & 1.09746800 \\
\hline $\mathrm{H}$ & 2.13444200 & -1.97577000 & 3.06145500 \\
\hline $\mathrm{H}$ & 1.84397400 & 0.69393200 & 2.91903800 \\
\hline $\mathrm{H}$ & 3.32723600 & 1.58582800 & 0.86921100 \\
\hline $\mathrm{H}$ & 1.62992000 & -2.32782300 & -2.67628100 \\
\hline
\end{tabular}




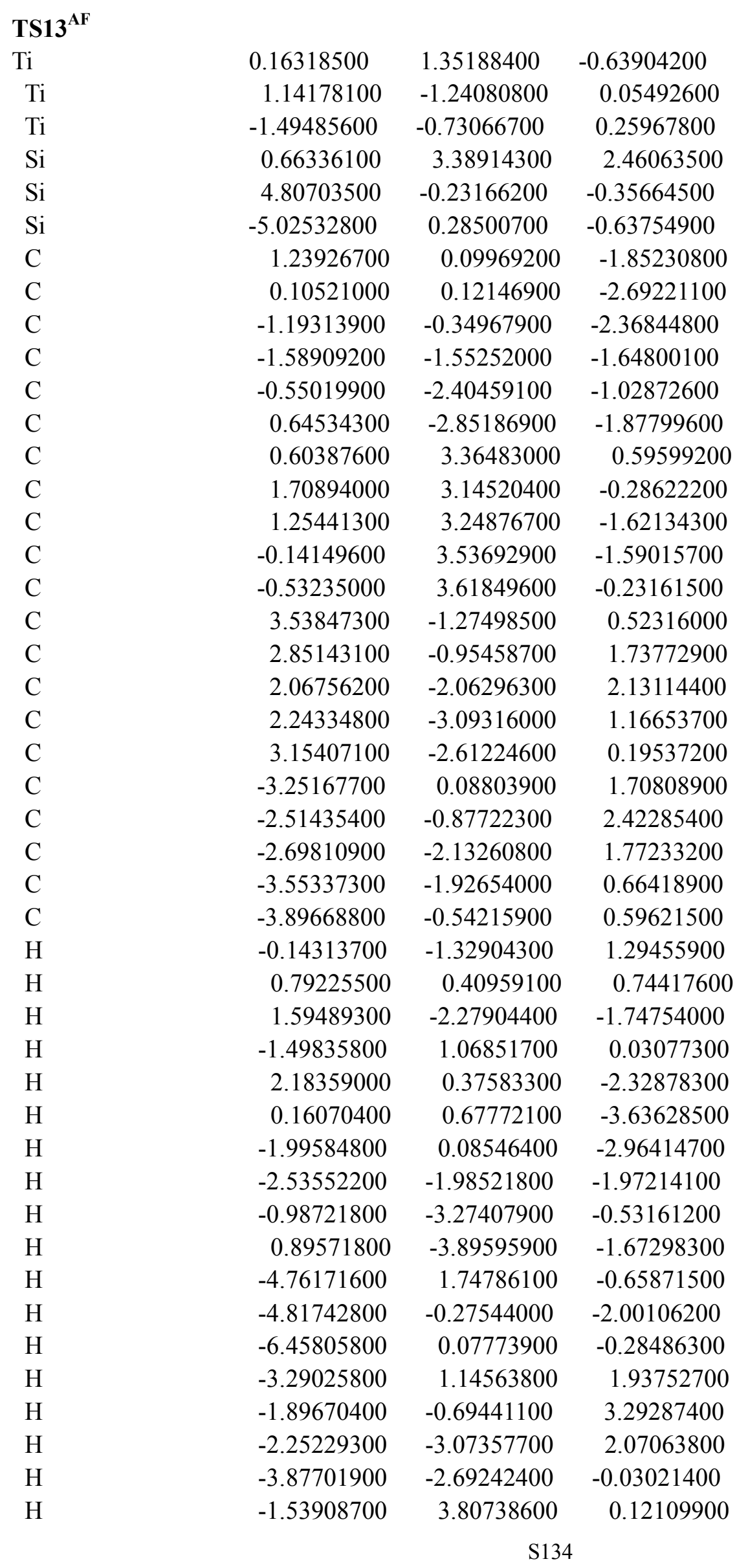




$\begin{array}{cccc}\mathrm{H} & -0.78766800 & 3.67440900 & -2.44892100 \\ \mathrm{H} & 1.85717900 & 3.11671800 & -2.51119700 \\ \mathrm{H} & 2.72093600 & 2.90758000 & 0.01872100 \\ \mathrm{H} & -0.59419900 & 2.84188000 & 3.03763100 \\ \mathrm{H} & 1.81890000 & 2.58801900 & 2.94491900 \\ \mathrm{H} & 0.82516300 & 4.77818400 & 2.97644600 \\ \mathrm{H} & 6.18662900 & -0.54316000 & 0.11331800 \\ \mathrm{H} & 4.76616000 & -0.48507000 & -1.82253000 \\ \mathrm{H} & 4.55804100 & 1.21135700 & -0.09976900 \\ \mathrm{H} & 3.50232500 & -3.16827900 & -0.66782300 \\ \mathrm{H} & 1.77626000 & -4.07053700 & 1.18173300 \\ \mathrm{H} & 1.43347300 & -2.11233500 & 3.00645300 \\ \mathrm{H} & 2.90887800 & -0.00927300 & 2.26279200 \\ \mathrm{H} & 0.45130300 & -2.71965000 & -2.94708700\end{array}$

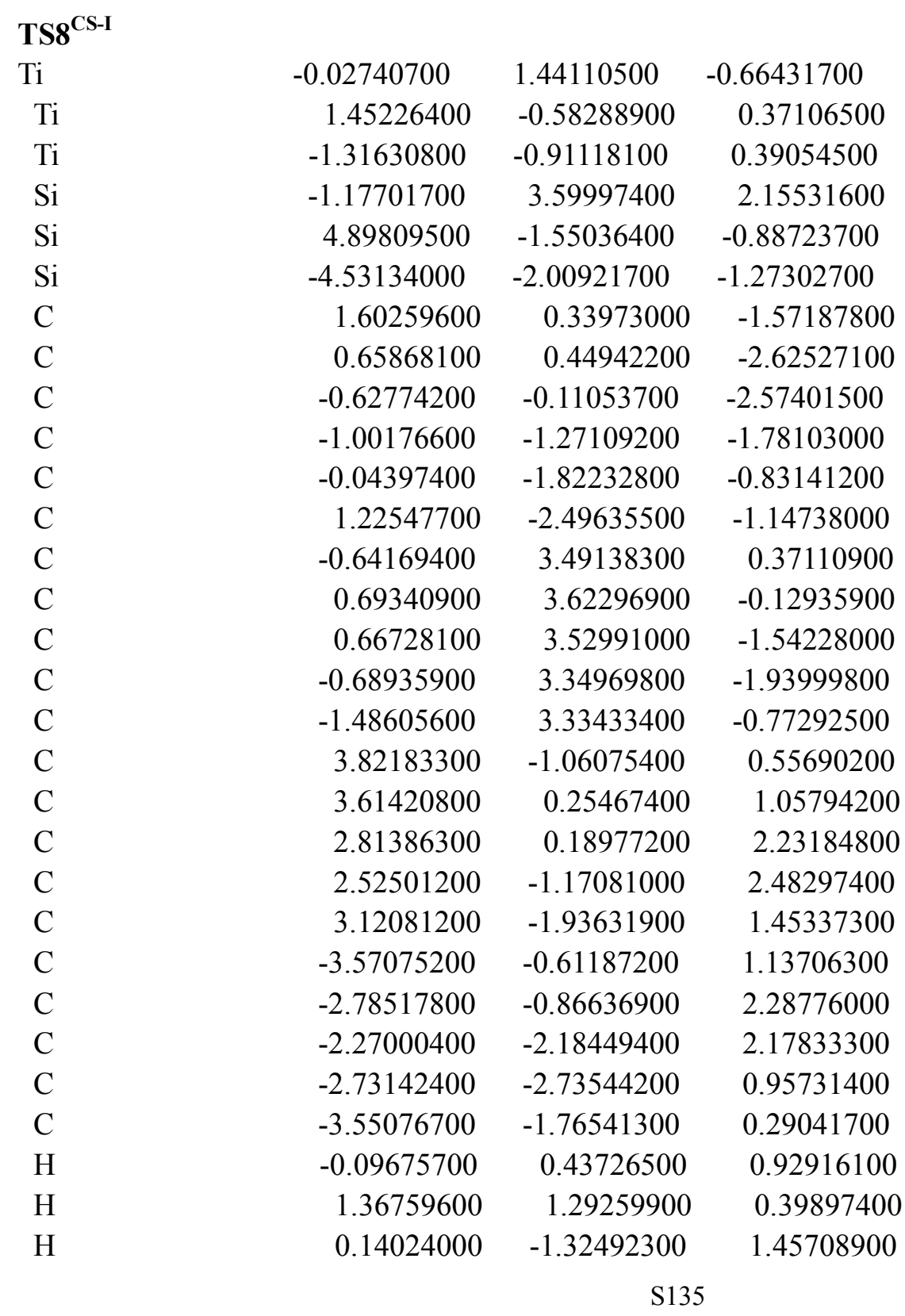




$\begin{array}{crrr}\mathrm{H} & -1.67895800 & 0.77832500 & -0.26659200 \\ \mathrm{H} & 2.56149400 & 0.82851300 & -1.76018100 \\ \mathrm{H} & 0.85227300 & 1.13613800 & -3.45325600 \\ \mathrm{H} & -1.35131300 & 0.23218500 & -3.31345700 \\ \mathrm{H} & -1.71622000 & -1.95334900 & -2.24841600 \\ \mathrm{H} & 0.10469000 & -3.10002100 & -0.90626200 \\ \mathrm{H} & 1.78015600 & -3.08083800 & -0.41645700 \\ \mathrm{H} & -5.91431100 & -2.48038300 & -0.97290400 \\ \mathrm{H} & -4.64609500 & -0.73688200 & -2.03432300 \\ \mathrm{H} & -3.89080400 & -3.04398400 & -2.13000400 \\ \mathrm{H} & -4.08746500 & 0.31555600 & 0.92272600 \\ \mathrm{H} & -2.58625300 & -0.16801800 & 3.09168500 \\ \mathrm{H} & -1.61691000 & -2.67304300 & 2.89010300 \\ \mathrm{H} & -2.51086500 & -3.73179800 & 0.59309300 \\ \mathrm{H} & -2.55811600 & 3.18550100 & -0.74750800 \\ \mathrm{H} & -1.04658500 & 3.22697200 & -2.95454000 \\ \mathrm{H} & 1.52637800 & 3.57067000 & -2.19962600 \\ \mathrm{H} & 1.58019800 & 3.76472300 & 0.47512400 \\ \mathrm{H} & -2.41252600 & 2.80472400 & 2.37973300 \\ \mathrm{H} & -0.09514800 & 3.10674900 & 3.04893200 \\ \mathrm{H} & -1.46986200 & 5.01053700 & 2.53805200 \\ \mathrm{H} & 6.29817900 & -1.80153700 & -0.44136900 \\ \mathrm{H} & 4.39296300 & -2.80851200 & -1.50017000 \\ \mathrm{H} & 4.93036700 & -0.46792400 & -1.90396000 \\ \mathrm{H} & 3.08667800 & -3.01762500 & 1.38198900 \\ \mathrm{H} & 1.92068400 & -1.55505000 & 3.29408700 \\ \mathrm{H} & 2.46981900 & 1.03496400 & 2.81444000 \\ & 3.97719700 & 1.16697200 & 0.60007200 \\ & 1.57813600 & -2.52941000 & -2.17434100\end{array}$

$\begin{array}{cccc}\text { TS1 }^{\text {CS-CH }} & & & \\ \mathrm{Ti} & -0.23652700 & 1.32521600 & 0.47855800 \\ \mathrm{Ti} & -1.10477300 & -0.95380400 & -0.57916200 \\ \mathrm{Ti} & 1.49050400 & -0.35650500 & -0.62018400 \\ \mathrm{Si} & -2.40829900 & 3.79013900 & -1.30396800 \\ \mathrm{Si} & -4.62920300 & -0.96415100 & 0.63792000 \\ \mathrm{Si} & 4.77634500 & 1.30663400 & 0.13177200 \\ \mathrm{C} & -0.03267800 & -1.38449000 & 3.05680500 \\ \mathrm{C} & 1.23371800 & -0.90697600 & 2.84874000 \\ \mathrm{C} & 2.13898200 & -1.60734900 & 1.99145800 \\ \mathrm{C} & 1.82851500 & -2.95537500 & 1.66464800 \\ \mathrm{C} & 0.56036600 & -3.44553500 & 1.86986900 \\ \mathrm{C} & -0.48045000 & -2.51049800 & 2.24937700 \\ \mathrm{C} & -1.33328700 & 3.41972600 & 0.18154400 \\ \mathrm{C} & -1.76220600 & 2.89510300 & 1.44300700 \\ \mathrm{C} & -0.64708000 & 2.82396300 & 2.31157100 \\ & & & \end{array}$




\begin{tabular}{|c|c|c|c|}
\hline $\mathrm{C}$ & 0.49091900 & 3.30441200 & 1.60961800 \\
\hline $\mathrm{C}$ & 0.07005500 & 3.67060100 & 0.30582600 \\
\hline $\mathrm{C}$ & -3.40075500 & -1.57404000 & -0.62879600 \\
\hline $\mathrm{C}$ & -3.08788500 & -0.96899800 & -1.88928800 \\
\hline $\mathrm{C}$ & -2.14698900 & -1.77722800 & -2.56730400 \\
\hline $\mathrm{C}$ & -1.86269100 & -2.90222800 & -1.74378400 \\
\hline $\mathrm{C}$ & -2.63534600 & -2.78254900 & -0.56281200 \\
\hline $\mathrm{C}$ & 3.06745100 & 0.53603500 & -2.14902700 \\
\hline $\mathrm{C}$ & 2.53973000 & -0.62848400 & -2.75262800 \\
\hline $\mathrm{C}$ & 2.93560500 & -1.74658500 & -1.96642500 \\
\hline $\mathrm{C}$ & 3.71569700 & -1.26237400 & -0.89135400 \\
\hline $\mathrm{C}$ & 3.80057600 & 0.16314500 & -0.97444200 \\
\hline $\mathrm{H}$ & -0.83103200 & -1.91813700 & 1.06983600 \\
\hline $\mathrm{H}$ & -1.34396800 & 0.86757700 & -0.85634500 \\
\hline $\mathrm{H}$ & 0.17766700 & -0.56660800 & -1.81978100 \\
\hline $\mathrm{H}$ & 0.85419400 & 1.32925900 & -0.95816600 \\
\hline $\mathrm{H}$ & -0.74374600 & -0.86016000 & 3.68890300 \\
\hline $\mathrm{H}$ & 1.57796800 & -0.00524300 & 3.35101400 \\
\hline $\mathrm{H}$ & 3.16394500 & -1.25710800 & 1.92256000 \\
\hline $\mathrm{H}$ & 2.60440100 & -3.59863600 & 1.25405200 \\
\hline $\mathrm{H}$ & 0.29860000 & -4.46254600 & 1.59140500 \\
\hline $\mathrm{H}$ & 6.20009200 & 1.38545500 & -0.30152500 \\
\hline $\mathrm{H}$ & 4.20547300 & 2.67811300 & 0.07648900 \\
\hline $\mathrm{H}$ & 4.76265300 & 0.81479900 & 1.53547600 \\
\hline $\mathrm{H}$ & 2.92626200 & 1.54815000 & -2.50682300 \\
\hline $\mathrm{H}$ & 1.92728500 & -0.66129300 & -3.64393900 \\
\hline $\mathrm{H}$ & 2.67152000 & -2.78042100 & -2.14955200 \\
\hline $\mathrm{H}$ & 4.16231200 & -1.87546900 & -0.11897800 \\
\hline $\mathrm{H}$ & 0.71215800 & 4.06301400 & -0.47314300 \\
\hline $\mathrm{H}$ & 1.50126000 & 3.36621800 & 1.99361500 \\
\hline $\mathrm{H}$ & -0.65496000 & 2.43853500 & 3.32500000 \\
\hline $\mathrm{H}$ & -2.76908800 & 2.57528000 & 1.68213100 \\
\hline $\mathrm{H}$ & -1.64814900 & 3.56838000 & -2.56114500 \\
\hline $\mathrm{H}$ & -3.62012200 & 2.93186900 & -1.28179500 \\
\hline $\mathrm{H}$ & -2.85116300 & 5.21328300 & -1.28467200 \\
\hline $\mathrm{H}$ & -5.99047500 & -1.51010300 & 0.37004600 \\
\hline $\mathrm{H}$ & -4.22641200 & -1.40866000 & 1.99866100 \\
\hline $\mathrm{H}$ & -4.72298500 & 0.51865900 & 0.60172100 \\
\hline $\mathrm{H}$ & -2.64332500 & -3.49023800 & 0.25658700 \\
\hline $\mathrm{H}$ & -1.16245800 & -3.69665600 & -1.96970100 \\
\hline $\mathrm{H}$ & -1.70197200 & -1.56389900 & -3.53089000 \\
\hline $\mathrm{H}$ & -3.48502100 & -0.02823800 & -2.25033200 \\
\hline $\mathrm{H}$ & -1.44470700 & -2.94087100 & 2.52193900 \\
\hline $\mathrm{H}$ & 0.47701200 & -1.82586800 & -0.40837400 \\
\hline $\mathrm{H}$ & -1.41191500 & 0.02538400 & 0.95704900 \\
\hline $\mathrm{H}$ & 1.44893300 & 0.77984800 & 0.81626000 \\
\hline
\end{tabular}




\begin{tabular}{|c|c|c|c|}
\hline \multicolumn{4}{|c|}{$\mathrm{A} 2^{\mathrm{CS}-\mathrm{CH}}$} \\
\hline $\mathrm{Ti}$ & -0.20620600 & 1.35860200 & -0.07116700 \\
\hline $\mathrm{Ti}$ & -1.02315500 & -1.16453300 & -0.74872800 \\
\hline $\mathrm{Ti}$ & 1.61742100 & -0.70015300 & -0.31740300 \\
\hline $\mathrm{Si}$ & -3.56741200 & 2.82675100 & -0.97020400 \\
\hline $\mathrm{Si}$ & -4.00576900 & -1.69674100 & 1.48637400 \\
\hline $\mathrm{Si}$ & 5.00754900 & 0.88612500 & 0.32415600 \\
\hline $\mathrm{C}$ & 0.10580300 & 1.27439700 & 2.16035500 \\
\hline $\mathrm{C}$ & 1.37600600 & 0.73474100 & 1.74802700 \\
\hline $\mathrm{C}$ & 1.74946400 & -0.64926900 & 1.93135500 \\
\hline $\mathrm{C}$ & 0.85957700 & -1.44583900 & 2.80369500 \\
\hline $\mathrm{C}$ & -0.25695200 & -0.95009700 & 3.35935100 \\
\hline $\mathrm{C}$ & -0.75438900 & 0.45739900 & 3.13527500 \\
\hline $\mathrm{C}$ & -1.73658900 & 3.09607000 & -0.72016700 \\
\hline $\mathrm{C}$ & -1.09295000 & 3.54588100 & 0.46807400 \\
\hline $\mathrm{C}$ & 0.28860900 & 3.71711700 & 0.19547900 \\
\hline $\mathrm{C}$ & 0.51186000 & 3.40941100 & -1.17151900 \\
\hline $\mathrm{C}$ & -0.71941900 & 3.01079900 & -1.73125100 \\
\hline $\mathrm{C}$ & -3.05509300 & -2.15786000 & -0.05732300 \\
\hline $\mathrm{C}$ & -3.26098800 & -1.64194000 & -1.37760700 \\
\hline $\mathrm{C}$ & -2.42868100 & -2.34699900 & -2.28105000 \\
\hline $\mathrm{C}$ & -1.69533000 & -3.31257000 & -1.54236200 \\
\hline $\mathrm{C}$ & -2.07468500 & -3.19561700 & -0.18129500 \\
\hline $\mathrm{C}$ & 3.45103300 & -0.36086900 & -1.83623800 \\
\hline $\mathrm{C}$ & 2.89236200 & -1.60602400 & -2.17407300 \\
\hline $\mathrm{C}$ & 3.08690500 & -2.48954900 & -1.07012500 \\
\hline $\mathrm{C}$ & 3.78437600 & -1.78825900 & -0.06823000 \\
\hline $\mathrm{C}$ & 3.99904600 & -0.44192100 & -0.51171800 \\
\hline $\mathrm{H}$ & -1.80249500 & 0.42753000 & 2.80700500 \\
\hline $\mathrm{H}$ & -1.57836500 & 0.50575900 & -1.09652700 \\
\hline $\mathrm{H}$ & 0.48913300 & -1.75572400 & -1.46785200 \\
\hline $\mathrm{H}$ & 0.24393100 & 0.06831900 & -1.29196300 \\
\hline $\mathrm{H}$ & 0.16627900 & 2.32155200 & 2.44761200 \\
\hline $\mathrm{H}$ & 2.18248600 & 1.44595800 & 1.58435900 \\
\hline $\mathrm{H}$ & 2.80794900 & -0.80039400 & 2.13425100 \\
\hline $\mathrm{H}$ & 1.15866900 & -2.47204800 & 3.01534900 \\
\hline $\mathrm{H}$ & -0.85438200 & -1.57708800 & 4.01951100 \\
\hline $\mathrm{H}$ & 6.46290300 & 0.69246500 & 0.07289400 \\
\hline $\mathrm{H}$ & 4.62182100 & 2.21948400 & -0.21066000 \\
\hline $\mathrm{H}$ & 4.79992700 & 0.86804400 & 1.79717500 \\
\hline $\mathrm{H}$ & 3.44866100 & 0.52333600 & -2.46147100 \\
\hline $\mathrm{H}$ & 2.37726600 & -1.84441000 & -3.09561500 \\
\hline $\mathrm{H}$ & 2.74298600 & -3.51500900 & -1.00957100 \\
\hline $\mathrm{H}$ & 4.08893200 & -2.19800900 & 0.88603100 \\
\hline $\mathrm{H}$ & -0.87287100 & 2.68060500 & -2.75138000 \\
\hline
\end{tabular}




$\begin{array}{crrr}\mathrm{H} & 1.46657800 & 3.43303400 & -1.68070200 \\ \mathrm{H} & 1.04708600 & 4.02181900 & 0.90678800 \\ \mathrm{H} & -1.57464500 & 3.71155600 & 1.42354800 \\ \mathrm{H} & -3.79825300 & 1.98670100 & -2.17515800 \\ \mathrm{H} & -4.18265300 & 2.17536900 & 0.21535600 \\ \mathrm{H} & -4.26244600 & 4.12780100 & -1.18405900 \\ \mathrm{H} & -5.26097400 & -2.49706000 & 1.56420900 \\ \mathrm{H} & -3.19503900 & -1.99740800 & 2.69189600 \\ \mathrm{H} & -4.38128100 & -0.26105900 & 1.45819200 \\ \mathrm{H} & -1.67497800 & -3.78385000 & 0.63539200 \\ \mathrm{H} & -0.95750700 & -3.99611500 & -1.94205000 \\ \mathrm{H} & -2.33835700 & -2.15578700 & -3.34476200 \\ \mathrm{H} & -3.93066700 & -0.83272900 & -1.64057600 \\ \mathrm{H} & -0.77925600 & 0.97945900 & 4.10815200 \\ \mathrm{H} & 0.20397100 & -1.71954300 & 0.38802200 \\ \mathrm{H} & -1.16748600 & -0.11456200 & 0.65254300 \\ \mathrm{H} & 1.50483000 & 1.08595200 & -0.67185100\end{array}$

$\begin{array}{cccc}\mathbf{A 1}^{\text {BS-CH }} & & & \\ \mathrm{Ti} & 0.14762300 & -0.31688100 & 1.01030600 \\ \mathrm{Ti} & 2.15110200 & 0.10743100 & -0.36919500 \\ \mathrm{Ti} & -0.15986900 & 1.50134500 & -1.12248400 \\ \mathrm{Si} & 0.31034900 & 0.48226800 & 4.62087400 \\ \mathrm{Si} & 3.65440800 & -3.08049200 & -1.49997200 \\ \mathrm{Si} & -2.58011000 & 3.44662200 & 1.00698000 \\ \mathrm{C} & -4.79337200 & -3.29298700 & -0.77742200 \\ \mathrm{C} & -5.03206100 & -1.93834600 & -1.02295000 \\ \mathrm{C} & -4.10249500 & -1.18640400 & -1.74645400 \\ \mathrm{C} & -2.93310000 & -1.78588500 & -2.22329900 \\ \mathrm{C} & -2.69647000 & -3.14151400 & -1.97864500 \\ \mathrm{C} & -3.62629900 & -3.89472000 & -1.25704300 \\ \mathrm{C} & -0.33081600 & -0.65921500 & 3.29161500 \\ \mathrm{C} & 0.31110600 & -1.84365100 & 2.80369600 \\ \mathrm{C} & -0.52766900 & -2.47009600 & 1.84524300 \\ \mathrm{C} & -1.69309200 & -1.68545300 & 1.70045400 \\ \mathrm{C} & -1.57022900 & -0.57149400 & 2.58092000 \\ \mathrm{C} & 3.92997900 & -1.30192300 & -1.00321700 \\ \mathrm{C} & 4.19529200 & -0.81041100 & 0.31910600 \\ \mathrm{C} & 4.42118500 & 0.59196500 & 0.26107400 \\ \mathrm{C} & 4.27895700 & 0.99602000 & -1.08369900 \\ \mathrm{C} & 3.96892600 & -0.15923200 & -1.85719800 \\ \mathrm{C} & -0.17241100 & 3.82221900 & -0.66697900 \\ \mathrm{C} & 0.23794900 & 3.64985700 & -2.01430800 \\ \mathrm{C} & -0.85376200 & 3.09735700 & -2.73921600 \\ \mathrm{C} & -1.92402800 & 2.92274100 & -1.82808500 \\ \mathrm{C} & -1.52027800 & 3.37547600 & -0.53254400\end{array}$




$\begin{array}{crrc}\mathrm{H} & 0.25526400 & -0.29722400 & -0.89555700 \\ \mathrm{H} & 1.86216300 & 0.09808100 & 1.44593600 \\ \mathrm{H} & 1.56610200 & 1.35887500 & -1.59999800 \\ \mathrm{H} & 0.96361800 & 1.42454300 & 0.37459600 \\ \mathrm{H} & -5.51704500 & -3.87972400 & -0.21761700 \\ \mathrm{H} & -5.94192300 & -1.47092800 & -0.65503800 \\ \mathrm{H} & -4.28967200 & -0.13276200 & -1.93884700 \\ \mathrm{H} & -2.20442900 & -1.19811300 & -2.77529700 \\ \mathrm{H} & -1.78805900 & -3.60803900 & -2.35090500 \\ \mathrm{H} & -3.24468400 & 4.77595500 & 1.11186700 \\ \mathrm{H} & -1.74705800 & 3.25156800 & 2.22048700 \\ \mathrm{H} & -3.63930700 & 2.40670500 & 0.94349700 \\ \mathrm{H} & 0.44886100 & 4.20132100 & 0.13511100 \\ \mathrm{H} & 1.21501600 & 3.88333000 & -2.41659800 \\ \mathrm{H} & -0.86187500 & 2.84781200 & -3.79091200 \\ \mathrm{H} & -2.88653600 & 2.48448400 & -2.06796600 \\ \mathrm{H} & -2.30264700 & 0.22013300 & 2.68778300 \\ \mathrm{H} & -2.51138900 & -1.87496400 & 1.01715000 \\ \mathrm{H} & -0.28938400 & -3.36449400 & 1.28347300 \\ \mathrm{H} & 1.29250800 & -2.19307100 & 3.10024800 \\ \mathrm{H} & -0.04320600 & 1.89415900 & 4.31468300 \\ \mathrm{H} & 1.78630900 & 0.34601100 & 4.72722600 \\ \mathrm{H} & -0.27970800 & 0.15131200 & 5.94983900 \\ \mathrm{H} & 4.92644300 & -3.70989200 & -1.95820400 \\ \mathrm{H} & 2.68195300 & -3.16389600 & -2.62167400 \\ \mathrm{H} & 3.15530000 & -3.85931400 & -0.33740700 \\ \mathrm{H} & 3.77334200 & -0.16098700 & -2.92376100 \\ \mathrm{H} & 4.35253000 & 2.00914800 & -1.45917400 \\ \mathrm{H} & 4.62193100 & 1.23864100 & 1.10629900 \\ \mathrm{H} & 4.21146900 & -1.41033000 & 1.22035300 \\ \mathrm{H} & -3.44434100 & -4.95019800 & -1.07123900 \\ \mathrm{H} & -0.18428700 & 0.71295000 & -2.61641900 \\ \mathrm{H} & 1.34891100 & -1.42242700 & 0.17831100 \\ & & 0.80628800 & 0.25332600\end{array}$

$\begin{array}{cccc}\text { TS1 }^{\text {BS-CH }} & & & \\ \mathrm{Ti} & 0.31503400 & 1.18915800 & 0.48081600 \\ \mathrm{Ti} & 1.48206100 & -0.47109100 & -0.86947100 \\ \mathrm{Ti} & -1.26974000 & -0.99372500 & -0.46019100 \\ \mathrm{Si} & -0.59517600 & 4.46946000 & -0.99858500 \\ \mathrm{Si} & 4.88379200 & -0.17237200 & 0.66035000 \\ \mathrm{Si} & -4.52059900 & 0.89901900 & -0.19572100 \\ \mathrm{C} & -0.23068700 & -1.79938300 & 3.46096300 \\ \mathrm{C} & -1.34815100 & -1.22906000 & 2.90050600 \\ \mathrm{C} & -2.00276100 & -1.82305300 & 1.78159300 \\ \mathrm{C} & -1.45668000 & -3.01532000 & 1.19622500 \\ & & & \end{array}$




\begin{tabular}{|c|c|c|c|}
\hline $\mathrm{C}$ & -0.33266600 & -3.63615200 & 1.86779000 \\
\hline $\mathrm{C}$ & 0.26515800 & -3.02882200 & 2.93821600 \\
\hline $\mathrm{C}$ & -0.00326500 & 3.53242500 & 0.49926600 \\
\hline $\mathrm{C}$ & 1.36343900 & 3.28119200 & 0.85639100 \\
\hline $\mathrm{C}$ & 1.40597300 & 2.60949700 & 2.10226200 \\
\hline $\mathrm{C}$ & 0.07390000 & 2.41966900 & 2.54275800 \\
\hline $\mathrm{C}$ & -0.78859700 & 2.98176400 & 1.56190900 \\
\hline $\mathrm{C}$ & 3.85634900 & -0.74886300 & -0.78821200 \\
\hline $\mathrm{C}$ & 3.55252800 & -0.00763700 & -1.97246700 \\
\hline $\mathrm{C}$ & 2.79433800 & -0.82131900 & -2.84878600 \\
\hline $\mathrm{C}$ & 2.61785900 & -2.08291300 & -2.21480500 \\
\hline $\mathrm{C}$ & 3.26816300 & -2.04206200 & -0.95380700 \\
\hline $\mathrm{C}$ & -2.56646800 & -0.02920600 & -2.20564700 \\
\hline $\mathrm{C}$ & -2.02517900 & -1.23924700 & -2.70060500 \\
\hline $\mathrm{C}$ & -2.59936400 & -2.31019700 & -1.96222000 \\
\hline $\mathrm{C}$ & -3.49402900 & -1.74859000 & -1.02113400 \\
\hline $\mathrm{C}$ & -3.47957000 & -0.32598900 & -1.14815200 \\
\hline $\mathrm{H}$ & 0.27781800 & -0.79530800 & 0.58034700 \\
\hline $\mathrm{H}$ & 1.42514800 & 1.33296900 & -0.96656200 \\
\hline $\mathrm{H}$ & 0.12223000 & -1.64394700 & -1.36224100 \\
\hline $\mathrm{H}$ & -0.24510200 & 0.40021600 & -1.18568000 \\
\hline $\mathrm{H}$ & 0.25330100 & -1.34381700 & 4.31977400 \\
\hline $\mathrm{H}$ & -1.77848800 & -0.32708100 & 3.32783500 \\
\hline $\mathrm{H}$ & -3.03283600 & -1.54408700 & 1.59108700 \\
\hline $\mathrm{H}$ & -2.13863900 & -3.67947500 & 0.66930400 \\
\hline $\mathrm{H}$ & 0.02898400 & -4.59288200 & 1.50030000 \\
\hline $\mathrm{H}$ & -5.90504300 & 0.94554100 & -0.74416700 \\
\hline $\mathrm{H}$ & -3.93157200 & 2.25717400 & -0.29328800 \\
\hline $\mathrm{H}$ & -4.62296100 & 0.49429500 & 1.23292700 \\
\hline $\mathrm{H}$ & -2.30406000 & 0.96341000 & -2.54751500 \\
\hline $\mathrm{H}$ & -1.28983200 & -1.33113900 & -3.48831300 \\
\hline $\mathrm{H}$ & -2.39202600 & -3.36353700 & -2.10054500 \\
\hline $\mathrm{H}$ & -4.08689000 & -2.31065100 & -0.30918700 \\
\hline $\mathrm{H}$ & -1.87109300 & 2.98310100 & 1.60750400 \\
\hline $\mathrm{H}$ & -0.23288200 & 1.91954100 & 3.45305600 \\
\hline $\mathrm{H}$ & 2.29981000 & 2.26667300 & 2.60761600 \\
\hline $\mathrm{H}$ & 2.22670900 & 3.54376700 & 0.25805700 \\
\hline $\mathrm{H}$ & -1.88234300 & 3.91413900 & -1.49734800 \\
\hline $\mathrm{H}$ & 0.42823100 & 4.39458400 & -2.07408300 \\
\hline $\mathrm{H}$ & -0.82699100 & 5.91071800 & -0.68995800 \\
\hline $\mathrm{H}$ & 6.32702200 & -0.47335700 & 0.43186100 \\
\hline $\mathrm{H}$ & 4.46966700 & -0.87483800 & 1.90277900 \\
\hline $\mathrm{H}$ & 4.75776500 & 1.29741800 & 0.84188100 \\
\hline $\mathrm{H}$ & 3.30673600 & -2.85122000 & -0.23401600 \\
\hline $\mathrm{H}$ & 2.06950300 & -2.92552500 & -2.61777600 \\
\hline $\mathrm{H}$ & 2.40969500 & -0.53023900 & -3.81881100 \\
\hline
\end{tabular}




$\begin{array}{crcc}\mathrm{H} & 3.83352000 & 1.02178600 & -2.15763600 \\ \mathrm{H} & 1.11120600 & -3.50861400 & 3.42457100 \\ \mathrm{H} & -0.82165900 & -2.63328700 & -0.18253100 \\ \mathrm{H} & 1.86281800 & 0.26126600 & 0.75754300 \\ \mathrm{H} & -1.42353900 & 0.52097900 & 0.56097300\end{array}$

\begin{tabular}{|c|c|c|c|}
\hline $\mathrm{A} 2^{\mathrm{BS}-\mathrm{CH}}$ & & & \\
\hline $\mathrm{Ti}$ & 0.25437000 & 1.24276600 & 0.45741300 \\
\hline $\mathrm{Ti}$ & 1.51708300 & -0.35628300 & -0.88875900 \\
\hline $\mathrm{Ti}$ & -1.20625500 & -1.03381600 & -0.42806300 \\
\hline $\mathrm{Si}$ & -1.09538000 & 4.37243800 & -1.01110000 \\
\hline $\mathrm{Si}$ & 4.86992000 & 0.01028400 & 0.74500800 \\
\hline $\mathrm{Si}$ & -4.66730600 & 0.40275500 & 0.08698300 \\
\hline $\mathrm{C}$ & -0.05081700 & -1.84306500 & 3.38223300 \\
\hline $\mathrm{C}$ & -1.16200900 & -1.33479900 & 2.78818000 \\
\hline $\mathrm{C}$ & -1.73832100 & -1.89067400 & 1.57356900 \\
\hline $\mathrm{C}$ & -1.07043500 & -3.09873500 & 0.96919900 \\
\hline $\mathrm{C}$ & 0.09486400 & -3.64577500 & 1.73617700 \\
\hline $\mathrm{C}$ & 0.57060900 & -3.03378800 & 2.84200300 \\
\hline $\mathrm{C}$ & -0.30653400 & 3.53582800 & 0.45450700 \\
\hline $\mathrm{C}$ & 1.09953100 & 3.44041200 & 0.72198600 \\
\hline $\mathrm{C}$ & 1.29684700 & 2.80340200 & 1.97108000 \\
\hline $\mathrm{C}$ & 0.02466500 & 2.48373600 & 2.50583900 \\
\hline $\mathrm{C}$ & -0.95614100 & 2.92883800 & 1.57850600 \\
\hline $\mathrm{C}$ & 3.89919700 & -0.54486100 & -0.75151200 \\
\hline $\mathrm{C}$ & 3.59371000 & 0.23155200 & -1.91271300 \\
\hline $\mathrm{C}$ & 2.89009300 & -0.57514300 & -2.84030900 \\
\hline $\mathrm{C}$ & 2.74769500 & -1.86615100 & -2.26033600 \\
\hline $\mathrm{C}$ & 3.36604800 & -1.85080500 & -0.98158600 \\
\hline $\mathrm{C}$ & -2.68508000 & -0.07345300 & -2.04737100 \\
\hline $\mathrm{C}$ & -2.01585700 & -1.14811200 & -2.67508600 \\
\hline $\mathrm{C}$ & -2.42230800 & -2.34871100 & -2.03365000 \\
\hline $\mathrm{C}$ & -3.34914500 & -2.00380800 & -1.02083600 \\
\hline $\mathrm{C}$ & -3.51708900 & -0.58789200 & -1.00474900 \\
\hline $\mathrm{H}$ & 0.35042000 & -0.74382300 & 0.56669500 \\
\hline $\mathrm{H}$ & 1.34444600 & 1.44136300 & -0.99561800 \\
\hline $\mathrm{H}$ & 0.23438000 & -1.63554400 & -1.34065300 \\
\hline $\mathrm{H}$ & -0.26726300 & 0.41113400 & -1.18862400 \\
\hline $\mathrm{H}$ & 0.35338900 & -1.39571900 & 4.28569300 \\
\hline $\mathrm{H}$ & -1.68302200 & -0.49123800 & 3.23731000 \\
\hline $\mathrm{H}$ & -2.82601600 & -1.89311000 & 1.57821900 \\
\hline $\mathrm{H}$ & -1.77835500 & -3.88532900 & 0.67901500 \\
\hline $\mathrm{H}$ & 0.54107700 & -4.56981100 & 1.37490400 \\
\hline $\mathrm{H}$ & -6.06311300 & 0.32724400 & -0.42755800 \\
\hline $\mathrm{H}$ & -4.25063900 & 1.82711500 & 0.10179600 \\
\hline $\mathrm{H}$ & -4.67047400 & -0.13753800 & 1.47265600 \\
\hline & & $\mathrm{S} 14$ & \\
\hline
\end{tabular}




$\begin{array}{lrrc}\mathrm{H} & -2.55905800 & 0.97391200 & -2.28938900 \\ \mathrm{H} & -1.29364300 & -1.06687900 & -3.47636900 \\ \mathrm{H} & -2.07907600 & -3.34748300 & -2.27470700 \\ \mathrm{H} & -3.83447000 & -2.70376000 & -0.35168500 \\ \mathrm{H} & -2.02699600 & 2.81556600 & 1.69811500 \\ \mathrm{H} & -0.16419600 & 1.96860300 & 3.43974100 \\ \mathrm{H} & 2.25392100 & 2.56655500 & 2.41795600 \\ \mathrm{H} & 1.88741900 & 3.78272000 & 0.06299000 \\ \mathrm{H} & -2.34145500 & 3.66687400 & -1.41699700 \\ \mathrm{H} & -0.14167100 & 4.38975300 & -2.15135400 \\ \mathrm{H} & -1.46881100 & 5.78408700 & -0.70594800 \\ \mathrm{H} & 6.32574900 & -0.24676100 & 0.54574300 \\ \mathrm{H} & 4.43949500 & -0.74234400 & 1.95133800 \\ \mathrm{H} & 4.70015900 & 1.46996400 & 0.96745400 \\ \mathrm{H} & 3.41761600 & -2.68510000 & -0.29212000 \\ \mathrm{H} & 2.24505400 & -2.71311500 & -2.71099500 \\ \mathrm{H} & 2.51900300 & -0.26028900 & -3.80819100 \\ \mathrm{H} & 3.83762200 & 1.27783300 & -2.04953600 \\ \mathrm{H} & 1.41690500 & -3.46664800 & 3.37114200 \\ \mathrm{H} & -0.62458600 & -2.84005200 & -0.08806700 \\ \mathrm{H} & 1.85603900 & 0.41339000 & 0.73226000 \\ \mathrm{H} & -1.44263000 & 0.48511800 & 0.52850100\end{array}$

\section{TS1 $^{\text {CS }}$}

Ti

Ti

Ti

C

C

C

C

C

$\mathrm{H}$

$\mathrm{H}$

$\mathrm{H}$

$\mathrm{H}$

C

C

C

C

C

$\mathrm{H}$

C

C

C

C

$\begin{array}{ccc}-0.34235800 & 1.14776200 & 0.81481300 \\ -1.60664000 & -0.62481100 & -0.64491100 \\ 1.16896300 & -0.48810500 & -0.76869000 \\ 0.00933700 & 3.47732700 & 0.93738900 \\ 0.80481500 & 2.88116800 & 1.96691000 \\ -0.04862800 & 2.26293200 & 2.91400400 \\ -1.38956000 & 2.46780900 & 2.49540300 \\ -1.35575400 & 3.21242000 & 1.28877200 \\ -1.73350900 & 1.24348100 & -0.33983000 \\ -1.71910900 & 0.08039000 & 1.15007500 \\ -0.02063900 & 0.85261100 & -0.93660500 \\ 1.42976900 & 0.64300200 & 0.67955000 \\ -3.97369500 & -0.80200700 & -0.75486000 \\ -3.43880400 & -1.44307000 & -1.91611100 \\ -2.67144600 & -2.56112300 & -1.50561300 \\ -2.73114900 & -2.64344100 & -0.08653600 \\ -3.53224300 & -1.57347100 & 0.37005100 \\ -1.61812900 & 0.12299200 & -2.15748400 \\ 3.29193300 & 0.00653300 & -1.61650000 \\ 2.29633000 & 0.75841400 & -2.33278200 \\ 1.48064500 & -0.14052600 & -3.07151300 \\ 1.96624300 & -1.45693300 & -2.83458100 \\ & \mathrm{~S} 143 & \end{array}$




\begin{tabular}{|c|c|c|c|}
\hline $\mathrm{C}$ & 3.06566300 & -1.36893400 & -1.95816400 \\
\hline $\mathrm{Si}$ & 0.61465700 & 4.48483100 & -0.51471800 \\
\hline $\mathrm{Si}$ & -5.08516700 & 0.69604400 & -0.72554500 \\
\hline $\mathrm{Si}$ & 4.69095800 & 0.71737900 & -0.61303300 \\
\hline $\mathrm{H}$ & 1.53430600 & -2.36991500 & -3.22454500 \\
\hline $\mathrm{H}$ & 3.63392200 & -2.21112400 & -1.57875600 \\
\hline $\mathrm{H}$ & 5.91397100 & 0.91111700 & -1.44257800 \\
\hline $\mathrm{H}$ & 4.30536600 & 2.03560100 & -0.04695100 \\
\hline $\mathrm{H}$ & 5.05908700 & -0.20533300 & 0.49758000 \\
\hline $\mathrm{H}$ & 2.18537200 & 1.83437300 & -2.30679000 \\
\hline $\mathrm{H}$ & 0.62227200 & 0.12199400 & -3.67362000 \\
\hline $\mathrm{H}$ & -2.27868200 & 2.10418200 & 2.99391300 \\
\hline $\mathrm{H}$ & -2.22330400 & 3.51662900 & 0.71761900 \\
\hline $\mathrm{H}$ & 0.63727200 & 5.93697700 & -0.17733200 \\
\hline $\mathrm{H}$ & 2.00021200 & 4.08066300 & -0.86952500 \\
\hline $\mathrm{H}$ & -0.28260000 & 4.30077000 & -1.68361900 \\
\hline $\mathrm{H}$ & 1.88708200 & 2.87749400 & 1.99926600 \\
\hline $\mathrm{H}$ & 0.26817500 & 1.70157000 & 3.78573500 \\
\hline $\mathrm{H}$ & -2.11308100 & -3.22108700 & -2.15726600 \\
\hline $\mathrm{H}$ & -3.59033700 & -1.12364800 & -2.93791500 \\
\hline $\mathrm{H}$ & -6.52164600 & 0.29732800 & -0.78751600 \\
\hline $\mathrm{H}$ & -4.89347800 & 1.45744700 & 0.53825300 \\
\hline $\mathrm{H}$ & -4.80788400 & 1.57383500 & -1.89129800 \\
\hline $\mathrm{H}$ & -3.74976100 & -1.35076100 & 1.40712900 \\
\hline $\mathrm{H}$ & -2.21939600 & -3.36872000 & 0.53311500 \\
\hline $\mathrm{C}$ & 1.87519700 & -3.35915700 & 0.86408100 \\
\hline $\mathrm{C}$ & 0.81520800 & -4.00003900 & 1.51838500 \\
\hline $\mathrm{C}$ & 0.36975200 & -3.52703300 & 2.75101700 \\
\hline $\mathrm{C}$ & 0.97487400 & -2.40698200 & 3.33540000 \\
\hline $\mathrm{C}$ & 2.03528300 & -1.77215600 & 2.69060300 \\
\hline $\mathrm{C}$ & 2.49374300 & -2.25062900 & 1.45430600 \\
\hline $\mathrm{H}$ & 2.23671000 & -3.74614200 & -0.08417500 \\
\hline $\mathrm{H}$ & 0.34687900 & -4.86945000 & 1.06485900 \\
\hline $\mathrm{H}$ & -0.44732200 & -4.02899400 & 3.26283000 \\
\hline $\mathrm{H}$ & 0.62599300 & -2.04315700 & 4.29830200 \\
\hline $\mathrm{H}$ & 2.51897300 & -0.91278800 & 3.14667500 \\
\hline $\mathrm{H}$ & 3.35706200 & -1.78649000 & 0.98629400 \\
\hline $\mathrm{H}$ & -0.10643400 & -0.78344600 & 0.56461300 \\
\hline $\mathrm{H}$ & -0.18912600 & -1.58016500 & -1.26849300 \\
\hline
\end{tabular}

\begin{tabular}{cccc}
$\mathbf{A 2}^{\mathbf{C S}}{ }^{\mathbf{C S}^{\prime}}$ & & \\
$\mathrm{Ti}$ & -0.33115300 & 1.14572400 & 0.81273600 \\
$\mathrm{Ti}$ & -1.61406800 & -0.60604000 & -0.66100900 \\
$\mathrm{Ti}$ & 1.17404500 & -0.51933200 & -0.75843000 \\
$\mathrm{C}$ & 0.05141400 & 3.47233700 & 0.90515800 \\
$\mathrm{C}$ & 0.84116400 & 2.87938800 & 1.94065700 \\
\multicolumn{3}{c}{$\mathrm{S} 144$} \\
\end{tabular}




\begin{tabular}{|c|c|c|c|}
\hline $\mathrm{C}$ & -0.01788200 & 2.28155200 & 2.89539700 \\
\hline $\mathrm{C}$ & -1.35721200 & 2.49544400 & 2.47628400 \\
\hline $\mathrm{C}$ & -1.31664100 & 3.22581000 & 1.26077700 \\
\hline $\mathrm{H}$ & -1.73051500 & 1.25726500 & -0.34121700 \\
\hline $\mathrm{H}$ & -1.72178600 & 0.09092800 & 1.13920600 \\
\hline $\mathrm{H}$ & -0.08633200 & 0.75618200 & -0.95380000 \\
\hline $\mathrm{H}$ & 1.42959300 & 0.63711500 & 0.66699100 \\
\hline $\mathrm{C}$ & -3.98152500 & -0.76041700 & -0.74303500 \\
\hline $\mathrm{C}$ & -3.46654100 & -1.38968700 & -1.92006000 \\
\hline $\mathrm{C}$ & -2.70499300 & -2.52032500 & -1.53495600 \\
\hline $\mathrm{C}$ & -2.74898100 & -2.62282800 & -0.11657000 \\
\hline $\mathrm{C}$ & -3.53498700 & -1.55295300 & 0.36521300 \\
\hline $\mathrm{H}$ & -1.64259700 & 0.16004300 & -2.16367500 \\
\hline $\mathrm{C}$ & 3.30979300 & -0.02477100 & -1.57014100 \\
\hline $\mathrm{C}$ & 2.32534400 & 0.74843200 & -2.28076500 \\
\hline $\mathrm{C}$ & 1.51108700 & -0.13097200 & -3.04500600 \\
\hline $\mathrm{C}$ & 1.98538700 & -1.45476700 & -2.82934000 \\
\hline $\mathrm{C}$ & 3.07650000 & -1.39141900 & -1.94005500 \\
\hline $\mathrm{Si}$ & 0.66897500 & 4.45905800 & -0.55557100 \\
\hline $\mathrm{Si}$ & -5.07685000 & 0.74829500 & -0.67687000 \\
\hline $\mathrm{Si}$ & 4.70353100 & 0.65748500 & -0.54076300 \\
\hline $\mathrm{H}$ & 1.55022300 & -2.35721400 & -3.23990600 \\
\hline $\mathrm{H}$ & 3.63380200 & -2.24533400 & -1.57047100 \\
\hline $\mathrm{H}$ & 5.93361800 & 0.86876300 & -1.35566500 \\
\hline $\mathrm{H}$ & 4.31801600 & 1.96259100 & 0.05537600 \\
\hline $\mathrm{H}$ & 5.06204800 & -0.29283000 & 0.54986300 \\
\hline $\mathrm{H}$ & 2.22323100 & 1.82447100 & -2.23756600 \\
\hline $\mathrm{H}$ & 0.66079200 & 0.14908200 & -3.65079300 \\
\hline $\mathrm{H}$ & -2.24951100 & 2.14711700 & 2.97999000 \\
\hline $\mathrm{H}$ & -2.18157900 & 3.53428600 & 0.68803600 \\
\hline $\mathrm{H}$ & 0.68342900 & 5.91650800 & -0.24109000 \\
\hline $\mathrm{H}$ & 2.06001100 & 4.05290900 & -0.88576200 \\
\hline $\mathrm{H}$ & -0.21204500 & 4.25521000 & -1.73358500 \\
\hline $\mathrm{H}$ & 1.92332800 & 2.86329600 & 1.97135500 \\
\hline $\mathrm{H}$ & 0.29429100 & 1.72507900 & 3.77201800 \\
\hline $\mathrm{H}$ & -2.15969900 & -3.17528000 & -2.20250400 \\
\hline $\mathrm{H}$ & -3.62795800 & -1.05463300 & -2.93528000 \\
\hline $\mathrm{H}$ & -6.51822500 & 0.36517500 & -0.72129900 \\
\hline $\mathrm{H}$ & -4.85731500 & 1.49066000 & 0.59367900 \\
\hline $\mathrm{H}$ & -4.80889600 & 1.63886600 & -1.83511600 \\
\hline $\mathrm{H}$ & -3.73851300 & -1.34370900 & 1.40787500 \\
\hline $\mathrm{H}$ & -2.23610700 & -3.36129600 & 0.48620700 \\
\hline $\mathrm{C}$ & 1.81135600 & -3.36177600 & 0.84086300 \\
\hline $\mathrm{C}$ & 0.74067800 & -3.99548700 & 1.48616600 \\
\hline $\mathrm{C}$ & 0.29511500 & -3.52676700 & 2.71981900 \\
\hline $\mathrm{C}$ & 0.90980300 & -2.41715300 & 3.31540200 \\
\hline
\end{tabular}




$\begin{array}{lrrr}\mathrm{C} & 1.98014000 & -1.79000600 & 2.68049700 \\ \mathrm{C} & 2.44012300 & -2.26512300 & 1.44277400 \\ \mathrm{H} & 2.17593700 & -3.74906300 & -0.10603800 \\ \mathrm{H} & 0.26518800 & -4.85658900 & 1.02432500 \\ \mathrm{H} & -0.52952000 & -4.02353600 & 3.22452000 \\ \mathrm{H} & 0.56053900 & -2.05677200 & 4.27944900 \\ \mathrm{H} & 2.47202400 & -0.93991200 & 3.14512600 \\ \mathrm{H} & 3.31431300 & -1.81113100 & 0.98569300 \\ \mathrm{H} & -0.12024700 & -0.79412700 & 0.56343900 \\ \mathrm{H} & -0.20767500 & -1.58065700 & -1.28153500\end{array}$

\section{$\mathrm{C}_{6} \mathrm{H}_{6 \mathrm{D}}$}

$\mathrm{C}$

C

C

C

C

C

$\mathrm{H}$

$\mathrm{H}$

$\mathrm{H}$

$\mathrm{H}$

$\mathrm{H}$

$\mathrm{H}$

$\mathbf{1}_{\mathrm{D}}^{\mathrm{CS}}$

$\mathrm{Ti}$

$\mathrm{Ti}$

Ti

$\mathrm{Si}$

$\mathrm{Si}$

$\mathrm{Si}$

C

C

C

C

C

C

C

C

C

C

C

C

C

C

$\begin{array}{ccc}0.00000000 & 1.39649000 & 0.00000000 \\ -1.20939600 & 0.69824500 & 0.00000000 \\ -1.20939600 & -0.69824500 & 0.00000000 \\ 0.00000000 & -1.39649000 & 0.00000000 \\ 1.20939600 & -0.69824500 & 0.00000000 \\ 1.20939600 & 0.69824500 & 0.00000000 \\ 0.00000000 & 2.48319100 & 0.00000000 \\ -2.15050600 & 1.24159500 & 0.00000000 \\ -2.15050600 & -1.24159500 & 0.00000000 \\ 0.00000000 & -2.48319100 & 0.00000000 \\ 2.15050600 & -1.24159500 & 0.00000000 \\ 2.15050600 & 1.24159500 & 0.00000000\end{array}$

$\begin{array}{rrr}0.28033400 & 1.23108700 & -0.49769600\end{array}$

$\begin{array}{lll}-1.30315300 & -0.76381100 & -0.62501000\end{array}$

$\begin{array}{lll}1.03613600 & -1.05445300 & 0.35903900\end{array}$

$\begin{array}{lll}-4.15583500 & -0.81296500 & 1.69858100\end{array}$

$\begin{array}{lll}-0.45362100 & 3.85996100 & 1.97217300\end{array}$

$\begin{array}{lll}4.46577500 & -1.03173400 & -0.90724300\end{array}$

$-3.52488500 \quad-1.24924500 \quad-0.00403200$

$-2.90362700 \quad-2.47441600 \quad-0.40684000$

$-2.57052100 \quad-2.39917100 \quad-1.78410500$

$-2.98138500 \quad-1.12738600 \quad-2.26046100$

$-3.56524700 \quad-0.42547500 \quad-1.17413000$

$\begin{array}{lll}0.22757500 & 3.45548200 & 0.28111100\end{array}$

$\begin{array}{lll}1.58335000 & 3.13602800 & -0.04938200\end{array}$

$\begin{array}{lll}1.67967600 & 2.89113400 & -1.44374400\end{array}$

$\begin{array}{lll}0.38637500 & 3.05735300 & -2.00329100\end{array}$

$\begin{array}{lll}-0.50057200 & 3.40206200 & -0.95062400\end{array}$

$\begin{array}{lll}3.27548000 & -1.75541800 & 0.33922600\end{array}$

$\begin{array}{lll}2.45741400 & -2.91695700 & 0.18041400\end{array}$

$\begin{array}{lll}1.69164500 & -3.10394000 & 1.35961100\end{array}$

$\begin{array}{lll}2.02983700 & -2.06951500 & 2.27419700\end{array}$ 


\begin{tabular}{|c|c|c|c|}
\hline $\mathrm{C}$ & 2.99822300 & -1.24631400 & 1.65094400 \\
\hline $\mathrm{H}$ & 1.41901000 & 0.66269500 & 0.80577100 \\
\hline $\mathrm{H}$ & 1.56821500 & 0.02658600 & -1.01651300 \\
\hline $\mathrm{H}$ & -1.52429600 & 1.03082700 & -0.43639800 \\
\hline $\mathrm{H}$ & -0.47607500 & 0.29252600 & -1.88705500 \\
\hline $\mathrm{H}$ & -0.57358600 & -1.85123700 & 0.64647800 \\
\hline $\mathrm{H}$ & 0.19356000 & -1.72134300 & -1.12877500 \\
\hline $\mathrm{H}$ & -0.44258100 & 0.08792000 & 0.87078500 \\
\hline $\mathrm{H}$ & -5.57821700 & -1.22504800 & 1.86811100 \\
\hline $\mathrm{H}$ & -3.34543700 & -1.51766700 & 2.72549800 \\
\hline $\mathrm{H}$ & -4.07091000 & 0.65625200 & 1.89904400 \\
\hline $\mathrm{H}$ & -2.68992700 & -3.31371400 & 0.24314000 \\
\hline $\mathrm{H}$ & -2.05898300 & -3.16161000 & -2.35750000 \\
\hline $\mathrm{H}$ & -2.83943600 & -0.74434400 & -3.26286300 \\
\hline $\mathrm{H}$ & -3.94955000 & 0.58592600 & -1.21708200 \\
\hline $\mathrm{H}$ & 2.39732100 & -3.52943400 & -0.71087000 \\
\hline $\mathrm{H}$ & 0.95446400 & -3.87968200 & 1.52112700 \\
\hline $\mathrm{H}$ & 1.59665800 & -1.91735800 & 3.25515200 \\
\hline $\mathrm{H}$ & 3.43652000 & -0.35491800 & 2.08197300 \\
\hline $\mathrm{H}$ & 4.53989900 & 0.44190700 & -0.73264900 \\
\hline $\mathrm{H}$ & 5.83603200 & -1.58657400 & -0.71430100 \\
\hline $\mathrm{H}$ & 4.01824300 & -1.36631500 & -2.28257100 \\
\hline $\mathrm{H}$ & 2.57343400 & 2.59068700 & -1.97550400 \\
\hline $\mathrm{H}$ & 0.11516700 & 2.90842800 & -3.04056700 \\
\hline $\mathrm{H}$ & -1.56569600 & 3.56514900 & -1.05810300 \\
\hline $\mathrm{H}$ & 2.40116300 & 3.05813000 & 0.65599100 \\
\hline $\mathrm{H}$ & -1.87482000 & 3.43541200 & 2.05152800 \\
\hline $\mathrm{H}$ & 0.34764900 & 3.16160400 & 3.01061200 \\
\hline $\mathrm{H}$ & -0.38928100 & 5.32408500 & 2.24368600 \\
\hline
\end{tabular}

$\begin{array}{lccc}\mathbf{A 1}_{\mathbf{D}}{ }^{\mathbf{C S}^{\prime}} & & & \\ \mathrm{Ti} & 0.04062700 & 1.08111200 & 0.62009000 \\ \mathrm{Ti} & -1.62987700 & -0.48443500 & -0.50516400 \\ \mathrm{Ti} & 0.82403100 & -0.52836200 & -1.19778600 \\ \mathrm{C} & 0.88954900 & 3.24660400 & 0.93722900 \\ \mathrm{C} & 1.60232700 & 2.37346100 & 1.82319700 \\ \mathrm{C} & 0.70257100 & 1.85398100 & 2.78673300 \\ \mathrm{C} & -0.58315100 & 2.38659800 & 2.50698500 \\ \mathrm{C} & -0.46843300 & 3.24330000 & 1.38070700 \\ \mathrm{H} & -1.40693900 & 1.33288300 & -0.46847200 \\ \mathrm{H} & -1.49812500 & 0.26551900 & 1.14901000 \\ \mathrm{H} & 0.71389900 & 1.29418100 & -1.06928800 \\ \mathrm{H} & 1.59039700 & 0.20737900 & 0.27953800 \\ \mathrm{C} & -3.88903500 & 0.07697700 & -0.82482500 \\ \mathrm{C} & -3.49903400 & -0.77541600 & -1.90275200 \\ \mathrm{C} & -3.18140900 & -2.05701700 & -1.38335100 \\ & & & \end{array}$




\begin{tabular}{|c|c|c|c|}
\hline $\mathrm{C}$ & -3.38376600 & -2.02534600 & 0.02267700 \\
\hline $\mathrm{C}$ & -3.81219600 & -0.72057400 & 0.36462900 \\
\hline $\mathrm{H}$ & -0.73437000 & -0.06895500 & -2.05842700 \\
\hline $\mathrm{C}$ & 3.14327000 & -0.77789100 & -1.58498500 \\
\hline $\mathrm{C}$ & 2.57705400 & -0.08647500 & -2.70591100 \\
\hline $\mathrm{C}$ & 1.67430200 & -0.95117100 & -3.36823900 \\
\hline $\mathrm{C}$ & 1.66730700 & -2.19099300 & -2.66954500 \\
\hline $\mathrm{C}$ & 2.57139600 & -2.08828900 & -1.58396700 \\
\hline $\mathrm{Si}$ & 1.58873300 & 4.19112800 & -0.51593900 \\
\hline $\mathrm{Si}$ & -4.38145800 & 1.87634100 & -0.92906000 \\
\hline $\mathrm{Si}$ & 4.38901500 & -0.09664600 & -0.37163500 \\
\hline $\mathrm{H}$ & 1.05458300 & -3.05049200 & -2.90863600 \\
\hline $\mathrm{H}$ & 2.75860800 & -2.85773800 & -0.84633400 \\
\hline $\mathrm{H}$ & 5.78070700 & -0.34580900 & -0.84400800 \\
\hline $\mathrm{H}$ & 4.20772500 & 1.36828900 & -0.21955500 \\
\hline $\mathrm{H}$ & 4.22978200 & -0.76468500 & 0.94601700 \\
\hline $\mathrm{H}$ & 2.77598700 & 0.94364200 & -2.97495200 \\
\hline $\mathrm{H}$ & 1.06553100 & -0.70167000 & -4.22772200 \\
\hline $\mathrm{H}$ & -1.49842200 & 2.15777700 & 3.03731700 \\
\hline $\mathrm{H}$ & -1.28870800 & 3.77086200 & 0.90965300 \\
\hline $\mathrm{H}$ & 2.05799800 & 5.54111000 & -0.09014700 \\
\hline $\mathrm{H}$ & 2.74962700 & 3.46078500 & -1.08427800 \\
\hline $\mathrm{H}$ & 0.53636500 & 4.37162800 & -1.54752900 \\
\hline $\mathrm{H}$ & 2.65400800 & 2.12793500 & 1.74651000 \\
\hline $\mathrm{H}$ & 0.94386700 & 1.14352600 & 3.56603900 \\
\hline $\mathrm{H}$ & -2.81965000 & -2.90129100 & -1.95582800 \\
\hline $\mathrm{H}$ & -3.41367000 & -0.48036000 & -2.94135400 \\
\hline $\mathrm{H}$ & -5.85582400 & 2.01311300 & -1.10648100 \\
\hline $\mathrm{H}$ & -4.00772800 & 2.57140700 & 0.33013800 \\
\hline $\mathrm{H}$ & -3.71329400 & 2.51719000 & -2.08924800 \\
\hline $\mathrm{H}$ & -4.01920100 & -0.36907200 & 1.36778600 \\
\hline $\mathrm{H}$ & -3.19955900 & -2.83783100 & 0.71273100 \\
\hline $\mathrm{C}$ & 0.88231400 & -3.58932800 & 1.40482200 \\
\hline $\mathrm{C}$ & -0.46264000 & -3.54104000 & 1.78211600 \\
\hline $\mathrm{C}$ & -0.88440200 & -2.62601100 & 2.74772800 \\
\hline $\mathrm{C}$ & 0.03867400 & -1.75800600 & 3.33790200 \\
\hline $\mathrm{C}$ & 1.38151300 & -1.80641000 & 2.96089900 \\
\hline $\mathrm{C}$ & 1.80369600 & -2.72307400 & 1.99421100 \\
\hline $\mathrm{H}$ & 1.20522500 & -4.29303900 & 0.64203800 \\
\hline $\mathrm{H}$ & -1.17983300 & -4.20845400 & 1.31175700 \\
\hline $\mathrm{H}$ & -1.93257300 & -2.57547200 & 3.03052500 \\
\hline $\mathrm{H}$ & -0.29507100 & -1.03559400 & 4.07795600 \\
\hline $\mathrm{H}$ & 2.09960500 & -1.12215100 & 3.40472000 \\
\hline $\mathrm{H}$ & 2.84691500 & -2.74524500 & 1.69267900 \\
\hline $\mathrm{H}$ & -0.02949400 & -0.83854800 & 0.48389600 \\
\hline $\mathrm{H}$ & -0.51107200 & -1.76855900 & -1.15780000 \\
\hline
\end{tabular}




\begin{tabular}{|c|c|c|c|}
\hline TS1 & & & \\
\hline $\mathrm{Ti}$ & -0.18817500 & 1.11297100 & 0.71414500 \\
\hline $\mathrm{Ti}$ & -1.59718700 & -0.61042500 & -0.63302500 \\
\hline $\mathrm{Ti}$ & 1.14862900 & -0.82462300 & -0.59052600 \\
\hline $\mathrm{C}$ & 0.41542400 & 3.36314800 & 0.55950800 \\
\hline $\mathrm{C}$ & 1.10316800 & 2.81985300 & 1.69205900 \\
\hline $\mathrm{C}$ & 0.15195000 & 2.43051000 & 2.66812600 \\
\hline $\mathrm{C}$ & -1.14119000 & 2.73045100 & 2.16262200 \\
\hline $\mathrm{C}$ & -0.98046800 & 3.30777400 & 0.87819100 \\
\hline $\mathrm{H}$ & -1.45977600 & 1.25085000 & -0.55284100 \\
\hline $\mathrm{H}$ & -1.70503800 & 0.26877400 & 1.07269000 \\
\hline $\mathrm{H}$ & 0.36047900 & 0.76929300 & -0.94269600 \\
\hline $\mathrm{H}$ & 1.50878400 & 0.43906000 & 0.70916400 \\
\hline $\mathrm{C}$ & -3.93916800 & -0.40135800 & -0.77739200 \\
\hline $\mathrm{C}$ & -3.51418200 & -1.16261300 & -1.91005500 \\
\hline $\mathrm{C}$ & -2.94181700 & -2.37520900 & -1.45202400 \\
\hline $\mathrm{C}$ & -3.03119400 & -2.39988200 & -0.03201200 \\
\hline $\mathrm{C}$ & -3.64598600 & -1.19867000 & 0.37909100 \\
\hline $\mathrm{H}$ & -1.39816800 & -0.01139600 & -2.19517900 \\
\hline $\mathrm{C}$ & 3.31811300 & -0.47434900 & -1.42996800 \\
\hline $\mathrm{C}$ & 2.36437600 & 0.13030600 & -2.31576100 \\
\hline $\mathrm{C}$ & 1.55797500 & -0.88119300 & -2.89488700 \\
\hline $\mathrm{C}$ & 2.01186100 & -2.13458200 & -2.38463300 \\
\hline $\mathrm{C}$ & 3.08656500 & -1.88391900 & -1.50623800 \\
\hline $\mathrm{Si}$ & 1.17414900 & 4.00924600 & -1.01903800 \\
\hline $\mathrm{Si}$ & -4.68871000 & 1.30581600 & -0.78981500 \\
\hline $\mathrm{Si}$ & 4.60965600 & 0.40410900 & -0.41483100 \\
\hline $\mathrm{H}$ & 1.57954300 & -3.10188400 & -2.60589900 \\
\hline $\mathrm{H}$ & 3.62104400 & -2.63570800 & -0.93640500 \\
\hline $\mathrm{H}$ & 5.89655800 & 0.53763400 & -1.15349100 \\
\hline $\mathrm{H}$ & 4.14099200 & 1.76207800 & -0.04321000 \\
\hline $\mathrm{H}$ & 4.88489100 & -0.37958400 & 0.82221600 \\
\hline $\mathrm{H}$ & 2.25708800 & 1.19273000 & -2.48771300 \\
\hline $\mathrm{H}$ & 0.72337200 & -0.72754400 & -3.56310900 \\
\hline $\mathrm{H}$ & -2.08408100 & 2.52757100 & 2.65306400 \\
\hline $\mathrm{H}$ & -1.78854300 & 3.61730100 & 0.22831200 \\
\hline $\mathrm{H}$ & 1.38869600 & 5.48279300 & -0.94657800 \\
\hline $\mathrm{H}$ & 2.49392200 & 3.36381500 & -1.23345600 \\
\hline $\mathrm{H}$ & 0.27593600 & 3.72560900 & -2.16615300 \\
\hline $\mathrm{H}$ & 2.17502000 & 2.68824800 & 1.76925100 \\
\hline $\mathrm{H}$ & 0.37044900 & 1.95090000 & 3.61505300 \\
\hline $\mathrm{H}$ & -2.48317400 & -3.13436700 & -2.07269200 \\
\hline $\mathrm{H}$ & -3.59065800 & -0.85274000 & -2.94280000 \\
\hline $\mathrm{H}$ & -6.17897100 & 1.24769700 & -0.82853900 \\
\hline $\mathrm{H}$ & -4.30271800 & 2.03489600 & 0.44827600 \\
\hline
\end{tabular}




$\begin{array}{lrrr}\mathrm{H} & -4.22633000 & 2.05892400 & -1.98315600 \\ \mathrm{H} & -3.82552200 & -0.90366800 & 1.40506000 \\ \mathrm{H} & -2.64802600 & -3.17451600 & 0.61898800 \\ \mathrm{C} & 1.34578600 & -3.10862800 & 1.14479900 \\ \mathrm{C} & 0.09648900 & -3.47828800 & 1.68174100 \\ \mathrm{C} & -0.42714400 & -2.79427700 & 2.76783600 \\ \mathrm{C} & 0.27705300 & -1.70799600 & 3.31965500 \\ \mathrm{C} & 1.52177000 & -1.35277100 & 2.81212100 \\ \mathrm{C} & 2.07850500 & -2.06522400 & 1.72896800 \\ \mathrm{H} & 1.76995400 & -3.68634300 & 0.32960900 \\ \mathrm{H} & -0.45418100 & -4.29776000 & 1.22934200 \\ \mathrm{H} & -1.39445800 & -3.07412600 & 3.17557300 \\ \mathrm{H} & -0.14672100 & -1.15583200 & 4.15399700 \\ \mathrm{H} & 2.07453100 & -0.52347200 & 3.24333600 \\ \mathrm{H} & 3.08718000 & -1.83947900 & 1.39950900 \\ \mathrm{H} & -0.19700300 & -0.78945400 & 0.65075000 \\ \mathrm{H} & -0.29559800 & -1.79150200 & -1.08224500\end{array}$

\begin{tabular}{|c|c|c|c|}
\hline \multicolumn{4}{|c|}{$\mathbf{A} 2_{\mathrm{D}}{ }^{\mathrm{CS}^{\prime}}$} \\
\hline $\mathrm{Ti}$ & -0.18908600 & 1.09721700 & 0.66210300 \\
\hline $\mathrm{Ti}$ & -1.57829100 & -0.63011100 & -0.70281000 \\
\hline $\mathrm{Ti}$ & 1.18337300 & -0.93996500 & -0.52846300 \\
\hline $\mathrm{C}$ & 0.40430200 & 3.31755600 & 0.30966600 \\
\hline $\mathrm{C}$ & 1.11113000 & 2.87181800 & 1.47434400 \\
\hline $\mathrm{C}$ & 0.17671900 & 2.56531800 & 2.49421200 \\
\hline $\mathrm{C}$ & -1.12548100 & 2.81720000 & 1.98791500 \\
\hline $\mathrm{C}$ & -0.98663400 & 3.28872900 & 0.65760200 \\
\hline $\mathrm{H}$ & -1.50735900 & 1.21448700 & -0.57793200 \\
\hline $\mathrm{H}$ & -1.69460400 & 0.23179000 & 1.03812200 \\
\hline $\mathrm{H}$ & 0.08810500 & 0.42453000 & -1.05246400 \\
\hline $\mathrm{H}$ & 1.50351000 & 0.51912800 & 0.52671500 \\
\hline $\mathrm{C}$ & -3.91806100 & -0.44354400 & -0.74796800 \\
\hline $\mathrm{C}$ & -3.53044900 & -1.13307900 & -1.93892500 \\
\hline $\mathrm{C}$ & -2.92993800 & -2.36259500 & -1.57523700 \\
\hline $\mathrm{C}$ & -2.96772100 & -2.47244900 & -0.15679400 \\
\hline $\mathrm{C}$ & -3.57729200 & -1.30622800 & 0.34835800 \\
\hline $\mathrm{H}$ & -1.44662300 & 0.01255700 & -2.25256500 \\
\hline $\mathrm{C}$ & 3.39737600 & -0.57476200 & -1.25708300 \\
\hline $\mathrm{C}$ & 2.46876200 & -0.04940800 & -2.21802200 \\
\hline $\mathrm{C}$ & 1.72215200 & -1.11930800 & -2.77488900 \\
\hline $\mathrm{C}$ & 2.18242200 & -2.32660600 & -2.16691700 \\
\hline $\mathrm{C}$ & 3.20631100 & -1.99033600 & -1.25491800 \\
\hline $\mathrm{Si}$ & 1.13780400 & 3.83489100 & -1.32520300 \\
\hline $\mathrm{Si}$ & -4.68296000 & 1.25282100 & -0.62737200 \\
\hline $\mathrm{Si}$ & 4.61612300 & 0.40694100 & -0.24673800 \\
\hline $\mathrm{H}$ & 1.78963600 & -3.31889500 & -2.34873300 \\
\hline
\end{tabular}




$\begin{array}{lrrr}\mathrm{H} & 3.72689700 & -2.69104500 & -0.61148900 \\ \mathrm{H} & 5.94556700 & 0.48751700 & -0.91412300 \\ \mathrm{H} & 4.11643400 & 1.78780000 & -0.03413900 \\ \mathrm{H} & 4.82135100 & -0.25119300 & 1.07427200 \\ \mathrm{H} & 2.33833800 & 0.99734700 & -2.45591500 \\ \mathrm{H} & 0.92288400 & -1.03070800 & -3.49674800 \\ \mathrm{H} & -2.05940200 & 2.65229700 & 2.50873100 \\ \mathrm{H} & -1.80596100 & 3.55004400 & 0.00096900 \\ \mathrm{H} & 1.26873500 & 5.31789500 & -1.40983900 \\ \mathrm{H} & 2.49471100 & 3.24705300 & -1.45961500 \\ \mathrm{H} & 0.27353300 & 3.38115300 & -2.44309400 \\ \mathrm{H} & 2.18451300 & 2.74993500 & 1.54492400 \\ \mathrm{H} & 0.41141400 & 2.16439500 & 3.47333000 \\ \mathrm{H} & -2.48419400 & -3.07715500 & -2.25533300 \\ \mathrm{H} & -3.64952200 & -0.76476400 & -2.94811700 \\ \mathrm{H} & -6.17288400 & 1.18142000 & -0.63081800 \\ \mathrm{H} & -4.26779100 & 1.90111500 & 0.64529500 \\ \mathrm{H} & -4.25969200 & 2.08929300 & -1.77918400 \\ \mathrm{H} & -3.72136500 & -1.07483500 & 1.39575800 \\ \mathrm{H} & -2.55141800 & -3.27932900 & 0.43074800 \\ \mathrm{C} & 1.25641500 & -2.93188500 & 1.36414900 \\ \mathrm{C} & -0.01882200 & -3.24031400 & 1.89762500 \\ \mathrm{C} & -0.59591000 & -2.43064800 & 2.85770500 \\ \mathrm{C} & -0.227653600 & -1.26497800 & 3.29243300 \\ \mathrm{C} & 1.34730900 & -0.97643300 & 2.81093700 \\ \mathrm{C} & 1.96594200 & -1.82176500 & 1.85629600 \\ \mathrm{H} & 1.73249300 & -3.62935200 & 0.68224700 \\ \mathrm{H} & -0.53947900 & -4.12277800 & 1.53721100 \\ \mathrm{H} & -1.57886500 & -2.66644900 & 3.25489800 \\ \mathrm{H} & -0.38750100 & -0.61264500 & 4.02662300 \\ \mathrm{H} & 1.88389300 & -0.10244800 & 3.16800400 \\ \mathrm{H} & 3.00633100 & -1.66162500 & 1.59674900 \\ \mathrm{H} & -0.80885300 & 0.61945400 \\ \mathrm{H} & -1.83763200 & -1.10473700\end{array}$

$\begin{array}{lccc}\mathbf{D - A 1}^{\mathbf{C S}} & & & \\ \mathrm{Ti} & -0.82786900 & -0.38718900 & -1.05519400 \\ \mathrm{Ti} & 0.74531800 & -0.08985200 & 0.96494500 \\ \mathrm{Si} & 2.82140900 & 4.23587100 & 0.74457800 \\ \mathrm{Si} & -0.36174300 & -4.07914800 & -1.56274100 \\ \mathrm{Si} & -0.94932300 & -1.89120900 & 3.74093900 \\ \mathrm{C} & 1.25433500 & 4.02645200 & -0.25170900 \\ \mathrm{C} & -0.09057200 & 4.18627100 & 0.21710500 \\ \mathrm{C} & -0.97863200 & 4.01252700 & -0.87104000 \\ \mathrm{C} & -0.19892600 & 3.74398400 & -2.03018700 \\ \mathrm{C} & 1.16700800 & 3.75603300 & -1.65367700 \\ & & \mathrm{~S}\end{array}$




\begin{tabular}{|c|c|c|c|}
\hline $\mathrm{C}$ & -1.34761900 & -2.53830900 & -1.94306500 \\
\hline $\mathrm{C}$ & -2.49790100 & -2.05378000 & -1.23575200 \\
\hline $\mathrm{C}$ & -2.99487300 & -0.90445400 & -1.89513200 \\
\hline $\mathrm{C}$ & -2.15636500 & -0.65396300 & -3.01553100 \\
\hline $\mathrm{C}$ & -1.15127600 & -1.65544700 & -3.04883400 \\
\hline $\mathrm{C}$ & 0.65349000 & -1.26009700 & 3.01909400 \\
\hline $\mathrm{C}$ & 1.26765300 & 0.00623000 & 3.26777500 \\
\hline $\mathrm{C}$ & 2.50449600 & 0.06221500 & 2.57254800 \\
\hline $\mathrm{C}$ & 2.68047100 & -1.16588800 & 1.88278000 \\
\hline $\mathrm{C}$ & 1.54719900 & -1.97316500 & 2.15342000 \\
\hline $\mathrm{H}$ & -0.38971800 & 1.09163300 & -2.02820600 \\
\hline $\mathrm{H}$ & -1.55179700 & 1.20522700 & -0.48470300 \\
\hline $\mathrm{H}$ & -0.19238600 & 1.47142600 & 1.24843700 \\
\hline $\mathrm{H}$ & -1.01644100 & -0.55393400 & 0.77053600 \\
\hline $\mathrm{H}$ & 0.39371700 & -1.44741300 & -0.20616100 \\
\hline $\mathrm{H}$ & 3.18242400 & 0.90586400 & 2.55398900 \\
\hline $\mathrm{H}$ & 0.84838200 & 0.80427800 & 3.86859300 \\
\hline $\mathrm{H}$ & 3.51071700 & -1.42440400 & 1.23700200 \\
\hline $\mathrm{H}$ & 1.37258600 & -2.96474800 & 1.75421300 \\
\hline $\mathrm{H}$ & -1.55203800 & -2.91274000 & 2.84641400 \\
\hline $\mathrm{H}$ & -0.71368200 & -2.53153900 & 5.06694300 \\
\hline $\mathrm{H}$ & -1.90407400 & -0.77046700 & 3.94687200 \\
\hline $\mathrm{H}$ & -2.05978000 & 4.04768400 & -0.82565900 \\
\hline $\mathrm{H}$ & -0.38026600 & 4.38394400 & 1.24204600 \\
\hline $\mathrm{H}$ & -0.58381000 & 3.54596000 & -3.02237500 \\
\hline $\mathrm{H}$ & 2.00551700 & 3.57061100 & -2.31503200 \\
\hline $\mathrm{H}$ & 3.24201600 & 5.66595800 & 0.76106200 \\
\hline $\mathrm{H}$ & 2.61215100 & 3.80949400 & 2.15245000 \\
\hline $\mathrm{H}$ & 3.92360200 & 3.44641800 & 0.13518700 \\
\hline $\mathrm{H}$ & 1.03613500 & -3.92508400 & -2.04527500 \\
\hline $\mathrm{H}$ & -0.36468100 & -4.35995200 & -0.10417000 \\
\hline $\mathrm{H}$ & -0.95124000 & -5.26074000 & -2.25489100 \\
\hline $\mathrm{H}$ & -2.90899500 & -2.48337200 & -0.33063100 \\
\hline $\mathrm{H}$ & -3.83944000 & -0.30622300 & -1.57707800 \\
\hline $\mathrm{H}$ & -2.25495700 & 0.16992600 & -3.71089700 \\
\hline $\mathrm{H}$ & -0.35457100 & -1.72512500 & -3.78066700 \\
\hline $\mathrm{C}$ & 5.45717400 & -2.87189500 & -0.78236100 \\
\hline $\mathrm{C}$ & 6.36916500 & -2.03855200 & -0.12885700 \\
\hline $\mathrm{C}$ & 6.27512700 & -0.65155000 & -0.27205200 \\
\hline $\mathrm{C}$ & 5.26815500 & -0.09769800 & -1.06702000 \\
\hline $\mathrm{C}$ & 4.35440600 & -0.93080900 & -1.71863800 \\
\hline $\mathrm{C}$ & 4.45001600 & -2.31810200 & -1.57732500 \\
\hline $\mathrm{H}$ & 5.53299200 & -3.95085800 & -0.67427200 \\
\hline $\mathrm{H}$ & 7.15374900 & -2.46926800 & 0.48787200 \\
\hline $\mathrm{H}$ & 6.98711600 & -0.00379500 & 0.23303000 \\
\hline $\mathrm{H}$ & 5.19221700 & 0.98116900 & -1.17581400 \\
\hline
\end{tabular}




$\begin{array}{cccc}\mathrm{H} & 3.56170000 & -0.49971300 & -2.32384200 \\ \mathrm{H} & 3.73781200 & -2.96504400 & -2.08302600 \\ \mathrm{Ti} & 0.13217600 & 1.93803400 & -0.50076900 \\ \mathrm{H} & 0.98513800 & 0.25561900 & -0.92915400 \\ \mathrm{H} & 1.59319700 & 1.45400400 & 0.48070100 \\ \mathrm{C} & -4.97849000 & 0.04202400 & 1.58068300 \\ \mathrm{C} & -5.67545800 & -1.11371900 & 1.21844700 \\ \mathrm{C} & -6.62546900 & -1.06609400 & 0.19455800 \\ \mathrm{C} & -6.88077700 & 0.13842500 & -0.46600200 \\ \mathrm{C} & -6.18655300 & 1.29558900 & -0.10163800 \\ \mathrm{C} & -5.23500900 & 1.24690400 & 0.92063500 \\ \mathrm{H} & -4.23550400 & 0.00536100 & 2.37269300 \\ \mathrm{H} & -5.48230200 & -2.04939500 & 1.73724300 \\ \mathrm{H} & -7.16900800 & -1.96486100 & -0.08515900 \\ \mathrm{H} & -7.62350900 & 0.17662900 & -1.25874100 \\ \mathrm{H} & -6.39083100 & 2.23446000 & -0.61025800 \\ \mathrm{H} & -4.69334200 & 2.14516900 & 1.20491900\end{array}$

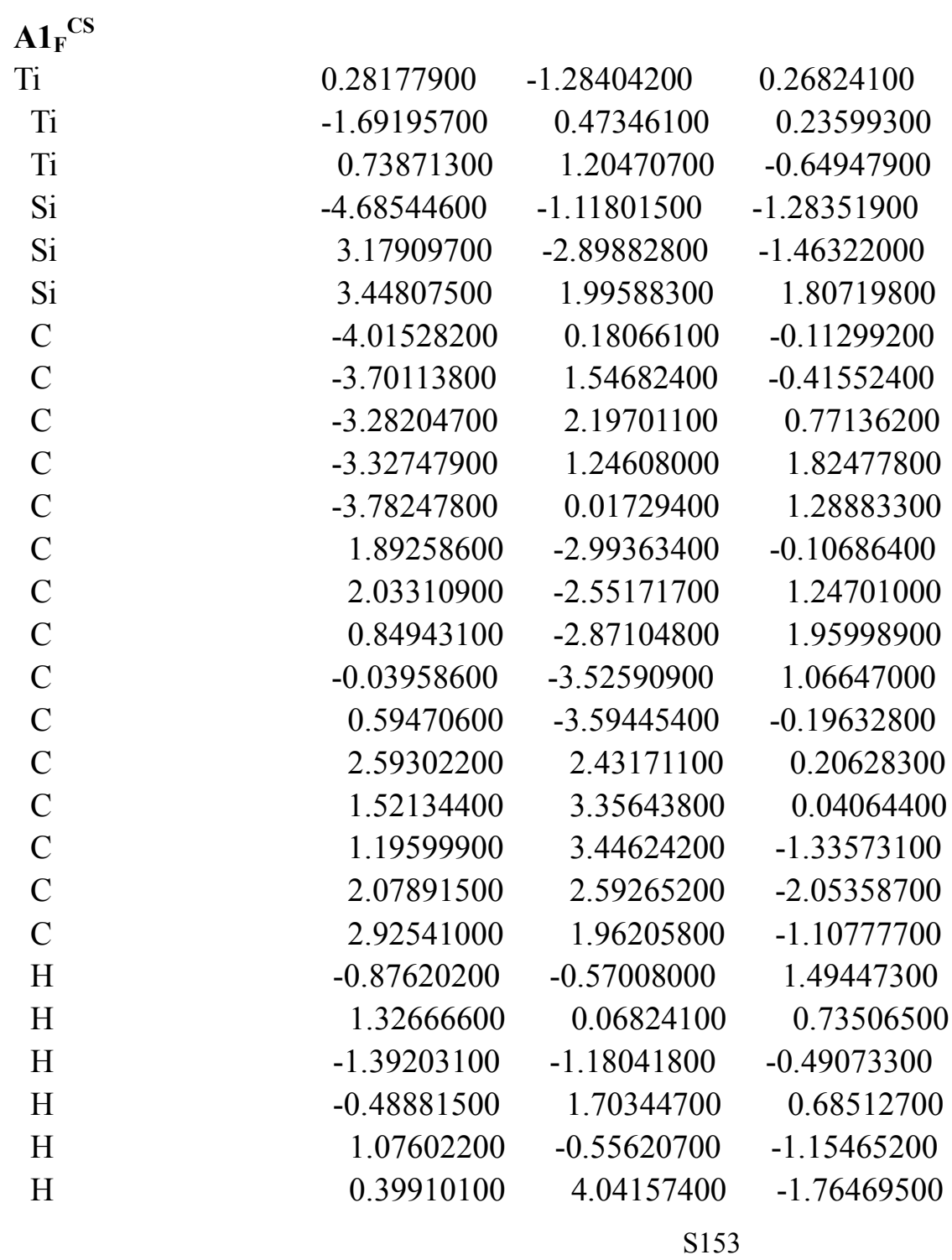




$\begin{array}{lrrr}\mathrm{H} & 1.01081100 & 3.87664800 & 0.84183600 \\ \mathrm{H} & 2.10137900 & 2.45400700 & -3.12521400 \\ \mathrm{H} & 3.68901000 & 1.22841600 & -1.33825700 \\ \mathrm{H} & 4.07509300 & 0.65137300 & 1.71236400 \\ \mathrm{H} & 4.53004400 & 2.97661200 & 2.10997600 \\ \mathrm{H} & 2.48580300 & 2.02415100 & 2.93982800 \\ \mathrm{H} & -2.95649600 & 3.22592500 & 0.85422500 \\ \mathrm{H} & -3.76045900 & 2.00599600 & -1.39483900 \\ \mathrm{H} & -3.03009400 & 1.42022300 & 2.85239300 \\ \mathrm{H} & -3.89944400 & -0.90645400 & 1.84284900 \\ \mathrm{H} & -6.14720900 & -0.92494600 & -1.49505300 \\ \mathrm{H} & -4.01507100 & -1.03068600 & -2.60622200 \\ \mathrm{H} & -4.47235700 & -2.46492100 & -0.69329700 \\ \mathrm{H} & 2.51411900 & -2.84050900 & -2.78961200 \\ \mathrm{H} & 4.04794400 & -1.71055200 & -1.26765000 \\ \mathrm{H} & 4.04753000 & -4.11022000 & -1.43688700 \\ \mathrm{H} & 2.89521400 & -2.03909000 & 1.65535300 \\ \mathrm{H} & 0.64859900 & -2.63611000 & 2.99841500 \\ \mathrm{H} & -1.03909600 & -3.87224000 & 1.29855800 \\ \mathrm{H} & 0.15597800 & -4.01057400 & -1.09692200 \\ \mathrm{H} & -1.03986300 & 1.29752400 & -1.20723400 \\ \mathrm{H} & 0.48280600 & 0.86029300 & -2.26961200\end{array}$

\section{TS1 $_{\text {F }}{ }^{\text {CS }}$}

Ti

$\mathrm{Ti}$

Ti

$\mathrm{Si}$

$\mathrm{Si}$

$\mathrm{Si}$

C

C

C

C

C

C

C

C

C

C

C

C

C

C

C

$\mathrm{H}$

$\begin{array}{ccc}0.09498700 & 1.34219800 & -0.41129400 \\ 1.39359100 & -0.82234400 & -0.52419400 \\ -1.40638200 & -1.00329300 & -1.08714100 \\ 4.94803000 & 0.00161100 & -0.79779600 \\ -2.20737300 & 4.14728200 & -0.57523800 \\ -2.48643000 & -1.69779400 & 2.45002500 \\ 3.62157500 & -0.98037700 & 0.07912500 \\ 3.16458100 & -2.30471600 & -0.23448400 \\ 2.19691300 & -2.70055700 & 0.72001200 \\ 2.02364700 & -1.62915600 & 1.63131600 \\ 2.89529100 & -0.57758100 & 1.25173000 \\ -0.75877600 & 3.36115900 & 0.31146900 \\ -0.82766600 & 2.44239100 & 1.41097900 \\ 0.49176700 & 2.10236000 & 1.81066300 \\ 1.39581600 & 2.81028000 & 0.98038100 \\ 0.63813900 & 3.57706300 & 0.06433500 \\ -2.80870100 & -1.88359200 & 0.62612900 \\ -2.38778100 & -2.95986600 & -0.21487300 \\ -2.90286000 & -2.75692700 & -1.52013900 \\ -3.65760600 & -1.54532300 & -1.50410400 \\ -3.59831000 & -1.01761200 & -0.19095900 \\ 1.78643700 & 0.91427900 & -1.05080400 \\ & \mathrm{~S} 154 & \end{array}$




$\begin{array}{lrrr}\mathrm{H} & -0.15802400 & -0.33177400 & 0.39677400 \\ \mathrm{H} & 0.15713600 & -0.02949800 & -1.68866900 \\ \mathrm{H} & 0.16384500 & -2.05019000 & -0.97388500 \\ \mathrm{H} & -1.58682500 & 0.85791900 & -0.81645000 \\ \mathrm{H} & -2.75505700 & -3.40470400 & -2.37280500 \\ \mathrm{H} & -1.75034500 & -3.78156700 & 0.08803900 \\ \mathrm{H} & -4.18009600 & -1.10880300 & -2.34381000 \\ \mathrm{H} & -4.04686600 & -0.08557900 & 0.13099600 \\ \mathrm{H} & -2.48029400 & -0.26123500 & 2.83928600 \\ \mathrm{H} & -3.53896900 & -2.37412300 & 3.26000800 \\ \mathrm{H} & -1.18049000 & -2.30665000 & 2.82481000 \\ \mathrm{H} & 1.64573500 & -3.63150500 & 0.71928900 \\ \mathrm{H} & 3.48748500 & -2.90141800 & -1.08122400 \\ \mathrm{H} & 1.31599800 & -1.60763800 & 2.45035800 \\ \mathrm{H} & 3.00208500 & 0.36888800 & 1.76333000 \\ \mathrm{H} & 6.30485300 & -0.48649900 & -0.42376200 \\ \mathrm{H} & 4.80982600 & -0.13205700 & -2.27279500 \\ \mathrm{H} & 4.84043900 & 1.43138500 & -0.40884100 \\ \mathrm{H} & -1.97783400 & 4.14594900 & -2.04452600 \\ \mathrm{H} & -3.44824200 & 3.40269000 & -0.24871200 \\ \mathrm{H} & -2.37206900 & 5.56377000 & -0.14387600 \\ \mathrm{H} & -1.73694800 & 2.04399900 & 1.84227200 \\ \mathrm{H} & 0.75633700 & 1.41246100 & 2.60100100 \\ \mathrm{H} & 2.47543700 & 2.74063700 & 1.00376200 \\ \mathrm{H} & 1.05278000 & 4.20108300 & -0.72060100 \\ \mathrm{H} & -1.07618200 & -1.54961700 & -2.64767000 \\ \mathrm{H} & -1.79381400 & -0.34520100 & -2.59100700\end{array}$

$\begin{array}{cccc}\mathbf{A 2}_{\mathbf{F}}{ }^{C S} & & & \\ \mathrm{Ti} & -0.36926100 & 1.31221800 & -0.36536600 \\ \mathrm{Ti} & 1.59229100 & -0.42021400 & -0.57107300 \\ \mathrm{Ti} & -0.93644300 & -1.26400600 & -1.10611000 \\ \mathrm{Si} & 4.78430200 & 1.26697800 & -0.52215200 \\ \mathrm{Si} & -3.02515900 & 3.65765800 & -0.97852200 \\ \mathrm{Si} & -2.15392700 & -1.87299800 & 2.44798100 \\ \mathrm{C} & 3.73719800 & -0.11927900 & 0.15583700 \\ \mathrm{C} & 3.64056400 & -1.46632900 & -0.34364800 \\ \mathrm{C} & 2.78045900 & -2.21070000 & 0.50065200 \\ \mathrm{C} & 2.31934000 & -1.34847600 & 1.52323600 \\ \mathrm{C} & 2.90121800 & -0.07154500 & 1.32523200 \\ \mathrm{C} & -1.72999900 & 3.04670900 & 0.21735900 \\ \mathrm{C} & -1.90885400 & 2.04561100 & 1.23235500 \\ \mathrm{C} & -0.69532400 & 1.89675800 & 1.94705400 \\ \mathrm{C} & 0.25245500 & 2.79782700 & 1.40677900 \\ \mathrm{C} & -0.36999700 & 3.50320400 & 0.34577900 \\ \mathrm{C} & -2.09696100 & -2.37517400 & 0.65560400 \\ & & & \end{array}$




$\begin{array}{cccc}\mathrm{C} & -1.15661300 & -3.26531600 & 0.03934000 \\ \mathrm{C} & -1.55568200 & -3.48963400 & -1.30826000 \\ \mathrm{C} & -2.75071100 & -2.74345300 & -1.53166100 \\ \mathrm{C} & -3.07455700 & -2.06504600 & -0.33678900 \\ \mathrm{H} & 1.44567200 & 1.42423100 & -0.78105700 \\ \mathrm{H} & -0.04881400 & -0.40220000 & 0.32268200 \\ \mathrm{H} & 0.20528900 & 0.14531800 & -1.73619800 \\ \mathrm{H} & 0.79397300 & -1.80578000 & -1.50097500 \\ \mathrm{H} & -1.77642800 & 0.39773000 & -1.15557700 \\ \mathrm{H} & -1.05034700 & -4.12123200 & -2.02742800 \\ \mathrm{H} & -0.29294100 & -3.70423900 & 0.52101300 \\ \mathrm{H} & -3.30064900 & -2.68265100 & -2.46306000 \\ \mathrm{H} & -3.90521200 & -1.38155100 & -0.20947900 \\ \mathrm{H} & -2.99573000 & -0.65973900 & 2.61763000 \\ \mathrm{H} & -2.75302300 & -2.95672100 & 3.27795400 \\ \mathrm{H} & -0.79464400 & -1.60880800 & 2.99105200 \\ \mathrm{H} & 2.48167600 & -3.24026300 & 0.35240700 \\ \mathrm{H} & 4.13613600 & -1.85110500 & -1.22851100 \\ \mathrm{H} & 1.60482600 & -1.60655600 & 2.29436600 \\ \mathrm{H} & 2.73587000 & 0.79834600 & 1.94638700 \\ \mathrm{H} & 6.22065800 & 1.08720400 & -0.16484800 \\ \mathrm{H} & 4.70166400 & 1.30830500 & -2.00852000 \\ \mathrm{H} & 4.32275700 & 2.56213400 & 0.04036200 \\ \mathrm{H} & -2.45577900 & 3.77986800 & -2.34974200 \\ \mathrm{H} & -4.17046700 & 2.71247500 & -0.99701200 \\ \mathrm{H} & -3.52907200 & 5.00524600 & -0.58871400 \\ \mathrm{H} & -2.81300400 & 1.47700200 & 1.40208100 \\ \mathrm{H} & -0.51005400 & 1.18107000 & 2.73785000 \\ \mathrm{H} & 1.28433600 & 2.90058100 & 1.71512900 \\ \mathrm{H} & 0.10865800 & 4.25545900 & -0.27141000 \\ \mathrm{H} & -0.79162200 & -1.43901400 & -3.05076000 \\ & -1.38687000 & -0.92494100 & -2.98504200\end{array}$

\begin{tabular}{cccc}
$\mathbf{A 1} \mathbf{~}_{\mathbf{F}} \mathbf{A F}^{-}$ & & & \\
$\mathrm{Ti}$ & 0.80783000 & -1.31321300 & 0.07407800 \\
$\mathrm{Ti}$ & -1.71004200 & 0.02499000 & 0.31852900 \\
$\mathrm{Ti}$ & 0.28761200 & 1.36311900 & -0.76319400 \\
$\mathrm{Si}$ & -4.41392700 & -1.70960600 & -1.54117000 \\
$\mathrm{Si}$ & 3.66446900 & -2.82925100 & -1.62084400 \\
$\mathrm{Si}$ & 2.56959500 & 2.89094400 & 1.76516900 \\
$\mathrm{C}$ & -3.93214300 & -0.67406200 & -0.06560000 \\
$\mathrm{C}$ & -3.94392300 & 0.75344700 & 0.02147300 \\
$\mathrm{C}$ & -3.57201200 & 1.14557000 & 1.33283300 \\
$\mathrm{C}$ & -3.32140700 & -0.02925100 & 2.08547700 \\
$\mathrm{C}$ & -3.53977800 & -1.14144200 & 1.23231200 \\
$\mathrm{C}$ & 2.70666900 & -2.64077200 & -0.02549400 \\
& \multicolumn{3}{c}{$\mathrm{S} 156$} \\
\end{tabular}




\begin{tabular}{|c|c|c|c|}
\hline $\mathrm{C}$ & 2.93727100 & -1.65777900 & 0.99356000 \\
\hline $\mathrm{C}$ & 2.02973200 & -1.87556800 & 2.05889000 \\
\hline $\mathrm{C}$ & 1.22530700 & -2.99592200 & 1.72721200 \\
\hline $\mathrm{C}$ & 1.63310400 & -3.46593700 & 0.45453300 \\
\hline $\mathrm{C}$ & 1.67854600 & 3.05364000 & 0.13374600 \\
\hline $\mathrm{C}$ & 0.39525800 & 3.64278100 & -0.07859600 \\
\hline $\mathrm{C}$ & 0.10173000 & 3.62347100 & -1.46325700 \\
\hline $\mathrm{C}$ & 1.21410900 & 3.05025200 & -2.14190600 \\
\hline $\mathrm{C}$ & 2.17610800 & 2.70086400 & -1.16415900 \\
\hline $\mathrm{H}$ & -0.94290000 & -1.67997000 & 0.54824700 \\
\hline $\mathrm{H}$ & 0.25302800 & 0.23028100 & 0.92251000 \\
\hline $\mathrm{H}$ & -0.39787400 & -0.41478800 & -1.02075600 \\
\hline $\mathrm{H}$ & -0.95059700 & 1.65912800 & 0.57180100 \\
\hline $\mathrm{H}$ & 1.61830700 & 0.07582900 & -0.83615400 \\
\hline $\mathrm{H}$ & -0.81883500 & 3.95853300 & -1.92420200 \\
\hline $\mathrm{H}$ & -0.26352200 & 4.01044200 & 0.69738500 \\
\hline $\mathrm{H}$ & 1.30686000 & 2.91190800 & -3.20948300 \\
\hline $\mathrm{H}$ & 3.12444200 & 2.21730600 & -1.36282500 \\
\hline $\mathrm{H}$ & 3.48286200 & 1.71695700 & 1.74401600 \\
\hline $\mathrm{H}$ & 3.39833500 & 4.09829900 & 2.04132700 \\
\hline $\mathrm{H}$ & 1.59262700 & 2.74313600 & 2.87550700 \\
\hline $\mathrm{H}$ & -3.46597500 & 2.16345000 & 1.68605500 \\
\hline $\mathrm{H}$ & -4.17008600 & 1.42985900 & -0.79350200 \\
\hline $\mathrm{H}$ & -2.99558700 & -0.06872500 & 3.11819600 \\
\hline $\mathrm{H}$ & -3.41335400 & -2.18031100 & 1.51223900 \\
\hline $\mathrm{H}$ & -5.86388600 & -2.05539200 & -1.50719400 \\
\hline $\mathrm{H}$ & -4.14979300 & -0.96105300 & -2.79718400 \\
\hline $\mathrm{H}$ & -3.64827300 & -2.98499200 & -1.54332400 \\
\hline $\mathrm{H}$ & 2.73677300 & -3.08818700 & -2.75529000 \\
\hline $\mathrm{H}$ & 4.43785400 & -1.58802700 & -1.87675200 \\
\hline $\mathrm{H}$ & 4.61087100 & -3.97597100 & -1.53876400 \\
\hline $\mathrm{H}$ & 3.66241300 & -0.85559000 & 0.94000800 \\
\hline $\mathrm{H}$ & 1.93838900 & -1.26837100 & 2.95084000 \\
\hline $\mathrm{H}$ & 0.41290800 & -3.39451300 & 2.32116200 \\
\hline $\mathrm{H}$ & 1.19290500 & -4.30438500 & -0.07458400 \\
\hline $\mathrm{H}$ & -1.49674000 & 1.12489600 & -1.20262000 \\
\hline $\mathrm{H}$ & 0.11671400 & 0.96756500 & -2.38406500 \\
\hline
\end{tabular}

\begin{tabular}{lccc}
$\mathbf{T S 1}_{\mathbf{F}} \mathbf{A F}^{\mathbf{A F}}$ & & & \\
$\mathrm{Ti}$ & 0.78504800 & -1.35180000 & 0.07646100 \\
$\mathrm{Ti}$ & -1.70499100 & -0.00775700 & 0.37199200 \\
$\mathrm{Ti}$ & 0.31289600 & 1.36195600 & -0.75013800 \\
$\mathrm{Si}$ & -4.40880400 & -1.63015100 & -1.59506100 \\
$\mathrm{Si}$ & 3.65054000 & -2.83488300 & -1.62253500 \\
$\mathrm{Si}$ & 2.53732000 & 2.96856600 & 1.78917900 \\
$\mathrm{C}$ & -3.93647300 & -0.63906500 & -0.08567300 \\
\multicolumn{3}{c}{$\mathrm{S} 157$} \\
\hline
\end{tabular}




\begin{tabular}{|c|c|c|c|}
\hline $\mathrm{C}$ & -3.91587300 & 0.78473400 & 0.03513900 \\
\hline $\mathrm{C}$ & -3.56904700 & 1.13688200 & 1.36545100 \\
\hline $\mathrm{C}$ & -3.36407400 & -0.05996900 & 2.09498000 \\
\hline $\mathrm{C}$ & -3.58433500 & -1.14701700 & 1.20884000 \\
\hline $\mathrm{C}$ & 2.69990600 & -2.65557800 & -0.02231600 \\
\hline $\mathrm{C}$ & 2.92212100 & -1.66419000 & 0.99056300 \\
\hline $\mathrm{C}$ & 2.02225600 & -1.88879800 & 2.06068700 \\
\hline $\mathrm{C}$ & 1.23118900 & -3.02121700 & 1.73839600 \\
\hline $\mathrm{C}$ & 1.63959800 & -3.49247900 & 0.46650400 \\
\hline $\mathrm{C}$ & 1.69231100 & 3.07953000 & 0.13123000 \\
\hline $\mathrm{C}$ & 0.42468500 & 3.67645500 & -0.13272200 \\
\hline $\mathrm{C}$ & 0.15798100 & 3.60646400 & -1.51924100 \\
\hline $\mathrm{C}$ & 1.27480700 & 2.98056400 & -2.15448100 \\
\hline $\mathrm{C}$ & 2.20968000 & 2.65858300 & -1.14042200 \\
\hline $\mathrm{H}$ & -0.95781600 & -1.71529700 & 0.60532200 \\
\hline $\mathrm{H}$ & 0.20972800 & 0.19617300 & 0.93356500 \\
\hline $\mathrm{H}$ & -0.44365100 & -0.46136200 & -0.99607100 \\
\hline $\mathrm{H}$ & -1.04493500 & 1.67289600 & 0.43952700 \\
\hline $\mathrm{H}$ & 1.59507500 & -0.01534500 & -0.88099200 \\
\hline $\mathrm{H}$ & -0.74344300 & 3.94916200 & -2.01123200 \\
\hline $\mathrm{H}$ & -0.24846900 & 4.07414500 & 0.61615300 \\
\hline $\mathrm{H}$ & 1.39276500 & 2.80539300 & -3.21486300 \\
\hline $\mathrm{H}$ & 3.15521300 & 2.15409900 & -1.29740200 \\
\hline $\mathrm{H}$ & 3.38688100 & 1.74877300 & 1.86748400 \\
\hline $\mathrm{H}$ & 3.42661700 & 4.14223400 & 2.02080600 \\
\hline $\mathrm{H}$ & 1.52755400 & 2.93821600 & 2.88010500 \\
\hline $\mathrm{H}$ & -3.44647400 & 2.14361100 & 1.74407800 \\
\hline $\mathrm{H}$ & -4.09971900 & 1.48703300 & -0.76920200 \\
\hline $\mathrm{H}$ & -3.06814400 & -0.13192800 & 3.13479000 \\
\hline $\mathrm{H}$ & -3.49014000 & -2.19508700 & 1.46611600 \\
\hline $\mathrm{H}$ & -5.87194000 & -1.91299000 & -1.61856600 \\
\hline $\mathrm{H}$ & -4.06760100 & -0.87310700 & -2.82800400 \\
\hline $\mathrm{H}$ & -3.69488700 & -2.93496400 & -1.59312300 \\
\hline $\mathrm{H}$ & 2.71975500 & -3.09166700 & -2.75536400 \\
\hline $\mathrm{H}$ & 4.42111000 & -1.59184400 & -1.87714000 \\
\hline $\mathrm{H}$ & 4.59894400 & -3.98091500 & -1.55032700 \\
\hline $\mathrm{H}$ & 3.63647400 & -0.85302300 & 0.92973900 \\
\hline $\mathrm{H}$ & 1.92576500 & -1.27743700 & 2.94926100 \\
\hline $\mathrm{H}$ & 0.42696100 & -3.42748800 & 2.33820100 \\
\hline $\mathrm{H}$ & 1.20890500 & -4.34016000 & -0.05577300 \\
\hline $\mathrm{H}$ & -1.14053700 & 1.18226900 & -1.72892300 \\
\hline $\mathrm{H}$ & -0.29415100 & 1.07696900 & -2.33488400 \\
\hline
\end{tabular}

\begin{tabular}{cccc}
$\mathbf{A 2} \mathbf{2}_{\mathbf{F}} \mathbf{A F}$ & & \\
$\mathrm{Ti}$ & 1.06557800 & -1.12859400 & 0.11682700 \\
$\mathrm{Ti}$ & -1.63518300 & -0.24946000 & 0.17139800 \\
\multicolumn{3}{c}{$\mathrm{S} 158$}
\end{tabular}




\begin{tabular}{|c|c|c|c|}
\hline $\mathrm{Ti}$ & 0.40101400 & 1.32893500 & -1.05951100 \\
\hline $\mathrm{Si}$ & -4.32583300 & -2.15283800 & -1.62713400 \\
\hline $\mathrm{Si}$ & 4.05322800 & -2.64802300 & -1.35984300 \\
\hline $\mathrm{Si}$ & 0.66109600 & 3.27668400 & 2.13863800 \\
\hline $\mathrm{C}$ & -3.84881900 & -1.13244600 & -0.14708100 \\
\hline $\mathrm{C}$ & -3.94022000 & 0.28829300 & -0.01477100 \\
\hline $\mathrm{C}$ & -3.51849500 & 0.66739700 & 1.28777700 \\
\hline $\mathrm{C}$ & -3.15414100 & -0.51703800 & 1.98494700 \\
\hline $\mathrm{C}$ & -3.35379100 & -1.61237800 & 1.10622600 \\
\hline $\mathrm{C}$ & 3.07287000 & -2.29910700 & 0.19109900 \\
\hline $\mathrm{C}$ & 3.21250100 & -1.15622200 & 1.04907100 \\
\hline $\mathrm{C}$ & 2.32816400 & -1.29191000 & 2.14806800 \\
\hline $\mathrm{C}$ & 1.62549900 & -2.51265900 & 1.99724300 \\
\hline $\mathrm{C}$ & 2.07547200 & -3.12862100 & 0.80221100 \\
\hline $\mathrm{C}$ & 0.86467500 & 3.24631400 & 0.28023700 \\
\hline $\mathrm{C}$ & -0.10323200 & 3.64221300 & -0.69116700 \\
\hline $\mathrm{C}$ & 0.46570300 & 3.52642100 & -1.98096700 \\
\hline $\mathrm{C}$ & 1.80244500 & 3.05705500 & -1.83279800 \\
\hline $\mathrm{C}$ & 2.04473300 & 2.88906300 & -0.44628900 \\
\hline $\mathrm{H}$ & -0.55400400 & -1.76803000 & 0.55121400 \\
\hline $\mathrm{H}$ & 0.21167000 & 0.50753100 & 0.67075400 \\
\hline $\mathrm{H}$ & -0.18082400 & -0.52069600 & -1.21107100 \\
\hline $\mathrm{H}$ & -1.38792900 & 1.27341300 & -0.96042200 \\
\hline $\mathrm{H}$ & 1.79957100 & 0.11403200 & -1.06875000 \\
\hline $\mathrm{H}$ & -0.03844200 & 3.73488300 & -2.91720000 \\
\hline $\mathrm{H}$ & -1.12075900 & 3.94454000 & -0.47934300 \\
\hline $\mathrm{H}$ & 2.50626700 & 2.85854100 & -2.63185300 \\
\hline $\mathrm{H}$ & 2.96593700 & 2.52386400 & -0.00983800 \\
\hline $\mathrm{H}$ & 1.57987300 & 2.29330000 & 2.76901000 \\
\hline $\mathrm{H}$ & 1.00592200 & 4.62542000 & 2.67156000 \\
\hline $\mathrm{H}$ & -0.74302700 & 2.97875400 & 2.51748900 \\
\hline $\mathrm{H}$ & -3.47745400 & 1.67743300 & 1.67739700 \\
\hline $\mathrm{H}$ & -4.25937900 & 0.97109900 & -0.79383800 \\
\hline $\mathrm{H}$ & -2.78297400 & -0.57322700 & 3.00130900 \\
\hline $\mathrm{H}$ & -3.13691900 & -2.64876100 & 1.33799000 \\
\hline $\mathrm{H}$ & -5.75899700 & -2.56777100 & -1.58859300 \\
\hline $\mathrm{H}$ & -4.12621300 & -1.37338300 & -2.88031600 \\
\hline $\mathrm{H}$ & -3.50708700 & -3.39475900 & -1.68737500 \\
\hline $\mathrm{H}$ & 3.17062000 & -3.20597900 & -2.42069400 \\
\hline $\mathrm{H}$ & 4.67853700 & -1.38977700 & -1.84227500 \\
\hline $\mathrm{H}$ & 5.13168700 & -3.64232500 & -1.10151500 \\
\hline $\mathrm{H}$ & 3.87204700 & -0.31619700 & 0.86994600 \\
\hline $\mathrm{H}$ & 2.18011800 & -0.56663600 & 2.93880300 \\
\hline $\mathrm{H}$ & 0.84797600 & -2.88585400 & 2.65053200 \\
\hline $\mathrm{H}$ & 1.70165600 & -4.06590900 & 0.40422500 \\
\hline $\mathrm{H}$ & -0.25265000 & 0.95575000 & -2.96490700 \\
\hline
\end{tabular}


